\author{
UNIVERSIDADE DE SÃO PAULO \\ ESCOLA DE ENGENHARIA DE SÃO CARLOS \\ ÁREA DE PÓS-GRADUAÇÃO EM ENGENHARIA MECÂNICA
}

\title{
CORREÇÃO DE EFEITOS VISCOSOS NA SOLUÇÃO DO ESCOAMENTO POTENCIAL DE PEQUENAS PERTURBAÇÕES EM REGIME TRANSÔNICO NO DOMÍNIO DA FREQÜÊNCIA
}

Lee Yun Sheng

Orientador: Prof. Dr. Paulo Celso Greco Jr.

Texto redigido para o Exame de Defesa apresentado à Escola de Engenharia, de São Carlos, Universidade de São Paulo, como parte dos requisitos para obtenção do título de Doutor em Engenharia Mecânica.

São Carlos 2007 

Aos

Meus

PAIS 



\section{Agradecimentos}

Ao professor Paulo Greco, pela orientação, apoio, paciência e principalmente pelo estímulo constante transmitido durante toda a pesquisa.

Aos meus amigos e colegas de sala pelos momentos de descontração e apoio.

À Coordenação de Aperfeiçoamento de Pessoal de Nível Superior (CAPES) pela concessão de bolsa para a elaboração deste trabalho.

E para finalizar, a todos que contribuíram direta e indiretamente a elaboração deste trabalho. 

Nunca se deve engatinhar quando o impulso é voar. (Helen Keller) 



\section{RESUMO}

\section{LEE, Y. S. Correção de efeitos viscosos na solução do escoamento potencial de peque-}

nas perturbações em regime transônico no domínio da freqüência. 2007, 95 f. Tese (Doutorado) - Escola de Engenharia de São Carlos, Universidade de São Paulo, São Paulo, 2007.

Um método de correção viscosa é aplicado na solução da equação potencial transônica de pequenas perturbações (TSD) no domínio da frequência. O objetivo é melhorar os resultados transônicos em que a interação choque/camada-limite é importante. A espessura de deslocamento da camada limite é estimada, a partir dos resultados da análise do escoamento invíscido, usando um método integral. A espessura de deslocamento é usada, então, para modificar a geometria das superfícies de sustentação e um novo resultado invíscido é obtido. Esse processo é repetido até que se atinja a convergência. No passado esse método foi aplicado, com bons resultados, na análise no domínio do tempo. No domínio da frequência os termos espaciais não lineares são preservados usando uma técnica de transformação conhecida como média harmônica. A principal razão para usar equação TSD ainda é o custo computacional, especialmente em se tratando de configurações completas de aeronaves. Um código de computador original é desenvolvido para análise bidimensional e um código de computador tridimensional existente é modificado para incluir a correção viscosa. A equação TSD é aproximada usando o método das diferenças finitas e resolvida usando sobrerelaxação sucessiva por linhas. Nos dois códigos é utilizada correção para vorticidade e variação de entropia. Os resultados têm boa correlação com dados experimentais publicados para a distribuição de pressão em regime transônico estacionário.

Palavras-chaves: Equações de pequenas perturbações. Transônico. Dinâmica dos fluídos computacional. 



\section{Abstract}

\section{LEE, Y. S. Correção de efeitos viscosos na solução do escoamento potencial de peque-}

nas perturbações em regime transônico no domínio da freqüiência. 2007, 95 f. Tese (Doutorado) - Escola de Engenharia de São Carlos, Universidade de São Paulo, São Paulo, 2007 .

A viscous correction method is applied to the solution of the transonic small disturbance (TSD) potential equation in the frequency domain. The objective is to improve transonic results for which shock/boundary-layer interaction is important. Boundary-layer displacement thickness is calculated, with an integral method, using the results from an inviscid flow analysis. The calculated displacement thickness is then used to modify the lifting surface geometry and a new inviscid result is obtained. This process is repeated until convergence is achieved. In the past that method has been applied to time domain analysis with good results. In frequency domain the spatial nonlinear terms are preserved using a transformation technique known as harmonic averaging. The main reason for using the TSD equation still is computational cost, especially when dealing with complete aircraft configurations. An original computer code is developed for two-dimensional analysis and an existing threedimensional computer code is extended to include the viscous correction. The transonic small disturbance potential equation is approximated using the finite difference method and solved through successive line over-relaxation. Both codes include correction for vorticity and variation in entropy. Results for several airfoil sections are obtained. The results compare well with published experimental data for steady transonic pressure distribution.

Keywords: Transonic small disturbance (TSD). Potential equation. Computational fluid dynamics. 



\title{
Lista de Símbolos
}

\author{
Símbolo Descrição \\ $\alpha \quad$ Ângulo de ataque \\ $\alpha^{*} \quad$ Ângulo de ataque num determinado instante de tempo \\ $\bar{v} \quad$ Viscosidade cinemática \\ $\delta \quad$ Ângulo diferença de fase (atraso) entre o movimento e a resposta \\ aerodinâmica \\ $\delta^{*} \quad$ Espessura de deslocamento \\ $\hat{\delta} \quad$ Espessura de deslocamento nas coordenadas transformadas \\ $\gamma \quad$ Razão do calor específico \\ $\omega \quad$ Frequência de oscilação \\ $\Phi \quad$ Potencial de velocidade total \\ $\phi \quad$ Potencial de velocidade de perturbação \\ $\rho \quad$ Densidade \\ $\tau \quad$ Tensão de cisalhamento \\ $\theta \quad$ Ângulo de fase \\ $\hat{\theta} \quad$ Função da espessura de momentum nas coordenadas transformadas
}




\section{Símbolo Descrição}

$a$

Velocidade do som local

$a_{\infty} \quad$ Velocidade do som do fluxo livre

$b_{0} \quad$ Comprimento de referência (em geral é o comprimento de um quarto de corda da asa ou de um quarto de corda aerodinâmica)

$C_{f} \quad$ Coeficiente de atrito

$C_{p} \quad$ Coeficiente de pressão

f $\quad$ função da localização da superfície do perfil

$f^{s} \quad$ Forma da superfície aerodinâmica

$f^{v} \quad$ Deslocamento da superfície aerodinâmica em oscilação

$\hat{H}_{i} \quad$ Fator de forma incompressível transformado

M Número de Mach

$M_{\infty} \quad$ Número de Mach do fluxo livre

$n \quad$ Direção normal ao escoamento

p Pressão

$\operatorname{Pr} \quad$ Número de Prandalt

$s \quad$ Direção do escoamento

$\frac{s}{c_{v}} \quad$ Coeficiente de salto de entropia, calculado usando a relação de Rankine-

Hugoniot, necessária para os cálculos de correção de vorticidade e de entropia

$t \quad$ Variável tempo

Temperatura

$U \quad$ Velocidade na direção $x$

$u \quad$ Componente de velocidade média na direção do escoamento

$V \quad$ Velocidade na direção $y$

$V_{\infty} \quad$ Velocidade do fluxo livre

W Velocidade na direção $z$

w Componente de velocidade média na direção normal ao escoamento

$x, y, z \quad$ Coordenadas global

$X, Y, Z \quad$ Coordenadas local

$x_{p} \quad$ Posição do centro de arfagem 


\section{Siglas}

$A D I$

$C F D$

EE

$F P E$

$T S D$

$U s T S D$

FNS

$T L N S$

RANS

$T S D+E n t+$ Vort

FLO52

\section{Descrição}

Algoritmo implícito de diferenças finitas de direção alternada (Alternating-Direction Implicit)

Dinâmica dos Fluidos Computacional

(Computational Fluid Dynamics)

Equações de Euler

Equação potencial completa (Full Potential Equation)

Pequenas perturbações transônica

(Transonic Small Disturbance)

Programa de pequenas perturbações transônica não

estacionário (Unsteady Transonic Small Disturbance program)

Equações de Navier-Stokes completa (Full Navier-Stokes)

Camada limite fina (Thin Layer Navier-Stokes)

Média de Reynolds (Reynolds Averaged Navier-Stokes)

Programa de pequenas perturbações transônica estacionário

desenvolvida durante o doutorado com correção de entropia

e vorticidade.

$T S D+E n t+V o r t+C L$ Programa de pequenas perturbações transônica estacionário desenvolvida durante o doutorado com correção de entropia e vorticidade, mais correção viscosa.

"Programa de pequenas perturbações transônica estacionário

utilizando as equações TSD na forma conservativa ${ }^{[5]}$." 


$\begin{array}{ll}\text { Subscrito } & \text { Descrição } \\ 0 & \text { Condição de estagnação } \\ e & \text { Condição na fronteira da camada limite } \\ t & \text { Diferenciação em } t \\ w & \text { Condição na parede } \\ x & \text { Diferenciação em } x \\ y & \text { Diferenciação em } y \\ z & \text { Diferenciação em } z\end{array}$

\section{Sobrescrito Descrição}

$S$

Estacionário

$u$

Não estacionário 


\section{Lista de Figuras}

2.1 Definição dos eixos $x, y$ e $z$ em torno de um perfil. . . . . . . . . . . 10

2.2 Sistema de coordenadas usado para definir a geometria das superfícies de sustentação. . . . . . . . . . . . . . . . . . . . . . 17

2.3 Definição das fronteiras externas do domínio computacional. . . . . . . . . 21

2.4 Definições para a malha computacional. . . . . . . . . . . . . . . 32

2.5 Exemplo de malha utilizada nas simulações (esta é uma visão ampliada em

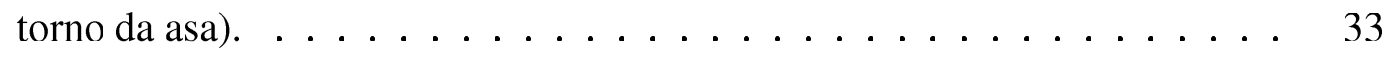

2.6 Condições de contorno na malha. . . . . . . . . . . . . . . 34

2.7 Distribuição do coeficiente de pressão para o perfil NACA0012 em $M_{\infty}=$ 0,750 e $\alpha=1,00^{\circ}$, com os segintes refinamentos na corda: 35 pontos, 39 pontos e 41 pontos . . . . . . . . . . . . . . . .

2.8 Distribuição do coeficiente de pressão para o perfil NACA0012 em $M_{\infty}=$ 0,750 e $\alpha=1,00^{\circ}$, com os segintes refinamentos na corda: 41 pontos, 45 pontos e 51 pontos . . . . . . . . . . . . . . . .

2.9 Distribuição do coeficiente de pressão para o perfil NACA0012 em $M_{\infty}=$ 0,800 e $\alpha=1,25^{\circ}$, com os segintes refinamentos na corda: 35 pontos, 39 pontos e 41 pontos . . . . . . . . . . . . . . . . . 36

2.10 Distribuição do coeficiente de pressão para o perfil NACA0012 em $M_{\infty}=$ 0,800 e $\alpha=1,25^{\circ}$, com os segintes refinamentos na corda: 41 pontos, 45 pontos e 51 pontos . . . . . . . . . . . . . . .

2.11 Distribuição do coeficiente de pressão para o perfil NACA0012 em $M_{\infty}=$ 0,750 e $\alpha=1,00^{\circ}$, com a distância da frontria superior e infreior, $n h$, variando em 1,2 e 3 cordas. . . . . . . . . . . . . . . . 
2.12 Distribuição do coeficiente de pressão para o perfil NACA0012 em $M_{\infty}=$ 0,750 e $\alpha=1,00^{\circ}$, com a distância da frontria superior e infreior, $n h$, variando em 3,5 e 7 cordas. . . . . . . . . . . . . . . . . . .

2.13 Distribuição do coeficiente de pressão para o perfil NACA0012 em $M_{\infty}=$ 0,750 e $\alpha=1,00^{\circ}$, com a distância antes do bordo de ataque e pós bordo de fuga, $n a$ e $n d$, variando em 1,2 e 5 cordas. . . . . . . . . . . . .

3.1 Seção do perfil RAE2822. . . . . . . . . . . . . . . . . . . . . . . . . 40

3.2 Seção do perfil NACA0012. . . . . . . . . . . . . . . . . . . . . 41

3.3 Seção do perfil NLR7301. . . . . . . . . . . . . . . . . . . . . . . . 41

3.4 Gráfico do resíduo usando TSD + Ent + Vort + CL para seção do perfil RAE2822 com $M_{\infty}=0,729$ e $\alpha=2,31^{\circ} \ldots \ldots \ldots \ldots$

3.5 Comportamento da distribuição de pressão ao longo das iterações na simulação no perfil RAE2822 com $M_{\infty}=0,729$ e $\alpha=2,31^{\circ}$, usando TSD + Ent + Vort + CL. . . . . . . . . . . . . . . . . . . . .

3.6 Gráfico do resíduo usando TSD+Ent+Vort+CL para seção do perfil RAE2822 com $M_{\infty}=0,730$ e $\alpha=3,19^{\circ}$.

3.7 Comportamento da distribuição de pressão ao longo das iterações na simulação no perfil RAE2822 com $M_{\infty}=0,730$ e $\alpha=3,19^{\circ}$, usando TSD + Ent + Vort + CL . . . . . . . . . . . . . . . . . . . . . . .

3.8 Distribuição do coeficiente de pressão para a seção do perfil RAE2822 com $M_{\infty}=0,730$ e $\alpha=3,19^{\circ} \ldots \ldots \ldots \ldots \ldots$

3.9 Distribuição do coeficiente de pressão para a seção do perfil RAE2822 com $M_{\infty}=0,729$ e $\alpha=2,31^{\circ} \ldots \ldots \ldots \ldots$

3.10 Espessura de deslocamento para a seção do perfil RAE2822 $\mathrm{com} M_{\infty}=$

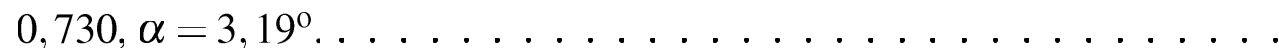

3.11 Espessura de deslocamento para a seção do perfil RAE2822 $\mathrm{com} M_{\infty}=$

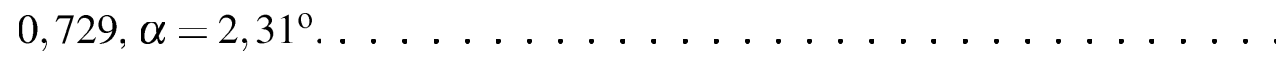

3.12 Comparação da espessura de deslocamento para a seção do perfil RAE2822 $\operatorname{com} M_{\infty}=0,730, \alpha=3,19^{\circ}$ e $\operatorname{com} M_{\infty}=0,729, \alpha=2,31^{\circ} \ldots \ldots \ldots$

3.13 Correção da seção do perfil RAE2822 com $M_{\infty}=0,730, \alpha=3,19^{\circ}$, usando espessura de deslocamento. 
3.14 Correção da seção do perfil RAE2822 com $M_{\infty}=0,729, \alpha=2,31^{\circ}$, usando espessura de deslocamento. . . . . . . . . . . . . . . . . . . .

3.15 Gráfico do campo de velocidade em torno do perfil RAE2822 $\mathrm{com} M_{\infty}=$ $0,730, \alpha=3,19^{\circ}$, usando TSD+Ent+Vort+CL.

3.16 Gráfico do campo de velocidade em torno do perfil RAE2822 com $M_{\infty}=$ $0,729, \alpha=2,31^{\circ}$, usando TSD+Ent+Vort+CL. . . . . . . . . . . 50

3.17 Gráfico do potencial em torno do perfil RAE2822 $\operatorname{com} M_{\infty}=0,730, \alpha=$ $3,19^{\circ}$, usando TSD+Ent+Vort+CL. . . . . . . . . . . . . . . .

3.18 Gráfico do potencial em torno do perfil RAE2822 $\operatorname{com} M_{\infty}=0,729, \alpha=$ $2,31^{\circ}$, usando TSD+Ent+Vort+CL . . . . . . . . . . . . . . . .

3.19 Gráfico do número de Mach em torno do perfil RAE2822 com $M_{\infty}=0,730$, $\alpha=3,19^{\circ}$, usando TSD+Ent+Vort+CL. . . . . . . . . . . . . . . .

3.20 Gráfico do número de Mach em torno do perfil RAE2822 com $M_{\infty}=0,729$, $\alpha=2,31^{\circ}$, usando TSD+Ent+Vort+CL

3.21 Coeficiente de pressão para a seção do perfil NACA0012 $\operatorname{com} M_{\infty}=0,750$, $\alpha=1,00^{\circ}$.

3.22 Coeficiente de pressão para a seção do perfil NACA0012 com $M_{\infty}=0,750$, $\alpha=3,00^{\circ}$.

3.23 Coeficiente de pressão para a seção do perfil NACA0012 $\operatorname{com} M_{\infty}=0,800$, $\alpha=1,25^{\circ}$.

3.24 Espessura de deslocamento para a seção NACA0012 $\operatorname{com} M_{\infty}=0,750, \alpha=$ $1,00^{\circ}$, usando TSD+Ent+Vort+CL

3.25 Espessura de deslocamento para a seção NACA0012 $\operatorname{com} M_{\infty}=0,750, \alpha=$ $1,00^{\circ}$, usando TSD+Ent+Vort+CL . . . . . . . . . . . . . . . . 56

3.26 Espessura de deslocamento para a seção NACA0012 $\operatorname{com} M_{\infty}=0,800, \alpha=$ $1,25^{\circ}$, usando TSD+Ent+Vort+CL . . . . . . . . . . . . . . .

3.27 Comparação da espessura de deslocamento para a seção do perfil NACA0012 $\operatorname{com} M_{\infty}=0,750, \operatorname{com} \alpha=1,00^{\circ}$ e $\alpha=3,00^{\circ} \ldots \ldots$

3.28 Correção da seção do perfil NACA0012 com $M_{\infty}=0,750, \alpha=1,00^{\circ}$, usando espessura de deslocamento. 
3.29 Correção da seção do perfil NACA0012 $\operatorname{com} M_{\infty}=0,750, \alpha=3,00^{\circ}$, usando espessura de deslocamento. . . . . . . . . . . . . . . . . 58

3.30 Correção da seção do perfil NACA0012 com $M_{\infty}=0,800, \alpha=1,25^{\circ}$, usando espessura de deslocamento. . . . . . . . . . . . . . . . . 58

3.31 Gráfico do número de Mach em torno do perfil NACA0012 $\mathrm{com} M_{\infty}=$ $0,750, \alpha=1,00^{\circ}$, usando TSD+Ent+Vort+CL. . . . . . . . . . . . . 59

3.32 Gráfico do número de Mach em torno do perfil NACA0012 $\mathrm{com} M_{\infty}=$ $0,750, \alpha=3,00^{\circ}$, usando TSD+Ent+Vort+CL. . . . . . . . . .

3.33 Gráfico do número de Mach em torno do perfil NACA0012 com $M_{\infty}=$ $0,800, \alpha=1,25^{\circ}$, usando TSD+Ent+Vort+CL . . . . . . . . . . 60

3.34 Coeficiente de pressão para a seção do perfil NLR7301 $\operatorname{com} M_{\infty}=0,752$, $\alpha=0,37^{\circ}$.

3.35 Coeficiente de pressão para a seção do perfil NLR7301 $\operatorname{com} M_{\infty}=0,753$, $\alpha=-0,08^{\circ}$.

3.36 Espessura de deslocamento para a seção do perfil NLR7301 com $M_{\infty}=$ $0,752, \alpha=0,37^{\circ}$. usando TSD+Ent+Vort+CL. . . . . . . . . . .

3.37 Espessura de deslocamento para a seção NLR7301 $\operatorname{com} M_{\infty}=0,753, \alpha=$ $-0,08^{\mathrm{o}}$, usando TSD+Ent+Vort+CL. . . . . . . . . . . . .

3.38 Gráfico do número de Mach em torno do perfil NLR7301 com $M_{\infty}=0,752$, $\alpha=0,37^{\circ}$, usando TSD+Ent+Vort+CL. . . . . . . . . . . . . . .

3.39 Gráfico do número de Mach em torno do perfil NLR7301 $\operatorname{com} M_{\infty}=0,753$, $\alpha=-0,08^{\circ}$, usando TSD+Ent+Vort+CL

4.1 Resíduo do programa UsTSD aplicado na seção RAE2822 com $M_{\infty}=0,730$, $\alpha=3,19^{\circ}$, sem e com Correção Viscosa. . . . . . . . . . . . . . . 66

4.2 Resíduo do programa UsTSD aplicado na seção RAE2822 $\operatorname{com} M_{\infty}=0,730$, $\alpha=3,1^{\circ}$, sem e com Correção Viscosa.

4.3 Comparação da espessura de deslocamento para a seção RAE2822 com $M_{\infty}=0,730, \alpha=3,19^{\circ}$ e com $M_{\infty}=0,729, \alpha=2,31^{\circ}$, usando UsTSD. 
4.4 Comparação da espessura de deslocamento para a seção do perfil RAE2822 com $M_{\infty}=0,730, \alpha=3,19^{\circ}$ e com $M_{\infty}=0,729, \alpha=2,31^{\circ}$, usando o programa TSD. (Corresponde a Figura 3.12) . . . . . . . . . . . . . . .

4.5 Distribuição do coeficiente de pressão para a seção RAE2822 $\mathrm{com} M_{\infty}=$ 0,730, $\alpha=3,19^{\circ}$, usando UsTSD sem e com Correção Viscosa. . . . . . .

4.6 Distribuição do coeficiente de pressão para a seção RAE2822 com $M_{\infty}=$ 0,729, $\alpha=2,31^{\circ}$, usando UsTSD sem e com Correção Viscosa. . . . . . .

4.7 Distribuição do coeficiente de pressão para a seção do perfil RAE2822 com $M_{\infty}=0,730$ e $\alpha=3,19^{\circ}$, usando TSD sem e com Correção Viscosa.(Corresponde a Figura 3.8).

4.8 Distribuição do coeficiente de pressão para a seção do perfil RAE2822 com $M_{\infty}=0,729$ e $\alpha=2,31^{\circ}$, usando TSD sem e com Correção Viscosa.(Corresponde a Figura 3.9).

4.9 Distribuição do coeficiente de pressão para a seção NACA0012 com $M_{\infty}=$ $0,750, \alpha=1,00^{\circ}$, usando UsTSD sem e com Correção Viscosa. . . . . . .

4.10 Distribuição do coeficiente de pressão para a seção NACA0012 com $M_{\infty}=$ 0,750, $\alpha=3,00^{\circ}$, usando UsTSD sem e com Correção Viscosa. . . . . . .

4.11 Comparação da espessura de deslocamento para a seção NACA0012 com $M_{\infty}=0,750, \alpha=1,00^{\circ}$ e com $M_{\infty}=0,750, \alpha=3,00^{\circ}$, usando UsTSD com Correção Viscosa. . . . . . . . . . . . . . . . . . . . .

4.12 Distribuição do coeficiente de pressão para a seção NACA0012 com $M_{\infty}=$ 0,800, $\alpha=1,25^{\circ}$, usando UsTSD sem e com Correção Viscosa e do programa FLO52 $[5] \ldots \ldots \ldots \ldots \ldots \ldots$

4.13 Comparação da espessura de deslocamento para a seção NRL7301 com $M_{\infty}=0,752, \alpha=0,37^{\circ}$, usando UsTSD sem e com Correção Viscosa. . 76

4.14 Comparação da espessura de deslocamento para a seção NRL7301 com $M_{\infty}=0,753, \alpha=-0,08^{\circ}$, usando UsTSD sem e com Correção Viscosa.

4.15 Distribuição do coeficiente de pressão para a seção NRL7301 com $M_{\infty}=$ $0,752, \alpha=0,37^{\circ}$, usando UsTSD sem e com Correção Viscosa. . . . . . .

4.16 Distribuição do coeficiente de pressão para a seção NRL7301 com $M_{\infty}=$ 0,753, $\alpha=-0,08^{\circ}$, usando UsTSD sem e com Correção Viscosa. . . . . . 
4.17 Coeficiente de pressão para a seção do perfil NLR7301 com $M_{\infty}=0,752$, $\alpha=0,37^{\circ}$, usando TSD sem e com Correção Viscosa. (Corresponde a Figura 3.34) . . . . . . . . . . . . . . . . .

4.18 Coeficiente de pressão para a seção do perfil NLR7301 $\operatorname{com} M_{\infty}=0,753$, $\alpha=-0,08^{\circ}$, usando TSD sem e com Correção Viscosa. (Corresponde a Figura 3.35$) \ldots \ldots \ldots 7 . \ldots \ldots$

4.19 NRL F5 com a posição dos orifícios de tomada de pressão e dos transdutores. 82

4.20 NRL F5 em Mach 0,597, $\alpha=0,493^{\circ}$ com $R e=4,77 \times 10^{6}$ (Parte I) . . . . 83

4.21 NRL F5 em Mach 0,597, $\alpha=0,493^{\circ} \operatorname{com} R e=4,77 \times 10^{6}$ (Parte II) . . . . 84

4.22 NRL F5 em Mach 0,896, $\alpha=0,497^{\circ} \operatorname{com} R e=5,79 \times 10^{6}$ (Parte I). . . . . 85

4.23 NRL F5 em Mach 0,896, $\alpha=0,497^{\circ} \mathrm{com} R e=5,79 \times 10^{6}$ (Parte II). . . . . 86

4.24 NRL F5 em Mach 0,946, $\alpha=-0,004^{\circ}$ com $R e=5,89 \times 10^{6}$ (Parte I). . . . 87

4.25 NRL F5 em Mach 0,946, $\alpha=-0,004^{\circ}$ com $R e=5,89 \times 10^{6}$ (Parte II). . . $\quad 88$

4.26 Coeficiente de pressão para a seção NLR7301 $\operatorname{com} M_{\infty}=0,752, \alpha=0,37^{\circ}$, usando esquema conservativo, com e sem correção viscosa. . . . . . . . . . 90

4.27 Espessura de deslocamento para a seção NLR7301 $\operatorname{com} M_{\infty}=0,752, \alpha=$ $0,37^{\circ}$, usando esquema conservativo. .

4.28 Correção da seção NLR7301, com $M_{\infty}=0,752$ e $\alpha=0,37^{\circ}$, usando a espessura de deslocamento. . . . . . . . . . . . . . . . . . . . . . . 91

4.29 Coeficiente de pressão estacionário, estacionário em-fase e estacionário forade-fase para a seção NLR7301 com $M_{\infty}=0,752, \alpha=0,37^{\circ}$, usando esquema conservativo, com correção viscosa. . . . . . . . . . . . . .

4.30 Distribuição de Mach no caso estacionário, estacionário em-fase e estacionário fora-de-fase na seção NLR7301 com $M_{\infty}=0,752, \alpha=0,37^{\circ}$, usando esquema conservativo, com correção viscosa. . . . . . . . . . . . . .

4.31 Distribuição da magnitude da variação no coeficiente de pressão para a seção NLR7301 com $M=0,752, \alpha=0,37^{\circ}$, oscilando em torno de $x / c=0,399$ com amplitude de $2,01^{\circ}$ e frequência reduzida de 0,4 , usando esquema não conservativo. 
4.32 Distribuição do ângulo de fase da variação no coeficiente de pressão para a seção NLR7301 $\operatorname{com} M=0,752, \alpha=0,37^{\circ}$, oscilando em torno de $x / c=$ 0,399 com amplitude de $2,01^{\circ}$ e frequência reduzida de 0,4 , usando esquema não conservativo.

4.33 Análise da parte estacionária do perfil NRL F5 em Mach 0,597, ângulo de ataque $=0,004^{\circ}, R e=4,57 \times 10^{6}$ e $\theta=0,115^{\circ}($ Parte I) . . . . . . . .

4.34 Análise da parte estacionária do perfil NRL F5 em Mach 0,597, ângulo de ataque $=0,004^{\circ}, \operatorname{Re}=4,57 \times 10^{6}$ e $\theta=0,115^{\circ}$ (Parte II) $\ldots \ldots \ldots$

4.35 Análise da parte em-fase do perfil NRL F5 em Mach 0,597, ângulo de ataque $=0,004^{\circ}, R e=4,57 \times 10^{6}$ e $\theta=0,115^{\circ}$ (Parte I).

4.36 Análise da parte em-fase do perfil NRL F5 em Mach 0,597, ângulo de ataque $=0,004^{\circ}, \operatorname{Re}=4,57 x 10^{6}$ e $\theta=0,115^{\circ}($ Parte II) $\ldots \ldots \ldots$

4.37 Análise da parte fora-de-fase do perfil NRL F5 em Mach 0,597, ângulo de ataque $=0,004^{\circ}, R e=4,57 \times 10^{6}$ e $\theta=0,115^{\circ}$ (Parte I).

4.38 Análise da parte fora-de-fase do perfil NRL F5 em Mach 0,597, ângulo de ataque $=0,004^{\circ}, R e=4,57 \times 10^{6}$ e $\theta=0,115^{\circ}$ (Parte II) . . . . . . .

4.39 Análise da parte estacionária do perfil NRL F5 em Mach 0,896, ângulo de ataque $=0,001^{\circ}, \operatorname{Re}=5,73 \times 10^{6}$ e $\theta=0,111^{\circ}($ Parte I $) \ldots \ldots \ldots$

4.40 Análise da parte estacionária do perfil NRL F5 em Mach 0,896, ângulo de ataque $=0,001^{\circ}, R e=5,73 \times 10^{6}$ e $\theta=0,111^{\circ}($ Parte II $) \ldots \ldots \ldots$

4.41 Análise da parte em-fase do perfil NRL F5 em Mach 0,896, ângulo de ataque $=0,001^{\circ}, R e=5,73 \times 10^{6}$ e $\theta=0,111^{\circ}$ (Parte I).

4.42 Análise da parte em-fase do perfil NRL F5 em Mach 0,896, ângulo de ataque $=0,001^{\circ}, R e=5,73 \times 10^{6}$ e $\theta=0,111^{\circ}$ (Parte II).

4.43 Análise da parte fora-de-fase do perfil NRL F5 em Mach 0,896, ângulo de ataque $=0,001^{\circ}, \operatorname{Re}=5,73 \times 10^{6}$ e $\theta=0,111^{\circ}($ Parte $\mathrm{I}) \ldots \ldots \ldots 106$

4.44 Análise da parte fora-de-fase do perfil NRL F5 em Mach 0,896, ângulo de ataque $=0,001^{\circ}, \operatorname{Re}=5,73 \times 10^{6}$ e $\theta=0,111^{\circ}$ (Parte II).

A.1 Seção do aerofólio NACA0012 . . . . . . . . . . . . . . . 111

A.2 Seção do aerofólio NACA0006. . . . . . . . . . . . . . . . . . 112

A.3 Seção do aerofólio NACA0015 . . . . . . . . . . . . . . . . 112 
A.4 Gráfico do residual usando TSD+Ent+Vort+CL para seção do perfil NACA0012 com $M_{\infty}=0$ e $\alpha=0,00^{\circ} \ldots \ldots \ldots 112$

A.5 Gráfico do residual usando TSD+Ent+Vort+CL para seção do perfil NACA0006 com $M_{\infty}=0$ e $\alpha=0,00^{\circ} \ldots \ldots \ldots \ldots$

A.6 Gráfico do residual usando TSD+Ent+Vort+CL para seção do perfil NACA0015 com $M_{\infty}=0$ e $\alpha=0,00^{\circ} \ldots \ldots \ldots 113$

A.7 Distribuição de $C_{p}$ para a seção NACA0012 com $M_{\infty}=0$ e $\alpha=0^{\circ}$. . . . 114

A.8 Distribuição de $C_{p}$ para a seção NACA0006 com $M_{\infty}=0$ e $\alpha=0^{\circ}$. . . . 115

A.9 Distribuição de $C_{p}$ para a seção NACA0015 $\operatorname{com} M_{\infty}=0$ e $\alpha=0^{\circ}$. . . . 115

A.10 Espessura de deslocamento para a seção NACA0012 com $M_{\infty}=0$ e $\alpha=0^{\circ} . \quad 116$

A.11 Espessura de deslocamento para a seção NACA0006 com $M_{\infty}=0$ e $\alpha=0^{\circ} .116$

A.12 Espessura de deslocamento para a seção NACA0015 com $M_{\infty}=0$ e $\alpha=0^{\circ} . \quad 117$

A.13 Gráfico do campo de velocidade em torno do perfil NACA0012 com $M_{\infty}=0$ e $\alpha=0^{\circ}$, usando TSD+Ent+Vort+CL . . . . . . . . . . . . . . . . . 117

A.14 Gráfico do campo de velocidade em torno do perfil NACA0006 com $M_{\infty}=0$ e $\alpha=0^{\circ}$, usando TSD+Ent+Vort+CL . . . . . . . . . . . . . . 118

A.15 Gráfico do campo de velocidade em torno do perfil NACA0015 com $M_{\infty}=0$ e $\alpha=0^{\mathrm{o}}$, usando TSD+Ent+Vort+CL . . . . . . . . . . . . . . . 118

A.16 Gráfico do potencial em torno do perfil NACA0012 com $M_{\infty}=0$ de entrada e $\alpha=0^{\circ}$, usando TSD+Ent+Vort+CL . . . . . . . . . . . . .

A.17 Gráfico do potencial em torno do perfil NACA0006 com $M_{\infty}=0$ de entrada e $\alpha=0^{\circ}$, usando TSD+Ent+Vort+CL. . . . . . . . . . . . . . . . . . 119

A.18 Gráfico do potencial em torno do perfil NACA0015 com $M_{\infty}=0$ de entrada e $\alpha=0^{\circ}$, usando TSD+Ent+Vort+CL . . . . . . . . . . . . . . . . . 120 


\section{Sumário}

1 Introdução 1

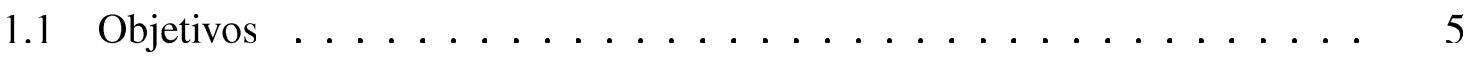

1.1.1 Organização do Trabalho . . . . . . . . . . . . . 6

2 Metodologia $\quad 7$

2.1 Equação de Pequenas Perturbações . . . . . . . . . . . . . . . . . . . 7

2.1 .1 Correção de Entropia . . . . . . . . . . . . . . . . . . . 12

2.1.2 Correção de Vorticidade . . . . . . . . . . . . . . 13

2.1.3 Correção Viscosa . . . . . . . . . . . . . . . . . . . . . . . . . . 14

2.2 Equação TSD no Domínio da Freqüência . . . . . . . . . . . . . . . . . 15

2.3 Condições de Contorno . . . . . . . . . . . . . . . . . . . . . . . 17

2.3.1 Condição de contorno na asa . . . . . . . . . . . . . . . . 17

2.3.2 Correção viscosa na condição de contorno na asa . . . . . . . . . 19

2.3.3 Condição de Contorno na Fronteira Externa . . . . . . . . . . . . . 21

2.4 Equações de Diferenças Finitas . . . . . . . . . . . . . . . . . . . . . 22

2.4.1 Sistema Governante . . . . . . . . . . . . . . . 22

2.4.2 Método de discretização . . . . . . . . . . . . . . 22

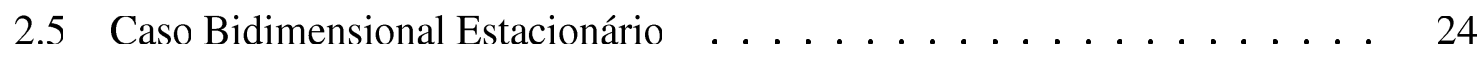

2.5.1 Equações Governantes . . . . . . . . . . . . . . . . . . . . . . . . 24

2.5.2 Detector de Regime Subsônico/Sônico/Supersônico . . . . . . . . . 24

2.5.3 Método de discretização . . . . . . . . . . . . . . . 26

2.5.4 Método Numérico f . . . . . . . . . . . . . . . . . . . 31

2.5.5 Esquematização da Malha . . . . . . . . . . . . . . . . 31

2.5.6 Condições de Contorno . . . . . . . . . . . . . . . . . . 33 
2.5.7 Refinamento e Dimensão da Malha . . . . . . . . . . . . . . . 35

3 Análise de Resultados $\quad 40$

3.1 Geometria dos Perfis . . . . . . . . . . . . . . . . 40

3.2 Critério de Convergência . . . . . . . . . . . . . . . 41

3.3 RAE2822 . . . . . . . . . . . . . . . . . . . 45

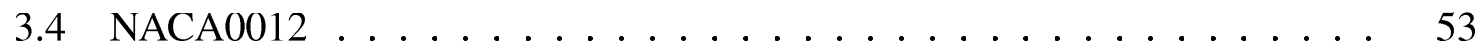

$3.5 \quad$ NLR7301 . . . . . . . . . . . . . . . . . . . 60

4 Programa UsTSD $\quad 65$

4.1 Critério de Convergência do Programa UsTSD . . . . . . . . . . . . . 65

4.2 Resultados do Programa UsTSD Estacionário . . . . . . . . . . . . . . . . 68

$4.2 .1 \quad$ Bidimensional . . . . . . . . . . . . . . . . . . 68

4.2 .2 Tridimensional . . . . . . . . . . . . . . . . . 80

4.3 Resultados do Programa UsTSD Não Estacionário . . . . . . . . . . . . . . . 89

4.3.1 Bidimensional . . . . . . . . . . . . . . . . . . . . 89

4.3 .2 Tridimensional . . . . . . . . . . . . . . . . . . . . . 94

5 Conclusões e Trabalhos Futuros $\quad 108$

5.1 Conclusão . . . . . . . . . . . . . . . . . . . . . . . . . . . 108

5.2 Sugestões para Trabalhos Futuros . . . . . . . . . . . . . . . . . . 109

A Resultados Bidimensionais Subsônicos

A.1 Perfis ......................... 111

A.2 Critério de Convergência . . . . . . . . . . . . . . . . . . . 112

A.3 Resultados . . . . . . . . . . . . . . . . . . . . . . . 114 


\section{Capítulo 1}

\section{Introdução}

Pode-se estudar a dinâmica dos fluidos de três maneiras: experimentalmente, analíticamente e computacionalmente. Esta terceira opção de estudo se tornou possível com a introdução dos computadores a partir dos anos 50, e é denominada de Dinâmica dos Fluidos Computacional (CFD - Computational Fluid Dynamics), área da computação que estuda métodos de simulação de fenômenos que envolvem fluidos em movimento ${ }^{[11]}$.

Uma das maiores vantagens do estudo experimental é que ela apresenta soluções mais realistas em relação aos outros métodos, porém há duas grandes razões que limitam este tipo de estudo: (a) o fenômeno em estudo nem sempre é passível de reprodução em laboratório, mesmo em escala reduzida; e (b) o custo e o tempo de montagem experimental podem ser muito mais altos em relação aos outros métodos.

O estudo analítico é mais geral, no qual é obtida uma solução fechada. No entanto, nem sempre as equações envolvidas no problema são passíveis a serem resolvidas analiticamente, necessitando-se assim de restrições (as quais levam a simplificações) para obter uma solução. Estas restrições nem sempre são aplicáveis a casos reais.

As maiores vantagens do $C F D$ são a flexibilidade das simulações, podendo-se trabalhar com geometrias complexas, e o custo operacional relativamente baixo, quando considerado que a maior necessidade de infra-estrutura é um bom computador. É claro que, dependendo da complexidade do problema a ser resolvido, o custo do computador adequado pode se tornar proibitivo. Em contra-partida, uma das desvantagens é a precisão da solução, pois às vezes o resultado produzido pode não condizer com os resultados físicos, já que a fidelidade computacional é comprometida pelas simplificações (discretizações/arrendondamentos). 
Parte da motivação para este trabalho deve-se aos recentes avanços em $C F D$ junto com o grande aumento na velocidade de processamento computacional. Com isso aumentou o interesse no estudo da aerodinâmica transônica (Mach entre 0,8 e 1,2) não-estacionária ${ }^{[17]}$. O estudo das características aeroelásticas neste regime já era de reconhecida importância desde a Segunda Guerra Mundial ${ }^{[13]}$.

A característica principal do escoamento transônico ${ }^{[18}$. é a existência de regiões supersônicas num escoamento livre subsônico. A região supersônica termina com uma onda de choque que, dependendo de sua intensidade, pode interagir significativamente com a camada-limite. Em geral, a camada-limite tende a se tornar mais espessa atrás do choque, movendo-o a montante. Se a onda de choque for suficientemente forte ela pode induzir a separação do escoamento.

Um dos problemas é que, nesta condição, o escoamento se torna bastante sensível a pequenas variações no ângulo de ataque e, devido ao acoplamento entre forças aerodinâmicas e a deformação elástica da estrutura, a aeronave se torna mais suscetível a instabilidades aeroelásticas.

No estudo do flutter transônico a interação entre choque e camada-limite pode, em muitos casos, ser desprezada. Isso ocorre porque o flutter pode ser estimado a partir de variações pequenas no ângulo de ataque, situação em que ondas de choque, inicialmente fracas, tendem a continuar fracas. Nesse caso o uso da equação potencial produz bons resultados, desde que seja corrigida para efeitos da variação da entropia e vorticidade. Isso já não é verdade para algumas seções de aerofólio que tendem a formar ondas de choque fortes mesmo com pequenos ângulos de ataque. Também pode ser interessante estudar o flutter em torno de grandes ângulos de ataque. No caso do estudo de oscilações de ciclo limite, em que a amplitude das oscilações pode ser consideravelmente grande, a interação entre choque e camada-limite não pode, em geral, ser desprezada.

Nos casos em que a interação entre choque e camada-limite é significativa torna-se necessário usar métodos que levem em conta os efeitos viscosos. Existem diversos métodos que corrigem a solução da equação potencial quando não há separação ou quando a separação é pequena e limitada a uma pequena região. Esses métodos produzem bons resultados quando comparados com métodos mais sofisticados que resolvem as equações de NavierStokes ou versões simplificadas desta (Navier-Stokes de camada-limite fina (Thin-Layer $N$ - 
$S$ ) ou Navier-Stokes com média de Reynolds (Reynolds Averaged $N-S)$ ). O custo computacional e problemas associados com a solução das equações de Navier-Stokes ainda são as principais motivações para o uso da equação potencial e da equação de Euler na solução de escoamentos transônicos não estacionários.

Os problemas aeroelásticos podem ser divididos em duas categorias. A primeira inclui os problemas estáticos em que forças aerodinâmicas e elásticas interagem. A deformação estrutural elástica atinge uma posição de equilíbrio ou diverge causando falhas. Divergência da asa e reversão de controles estão nesta categoria. A segunda categoria inclui os problemas dinâmicos em que, não só as forças aerodinâmicas e elásticas, mas também as forças de inércia interagem. Flutter, oscilações de ciclo limite e buffeting estão nesta categoria.

A utilização da aerodinâmica não estacionária é necessária no estudo de problemas de aeroelasticidade dinâmica em regime transônico. A solução para os problemas aeroelásticos pode ser obtida experimentalmente e teoricamente. Limitações nos recursos de mão-deobra, túneis de vento e o elevado custo associado com testes e fabricação de modelos, fazem com que ensaios experimentais aeroelásticos sejam muito caros e demorados. Testes em vôo também são necessários para validar testes em túnel de vento. Soluções teóricas podem reduzir custos, fornecendo resultados preliminares que sirvam como balizamento para os testes experimentais.

Em 1975, Magnus e Yoshihara ${ }^{23}$. mostraram que soluções para escoamentos transônicos bidimensionais e invíscidos podiam ser obtidas usando algoritmos de diferenças finitas. Foram obtidas características importantes do escoamento para um aerofólio com um flape oscilante (com a solução numérica das equações de Euler). Em 1977, Ballhaus e Goorjian ${ }^{[4}$. desenvolveram um programa de computador, o LTRAN2, que calculava com eficiência escoamentos transônicos não-estacionários para aerofólios em oscilações de baixa freqüência. O programa resolve a equação de pequenas perturbações bidimensional, não-linear e com baixas freqüências com um algoritmo implícito de diferenças finitas de direção alternada (ADI - Alternating-Direction Implicit).

Além dos métodos descritos acima, existe uma grande variedade de métodos numéricos de dinâmica dos fluidos que podem ser utilizados para a realização de estudos de aerodinâmica não-estacionária. Métodos que consideram efeitos não-lineares foram desenvolvidos usando a equação potencial completa (FPE - Full Potential Equation), a equação de peque- 
nas perturbações transônica (TSD - Transonic Small Disturbance), as equações de Euler (EE - Euler Equation), as equações de Navier-Stokes completa (FNS - Full Navier-Stokes), de camada limite fina (TLNS - Thin Layer Navier-Stokes), com média de Reynolds (RANS Reynolds Averaged Navier-Stokes) ou uma combinação destas. Estudos envolvendo essas equações são descritos a seguir.

\section{Equações de Navier-Stokes}

Steger e Bailey ${ }^{33}$. desenvolveram em 1980 um algoritmo computacional para resolver as equações de Navier-Stokes para aplicações aeroelásticas, este estudo mostrou o uso de um esquema de diferenças finitas implícito, usando uma solução com fatoração aproximada, para simular a vibração de um aileron em regime transônico num escoamento bidimensional. Chyu, Davis e Chang ${ }^{9}$. aplicaram a TLNS para calcular o escoamento transônico em torno de um aerofólio NACA 64A010, oscilando em torno do quarto-de-corda. Tang, Bartles, Chen e Liu ${ }^{34}$. descrevem uma investigação de oscilações de ciclo limite para um aerofólio supercrítico NLR 7301, resolvendo a TLNS através de integração no tempo e com técnica de computação paralela, e também concluem que os efeitos viscosos, incluindo modelagem da turbulência, têm um papel muito importante na previsão do desenvolvimento do ciclo limite e das ondas de choque. São apresentados dados de distribuição de pressão e de oscilação do perfil. Em geral, as equações de Navier-Stokes são muito difíceis de resolver, exigindo grandes quantidades de memória e tempo de computação. É necessária uma densidade muito alta da malha computacional para possibilitar cálculos bidimensionais e tridimensionais precisos para configurações realísticas.

\section{Equações de Euler $(E E)$}

Magnus e Yoshihara ${ }^{[23]}$ apresentaram o cálculo do escoamento transônico não-estacionário em torno de um aerofólio usando a representação por diferenças finitas das equações de Euler. Programas baseados na $E E$ para analisar uma asa retangular, com perfil supercrítico, com oscilações em arfagem foram então apresentados por Belk e Simpson ${ }^{[6]}$. E Bendiksen e Kousen ${ }^{[7]}$ estimaram, com sucesso, velocidades de flutter usando as $E E$ bidimensionais. Eles foram capazes de mostrar a queda na velocidade de flutter que ocorre em torno de Mach igual a 1 .

\section{Equação Potencial Completa (FPE)}

Até hoje a maior parte das estimativas numéricas para escoamentos transônicos não esta- 
cionários foram feitas resolvendo a FPE, que é derivada a partir das equações de Euler com as hipóteses de escoamento invíscido e irrotacional. A experiência tem mostrado que a FPE pode fornecer resultados bem próximos dos reais para várias condições de escoamento transônico e diferentes configurações. Malone e Sankar ${ }^{[24]}$ desenvolveram um algoritmo implícito de fatoração aproximada para resolver a FPE para escoamentos transônicos nãoestacionários. Um estudo mais recente, utilizando a FPE, foi conduzido por Schippers e Hounjet ${ }^{[32]}$. Eles desenvolveram dois métodos complementares, o primeiro método usa a integração no tempo da FPE e o outro é um método com linearização das equações no tempo para escoamentos transônicos bidimensionais.

\section{Equação Transônica de Pequenas Perturbações (TSD)}

Um dos problemas no cálculo de um escoamento transônico é que ele possui regiões subsônicas e supersônicas coexistindo, e a localização da fronteira entre as regiões não é conhecida a priori. Uma das soluções para este problema foi apresentada por Murman e Cole ${ }^{[26}$. com um algoritmo de diferenças finitas de tipo misto para resolver a equação TSD tanto para o escoamento subsônico (equação elíptica) quanto para o escoamento supersônico (equação hiperbólica). Soluções através da integração no tempo da equação TSD foram possibilitadas pelo algoritmo implícito de direção alternada $(A D I)$ introduzida no programa LTRAN2 desenvolvido por Ballhaus e Goorjian ${ }^{[4]}$. O programa LTRAN2 foi continuamente atualizado com uma série de aperfeiçoamentos, incluindo a adição de termos com a derivada no tempo nas condições de contorno proposta por Houwink e Vooren ${ }^{[19]}$, a adição de termos com a segunda derivada no tempo desenvolvida por Rizzetta e Chin ${ }^{[29]}$ e condições de contorno sem reflexão elaboradas por Kwak ${ }^{[21]}$. Em cálculos tridimensionais, Rizzetta e Borland ${ }^{[28}$. utilizaram o algoritmo ADI no programa XTRAN3S para obter a solução da TSD para asas isoladas.

\subsection{Objetivos}

A análise no domínio da freqüência apresenta vantagens significantes à analise no domínio do tempo. Na análise no domínio do tempo, o tamanho do passo tempo e o tempo total exigido para a análise pode impor um custo computacional muito maior do que a análise no domínio da freqüência ${ }^{8}$. , além disso há algumas técnicas que permitem transformações 
para o domínio da freqüência mantendo a não linearidade do problema. A técnica usada

neste estudo é a "média harmônica"[27], que mantém as condições não lineares dos termos no espaço mas assumindo uma variação harmônica nas variáveis dependentes do tempo.

Além disso, os dados obtidos das análises não-estacionárias no domínio do tempo são transformados no domínio da freqüência para poder observar e analisar um período da oscilação, com isso pode comprometer algumas características não lineares do problema.

O objetivo específico deste trabalho é a aplicação de métodos para correção de efeitos viscosos na solução da equação potencial de pequenas perturbações em regime transônico, no domínio da freqüência. Até hoje esses métodos de correção só foram implementados em estudos no domínio do tempo. O objetivo geral do estudo é o desenvolvimento de ferramentas visando, principalmente, as aplicações aeroelásticas como flutter, oscilações de ciclo limite e controle de vibrações.

\subsubsection{Organização do Trabalho}

É descrito, no Capítulo 2 a dedução da equação TSD e as correções de vorticidade, entropia e viscosidade; a equação TSD no domínio da freqüência e os métodos numéricos utilizados para resolução do projeto em estudo; e por fim são apresentadas as equações e discretizações para o caso bidimensional estacionário.

No capítulo 3, é feita uma análise dos resultados bidimensionais estacionários usando um programa computacional de pequenas perturbações (TSD), desenvolvido durante o doutorado e baseando na metodologia descrita no capítulo 2. Além disso, é feita uma comparação dos resultados obtidos numericamente (usando o programa TSD) com os dados experimentais obtidos da atual literatura.

Capítulo 4, são apresentados resultados de casos estacionários e não estcaionários (bidimensionais e tridimensionais) utilizando o programa UsTSD de pequenas perturbações tridimensional desenvolvido na Universidade de Kansas, sem e com correção viscosa (que foi implementada durante o doutorado). E estes resultados também são comparados com dados obtidos na atual literatura. Além disso, alguns resultados bidimensionais estacionários são comparados com os dados obtidos do programa TSD descrito no Capítulo 3.

Para finalizar, no capítulo 5, são apresentadas as conclusão e os possíveis estudos futuros. 


\section{Capítulo 2}

\section{Metodologia}

O problema geral da aeroelasticidade caracteriza-se pela interação entre a estrutura da aeronave e o escoamento aerodinâmico. No presente trabalho o interesse específico é estudar escoamentos viscosos transônicos não-estacionários com a presença das ondas de choque. As equações de Navier-Stokes são a representação matemática mais fiel do escoamento de fluidos com as características do ar. Porém, o custo computacional para resolvê-las pode se tornar proibitivo, tornando assim interessante o uso de representações mais simplificadas como a equação transônica de pequenas perturbações (TSD). Estas requerem um custo computacional muito menor e obtêm-se resultados satisfatórios na análise de uma série de fenômenos aerodinâmicos.

Nas seções a seguir são apresentadas a dedução e a formulação matemática das equações necessárias para o estudo proposto, juntamente com a teoria envolvida.

\subsection{Equação de Pequenas Perturbações}

A equação transônica de pequenas perturbações é derivada da equação potencial completa que, por sua vez, é derivada a partir das equações de Navier-Stokes, assumindo que o escoamento é isentrópico e irrotacional. Além disso, os fenômenos de transferência/condução de calor e forças de campo são desprezados. Assim a equação potencial completa pode ser escrita como $[15]$

$$
\begin{gathered}
\left(a^{2}-U^{2}\right) \Phi_{x x}+\left(a^{2}-V^{2}\right) \Phi_{y y}+\left(a^{2}-W^{2}\right) \Phi_{z z}-2 U V \Phi_{x y} \\
-2 U W \Phi_{x z}-2 V W \Phi_{y z}=\Phi_{t t}+2 U \Phi_{t x}+2 V \Phi_{t y}+2 W \Phi_{t z}
\end{gathered}
$$


onde

$a \quad$ é a velocidade do som local;

$U, V$ e $W \quad$ são as velocidades nas direções $x, y$ e $z$ respectivamente;

$\Phi \quad$ é o potencial de velocidade total;

$x, y$ e $z$ subscritos são as diferenciais nas coordenadas $x, y$ e $z$ respectivamente e

$t$ subscrito é a diferencial na coordenada do tempo.

O primeiro passo para obter a equação de pequenas perturbações é assumir que a componente $x$ da velocidade é a soma de uma velocidade não perturbada $V_{\infty}$ e uma componente de perturbação $\phi_{x}$, dada por

$$
\begin{array}{rlrl}
U & =\frac{\partial \Phi}{\partial x}=V_{\infty}+\frac{\partial \phi}{\partial x} & , & \frac{\partial \phi}{\partial x} \ll V_{\infty} ; \\
V=\frac{\partial \Phi}{\partial y}=\frac{\partial \phi}{\partial y} & , & \frac{\partial \phi}{\partial y} \ll V_{\infty} ; \\
W=\frac{\partial \Phi}{\partial z}=\frac{\partial \phi}{\partial z} & , & \frac{\partial \phi}{\partial z} \ll V_{\infty},
\end{array}
$$

sendo que

$$
\Phi(x, y, z, t)=V_{\infty} x+\phi(x, y, z, t) .
$$

A velocidade do som local ${ }^{[15]}$ é dada em termos de $a_{\infty}$, velocidade do som no escoamento livre, e do potencial de velocidade de perturbação

$$
a^{2} \approx a_{\infty}^{2}-(\gamma-1) \phi_{t}-\frac{1}{2}(\gamma-1)\left(\phi_{x}^{2}+2 V_{\infty} \phi_{x}+\phi_{y}^{2}+\phi_{z}^{2}\right)
$$

sendo que $\gamma$ é a razão do calor específico.

Eliminando os termos de maior ordem, a velocidade local do som é aproximada por

$$
a^{2} \approx a_{\infty}^{2}-(\gamma-1) \phi_{t}-(\gamma-1) V_{\infty} \phi_{x}
$$

Substituindo as equações (2.2) e (2.5) na equação (2.1) obtém-se

$$
\begin{array}{r}
{\left[a_{\infty}^{2}-(\gamma-1) \phi_{t}-(\gamma-1) V_{\infty} \phi_{x}-V_{\infty}^{2}-2 V_{\infty} \phi_{x}-\phi_{x}^{2}\right] \phi_{x x}} \\
+\left[a_{\infty}^{2}-(\gamma-1) \phi_{t}-(\gamma-1) V_{\infty} \phi_{x}-\phi_{y}^{2}\right] \phi_{y y} \\
+\left[a_{\infty}^{2}-(\gamma-1) \phi_{t}-(\gamma-1) V_{\infty} \phi_{x}-\phi_{z}^{2}\right] \phi_{z z} \\
-2\left(\phi_{x}+V_{\infty}\right) \phi_{y} \phi_{x y}-2\left(\phi_{x}+V_{\infty}\right) \phi_{z} \phi_{x z}-2 \phi_{y} \phi_{z} \phi_{y z} \\
=\phi_{t t}+2\left(\phi_{x}+V_{\infty}\right) \phi_{t x}+2 \phi_{y} \phi_{t y}+2 \phi_{z} \phi_{t z}
\end{array}
$$


Eliminando-se os termos de alta ordem tais como $\phi_{x} \phi_{y} \phi_{x y}$ e $(\gamma-1) \phi_{t} \phi_{x x}$ pois são muito pequenos comparados aos outros termos, e no lado direito da equação eliminando $\phi_{x} \phi_{t x}, \phi_{y} \phi_{t y}$ e $\phi_{z} \phi_{t z}$ pela mesma razão, obtém-se

$$
\begin{array}{r}
{\left[a_{\infty}^{2}-V_{\infty}^{2}-(\gamma+1) V_{\infty} \phi_{x}\right] \phi_{x x}+\left[a_{\infty}^{2}-(\gamma-1) V_{\infty} \phi_{x}\right] \phi_{y y}} \\
+\left[a_{\infty}^{2}-(\gamma-1) V_{\infty} \phi_{x}\right] \phi_{z z}-2 V_{\infty} \phi_{y} \phi_{x y}-2 V_{\infty} \phi_{z} \phi_{x z} \\
=\phi_{t t}+2 V_{\infty} \phi_{t x}
\end{array}
$$

Dividindo (2.7) por $a_{\infty}^{2}$, e lembrando que $M_{\infty}=\frac{V_{\infty}}{a_{\infty}}$, tem-se

$$
\begin{array}{r}
{\left[\left(1-M_{\infty}^{2}\right)-(\gamma+1) \frac{M_{\infty}^{2}}{V_{\infty}} \phi_{x}\right] \phi_{x x}+\left[1-(\gamma-1) \frac{M_{\infty}^{2}}{V_{\infty}} \phi_{x}\right] \phi_{y y}} \\
+\left[1-(\gamma-1) \frac{M_{\infty}^{2}}{V_{\infty}} \phi_{x}\right] \phi_{z z}-2 \frac{M_{\infty}^{2}}{V_{\infty}} \phi_{y} \phi_{x y}-2 \frac{M_{\infty}^{2}}{V_{\infty}} \phi_{z} \phi_{x z} \\
=\frac{\phi_{t t}}{a_{\infty}^{2}}+2 \frac{M_{\infty}^{2}}{V_{\infty}} \phi_{t x}
\end{array}
$$

Note que se $M_{\infty}$ estiver muito próximo de 1, o segundo termo do coeficiente que acompanha o termo $\phi_{x x}$ não poderá ser eliminado, caso isso ocorra ocorrerá haverá a perda dos fenômenos característicos na direção $x$ quando $M_{\infty}$ for 1 . Porém pode-se eliminar os ter$\operatorname{mos} \phi_{x} \phi_{y y}, \phi_{x} \phi_{z z}, \phi_{y} \phi_{x y}$ e $\phi_{z} \phi_{x z}$. Obtendo assim a forma clássica da Equação Transônica de Pequenas Perturbações:

$$
\left[\left(1-M_{\infty}^{2}\right)-(\gamma+1) \frac{M_{\infty}^{2}}{V_{\infty}} \phi_{x}\right] \phi_{x x}+\phi_{y y}+\phi_{z z}=\frac{\phi_{t t}}{a_{\infty}^{2}}+2 \frac{M_{\infty}^{2}}{V_{\infty}} \phi_{t x} .
$$

Observa-se que se $M_{\infty}=0$ e o escoamento estiver em regime estacionário, da equação (2.9) obtem-se a Equação de Laplace:

$$
\phi_{x x}+\phi_{y y}+\phi_{z z}=0
$$

Lembrando que os eixos $x, y$ e $z$ estão definidos como mostra a figura 2.1.

Para obter maior acurácia na equação (2.9) e considerando que exista enflechamento na asa, os termos não lineares, $2 M_{\infty}^{2} \phi_{y} \phi_{x y} / V_{\infty}$ e $(\gamma-1) M_{\infty}^{2} \phi_{x} \phi_{y y} / V_{\infty}$ são retidos na equação (2.8), eliminando assim apenas o termo $\phi_{x} \phi_{z z}$ e a adição e subtração do termo $(\gamma-1) \frac{M_{\infty}^{2}}{V_{\infty}} \phi_{y} \phi_{x y}$ é feita para se obter a equação $2.12, \operatorname{logo}$

$$
\begin{gathered}
{\left[\left(1-M_{\infty}^{2}\right) \phi_{x x}-(\gamma+1) \frac{M_{\infty}^{2}}{V_{\infty}} \phi_{x} \phi_{x x}-2 \frac{M_{\infty}^{2}}{V_{\infty}} \phi_{y} \phi_{x y}+(\gamma-1) \frac{M_{\infty}^{2}}{V_{\infty}} \phi_{y} \phi_{x y}\right]} \\
+\left[\phi_{y y}-(\gamma-1) \frac{M_{\infty}^{2}}{V_{\infty}} \phi_{x} \phi_{y y}-(\gamma-1) \frac{M_{\infty}^{2}}{V_{\infty}} \phi_{y} \phi_{x y}\right]+\phi_{z z}=\frac{\phi_{t t}}{a_{\infty}^{2}}+2 \frac{M_{\infty}^{2}}{V_{\infty}} \phi_{t x} .
\end{gathered}
$$




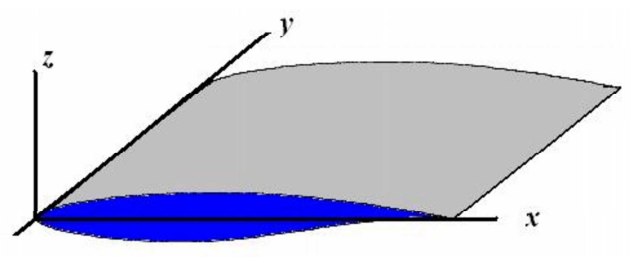

Figura 2.1: Definição dos eixos $x, y$ e $z$ em torno de um perfil.

Pode-se reescrever a equação (2.11) da seguinte forma:

$$
\begin{array}{r}
\frac{\partial}{\partial x}\left[\left(1-M_{\infty}^{2}\right) \phi_{x}-\frac{1}{2}(\gamma+1) \frac{M_{\infty}^{2}}{V_{\infty}} \phi_{x}^{2}+\frac{1}{2}(\gamma-3) \frac{M_{\infty}^{2}}{V_{\infty}} \phi_{y}^{2}\right] \\
+\frac{\partial}{\partial y}\left[\phi_{y}-(\gamma-1) \frac{M_{\infty}^{2}}{V_{\infty}} \phi_{x} \phi_{y}\right]+\frac{\partial}{\partial z}\left[\phi_{z}\right]=\frac{\partial}{\partial t}\left[\frac{\phi_{t}}{a_{\infty}^{2}}+2 \frac{M_{\infty}^{2}}{V_{\infty}} \phi_{x}\right] .
\end{array}
$$

Pode-se definir as variáveis adimensionais

$$
\hat{\phi}=\frac{\phi}{b_{0} V_{\infty}}, \quad \hat{t}=\frac{t}{\left(\frac{1}{\omega}\right)}, \quad \hat{x}=\frac{x}{b_{0}}, \quad \hat{y}=\frac{y}{b_{0}}, \quad \hat{z}=\frac{z}{b_{0}}
$$

sendo $b_{0}$ um comprimento de referência e $\omega$ a freqüência de oscilação. Lembrando que a freqüência reduzida é dada por $k=\frac{b_{0} \omega}{V_{\infty}}$. Substituindo as equações de (2.13) na equação (2.12), obtém-se

$$
\begin{array}{r}
\frac{V_{\infty}}{b_{0}}\left\{\frac{\partial}{\partial \hat{x}}\left[\left(1-M_{\infty}^{2}\right) \hat{\phi}_{\hat{x}}-\frac{1}{2}(\gamma+1) M_{\infty}^{2} \hat{\phi}_{\hat{x}}^{2}+\frac{1}{2}(\gamma-3) M_{\infty}^{2} \hat{\phi}_{\hat{y}}^{2}\right]\right\} \\
+\frac{V_{\infty}}{b_{0}}\left\{\frac{\partial}{\partial \hat{y}}\left[\hat{\phi}_{\hat{y}}-(\gamma-1) M_{\infty}^{2} \hat{\phi}_{\hat{x}} \hat{\phi}_{\hat{y}}\right]\right\}+\frac{V_{\infty}}{b_{0}}\left\{\frac{\partial}{\partial \hat{z}}\left[\hat{\phi}_{\hat{z}}\right]\right\} \\
=\omega\left\{\frac{\partial}{\partial \hat{t}}\left[k M_{\infty}^{2} \hat{\phi}_{\hat{t}}+2 M_{\infty}^{2} \hat{\phi}_{\hat{x}}\right]\right\} .
\end{array}
$$

Reorganizando a equação (2.14), tem-se

$$
\begin{array}{r}
\frac{\partial}{\partial \hat{x}}\left[\left(1-M_{\infty}^{2}\right) \hat{\phi}_{\hat{x}}-\frac{1}{2}(\gamma+1) M_{\infty}^{2} \hat{\phi}_{\hat{x}}^{2}+\frac{1}{2}(\gamma-3) M_{\infty}^{2} \hat{\phi}_{\hat{y}}^{2}\right] \\
+\frac{\partial}{\partial \hat{y}}\left[\hat{\phi}_{\hat{y}}-(\gamma-1) M_{\infty}^{2} \hat{\phi}_{\hat{x}} \hat{\phi}_{\hat{y}}\right]+\frac{\partial}{\partial \hat{z}}\left[\hat{\phi}_{\hat{z}}\right]=\frac{\partial}{\partial \hat{t}}\left[k M_{\infty}^{2} \hat{\phi}_{\hat{t}}+2 M_{\infty}^{2} \hat{\phi}_{\hat{x}}\right] .
\end{array}
$$


Definindo

$$
\begin{aligned}
A & =k^{2} M_{\infty}^{2}, \\
B & =2 k M_{\infty}^{2}, \\
E & =1-M_{\infty}^{2}, \\
F & =-\frac{1}{2}[\gamma+1] M_{\infty}^{2}, \\
G & =\frac{1}{2}[\gamma-3] M_{\infty}^{2}, \\
H & =-[\gamma-1] M_{\infty}^{2},
\end{aligned}
$$

e removendo-se o símbolo " $\wedge$ "das variáveis, a equação (2.15) fica na seguinte forma

$$
\left[E \phi_{x}+F \phi_{x}^{2}+G \phi_{y}^{2}\right]_{x}+\left[\phi_{y}+H \phi_{x} \phi_{y}\right]_{y}+\left[\phi_{z}\right]_{z}=\left[A \phi_{t}+B \phi_{x}\right]_{t}
$$

Pode-se reescrever a equação (2.16) como

$$
\frac{\partial f_{0}}{\partial t}+\frac{\partial f_{1}}{\partial x}+\frac{\partial f_{2}}{\partial y}+\frac{\partial f_{3}}{\partial z}=0
$$

onde

$$
\begin{aligned}
& f_{0}=-A \phi_{t}-B \phi_{x} ; \\
& f_{1}=E \phi_{x}+F \phi_{x}^{2}+G \phi_{y}^{2} ; \\
& f_{2}=\phi_{y}+H \phi_{x} \phi_{y} ; \\
& f_{3}=\phi_{z} .
\end{aligned}
$$

A equação (2.17) é conhecida como a forma conservativa da equação de pequenas perturbações $^{[5]}$. O coeficiente de pressão, $C_{p}$, em termos de pequenas perturbações é dado por ${ }^{[10]}$

$$
C_{p}=2 \phi_{t}-2 \phi_{x}
$$

Lembrando que para se obter a equação transônica de pequenas perturbações é considerado que o escoamento é isentrópico e irrotacional, logo para se obter uma solução mais próxima ao fenômeno físico é feito duas correções iniciais: correção de entropia e vorticidade. 


\subsubsection{Correção de Entropia}

A variação de entropia gerada pelo choque será modelada para modificar a equação TSD (Eq. 2.17). Esta modificação será denominada de "correção de entropia". Além desta correção será feita uma correção de entropia na fórmula da pressão. A correção de entropia ${ }^{[5}$. consiste em substituir a equação (2.18b) por

$$
f_{1}=(\gamma+1) M_{\infty}^{2} R\left(V \tilde{V}-\frac{1}{2} V^{2}\right)+G \phi_{y}^{2}
$$

onde

$$
\begin{aligned}
R & =\left[\frac{2+(\gamma-1) M_{\infty}^{2}}{(\gamma+1) M_{\infty}^{2}}\right]^{\frac{1}{2}} ; \\
V & =\frac{(1+R) \phi_{x}}{1+R+\phi_{x}} \\
\tilde{V} & =\left(\frac{R^{2}-1}{2 R}\right) .
\end{aligned}
$$

(Observação: Os termos $V$ e $\tilde{V}$, nesta sub-sessão, não representam a componente velocidade no eixo $y$.)

O primeiro termo deste novo $f_{1}$ é derivado da expansão assintótica da equação de Euler incluindo os efeitos da entropia que gera choque ${ }^{[12]}$. Esta correção de entropia foi escolhida, devido aos resultados numéricos apresentados em $\lceil 5\rceil$ que mostrou uma melhora da correlação entre o numérico e o experimental após a correção de entropia.

De forma análoga à dedução da equação (2.9), teremos que a Equação de Pequenas Perturbações com Correção de Entropia será dada por

$$
\xi \phi_{x x}+\phi_{y y}+\phi_{z z}=\frac{\phi_{t t}}{a_{\infty}^{2}}+2 \frac{M_{\infty}^{2}}{V_{\infty}} \phi_{t x}
$$

onde $\xi$ é a parte não homogênea da equação e é dada por

$$
\xi=(\gamma+1) M_{\infty}^{2} R\left[\tilde{V}\left(\frac{1+R}{1+R+\phi_{x}}\right)^{2}-V\left(\frac{1+R}{1+R+\phi_{x}}\right)^{2}\right] .
$$

Neste caso, é necessário fazer uma correção no coeficiente de pressão ${ }^{[5]}$. Assim a fórmula do $C_{p}$ incluindo a correção de entropia é dada por:

$$
C_{p}=C_{p_{i}}+C_{p_{s}}
$$


onde $C_{p_{i}}$ é o coeficiente de pressão isentrópico e $C_{p_{s}}$ é o coeficiente de pressão adicional devido à correção de entropia

$$
C_{p_{s}}=\frac{-2}{\gamma(\gamma-1) M_{\infty}^{2}} \frac{s}{c_{v}}
$$

Para realizar esta correção precisamos determinar a posição do choque e o salto de entropia através do choque. Essa posição corresponde ao lugar em que o escoamento passa de supersônico para subsônico na direção do escoamento. O salto é calculado usando a relação de Rankine-Hugoniot

$$
\frac{s}{c_{v}}=\ln \frac{(\gamma+1) u_{1}^{2}-(\gamma-1) R^{2}}{(\gamma+1) R^{2}-(\gamma-1) u_{1}^{2}}-\gamma \ln \frac{u_{1}^{2}}{R^{2}}
$$

onde

$$
u_{1}=1+\phi_{x}-u_{s}
$$

Na equação (2.27), $u_{1}$ é a velocidade imediatamente a montante do choque e $u_{s}$ é a velocidade de deslocamento do choque.

\subsubsection{Correção de Vorticidade}

O modelo de correção de vorticidade apresentado aqui, consiste em fazer modificações no vetor velocidade que, por sua vez, modificam a equação TSD (equação (2.17)) e a fórmula da pressão.

A correção de vorticidade consiste numa correção da componente de velocidade na direção do eixo $x$ (coerente com a hipótese de pequenas perturbações), na qual a perturbação $u=\phi_{x}$ é substituída por

$$
u=\phi_{x}-\frac{1}{\gamma(\gamma-1) M_{\infty}^{2}} \frac{s}{c_{v}}
$$

na equação (2.22).

A nova equação TSD após a correção de vorticidade, conserva a mesma forma da equação (2.17), pois a correção simplesmente consiste em fazer substituições nas equações (2.18a), (2.18b) e (2.18c) aplicando a equação (2.28).

Neste caso também precisa-se fazer uma correção no coeficiente de pressão ${ }^{[5]}$. Assim, a fórmula do $C_{p}$ incluindo a correção de entropia e vorticidade é dada por

$$
C_{p}=C_{p_{i}}+C_{p_{s}}+C_{p_{v}}
$$


onde $C_{p_{v}}$ é o coeficiente de correção da vorticidade para o coeficiente de pressão. A correção para $C_{p_{v}}$ tem o mesmo valor de $C_{p_{s}}$ só que em valor negativo ${ }^{[5]}$. Assim, elas são canceladas da equação (2.29). Logo, o coeficiente de pressão é dado por

$$
C_{p}=-2 \phi_{t}-2 \phi_{x}
$$

\subsubsection{Correção Viscosa}

O método consiste de um acoplamento iterativo entre a solução invíscida e uma técnica integral para a camada-limite que modela efeitos viscosos turbulentos. O acoplamento é feito através da distribuição de velocidade tangencial na superfície de sustentação, que é obtida do cálculo invíscido, e da espessura de deslocamento, $\delta^{*}$, que é obtida do cálculo da camada-limite.

As regiões da camada-limite onde não há separação são modeladas de modo quasiconstante utilizando-se as equações de lag-entrainment de Green. Os resultados do escoamento invíscido são utilizados na solução das equações da camada-limite que, por sua vez, servem para corrigir as condições de contorno para um novo cálculo invíscido. As condições de contorno corrigidas pelos efeitos da viscosidade, que são modificadas pela solução da camada-limite, são dadas por:

$$
\delta_{z}^{ \pm}=S_{x}^{ \pm}+S_{t}^{ \pm}+\delta_{x}^{ \pm}, \quad x_{h a} \leq x \leq x_{b f}, \quad z=0^{ \pm}
$$

onde o sobrescrito \pm refere-se às superfícies superior e inferior do aerofólio, $S$ representa as coordenadas do aerofólio, os subscritos $b a$ e $b f$ referem-se ao bordo de ataque e bordo de fuga respectivamente. A equação (2.31) não inclui $\delta_{t}^{*}$, devido à hipótese de condição quasi-constante no tempo.

Para análise das regiões onde há leve separação as equações da camada-limite são escritas em forma inversa. Nesse caso as equações da camada-limite são resolvidas a partir de uma distribuição de espessura de deslocamento especificada. A solução das equações inversas (a velocidade na extremidade da camada-limite) é usada então para atualizar a espessura de deslocamento através do método de Carter ${ }^{[37]}$ :

$$
\delta_{\text {novo }}=\delta_{\text {anterior }}+\varepsilon \delta_{\text {anterior }}\left(\frac{U_{i}}{U_{v}}-1\right)
$$


onde $\varepsilon$ é um fator de relaxamento, $U_{i}$ é a velocidade invíscida na extremidade da camadalimite, e $U_{v}$ é a velocidade viscosa na extremidade da camada-limite. Esse procedimento é repetido até que se atinja convergência.

\subsection{Equação TSD no Domínio da Freqüência}

Para resolver a equação TSD no domínio da freqüência usa-se o método de média harmônica, a qual é usada para resolver problemas de equações diferenciais periódicas nãolineares $[20],[30]$. A aplicação deste método consiste em assumir que a solução da equação $T S D$ é da seguinte forma:

$$
\phi(x, y, z, t)=\phi^{s}(x, y, z)+\phi^{u}(x, y, z) \cos (\theta+\delta)
$$

onde $\phi^{s}$ e $\phi^{u}$ são, respectivamente, as componentes estacionária e não-estacionária da perturbação no potencial de velocidade $\theta$ é o ângulo de fase do movimento das superfíces aerodinâmicas e $\delta$ é o ângulo diferença de fase (atraso) entre o movimento e a resposta aerodinâmica. O método da média harmônica será aplicado à equação (2.16). Para isso, são desenvolvidas os seguintes passos:

1. Substituindo a equação (2.33) na equação (2.16), temos:

$$
\begin{array}{r}
\frac{\partial}{\partial x}\left[E\left(\phi_{x}^{s}+\phi_{x}^{u} \cos (\theta+\delta)\right)+F\left(\phi_{x}^{s}+\phi_{x}^{u} \cos (\theta+\delta)\right)^{2}+G\left(\phi_{y}^{s}+\phi_{y}^{u} \cos (\theta+\delta)\right)^{2}\right] \\
+\frac{\partial}{\partial y}\left[\left(\phi_{y}^{s}+\phi_{y}^{u} \cos (\theta+\delta)\right)+H\left(\phi_{x}^{s}+\phi_{x}^{u} \cos (\theta+\delta)\right)\left(\phi_{y}^{s}+\phi_{y}^{u} \cos (\theta+\delta)\right)\right] \\
+\frac{\partial}{\partial z}\left[\left(\phi_{z}^{s}+\phi_{z}^{u} \cos (\theta+\delta)\right)\right]=-A \phi^{u} \cos (\theta+\delta)-B \phi_{x}^{u} \operatorname{sen}(\theta+\delta)
\end{array}
$$

2. Obtém-se a equação (2.34) para o caso de escoamento estacionário ao descartar-se os termos não-estacionários

$$
\frac{\partial}{\partial x}\left[E \phi_{x}^{s}+F\left(\phi_{x}^{s}\right)^{2}+G\left(\phi_{y}^{s}\right)^{2}\right]+\frac{\partial}{\partial y}\left[\phi_{y}^{s}+H \phi_{x}^{s} \phi_{y}^{s}\right]+\frac{\partial}{\partial z}\left[\phi_{z}^{s}\right]=0 .
$$

3. Integrando a equação (2.34) num círculo unitário e subtraindo a equação do escoamento estacionário, isto é, $\int_{0}^{2 \pi}[E q .(2.34)] d \theta-2 \pi[E q .(2.35)]$, obtém-se a média total da oscilação, dada por:

$$
\frac{\partial}{\partial x}\left[F\left(\phi_{x}^{u}\right)^{2}+G\left(\phi_{y}^{u}\right)^{2}\right]+\frac{\partial}{\partial y}\left[H \phi_{x}^{u} \phi_{y}^{u}\right]=0
$$


4. Multiplicando a equação (2.34) por $\cos \theta$ e integrando num círculo unitário, isto é $\int_{0}^{2 \pi}[E q \cdot(2.34)] \cdot[\cos \theta] d \theta$, obtém-se a média em-fase, dada por:

$$
\begin{array}{r}
-A \phi^{u(i)}-B \phi_{x}^{u(o)}=\frac{\partial}{\partial x}\left[E \phi_{x}^{u(i)}+2 F \phi_{x}^{s} \phi_{x}^{u(i)}+2 G \phi_{y}^{s} \phi_{y}^{u(i)}\right] \\
+\frac{\partial}{\partial y}\left[\phi_{y}^{u(i)}+H \phi_{x}^{s} \phi_{y}^{u(i)}+H \phi_{y}^{s} \phi_{x}^{u(i)}\right]+\frac{\partial}{\partial z}\left[\phi_{z}^{u(i)}\right] .
\end{array}
$$

onde $\phi^{u(i)}=\phi^{u} \cos \delta$ e $\phi^{u(o)}=\phi^{u} \operatorname{sen} \delta$.

5. Multiplicando a equação (2.34) por $\operatorname{sen} \theta$ e integrando num círculo unitário, isto é $\int_{0}^{2 \pi}[E q \cdot(2.34)] \cdot[\operatorname{sen} \theta] d \theta$, obtém-se a média fora-de-fase, dada por:

$$
\begin{gathered}
A \phi^{u(o)}-B \phi_{x}^{u(i)}=\frac{\partial}{\partial x}\left[E \phi_{x}^{u(o)}+2 F \phi_{x}^{s} \phi_{x}^{u(o)}+2 G \phi_{x}^{s} \phi_{y}^{u(o)}\right] \\
+\frac{\partial}{\partial y}\left[\phi_{y}^{u(o)}+H \phi_{x}^{s} \phi_{y}^{u(o)}+H \phi_{y}^{s} \phi_{x}^{u(o)}\right]+\frac{\partial}{\partial z}\left[\phi_{z}^{u(o)}\right]
\end{gathered}
$$

6. Multiplicando a equação (2.36) por $\cos ^{2} \delta$ e somando as equações (2.35) e (2.37), obtém-se a equação em-fase, dada por

$$
\begin{aligned}
-A \phi^{u(i)}-B \phi_{x}^{u(o)} & =\frac{\partial}{\partial x}\left[E \phi_{x}^{(i)}+F\left(\phi_{x}^{(i)}\right)^{2}+G\left(\phi_{y}^{(i)}\right)^{2}\right] \\
& +\frac{\partial}{\partial y}\left[\phi_{y}^{(i)}+H \phi_{x}^{(i)} \phi_{y}^{(i)}\right]+\frac{\partial}{\partial z}\left[\phi_{z}^{(i)}\right] .
\end{aligned}
$$

onde $\phi^{(i)}=\phi^{s}+\phi^{u(i)}$.

7. Similarmente, multiplica-se a equação (2.36) por $\operatorname{sen}^{2} \delta$ em vez de $\cos ^{2} \delta$ e somando as equações (2.35) e (2.38), obtém-se a equação fora-de-fase, dada por:

$$
\begin{aligned}
A \phi^{u(o)}-B \phi_{x}^{u(i)} & =\frac{\partial}{\partial x}\left[E \phi_{x}^{(o)}+F\left(\phi_{x}^{(o)}\right)^{2}+G\left(\phi_{y}^{(o)}\right)^{2}\right] \\
+ & \frac{\partial}{\partial y}\left[\phi_{y}^{(o)}+H \phi_{x}^{(o)} \phi_{y}^{(o)}\right]+\frac{\partial}{\partial z}\left[\phi_{z}^{(o)}\right] .
\end{aligned}
$$

onde $\phi^{(o)}=\phi^{s}+\phi^{u(o)}$

Tem-se que reescrevendo o $C_{p}$, dado pela equação (2.19), usando o método de média harmônica e repetindo os passos de 1 a 7 para se obter as equações (2.39) e (2.40) obtém-se:

1. $C_{p}$ em-fase:

$$
C_{p}^{u(i)}=-2 \phi_{x}^{u(i)}+2 k \phi^{u(o)} ;
$$

2. $C_{p}$ fora-de-fase:

$$
C_{p}^{u(o)}=-2 \phi_{x}^{u(o)}+2 k \phi^{u(i)} .
$$




\subsection{Condições de Contorno}

\subsubsection{Condição de contorno na asa}

Assumindo que a superfície da asa possa ser descrita como

$$
z=z(x, y, t)
$$

onde $z$ é a altura do aerofólio no plano $(z=0)$, da superfície da asa, isto é como pode-se observar na Figura 2.2.

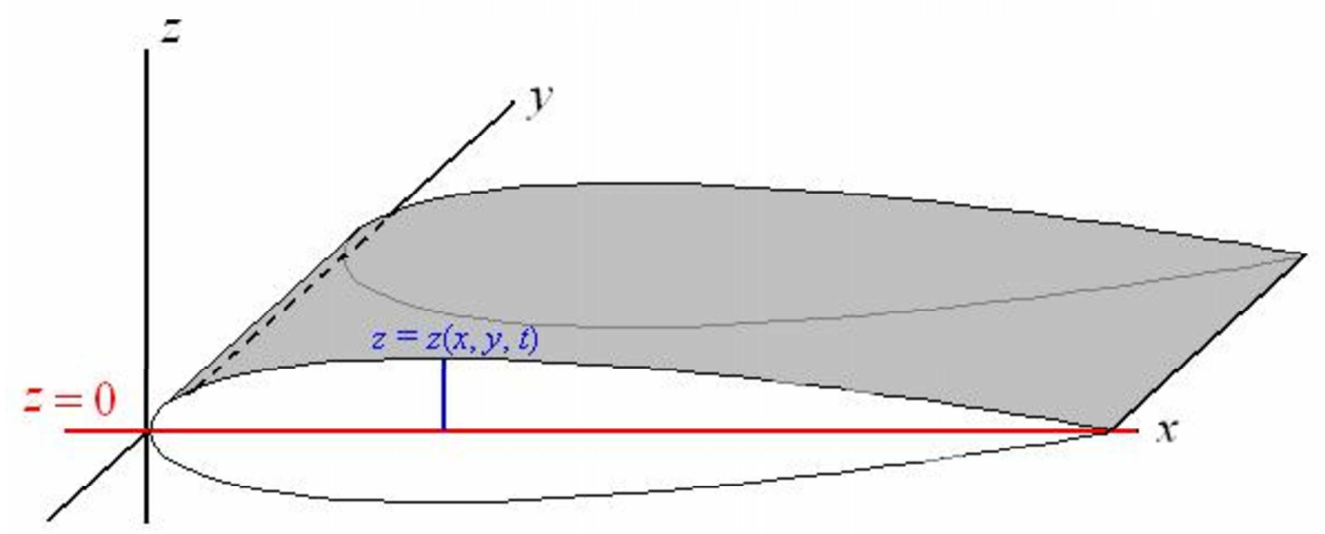

Figura 2.2: Sistema de coordenadas usado para definir a geometria das superfícies de sustentação.

A condição de contorno da asa é definida tal que a velocidade normal do fluido na superfície da asa seja igual a velocidade normal da superfície (transpiração igual a zero). Usando o sistema de coordenadas $(X, Y, Z)$ fixado no campo de escoamento, a condição de escoamento tangente, $F$, é dada por

$$
\frac{D F}{D t}=\frac{\partial F}{\partial t}+\vec{V} \cdot \nabla F=0
$$

onde

$$
F=Z-f(X, Y, t)
$$

e $f(X, Y, 0, t)$ a localização na superfície do perfil. Reescrevendo a equação (2.44), temos

$$
\frac{\partial f}{\partial t}+U \frac{\partial f}{\partial X}+V \frac{\partial f}{\partial Y}=W
$$


Para concordar com o domínio da freqüência temos que a velocidade potencial é dada por

$$
\Phi=X V_{\infty} \cos \alpha+Z V_{\infty} \operatorname{sen} \alpha+\phi(X, Y, Z, t)
$$

e, em concordância com a teoria de pequenas perturbações, pode-se considerar que $\cos \alpha=1$ e $\operatorname{sen} \alpha=0, \log 0$

$$
\Phi \approx X V_{\infty}+Z V_{\infty} \alpha+\phi
$$

As componentes de velocidades são expressas como

$$
\begin{aligned}
& U=V_{\infty}+\phi_{X} ; \\
& V=\phi_{Y} ; \\
& W=V_{\infty} \alpha+\phi_{Z} .
\end{aligned}
$$

Substituindo as equações (2.48) e (2.49) na equação (2.46), tem-se

$$
\frac{\partial f}{\partial t}+\left(V_{\infty}+\phi_{X}\right) \frac{\partial f}{\partial X}+\phi_{Y} \frac{\partial f}{\partial Y}=V_{\infty} \alpha+\phi_{Z}
$$

Linearizando a equação acima, isto é, desprezando os termos não-lineares, obtém-se então

$$
\phi_{Z}=\frac{\partial f}{\partial t}+V_{\infty}\left(\frac{f}{X}-\alpha\right)
$$

Usando o mesmo processo de adimensionalização da equação $T S D$, tem-se que a equação (2.51) é reescrita na seguinte forma

$$
\phi_{Z}(X, Y, 0, t)=f_{X}(X, Y, 0, t)+k f_{t}(X, Y, 0, t)-\alpha
$$

A condição de contorno é modificada para incluir a escala do tipo-Krupp ${ }^{[16]}$, para o efeito da compressibilidade com Mach próximo de 1. Assim a equação acima é reescrita como o seguinte

$$
\phi_{Z}(X, Y, 0, t)=\frac{1}{M_{\infty}^{0.25}}\left[f_{X}(X, Y, 0, t)+k f_{t}(X, Y, 0, t)-\alpha\right] .
$$

Para uma asa em oscilação, a localização na superfície do perfil $f(X, Y, 0, t)$ pode ser descrita por ${ }^{[16]}$

$$
f(X, Y, \pm 0, t)=-f^{v}(x, y) \cos \theta(t)+f^{s}(x, y) \cos \alpha^{*}(t)-\left(x-x_{p}\right) \operatorname{sen} \alpha^{*}(t)
$$


onde $x, y$ são calculados no sistema de coordenadas local, que se movimenta com a superfície aerodinâmica; $\alpha^{*}$ é o ângulo de ataque num determinado instante de tempo; $f^{v}$ é o deslocamento da superfície aerodinâmica em oscilação; $f^{s}$ é a forma da superfície aerodinâmica.

A equação (2.54) é uma expressão geral a qual inclui o movimento de arfagem da superfície rígida e o movimento devido à deformação elástica da superfície (para análise aeroelástica). A transformação entre os sistemas de coordenadas fixo e em movimento é dado por

$$
X=\left(x-x_{p}\right) \cos \alpha^{*}+f^{s}(x, y) \operatorname{sen} \alpha^{*}+x_{p}
$$

onde $x_{p}$ representa a posição do centro de arfagem. O ângulo de ataque, $\alpha^{*}(t)$, em qualquer instante de tempo pode ser expresso por

$$
\alpha^{*}(t)=\bar{\alpha} \cos \theta
$$

Substituindo as equações (2.54) a (2.56) na equação (2.53) e usando a técnica de média harmônica, as condições de contorno na superfície da asa podem ser escritas como:

- estacionário:

$$
\phi_{z}^{s}=\frac{1}{M_{\infty}^{0.25}}\left(f_{x}^{s}\right)
$$

- em-fase:

$$
\phi_{z}^{(i)}=\frac{1}{M_{\infty}^{0.25}}\left(f_{x}^{s}-\alpha_{s}-\bar{\alpha}-f_{x}^{v}\right)
$$

- fora-de-fase:

$$
\phi_{z}^{(o)}=\frac{1}{M_{\infty}^{0.25}}\left\{f_{x}^{s}-\alpha_{s}-k\left[f^{v}+\left(x-x_{p}\right) \bar{\alpha}\right]\right\}
$$

\subsubsection{Correção viscosa na condição de contorno na asa}

Seja $\delta^{*}$ a espessura de deslocamento. Assumindo que a solução $\delta^{*}$ seja periódica (no tempo), pode-se escrever

$$
\delta^{*}=\delta^{* s}+\delta^{* u} \cos (\theta+\delta)
$$

Substituindo as equações (2.54) e (2.56) na equação (2.53) e aplicando a correção viscosa, temos:

$$
\begin{array}{r}
\phi_{Z}^{s}+\phi_{Z}^{u} \cos (\theta+\delta)=\frac{1}{M_{\infty}^{0,25}}\left\{-f_{x}^{v} \cos \theta+\left[f_{x}^{s}+\delta_{x}^{* s}+\delta_{x}^{* u} \cos (\theta+\delta)\right]-\bar{\alpha} \cos \theta\right. \\
\left.+k f^{v} \operatorname{sen} \theta-k \delta^{* u} \operatorname{sen}(\theta+\delta)+\left(x-x_{p}\right) \bar{\alpha} \operatorname{sen} \theta-\alpha_{s}\right\}
\end{array}
$$


Usando a técnica de média harmônica, as condições de contorno na asa são dadas por:

- a equação estacionária é dada por:

$$
\phi_{z}^{s}=\frac{1}{M_{\infty}^{0,25}}\left\{f_{x}^{s}+\delta_{x}^{* s}-\alpha_{s}\right\}
$$

- equação em-fase:

$$
\phi_{z}^{(i)}=\frac{1}{M_{\infty}^{0.25}}\left(f_{x}^{s}-\alpha_{s}-\bar{\alpha}-f_{x}^{v}+\delta_{x}^{*(i)}+k \delta^{*(o)}-k \delta^{* s}\right)
$$

onde $\delta^{*(i)}=\delta^{* s}+\delta^{* u} \cos \delta, \delta^{*(o)}=\delta^{* s}+\delta^{* u} \sin \delta$ e $\phi_{z}^{(i)}=\phi_{z}^{s}+\phi_{z}^{u} \cos \delta$;

- equação fora-de-fase:

$$
\phi_{z}^{(o)}=\frac{1}{M_{\infty}^{0.25}}\left\{f_{x}^{s}-\alpha_{s}-k\left[f^{v}+\left(x-x_{p}\right) \bar{\alpha}\right]+\delta_{x}^{*(o)}+k \delta^{*(i)}-k \delta^{* s}\right\} .
$$

onde $\phi_{z}^{(o)}=\phi_{z}^{s}+\phi_{z}^{u} \operatorname{sen} \delta$

Da forma como o método está implementado até o momento, a correção viscosa é aplicada de maneira uniforme ao longo de toda a envergadura da superfície de sustentação. A correção é baseada na distribuição de Mach da seção no plano de simetria e o mesmo valor é aplicado em toda a envergadura. Essa correção funciona bem para escoamentos bidimensionais já que não há variação da distribuição de Mach ao longo da envergadura. No entanto, para escoamentos tridimensionais é necessário obter uma correção viscosa para cada seção da superfície de sustentação. Essa modificação, apesar de não impor grandes dificuldades, ainda não foi implementada no programa TSD+Ent+Vort+CL. Resumindo, o processo de correção viscosa para asas tridimensionais é o seguinte:

- A distribuição de Mach é obtida para cada seção da asa;

- A espessura de deslocamento da camada limite é calculada para cada seção da asa com base na distribuição de Mach;

- A geometria da asa é modificada adicionando a espessura de deslocamento de cada seção à geometria original;

- A iteração seguinte para o escoamento invíscido considera a geometria modificada da asa; - O processo é repetido até a convergência da solução.

Em relação à análise não estacionária o processo será o mesmo descrito na seção anterior: 
- É obtida a solução estacionária com a correção viscosa;

- As soluções em-fase e fora-de-fase são obtidas com base na solução estacionária com a correção viscosa.

Acredita-se que com a correção feita dessa forma a simulação estará se aproximando mais do fenômeno físico real. Resultados de flutter e oscilações de ciclo limite disponíveis em publicações terão que ser usados para validar a implementação da coreção viscosa.

\subsubsection{Condição de Contorno na Fronteira Externa}

As fronteiras externas da região de domínio no espaço tridimensional são dadas conforme mostra a Figura 2.3:

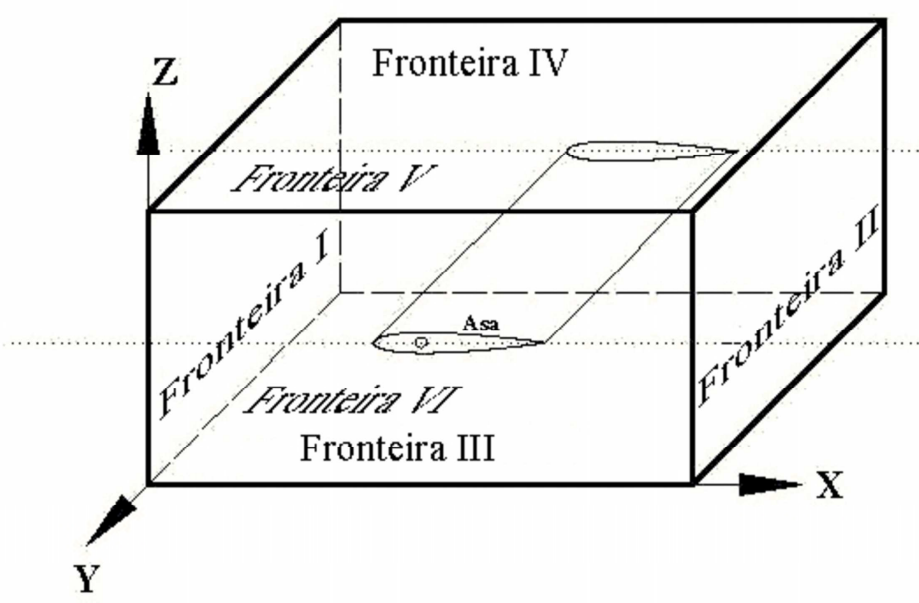

Figura 2.3: Definição das fronteiras externas do domínio computacional.

As condições de contorno de cada fronteira externa são:

- Fronteira I e II: as velocidades dos escoamentos de entrada e saída são iguais à velocidade do escoamento livre, isto é, a componente de velocidade $U=V_{\infty}, \phi_{X}=0$.

- Fronteira III, V e VI: a condição utilizada zqui é que houvesse apenas a componente de velocidade $\mathrm{U}$, ou seja, a componente de velocidade transversal $V=0, \phi_{Y}=0$. Podese utilizar a condição não refletora, que visa principalmente, evitar a reflexão de ondas de choque nas paredes das fronteiras computacionais.

- Fronteira IV: pode se usada uma condição de simetria ou condição de parede não refletora. 
(Observação: As condições da Fronteira III e IV são diferentes, pois é assumido que a Fronteira III esteja próximo a fuselagem ou a raiz do perfil.)

\subsection{Equações de Diferenças Finitas}

\subsubsection{Sistema Governante}

O sistema governante é decomposta nas equações (2.35), (2.39) e (2.40). Reescrevendoas respectivamente tem-se:

- a equação estacionária:

$$
\left(E+2 F \phi_{x}^{s}\right) \phi_{x x}^{s}+\left(1+H \phi_{x}^{s}\right) \phi_{y y}^{s}+(2 G+H) \phi_{y}^{s} \phi_{x y}^{s}+\phi_{z z}^{s}=0
$$

- a equação em-fase:

$$
\left(E+2 F \phi_{x}^{(i)}\right) \phi_{x x}^{(i)}+D \phi_{y}^{(i)} \phi_{x y}^{(i)}+\left(1+H \phi_{x}^{(i)}\right) \phi_{y y}^{(i)}+\phi_{z z}^{(i)}+A \phi^{u(i)}+B \phi_{x}^{u(o)}=0
$$

- a equação fora-de-fase:

$$
\left(E+2 F \phi_{x}^{(o)}\right) \phi_{x x}^{(o)}+D \phi_{y}^{(o)} \phi_{x y}^{(o)}+\left(1+H \phi_{x}^{(o)}\right) \phi_{y y}^{(o)}+\phi_{z z}^{(o)}+A \phi^{u(o)}-B \phi_{x}^{u(i)}=0
$$

onde

$$
\begin{aligned}
& A=k^{2} M_{\infty}^{2} ; \\
& B=2 k M_{\infty}^{2} ; \\
& D=-2 M_{\infty}^{2} ; \\
& E=1-M_{\infty}^{2} ; \\
& F=-\frac{1}{2}[\gamma+1] M_{\infty}^{2} ; \\
& H=-[\gamma-1] M_{\infty}^{2} .
\end{aligned}
$$

\subsubsection{Método de discretização}

As derivadas parciais dos potenciais de velocidade podem ser representadas por diferenças finitas. No regime transônico, o escoamento possui regiões com escoamento subsônico e 
com escoamento supersônico. Por Murman e Cole ${ }^{[26]}$, tem-se que o tipo diferenciação deve mudar de acordo com o regime do escoamento local. A determinação do regime local do escoamento é feita usando dados da iteração anterior. Se o escoamento é subsônico no ponto a ser analisado, as formulações de diferenças finitas para as derivadas de primeira ordem são

$$
\begin{aligned}
\phi_{x} & =\frac{1}{2}\left(\frac{\phi_{i+1 j k}-\phi_{i j k}}{x_{i+1}-x_{i}}+\frac{\phi_{i j k}-\phi_{i-1 j k}}{x_{i}-x_{i-1}}\right) ; \\
\phi_{y} & =\frac{1}{2}\left(\frac{\phi_{i j+1 k}-\phi_{i j k}}{y_{j+1}-y_{j}}+\frac{\phi_{i j k}-\phi_{i j-1 k}}{y_{j}-y_{j-1}}\right) ; \\
\phi_{z} & =\frac{1}{2}\left(\frac{\phi_{i j k+1}-\phi_{i j k}}{z_{k+1}-z_{k}}+\frac{\phi_{i j k}-\phi_{i j k-1}}{z_{k}-z_{k-1}}\right) ;
\end{aligned}
$$

onde os sub-índices $i, j, k$ identificam um ponto na $i, j, k$-ésima posição nos eixos $x, y, z$. respectivamente na malha tridimensional. Estas diferenças fnitas não são de segunda ordem, visto que o espaçamento não é uniforme.

As formulações de diferenças finitas para as derivadas de segunda ordem neste caso são[3]:

$$
\begin{aligned}
& \phi_{x x}=\frac{2}{x_{i+1}-x_{i-1}}\left(\frac{\phi_{i+1 j k}-\phi_{i j k}}{x_{i+1}-x_{i}}-\frac{\phi_{i j k}-\phi_{i-1 j k}}{x_{i}-x_{i-1}}\right) ; \\
& \phi_{y y}=\frac{2}{y_{j+1}-y_{j-1}}\left(\frac{\phi_{i j+1 k}-\phi_{i j k}}{y_{j+1}-y_{j}}-\frac{\phi_{i j k}-\phi_{i j-1 k}}{y_{j}-y_{j-1}}\right) ; \\
& \phi_{z z}=\frac{2}{z_{k+1}-z_{k-1}}\left(\frac{\phi_{i j k+1}-\phi_{i j k}}{z_{k+1}-z_{k}}-\frac{\phi_{i j k}-\phi_{i j k-1}}{z_{k}-z_{k-1}}\right) ;
\end{aligned}
$$

Estas diferenças são centrais. Com relação à derivada mista $\phi_{x y}$, tem-se:

$$
\phi_{x y}=\frac{1}{2}\left(\frac{\phi_{x_{i j+1 k}}-\phi_{x_{i j k}}}{y_{j+1}-y_{j}}+\frac{\phi_{x_{i j k}}-\phi_{x_{i j-1 k}}}{y_{j}-y_{j-1}}\right)
$$

sendo que $\phi_{x_{i}, k}$ é igual a $\phi_{x}$ no ponto na $i, j, k$-ésima posição, dada pela equação (2.68).

No caso do escoamento ser supersônico no ponto a ser analisado, utiliza-se diferença atrasada na direção $x$ para $\phi_{x}, \phi_{x x}$ e $\phi_{x y}$, ou seja, as que têm dependência no eixo $x$, que são dadas por

$$
\begin{gathered}
\phi_{x}=\frac{\phi_{i j k}-\phi_{i-1 j k}}{x_{i}-x_{i-1}} ; \\
\phi_{x x}=\frac{1}{x_{i}-x_{i-1}}\left(\frac{\phi_{i j k}-\phi_{i-1 j k}}{x_{i}-x_{i-1}}-\frac{\phi_{i-1 j k}-\phi_{i-2 j k}}{x_{i-1}-x_{i-2}}\right) ;
\end{gathered}
$$




$$
\phi_{x y}=\frac{1}{2}\left(\frac{\phi_{x_{i} j+1 k}-\phi_{x_{i j k}}}{y_{j+1}-y_{j}}+\frac{\phi_{x_{i j k}}-\phi_{x_{i j-1 k}}}{y_{j}-y_{j-1}}\right) ;
$$

onde $\phi_{x_{i j k}}=\phi_{x}$ é dada na equação (2.75). Estas formulações serão aplicadas nas equações (2.65) a (2.67). A seguir usa-se um método de solução numérica $S L O R$ para resolver este sistema de equações.

Vejamos inicialmente o método chamado SOR (sucessive over-relaxation), um método iterativo que usa sucessivas aproximações através de diferenças entre um valor atual e o anterior. Para controlar essa diferença usa-se um fator de relaxação que pode aumentar ou diminuir essa diferença, assim podendo acelerar ou retardar a convergência ${ }^{[11]}$.

O método SLOR (sucessive line over-relaxation), nada mais que é o método SOR só que aplicado em linhas ou colunas. Cada linha ou coluna é resolvida usando um método direto. Será utilizado o algoritmo de Thomas ${ }^{[1}$. para resolver as equações em cada linha.

\subsection{Caso Bidimensional Estacionário}

\subsubsection{Equações Governantes}

Aqui são tratadas as equações TSD no caso bidimensional e estacionário, ou seja, a equação governante a ser tratada aqui provêm da equação (2.65) no caso bidimensional, isto é:

$$
\left(E+2 F \phi_{x}\right) \phi_{x x}+\phi_{y y}=0
$$

onde

$$
\begin{gathered}
E=1-M_{\infty}^{2} ; \\
F=-\frac{1}{2}[\gamma+1] M_{\infty}^{2} .
\end{gathered}
$$

As coordenadas $x$ e $y$ são usadas para definir o plano da seção de aerofólio ao invés de $x$ e $z$ do caso tridimensional. Além disso, $\phi^{s}$ passa a ser denotado por $\phi$.

\subsubsection{Detector de Regime Subsônico/Sônico/Supersônico}

Observe que a equação TSD, (2.1), no caso bidimensional estacionário é dado da seguinte forma:

$$
\left(a^{2}-U^{2}\right) \phi_{x x}-2 U V \phi_{x y}+\left(a^{2}-V^{2}\right) \phi_{y y}=0
$$


As equações diferenciais parciais de $2^{a}$ de segunda ordem

$$
A \phi_{x x}+B \phi_{x y}+C \phi_{y y}+D \phi_{x}+E \phi_{y}+F \phi=0
$$

são classificadas da seguinte maneira ${ }^{[11]}$ :

- elíptica: se $B^{2}-4 A C<0$;

- parabólica: se $B^{2}-4 A C=0$;

- hiperbólica: se $B^{2}-4 A C>0$.

Analisando a equação (2.79), tem-se que:

$$
B^{2}-4 A C=4 U^{2} V^{2}-4\left(a^{2}-U^{2}\right)\left(b^{2}-V^{2}\right)=-4 a^{2}\left[a^{2}-\left(U^{2}+V^{2}\right)\right]
$$

assim:

- elíptica: se $\sqrt{U^{2}+V^{2}}<a$, isto é, subsônica;

- parabólica: se $\sqrt{U^{2}+V^{2}}=a$, isto é, sônica;

- hiperbólica: se $\sqrt{U^{2}+V^{2}}>a$, isto é, supersônica.

Aplicando os critérios acima para definir se o ponto a ser analisado é subsônico, sônico ou supersônico na equação (2.78), obtém-se um detector de condição subsônica, sônica ou supersônica, dado por:

- subsônica: se $\left(E+2 F \phi_{x}\right)<0$;

- sônica: se $\left(E+2 F \phi_{x}\right)=0$;

- supersônica: se $\left(E+2 F \phi_{x}\right)>0$.

Este detector é necessário para a escolha do tipo de formulação de diferenças finitas a ser utilizado nas derivadas. 


\subsubsection{Método de discretização}

Se o escoamento é subsônico no ponto a ser analisado, as formulações de diferenças finitas para as derivadas de primeira ordem serão:

$$
\begin{aligned}
& \phi_{x}=\frac{1}{2}\left(\frac{\phi_{i+1 j}-\phi_{i j}}{x_{i+1}-x_{i}}+\frac{\phi_{i j}-\phi_{i-1 j}}{x_{i}-x_{i-1}}\right) ; \\
& \phi_{y}=\frac{1}{2}\left(\frac{\phi_{i j+1}-\phi_{i j}}{y_{j+1}-y_{j}}+\frac{\phi_{i j}-\phi_{i j-1}}{y_{j}-y_{j-1}}\right) ;
\end{aligned}
$$

os sub-índices $i, j$ identificam um ponto na $i, j$-ésima posição nos eixos $x, y$ respectivamente na malha bidimensional. As derivadas de segunda ordem, são dadas por ${ }^{[3]}$ :

$$
\begin{gathered}
\phi_{x x}=\frac{2}{x_{i+1}-x_{i-1}}\left(\frac{\phi_{i+1 j}-\phi_{i j}}{x_{i+1}-x_{i}}-\frac{\phi_{i j}-\phi_{i-1 j}}{x_{i}-x_{i-1}}\right) ; \\
\phi_{y y}=\frac{2}{y_{j+1}-y_{j-1}}\left(\frac{\phi_{i j+1}-\phi_{i j}}{y_{j+1}-y_{j}}-\frac{\phi_{i j}-\phi_{i j-1}}{y_{j}-y_{j-1}}\right) .
\end{gathered}
$$

No caso do escoamento ser supersônico no ponto a ser analisado, as modificações serão nas formulações de diferenças finitas para as derivadas $\phi_{x}, \phi_{x x}$, as que têm dependência no eixo $x$, que são dadas por

$$
\begin{gathered}
\phi_{x}=\frac{\phi_{i j}-\phi_{i-1 j}}{x_{i}-x_{i-1}} ; \\
\phi_{x x}=\frac{1}{x_{i}-x_{i-1}}\left(\frac{\phi_{i j}-\phi_{i-1 j}}{x_{i}-x_{i-1}}-\frac{\phi_{i-1 j}-\phi_{i-2 j}}{x_{i-1}-x_{i-2}}\right) .
\end{gathered}
$$

Estas formulações serão aplicadas na equação (2.78) sendo que será necessário um sistema que mude o esquema de diferenciação finita para cada caso. Para isto, será usado o detector descrito na subseção anterior, 2.5.2. Lembrando a equação (2.9), define-se:

$$
\begin{gathered}
\xi_{i, j}^{c}=\left(1-M_{\infty}^{2}\right)-(\gamma+1) M_{\infty}^{2}\left[\frac{1}{2}\left(\frac{\phi_{i+1 j}-\phi_{i j}}{x_{i+1}-x_{i}}+\frac{\phi_{i j}-\phi_{i-1 j}}{x_{i}-x_{i-1}}\right)\right] ; \\
\xi_{i j}^{a}=\left(1-M_{\infty}^{2}\right)-(\gamma+1) M_{\infty}^{2}\left(\frac{\phi_{i j}-\phi_{i-1 j}}{x_{i}-x_{i-1}}\right)
\end{gathered}
$$

$\mathrm{e}$

$$
\begin{gathered}
\mu_{i j}^{c}=\left\{\begin{array}{l}
0, \text { se } \xi_{i, j}^{c}>0 \\
1, \text { se } \xi_{i, j}^{c} \leq 0
\end{array} ;\right. \\
\mu_{i j}^{a}=\left\{\begin{array}{l}
0, \text { se } \xi_{i, j}^{a}>0 \\
1, \text { se } \xi_{i, j}^{a} \leq 0
\end{array}\right.
\end{gathered}
$$


Como se está utilizando as diferenças centrais e atrasadas, então define-se $\phi_{x x}^{c}$ igual a $\phi_{x x}$ da equação (2.84) e $\phi_{x x}^{a}$ igual a $\phi_{x x}$ da equação (2.87). Implementando o sistema de detecção de escoamento sônico, subsônico ou supersônico a condição de choque se torna mais precisa com a seguinte formulação ${ }^{[25]}$ :

$$
\xi_{i, j}^{c} \phi_{x x}^{c}+\phi_{y y}-\mu_{i, j}^{c} \xi_{i, j}^{c} \phi_{x x}^{c}+\mu_{i, j}^{a} \xi_{i, j}^{a} \phi_{x x}^{a}=0
$$

fixando os $\xi_{i, j}^{c}, \xi_{i, j}^{a}$ como constantes, atualizados a cada iteração com os valores da iteração anterior. Ao discretizarmos em diferenças finitas obtemos:

$$
\begin{aligned}
& \left(\frac{2}{y_{j+1}-y_{j-1}}\right)\left(\frac{1}{y_{j}-y_{j-1}}\right) \phi_{i j-1}^{+} \\
+ & {\left[\left(\frac{2}{y_{j+1}-y_{j-1}}\right)\left(-\frac{1}{y_{j+1}-y_{j}}-\frac{1}{y_{j}-y_{j-1}}\right)\right.} \\
& \left.+\xi_{i, j}^{c}\left(\frac{2}{x_{i+1}-x_{i-1}}\right)\left(1-\mu_{i, j}^{c}\right)+\mu_{i, j}^{a} \xi_{i, j}^{a}\left(\frac{1}{x_{i}-x_{i-1}}\right)\right] \phi_{i, j}^{+} \\
+ & \left(\frac{2}{y_{j+1}-y_{j-1}}\right)\left(\frac{1}{y_{j+1}-y_{j}}\right) \phi_{i j+1}^{+} \\
= & -\xi_{i, j}^{c}\left(\frac{2}{x_{i+1}-x_{i-1}}\right)\left(\frac{\phi_{i+1}}{x_{i+1}-x_{i}}+\frac{\phi_{i-1 j}}{x_{i}-x_{i-1}}\right) \\
& +\mu_{i, j}^{a} \xi_{i, j}^{a}\left(\frac{1}{x_{i}-x_{i-1}}\right)\left(\frac{-\phi_{i-1 j}}{x_{i}-x_{i-1}}-\frac{\phi_{i-1 j}-\phi_{i-2 j}}{x_{i-1}-x_{i-2}}\right) .
\end{aligned}
$$

O sobrescrito “+” denota que o valor do potencial é da iteração atual, ou seja, é desconhecido e terá que ser resolvido. Os potenciais sem o sobrescrito "+" assumirão valores da iteração anterior.

\section{Correção de Entropia}

No caso de correção de entropia (lembrando da equação (2.23)), os termos $\xi_{i j}^{c}$ e $\xi_{i j}^{a}$ da equação (2.93) serão substituídos por:

$$
\begin{aligned}
\xi_{i j}^{c}=(\gamma+1) M_{\infty}^{2} R & {\left[\tilde{V}\left(\frac{1+R}{1+R+\frac{1}{2}\left(\frac{\phi_{i+1 j}-\phi_{i j}}{x_{i+1}-x_{i}}+\frac{\phi_{i j}-\phi_{i-1 j}}{x_{i}-x_{i-1}}\right)}\right)^{2}\right.} \\
& \left.-V\left(\frac{1+R}{1+R+\frac{1}{2}\left(\frac{\phi_{i+1 j}-\phi_{i j}}{x_{i+1}-x_{i}}+\frac{\phi_{i j}-\phi_{i-1 j}}{x_{i}-x_{i-1}}\right)}\right)^{2}\right]
\end{aligned}
$$




$$
\begin{aligned}
\xi_{i, j}^{a}=(\gamma+1) M_{\infty}^{2} R & {\left[\tilde{V}\left(\frac{1+R}{1+R+\left(\frac{\phi_{i j}-\phi_{i-1 j}}{x_{i}-x_{i-1}}\right)}\right)^{2}\right.} \\
& \left.-V\left(\frac{1+R}{1+R+\left(\frac{\phi_{i j}-\phi_{i-1 j}}{x_{i}-x_{i-1}}\right)}\right)^{2}\right] .
\end{aligned}
$$

\section{Correção de Entropia e Vorticidade}

E no caso de correção de entropia e vorticidade, os termos $\xi_{i, j}^{c}$ e $\xi_{i, j}^{a}$ da equação (2.93) serão substituídos por:

$$
\xi_{i j}^{c}=(\gamma+1) M_{\infty}^{2} R\left[\tilde{V}\left(\frac{1+R}{1+R+\phi_{x}^{c}}\right)^{2}-V\left(\frac{1+R}{1+R+\phi_{x}^{c}}\right)^{2}\right]
$$

onde $\phi_{x}^{c}=\frac{1}{2}\left(\frac{\phi_{i+1 j}-\phi_{i j}}{x_{i+1}-x_{i}}+\frac{\phi_{i j}-\phi_{i-1 j}}{x_{i}-x_{i-1}}\right)-\frac{1}{\gamma(\gamma-1) M_{\infty}^{2}} \frac{s}{c_{v}}, \mathrm{e}$

$$
\xi_{i, j}^{a}=(\gamma+1) M_{\infty}^{2} R\left[\tilde{V}\left(\frac{1+R}{1+R+\phi_{x}^{a}}\right)^{2}-V\left(\frac{1+R}{1+R+\phi_{x}^{a}}\right)^{2}\right] .
$$

$\operatorname{com} \phi_{x}^{a}=\left(\frac{\phi_{i j}-\phi_{i-1 j}}{x_{i}-x_{i-1}}\right)-\frac{1}{\gamma(\gamma-1) M_{\infty}^{2}} \frac{s}{c_{v}}$.

\section{Correção de Viscosidade}

A interação entre a onda de choque e a camada limite é analisada resolvendo as equações para o escoamento invíscido e usando a solução da distribuição de velocidades sobre a superfície de sustentação para resolver as equações da camada limite. A solução da camada limite, na forma de uma espessura de deslocamento, é então utilizada para a obtenção de uma nova solução invíscida. O processo é repetido até que se obtenha convergência nas duas soluções. O método de correção viscosa investigado nesta etapa do projeto de pesquisa envolve a solução das equações da camada limite através de um método de integração numérica

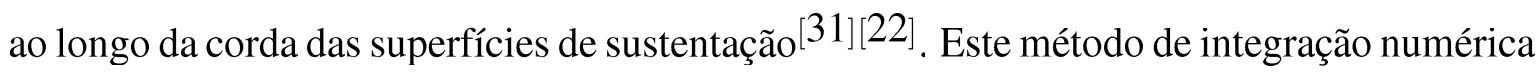
foi escolhido devido à boa correlação dos resultados numéricos com correção viscosa com os dados experimentais apresentados na Referência [22].

Assume-se aqui que em escoamento transônico as regiões laminar e de transição da camada limite são muito pequenas quando comparadas com a região turbulenta. No presente estudo as regiões laminar e de transição são desprezadas assumindo que o primeiro ponto 
da malha, a jusante do bordo de ataque, já é turbulento. As equações da continuidade e quantidade de movimento para uma camada limite bidimensional, compressível e turbulenta são[22]:

$$
\left\{\begin{array}{l}
\frac{\partial \rho u}{\partial s}+\frac{\partial \rho w}{\partial n}=0 \\
\rho u \frac{\partial u}{\partial s}+\rho w \frac{\partial u}{\partial n}=\frac{\partial p}{\partial s}+\frac{\partial \tau}{\partial n}
\end{array}\right.
$$

onde $p$ é a pressão, $\rho$ é a densidade, $\tau$ é a tensão de cisalhamento, $u$ e $w$ são as componentes de velocidade média nas direções do escoamento, $s$, e normal, $n$. Utiliza-se a transformação de coordenadas de Mager ${ }^{[22}$

$$
\left\{\begin{array}{l}
\hat{s}=\int_{0}^{s}\left(\frac{T_{0}}{T}\right)\left(\frac{T_{e}}{T_{0}}\right)^{\frac{\gamma+1}{2(\gamma-1)}} d s \\
\hat{n}=\sqrt{\frac{T_{e}}{T_{0}}} \int_{0}^{n}\left(\frac{\rho}{\rho_{0}}\right) d n
\end{array}\right.
$$

onde $T$ é a temperatura de referência e os subscritos " $e$ "e " 0 "denotam a fronteira da camada limite e a condição de estagnação, respectivamente. Para o ar, em condições típicas de vôo transônico, têm-se que a razão do calor específico $\gamma$ é igual a 1,4. A temperatura de referência $T$ é expressa como uma função do número de Prandtl, Pr,

$$
\frac{T}{T_{0}}=0,5 g+0,22 \sqrt[3]{\operatorname{Pr}}+(0,5-0,22 \sqrt[3]{\operatorname{Pr}})\left(\frac{T_{e}}{T_{0}}\right)
$$

onde $g$ é igual a 1 para escoamentos adiabáticos e $\operatorname{Pr}=0,72$ para o ar em condições típicas de vôo transônico. Aplicando a transformação da Eq.(2.99) na Eq.(2.98) e reescrevendo em função da espessura de momentum transformada, $\hat{\theta}$, e fator de forma incompressível transformado, $\hat{H}_{i}$, obtêm-se

$$
\left\{\begin{aligned}
\frac{d \hat{\theta}}{d \hat{s}}= & -\frac{\hat{\theta}}{\hat{U}_{e}} \frac{d \hat{U}_{e}}{d \hat{s}}\left(2+\hat{H}_{i}\right)+\left(\frac{T_{0}}{T_{e}}\right)^{0.268}\left(\frac{T}{T_{0}}\right)^{1.268} \frac{\tau_{w}}{\rho_{e} \hat{U}_{e}^{2}} \\
\frac{d \hat{H}_{i}}{d \hat{s}}= & -\frac{1}{2 \hat{U}_{e}} \frac{d \hat{U}_{e}}{d \hat{s}}\left(\hat{H}_{i}+1\right)^{2}\left(\hat{H}_{i}-1\right) \hat{H}_{i}+ \\
& \frac{\hat{H}_{i}^{2}-1}{\hat{\theta}}\left(\frac{T}{T_{e}}\right) \frac{\tau_{w}}{\rho_{e} \hat{U}_{e}^{2}}\left[\hat{H}_{i}+\left(\hat{H}_{i}+1\right) \int_{0}^{1} \frac{\tau}{\tau_{w}} d\left(\frac{\hat{n}}{\hat{\delta}}\right)\right]
\end{aligned}\right.
$$

onde o subscrito $w$ denota a condição na parede, $\hat{U}$ é a componente de velocidade na direção $x$ nas coordenadas transformadas, $\tau$ é a tensão de cizalhamento na parede, e $\hat{\delta}$ é a espesura da camada limite nas coordenadas transformadas. É possível reescrever esta equação usando 
o número de Mach na fronteira da camada limite através das relações

$$
\left\{\begin{array}{l}
M_{e}=\frac{u_{e}}{a_{e}}=\frac{u_{e}}{a_{0}} \frac{a_{0}}{a_{e}}=\frac{u_{e}}{a_{0}} \sqrt{\frac{T_{0}}{T_{e}}} \\
u_{e}=\hat{U}_{e} \sqrt{\frac{T_{e}}{T_{0}}}
\end{array}\right.
$$

e usando a aproximação

$$
\int_{0}^{1} \frac{\tau}{\tau_{w}} d\left(\frac{\hat{n}}{\hat{\delta}}\right) \approx 0,011\left(\frac{\hat{H}_{i}-1}{\hat{H}_{i}}\right)^{2} \frac{2}{C_{f}}
$$

sendo que

$$
\frac{T_{0}}{T_{e}}=1+\frac{\gamma-1}{2} M_{e}^{2}
$$

O coeficiente de atrito, $C_{f}$, é dado por ${ }^{[31]}$

$$
C_{f}=2 \frac{\tau_{w}}{\rho_{e} \hat{U}_{e}^{2}}
$$

que, no caso incompressível, é aproximado por

$$
C_{f}=0,246 e^{-1.561 \hat{H}_{i}}\left(\frac{u_{e} \theta}{\bar{v}}\right)^{-0,268}\left(\frac{T_{e}}{T}\right)^{1,268} ;
$$

onde $\bar{v}$ é a viscosidade cinemática. Como

$$
\theta=\left(\frac{T_{0}}{T_{e}}\right)^{3} \hat{\theta}
$$

temos que, ao substituir as equações (2.102) e (2.107) em (2.106), tem-se

$$
C_{f}=0,246 e^{-1,561 \hat{H}_{i}}\left(\frac{T_{0}}{T_{e}}\right)^{-1,804}\left(\frac{T}{T_{0}}\right)^{-1,268}\left(\frac{M_{e} \hat{\theta} a_{0}}{\bar{v}}\right)^{-0,268} .
$$

Da equação (2.102) temos que

$$
\left\{\begin{array}{l}
M_{e}=\frac{\hat{U}_{e}}{a_{0}} \frac{T_{0}}{T_{e}} \\
\frac{1}{\hat{U}_{e}}=\frac{1}{a_{0}} \frac{T_{0}}{T_{e}} \frac{1}{M_{e}}
\end{array} \Rightarrow-\frac{\hat{\theta}}{\hat{U}_{e}} \frac{d \hat{U}_{e}}{d \hat{s}}=-\frac{\hat{\theta}}{M_{e}} \frac{d M_{e}}{d \hat{s}}\right.
$$

Aplicando as equações (2.103), (2.105), (2.107) e (2.109) na equação (2.101) obtêm-se então

$$
\left\{\begin{aligned}
\frac{d \hat{\theta}}{d \hat{s}}= & -\frac{\hat{\theta}}{M_{e}} \frac{d M_{e}}{d \hat{s}}\left(2+\hat{H}_{i}\right)+\left(\frac{T_{0}}{T_{e}}\right)^{0,268}\left(\frac{T_{r e f}}{T_{0}}\right)^{1,268} \frac{C_{f}}{2} \\
\frac{d \hat{H}_{i}}{d \hat{s}}= & \frac{\hat{H}_{i}^{2}-1}{\hat{\theta}}\left(\frac{T}{T_{0}}\right)\left[\frac{C_{f}}{2} \hat{H}_{i}+0,011\left(\hat{H}_{i}+1\right)\left(\frac{\hat{H}_{i}-1}{\hat{H}_{i}}\right)^{2}\right]- \\
& \frac{\left(\hat{H}_{i}+1\right)^{2}\left(\hat{H}_{i}-1\right) \hat{H}_{i}}{2 M_{e}} \frac{d M_{e}}{d \hat{s}}
\end{aligned}\right.
$$


As Eqs.(2.110) podem ser integradas em $\hat{s}$ usando um método Runge-Kutta de quarta ordem, por exemplo. As condições iniciais utilizadas para o ponto mais próximo do bordo de ataque foram $\hat{\theta}=10^{-5}$ e $\hat{H}_{i}=1$ (seguindo a sugestão dada pela referência [22]). A espessura de deslocamento, $\delta^{*}$, é calculada usando

$$
\delta^{*}=\left(\hat{\boldsymbol{\theta}}+\hat{\boldsymbol{\delta}}^{*}\right)\left(\frac{T_{0}}{T_{e}}\right)^{\frac{3 \gamma-1}{2(\gamma-1)}}-\hat{\boldsymbol{\theta}}\left(\frac{T_{0}}{T_{e}}\right)^{\frac{\gamma+1}{2(\gamma-1)}}
$$

$\mathrm{e}$

$$
\hat{\delta}^{*}=\hat{\theta} \hat{H}_{i}
$$

A espessura de deslocamento, $\delta^{*}$, é usada então para modificar a forma da superfície de sustentação na solução do escoamento invíscido.

\subsubsection{Método Numérico}

A característica geral dos métodos iterativos convergentes é que, para casos estacionários, conforme as iterações progridem a diferença sucessiva entre as aproximações $\phi^{(n+1)}$ e $\phi^{(n)}$ diminui. Aqui o sobrescrito indica o número da iteração. Porém, para obter uma boa solução, para o sistema de equações em estudo, é necessário um número muito grande de iterações. Para amenizar este problema aumentamos deliberadamente essa pequena diferença, extrapolando (sobre-relaxando) o valor de $\phi^{(n+1)}$, de forma que se aproxime mais rapidamente da solução numérica $\phi$ do sistema de equações. Essa é a base dos métodos tipo SOR(Sucessive Over-Relaxation).

O método SLOR (sucessive line over-relaxation), nada mais que é o método SOR só que aplicado em linhas ou colunas. Este é o método utilizado no presente estudo. Cada linha é resolvida, de forma direta, usando o algoritmo de Thomas ${ }^{[2]}$. O algoritmo pode ser resumido como

$$
\phi_{\text {SLOR }}^{(n+1)}=\phi^{(n)}+(\omega-1)\left(\phi^{(n)}-\phi_{\text {SOR+Thomas }}^{(n+1)}\right) .
$$

onde $\omega$ é o fator de relaxação.

\subsubsection{Esquematização da Malha}

Seja a malha na qual será feita a simulação dada conforme a Figura 2.4, onde 


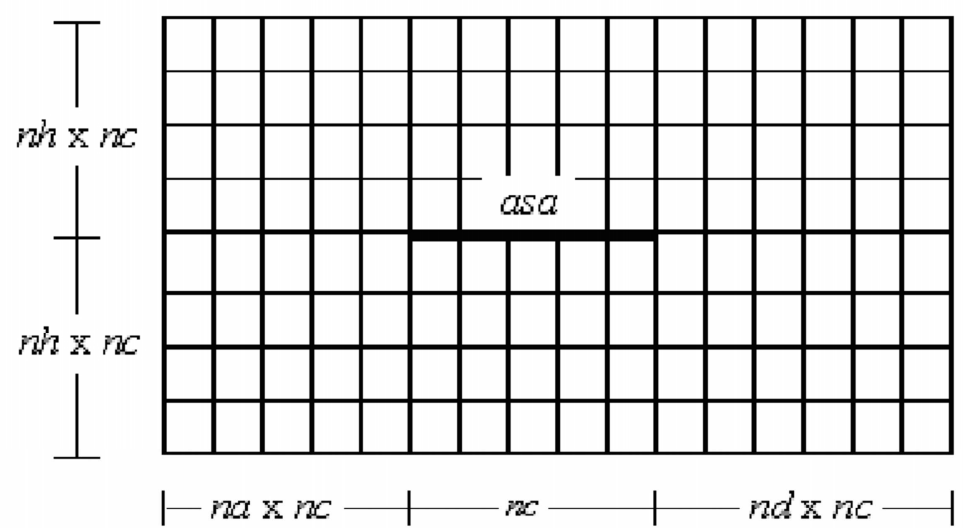

Figura 2.4: Definições para a malha computacional.

$n c=$ número de pontos na corda;

$n a=$ número de vezes de corda antes do bordo de ataque;

$n d=$ número de vezes de corda depois do bordo de fuga;

$n h=$ número de vezes de corda acima/abaixo da asa;

e seja

$m=$ número de pontos na coordenada $x$

$n=\mathrm{o}$ número de pontos na coordenada $y$.

Com a utilização da teoria de pequenas perturbações pode-se aplicar a condição de contorno do perfil da asa em um segmento de reta na malha. Esta representação da asa permite usar uma malha cartesiana levando a um custo computacional bem mais baixo que aquele associado com outros métodos (exemplo, utilização de malhas não estruturadas), além de ser bem mais simples de se implementar.

A malha que será utilizada para simular os casos é uma malha estruturada cartesiana não uniforme com distribuição de pontos obedecendo uma função de classe $C_{\infty}$ (diferenciável em todas as ordens), para obter uma distribuição de pontos suave. Um exemplo de malha é mostrada na Figura 2.5.

Um fator importante na formulação da malha, é que a linha central (na horizontal) contém 2 linhas contendo o extradorso e intradorso da seção de aerofólio. 


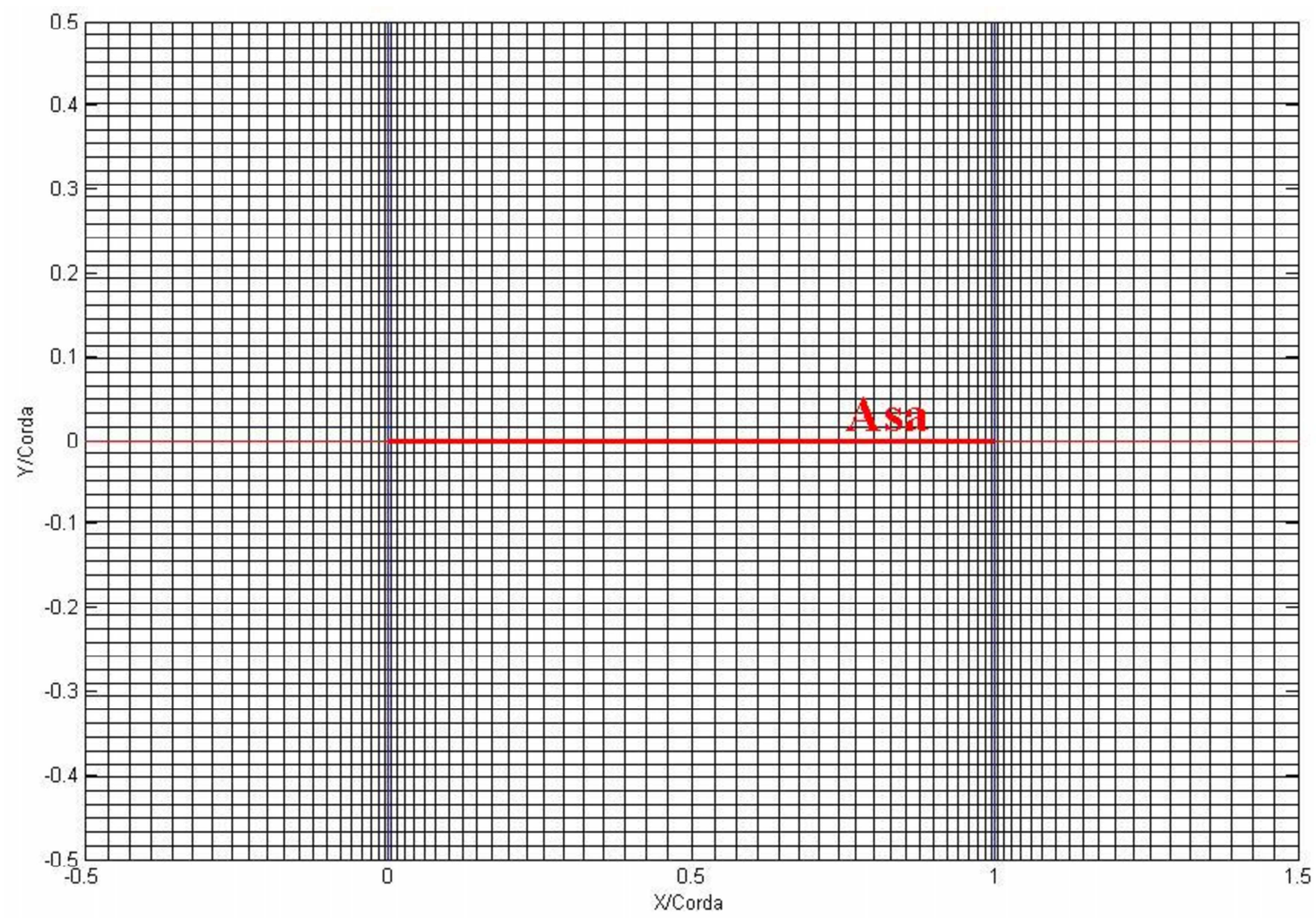

Figura 2.5: Exemplo de malha utilizada nas simulações (esta é uma visão ampliada em torno da asa).

\subsubsection{Condições de Contorno}

Serão utilizados três tipos de condições nas linhas que contém a seção de aerofólio, referentes às coordenadas $j=n h \times n c$ e $j=n h \times n c+1$. As condições usadas nessas linhas são:

- A linha inferior, $j=n h \times n c$, a montante do bordo de ataque, assumirá a condição de continuidade da solução

$$
\phi_{i n h \times n c}=\phi_{i n h \times n c+1} \text {; }
$$

- Na superfície da seção de aerofólio a condição é que a velocidade normal à superfície é nula, isto é

$$
V_{\text {normal }}=\operatorname{sen} \theta u+\cos \theta v=0
$$

onde $\theta$ é o ângulo de inclinação da superfície.

- A jusante do bordo de fuga, as componentes de velocidades na linha $j=n h \times n c$ são dadas por $u_{i n h \times n c}=u_{i n h \times n c+1}$ e na linha $j=n h \times n c+1$ é $v_{i n h \times n c}=v_{i n h \times n c+1}$. 
Agora as condições de contorno na fronteira da região de domínio a ser simulado são:

- Na fronteira lateral esquerda, entrada do escoamento, temos $\phi_{1 j}=\phi_{2 j}$ (velocidade uniforme);

- Na fronteira lateral direita, saída do escoamento, temos $\phi_{m j}=\phi_{m-1 j}$ (velocidade uniforme);

- Na fronteira inferior temos $\phi_{i 1}=\phi_{i 2}$ (velocidade normal é nula);

- Na fronteira superior temos $\phi_{i n}=\phi_{i n-1}$ (velocidade normal é nula).

Pode-se observar melhor estas codições na Figura 2.6:

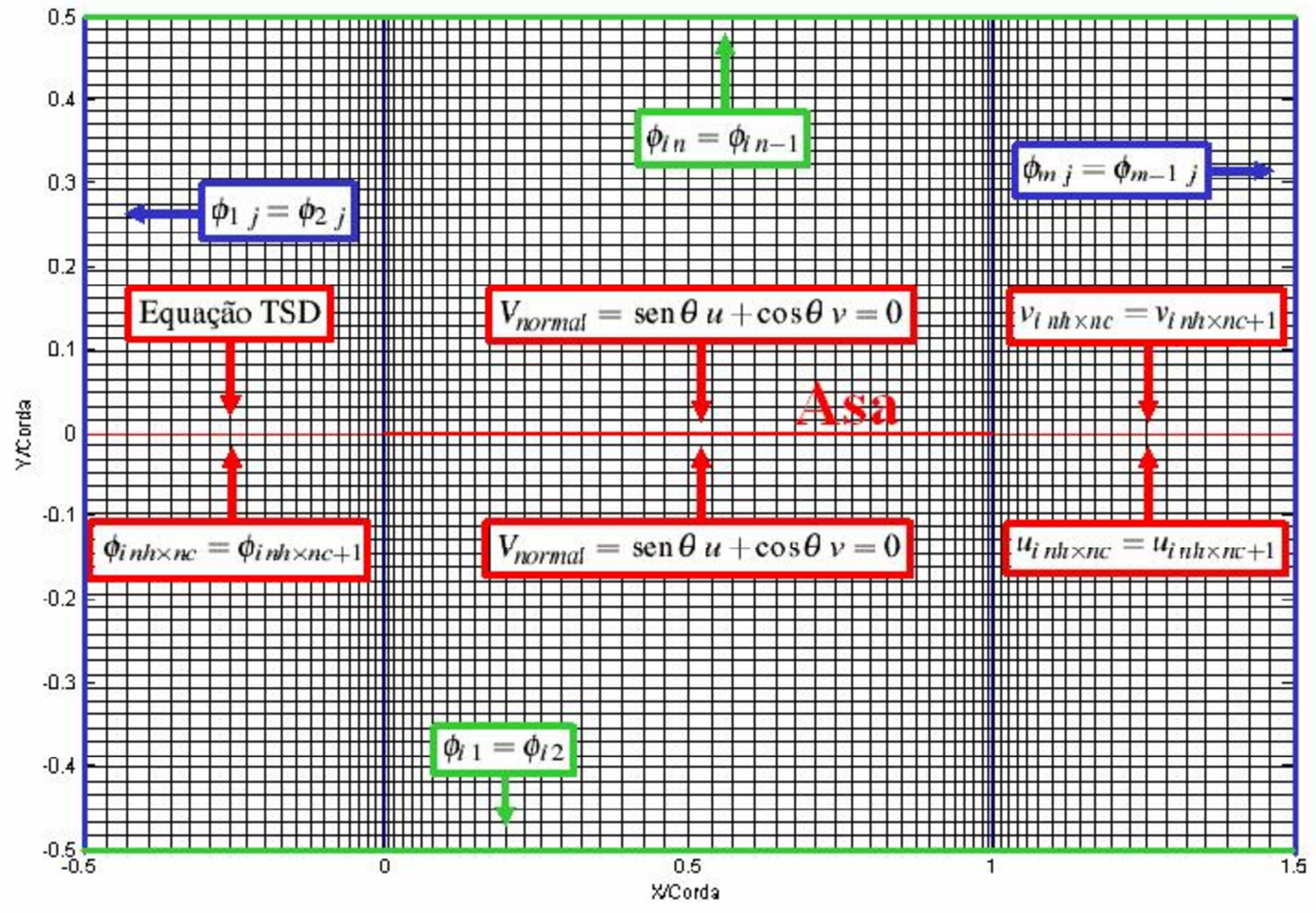

Figura 2.6: Condições de contorno na malha. 


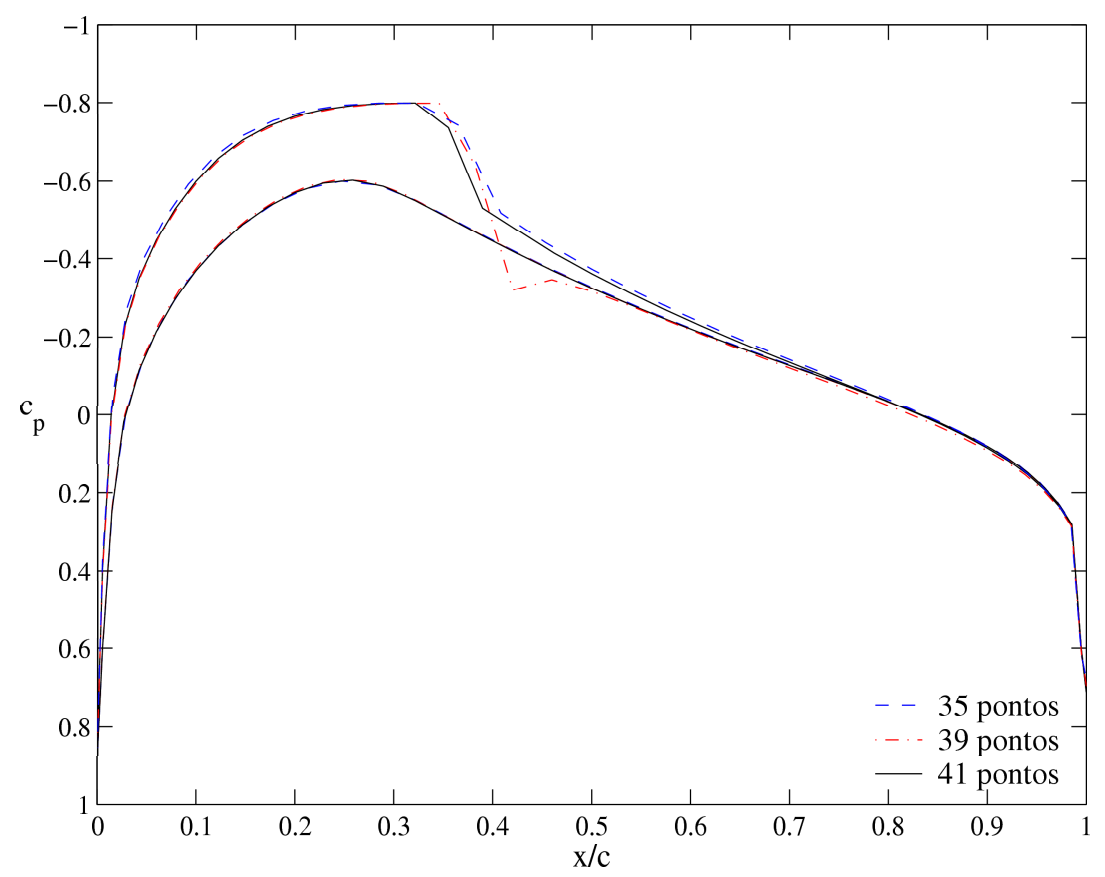

Figura 2.7: Distribuição do coeficiente de pressão para o perfil NACA0012 em $M_{\infty}=0,750$ e $\alpha=1,00^{\circ}$, com os segintes refinamentos na corda: 35 pontos, 39 pontos e 41 pontos

\subsubsection{Refinamento e Dimensão da Malha}

\section{Refinamento}

A escolha do refinamento mais adequado para o caso bidimensional foi através de alguns testes numéricos, fixando como referêncial o número de pontos sob o perfil. Os testes foram feitos com os seguintes números de pontos sob o perfil: $35,39,41,45,51$.

Pode-se observar na Figura 2.7, a distribuição do coeficiente de pressão para o perfil NACA0012 em $M_{\infty}=0,750$ e $\alpha=1,00^{\circ}$, até um refinamento de 41 pontos sob uma corda há uma grande variação na distribuição do coeficiente de pessão, e a partir de 41 pontos em diante a variação da distribuição do coeficiente de pressão é muito pequena, como mostra a Figura 2.8, e o custo computacional aumenta drasticamente a cada ponto adicionado no refinamento.

A mesma análise foi feita para o perfil NACA0012 em $M_{\infty}=0,800$ e $\alpha=1,25^{\circ}$ e observou-se o mesmo comportamento, como mostram as figuras 2.9 e 2.10 . 


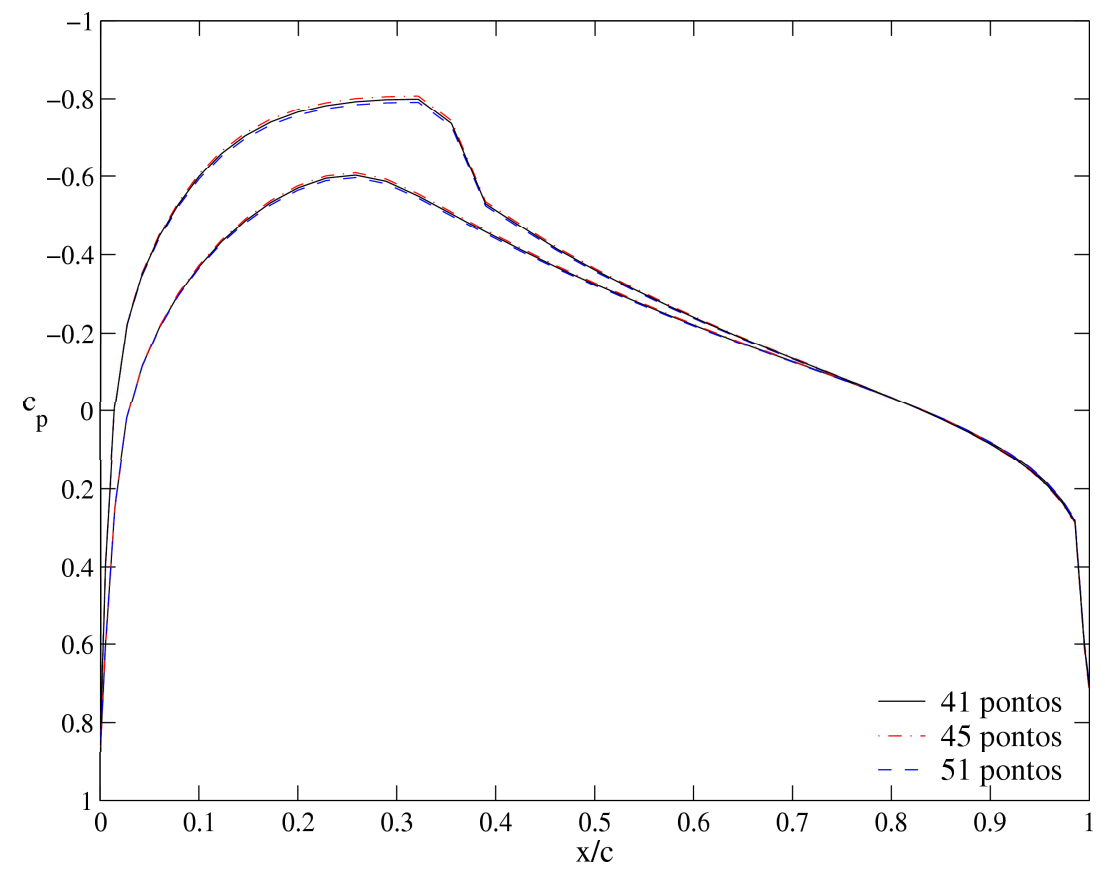

Figura 2.8: Distribuição do coeficiente de pressão para o perfil NACA0012 em $M_{\infty}=0,750$ e $\alpha=1,00^{\circ}$, com os segintes refinamentos na corda: 41 pontos, 45 pontos e 51 pontos

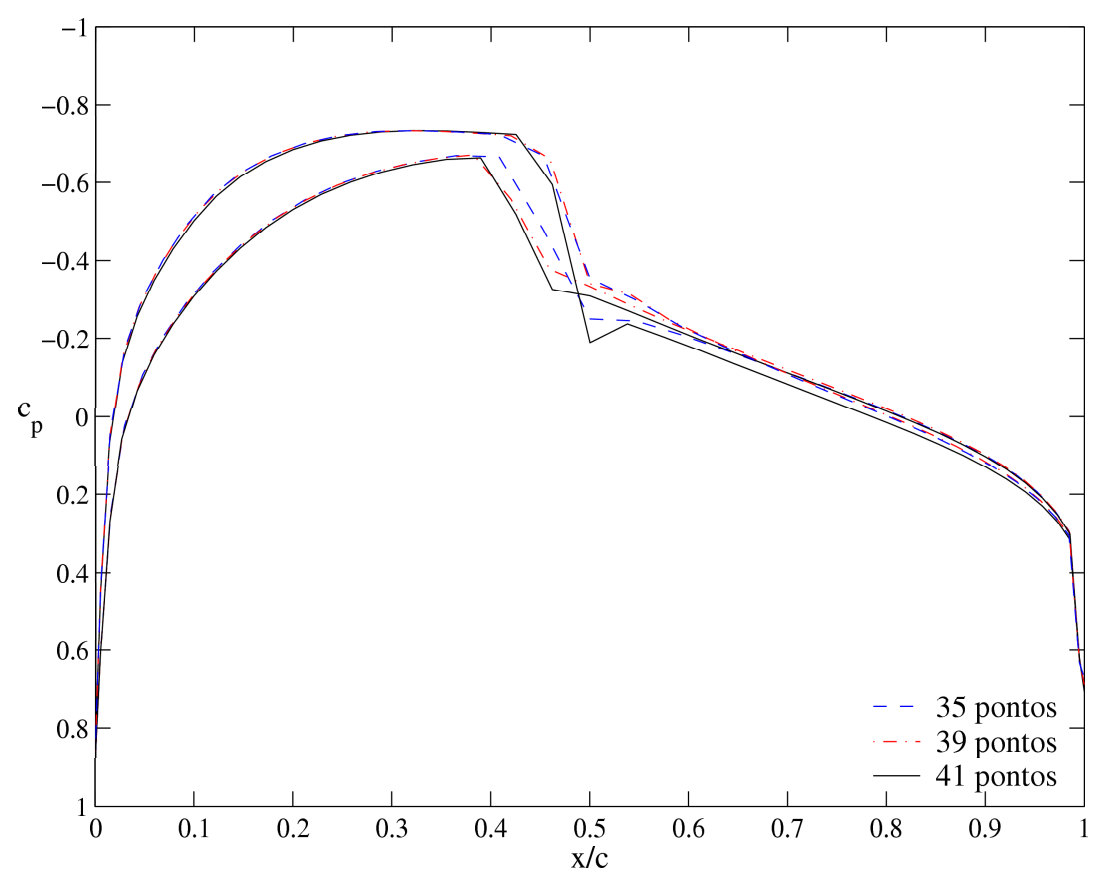

Figura 2.9: Distribuição do coeficiente de pressão para o perfil NACA0012 em $M_{\infty}=0,800$ e $\alpha=1,25^{\circ}$, com os segintes refinamentos na corda: 35 pontos, 39 pontos e 41 pontos 


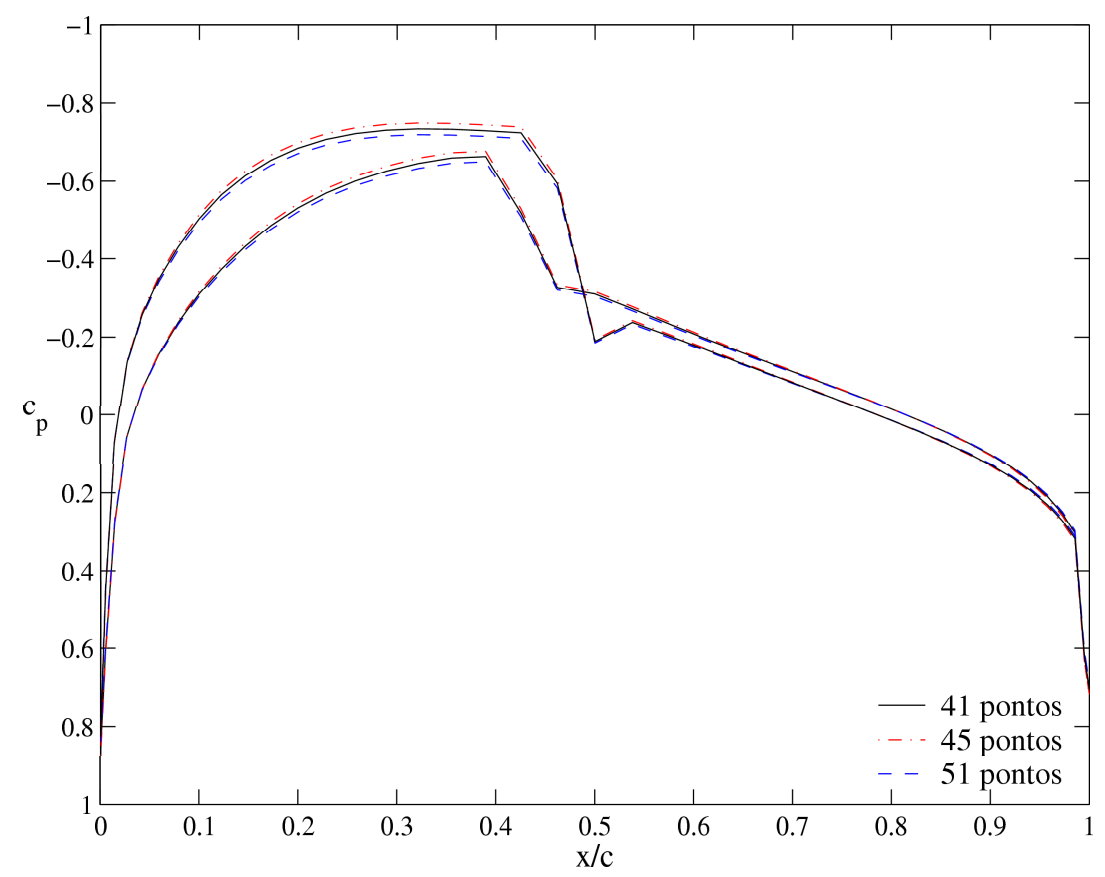

Figura 2.10: Distribuição do coeficiente de pressão para o perfil NACA0012 em $M_{\infty}=0,800$ e $\alpha=1,25^{\circ}$, com os segintes refinamentos na corda: 41 pontos, 45 pontos e 51 pontos

Devido à pequena varição na distribuição de pressão com um refinamento a partir de 41 pontos sob uma corda, os resultados obtidos no Capítulo 3 foram com um refinamento de 41 pontos sob uma corda.

\section{Dimensão da Malha}

Seguindo as notações da Seção 2.5.5, foi feita uma análise no tamanho da malha mais adequada para simular os resultados do Capítulo 3. Observou-se inicialmente que a distância da fronteira superior e inferior afetava os resultados, quanto menor a distância, ocorria um fenômeno de influência das fronteiras superior e inferior, como pode-se ver na figura 2.11. A partir de 3 cordas de distância, o resultado estabiliza, como pode-se observar na figura 2.12.

Quanto a distância antes do bordo de ataque e bordo de fuga, obsevou-se que a partir de uma corda de distância a influência das fronteiras a montante do bordo de ataque e a jusante do bordo de fuga são nulas, como pode-se observar na figura 2.13.

Portanto a dimensão utilizada no programa bidimensional a malha utilizada é de $n c=41$, $n a=1, n d=1$ e $n h=3$. 


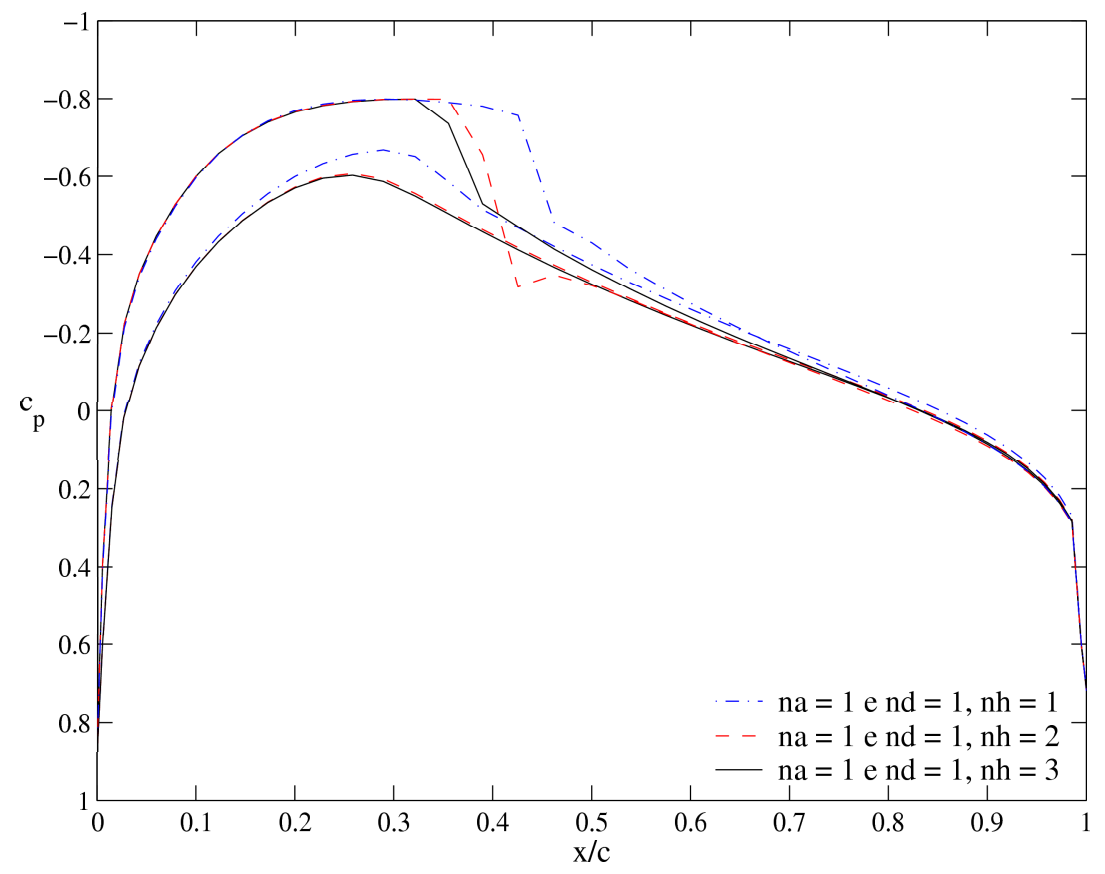

Figura 2.11: Distribuição do coeficiente de pressão para o perfil NACA0012 em $M_{\infty}=0,750$ e $\alpha=1,00^{\circ}$, com a distância da frontria superior e infreior, $n h$, variando em 1,2 e 3 cordas.

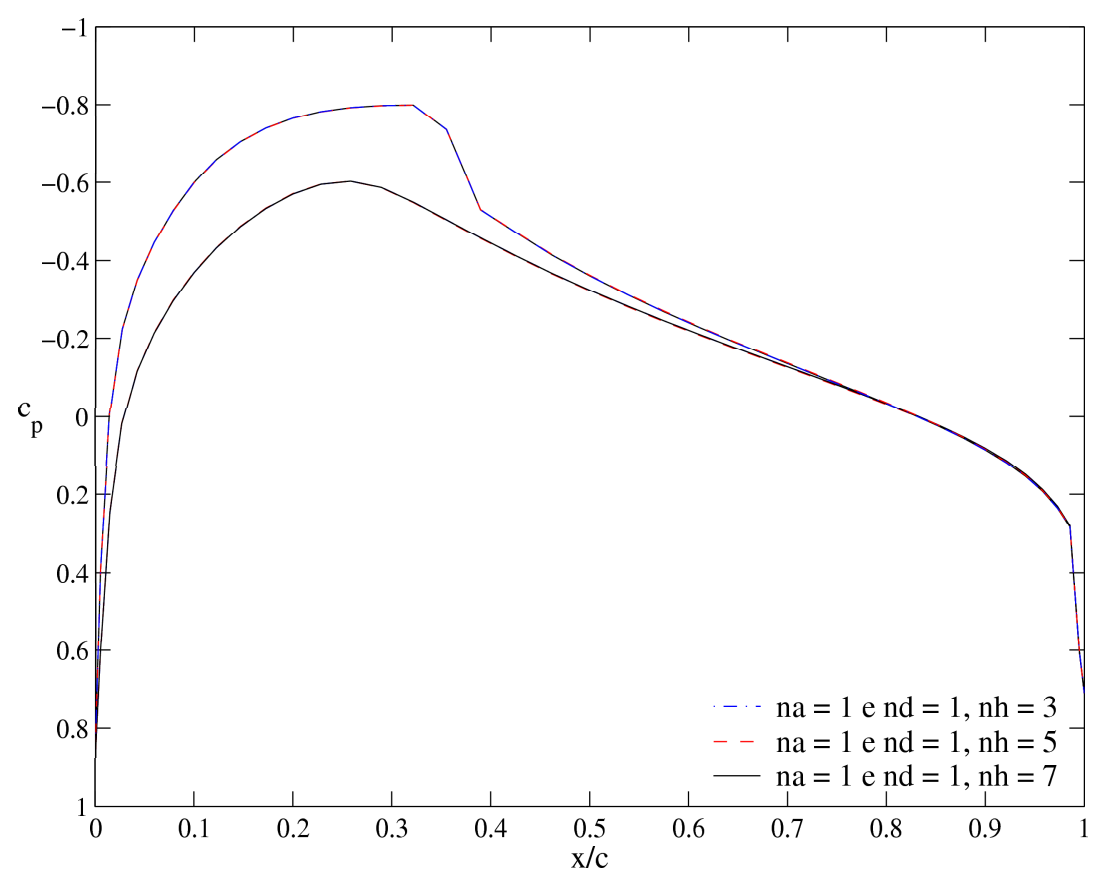

Figura 2.12: Distribuição do coeficiente de pressão para o perfil NACA0012 em $M_{\infty}=0,750$ e $\alpha=1,00^{\circ}$, com a distância da frontria superior e infreior, $n h$, variando em 3,5 e 7 cordas. 


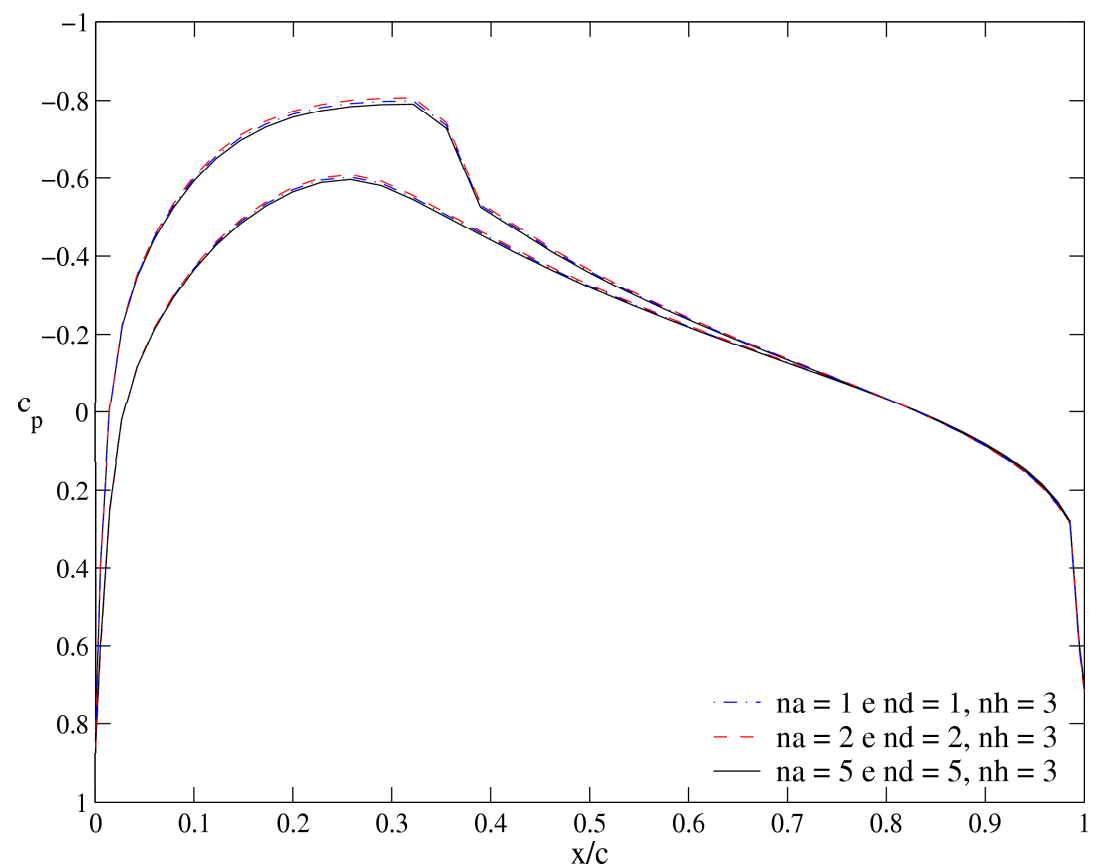

Figura 2.13: Distribuição do coeficiente de pressão para o perfil NACA0012 em $M_{\infty}=0,750$ e $\alpha=1,00^{\circ}$, com a distância antes do bordo de ataque e pós bordo de fuga, $n a$ e $n d$, variando em 1, 2 e 5 cordas. 


\section{Capítulo 3}

\section{Análise de Resultados}

Os resultados apresentados neste capítulo são simulações do programa TSD bidimensional, desenvolvido durante o período de doutoramento e baseado nas descrições da seção 2.5. O objetivo das simulações é testar o método para diferentes tipos de perfis em condições de Mach transônico. A análise é feita principalmente com base na posição e intensidade da onda de choque e na convergência numérica. Testes também foram realizados para casos de números de Mach subsônicos e são apresentados no Apêndice B.

\subsection{Geometria dos Perfis}

Foram utilizados três tipos de perfis para estes testes que são

- RAE2822,

- NACA0012,

- NRL7301,

pois foram os que mais apresentaram dados experimentais nas bibliografias utilizadas durante o projeto. As Figuras 3.1, 3.2 e 3.3 mostram a geometria destes perfis, com dados obtidos do UIUC Airfoil Coordinates Database ${ }^{[36]}$.

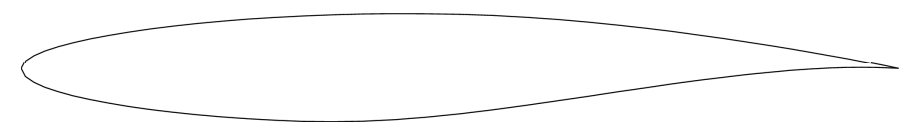

Figura 3.1: Seção do perfil RAE2822. 
Figura 3.2: Seção do perfil NACA0012.

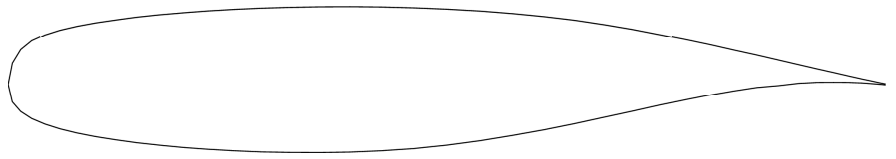

Figura 3.3: Seção do perfil NLR7301.

Os resultados apresentados a seguir foram obtidos para duas condições de simulação numérica: usando a equação de pequenas perturbações transônica com correção de entropia e vorticidade mais a correção de viscosidade (TSD+Ent+Vort+CL); e usando a equação de pequenas perturbações transônica com correção de entropia e vorticidade sem a correção de viscosidade (TSD+Ent+Vort).

O processo de correção para efeitos viscosos foi implementado utilizando a espessura de deslocamento da camada limite. Após 500 iterações do cálculo invíscido a distribuição do número de Mach no perfil foi obtida e utilizada para o cálculo da camada limite. A espessura de deslocamento foi somada às coordenadas do perfil antes da iteração invíscida seguinte. Este procedimento foi repetido a cada 500 iterações até a convergência. Apesar do aumento no tempo de processamento e no número de iterações, houve uma pequena melhora na correlação com os resultados experimentais. Mesmo com esse aumento, ainda preserva-se o baixo custo computacional.

\subsection{Critério de Convergência}

A definição do critério de convergência foi uma das dificuldades encontradas no presente trabalho pois o resíduo da solução potencial apresentava grandes variações que geravam falsas condições de convergência. Por esta razão, durante o processo de obtenção das soluções para os casos que são apresentados neste capítulo foram feitas análises de comportamento das soluções ao longo das iterações.

A Figura 3.4 mostra o comportamento do resíduo ao longo das iterações no caso do perfil RAE2822 com o número de Mach de entrada $\left(M_{\infty}\right)$ igual a 0,729 e o ângulo de ataque $(\alpha)$ 
igual a $2,31^{\circ}$, usando o programa de pequenas perturbações transônica com correção de entropia, vorticidade e viscosidade (TSD+Ent+Vort+CL).

Pode-se observar que a partir da iteração 10.000 ocorre uma oscilação no resíduo. A partir desse instante foi feito um acompanhamento da posição da onda de choque até a iteração 11.610, onde ocorre um salto no valor do resíduo. Quando isso aconteceu a posição do choque avançou a jusante. Isso ocorre novamente na iteração 26.338 seguido de uma convergência suave e uniforme durante 10.000 iterações. A partir da iteração 30.000 as variações na distribuição de pressão se tornaram insignificantes ao longo das iterações como mostra a Figura 3.5. Nessa fase foi feita uma análise na distribuição de pressão a cada 500 iterações a qual não variou significativamente. A solução utilizada para este caso foi a da iteração 36.338

No caso do perfil RAE2822, com o número de Mach do escoamento livre $\left(M_{\infty}\right)$ igual a 0,730 e o ângulo de ataque $(\alpha)$ igual a $3,19^{\circ}$, usando o programa de pequenas perturbações transônica com correção de entropia, vorticidade e viscosidade (TSD +Ent+Vort+CL), foi observado um comportamento diferente. Observa-se que, na Figura 3.6, existem quatro saltos. Cada salto no resíduo representa um deslocamento da onda de choque de um intervalo da malha a jusante. Após 32.000 iterações observa-se que o resíduo se torna oscilatório e não possui comportamento de convergência. A partir desta iteração foi feito um acompanhamento na curva de distribuição de pressão a qual se manteve praticamente constante a partir da iteração 35.000. Este acompanhamento foi feito até a iteração 50.000 até a qual não houve nenhuma variação significativa, como se pode ver na Figura 3.7. Os resultados para este caso foram obtidos da iteração 50.000 .

O critério de parada basicamente foi baseado em dois fenômenos quando o residual da solução potecial estabiliza no seu comportamento e a convergência da distribuição de pressão se estabiliza, nesse momento é feito a parada. Pode-se observar nas figuras 3.4 e 3.5 que o critéio de parada para o caso do perfil RAE2822 em $M_{\infty}=0,729$ e $\alpha=2,31^{\circ}$ foi na iteração 32.000 pois a curva de distribuição de pressão estabiliza e o residual da solução potencial estabiliza em seu comportamento, da mesma maneira no caso do perfil RAE2822 em $M_{\infty}=0,730$ e $\alpha=3,19^{\circ}$ o comportamento do residual da solução potencial se estabiliza a partir da $35.000^{a}$ e a distibuição de pressão também, como pode-se ver nas figuras 3.6 e 3.7 . 


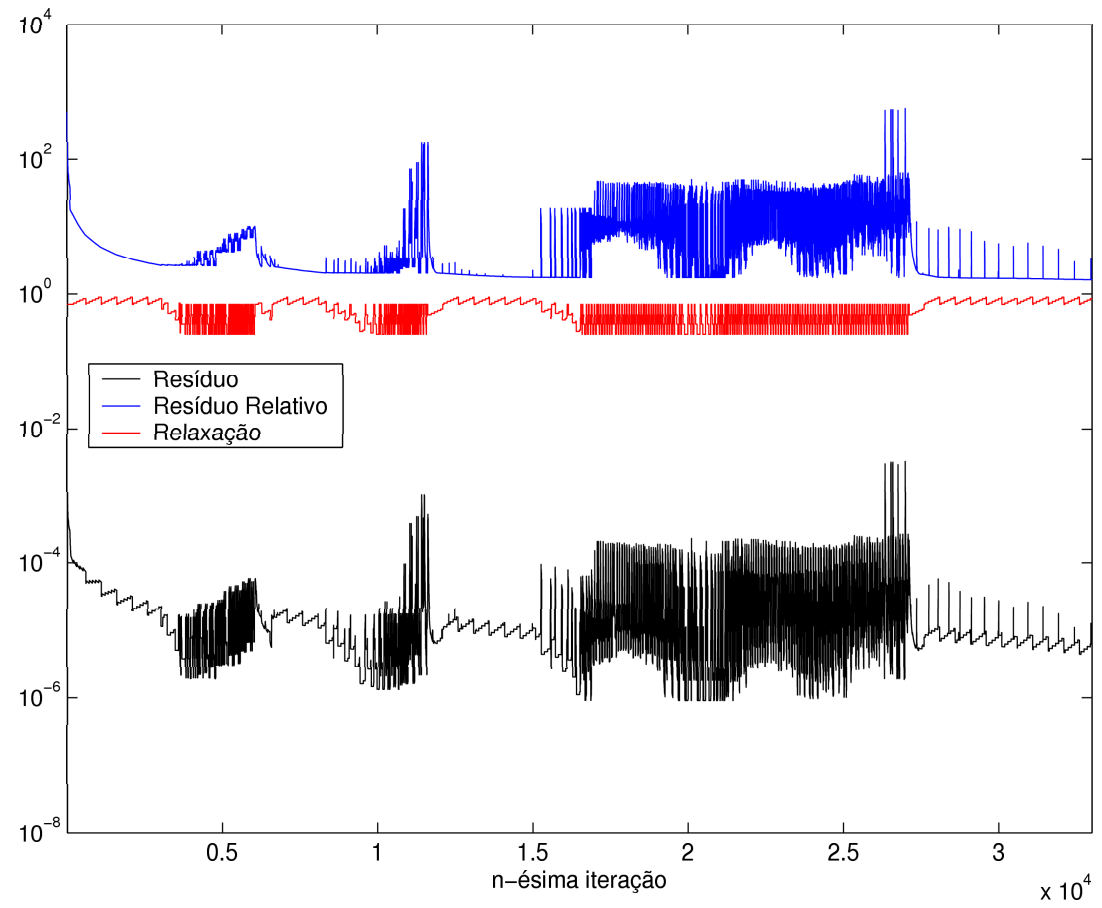

Figura 3.4: Gráfico do resíduo usando TSD + Ent + Vort + CL para seção do perfil RAE2822 $\operatorname{com} M_{\infty}=0,729$ e $\alpha=2,31^{\circ}$.

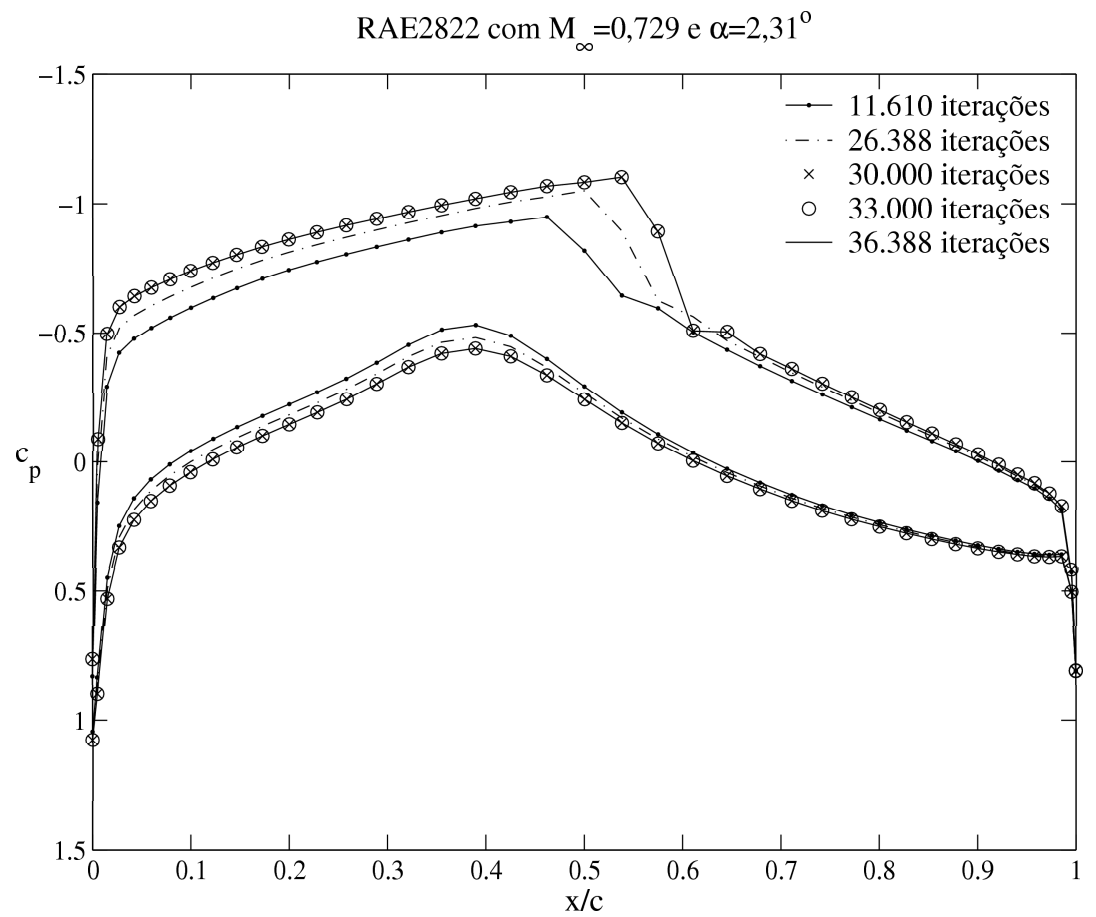

Figura 3.5: Comportamento da distribuição de pressão ao longo das iterações na simulação no perfil RAE2822 com $M_{\infty}=0,729$ e $\alpha=2,31^{\circ}$, usando TSD + Ent + Vort + CL. 


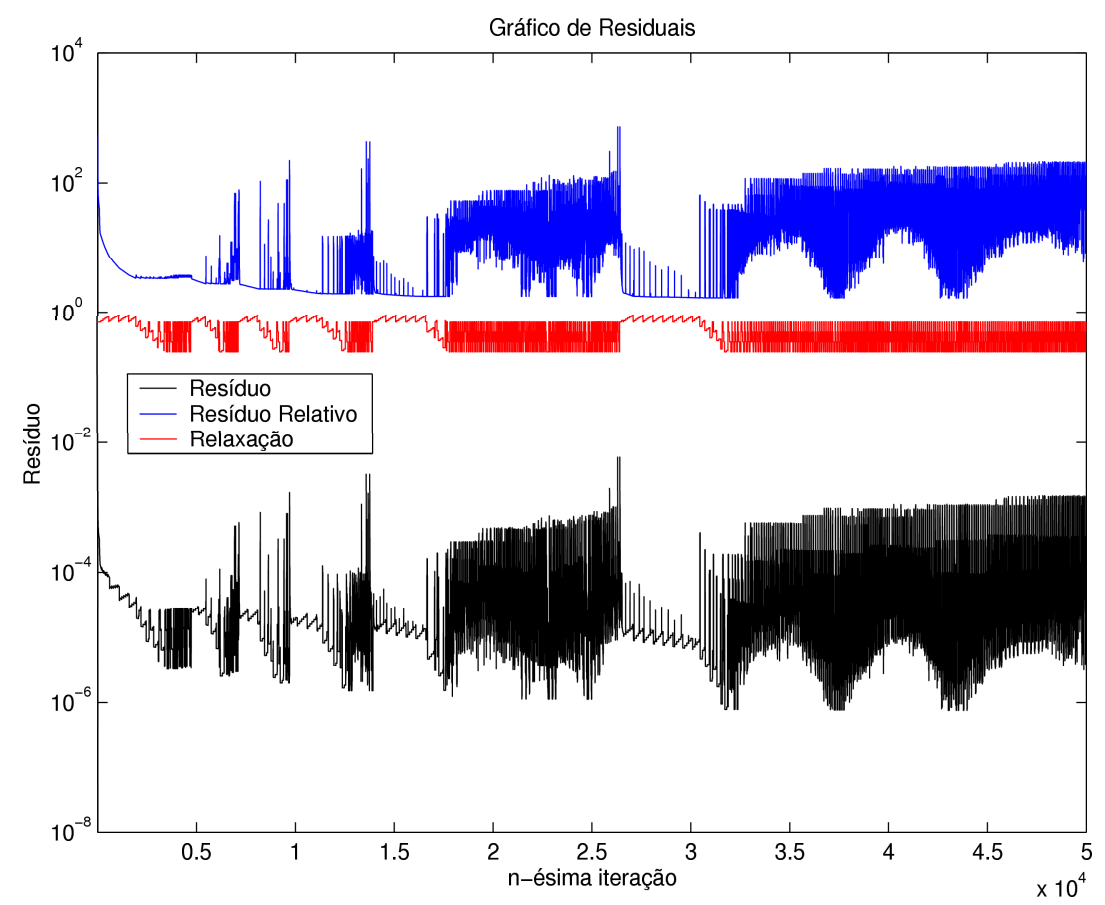

Figura 3.6: Gráfico do resíduo usando TSD+Ent+Vort+CL para seção do perfil RAE2822 com $M_{\infty}=0,730$ e $\alpha=3,19^{\circ}$.

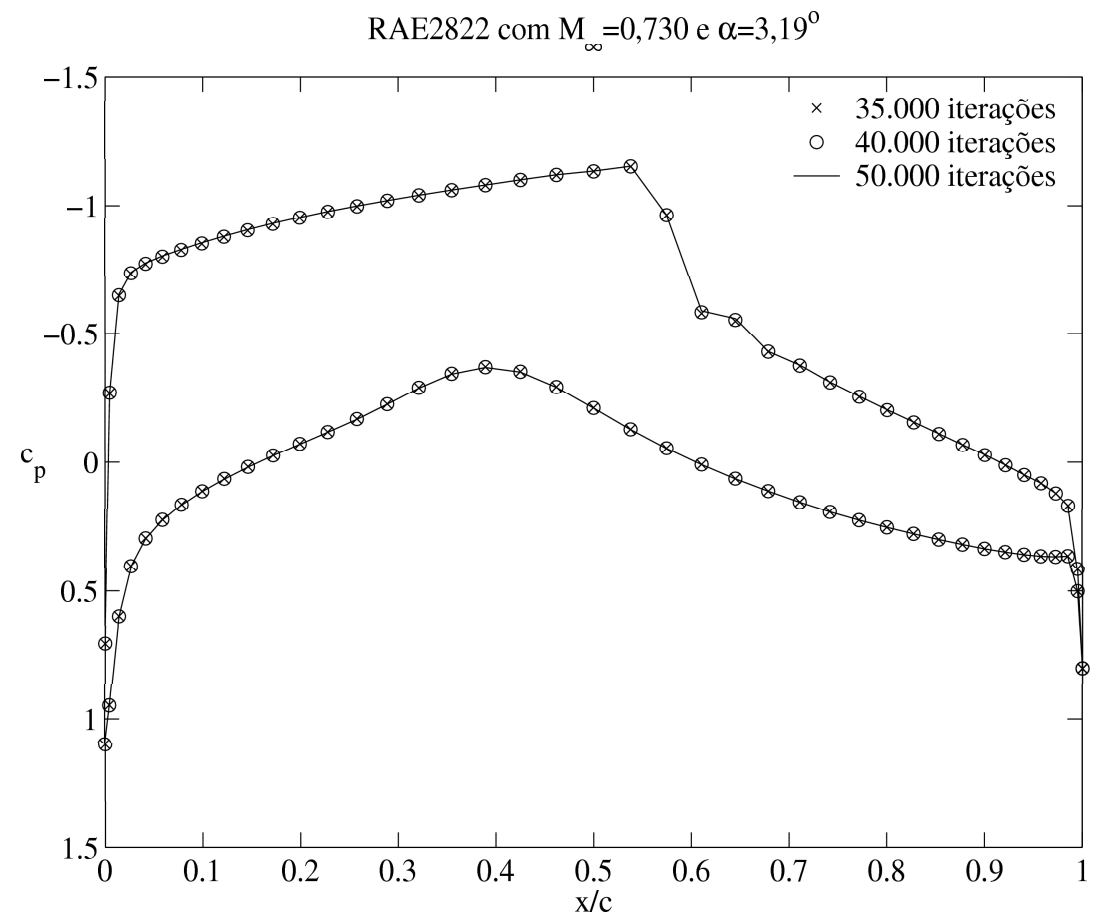

Figura 3.7: Comportamento da distribuição de pressão ao longo das iterações na simulação no perfil RAE2822 com $M_{\infty}=0,730$ e $\alpha=3,19^{\circ}$, usando TSD + Ent + Vort + CL. 
Nos outros casos mostrados neste capítulo foram utilizados os mesmos critérios de parada mostrados nesta sessão. O mesmo critério foi utilizado para o programa de pequenas perturbações transônica com correção de entropia e vorticidade, sem correção de viscosidade (TSD+Ent+Vort).

\subsection{RAE2822}

Primeiramente foi analisado o perfil RAE2822, com o número de Mach de entrada $\left(M_{\infty}\right)$ igual a 0,730 e o ângulo de ataque $(\alpha)$ igual a $3,19^{\circ}$, com o número de Reynolds igual a $8,90 \times 10^{6}$. A Figura 3.8 mostra a distribuição de pressão com correção de viscosidade (TSD+Ent+Vort+CL) e sem correção de viscosidade (TSD+Ent+Vort) sendo comparada a dados experimentais ${ }^{22}$. para este caso.

Pode-se observar que a correção viscosa (TSD+Ent+Vort+CL) desloca a onda de choque a montante e reduz a sua intensidade, porém esta mudança é muito sutil. Variando o número de Mach de entrada $\left(M_{\infty}\right)$ para 0,729 e o ângulo de ataque $(\alpha)$ para $2,31^{\circ}$, com o número de Reynolds igual a 8,90 × $10^{6}$, pode-se notar mais claramente o deslocamento da onda de choque a montante e a redução da sua intensidade, tornando este resultado mais próximo do experimental do que o obtido sem a correção, como pode ser observado na Figura3.9.

Nas Figuras 3.10 e 3.11 nota-se que a espessura de deslocamento, obtida após a convergência da solução usando TSD+Ent+Vort+CL, tem um aumento no gradiente de espessamento logo após o choque no extradorso. Esse espessamento é um dos efeitos responsáveis pelo deslocamento e redução da intensidade do choque e é resultado da abrupta redução no número de Mach através da onda de choque e aumento de pressão.

A Figura 3.11 difere um pouco da Figura 3.10, devido ao número e Mach e ao ângulo de ataque serem um pouco menores, acarretando uma espessura de deslocamento um pouco menor no extradorso e um pouco maior no intradorso. Essas diferenças são mostradas na Figura 3.12.

As Figuras 3.13 e 3.14 mostram o perfil RAE2822 com e sem correção viscosa com o número de Mach de entrada $\left(M_{\infty}\right)$ igual a 0,730 e o ângulo de ataque $(\alpha)$ igual a $3,19^{\circ} \mathrm{e}$ com o número de Mach de entrada $\left(M_{\infty}\right)$ igual a 0,729 e o ângulo de ataque $(\alpha)$ igual a $2,31^{\circ}$, ambos com o número de Reynolds igual a $8,90 \times 10^{6}$. Essas figuras mostram que a 
magnitude da correção parece ser compatível com o desenvolvimento de uma camada limite.

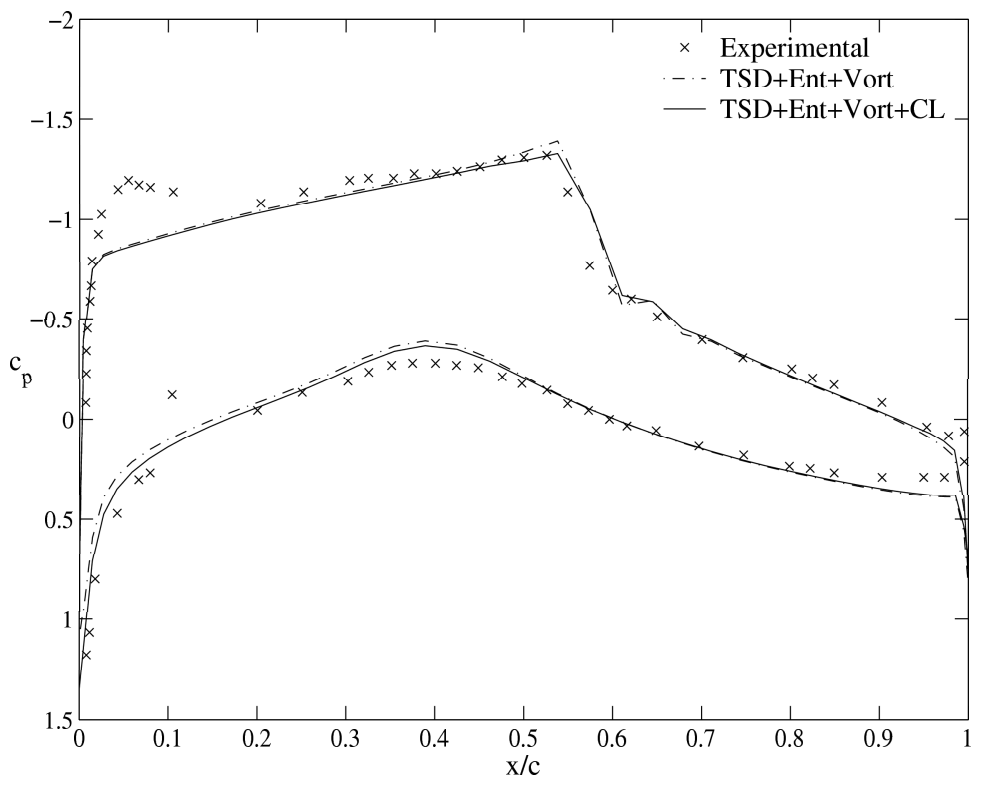

Figura 3.8: Distribuição do coeficiente de pressão para a seção do perfil RAE2822 com $M_{\infty}=0,730$ e $\alpha=3,19^{\circ}$.

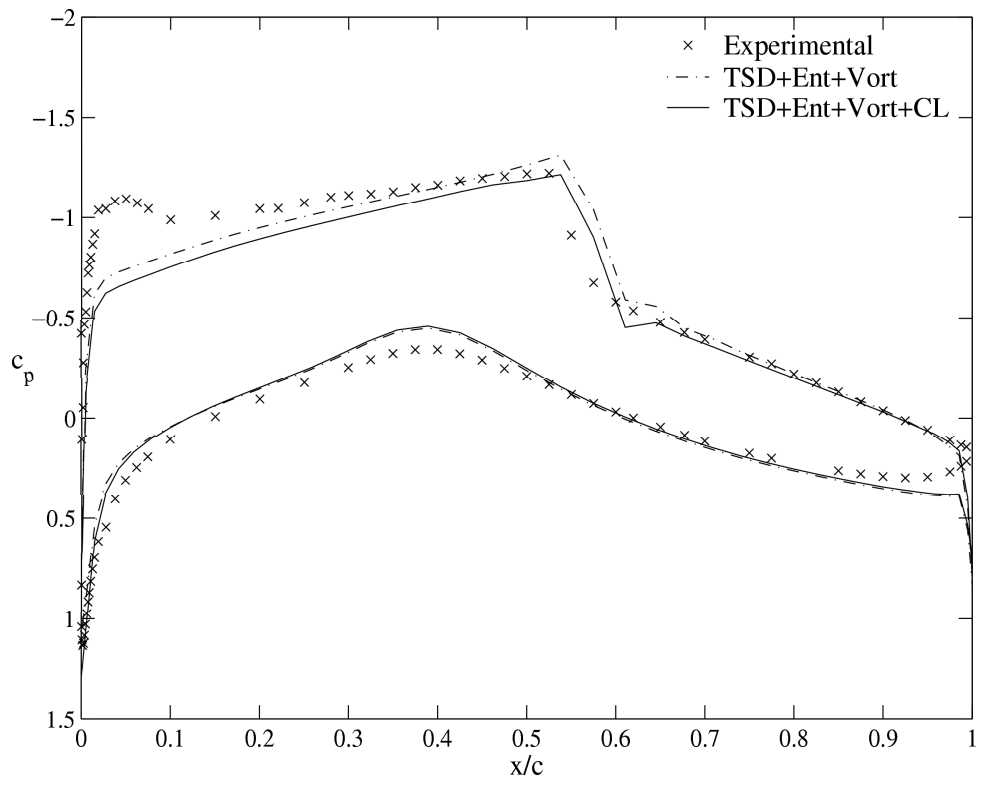

Figura 3.9: Distribuição do coeficiente de pressão para a seção do perfil RAE2822 com $M_{\infty}=0,729$ e $\alpha=2,31^{\circ}$. 


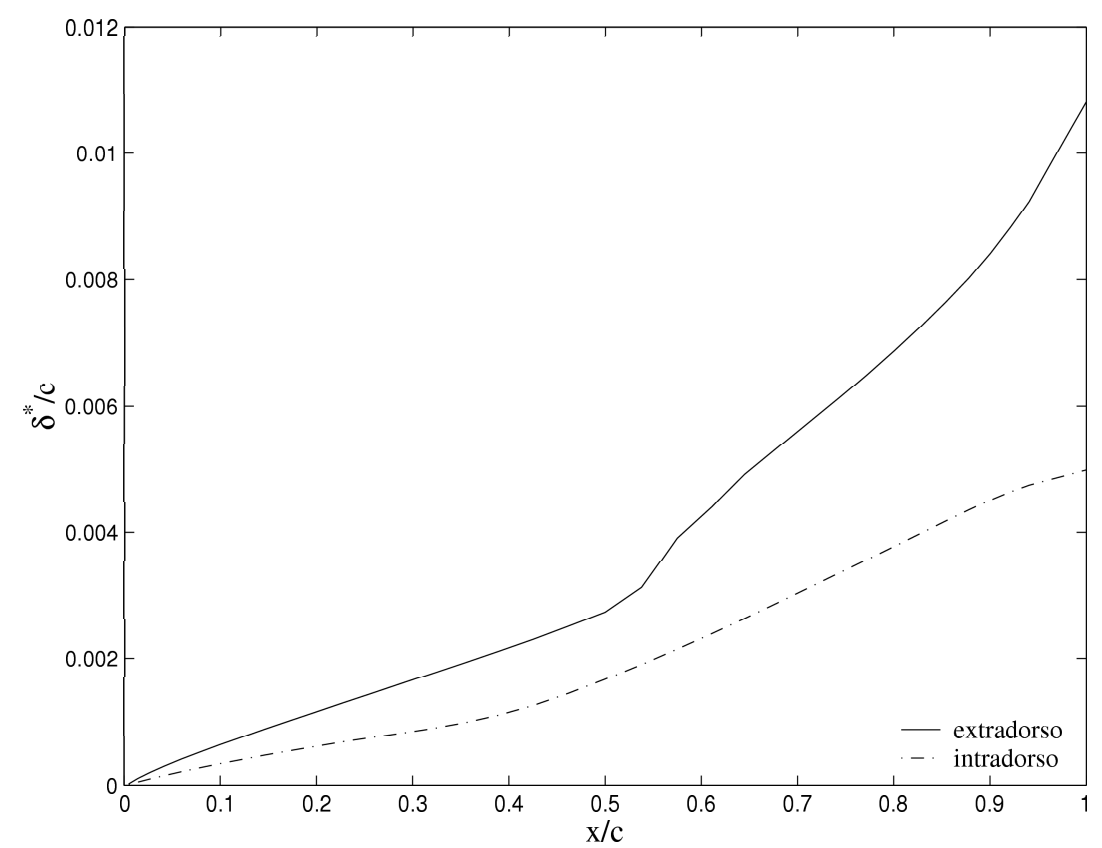

Figura 3.10: Espessura de deslocamento para a seção do perfil RAE2822 com $M_{\infty}=0,730$, $\alpha=3,19^{\circ}$.

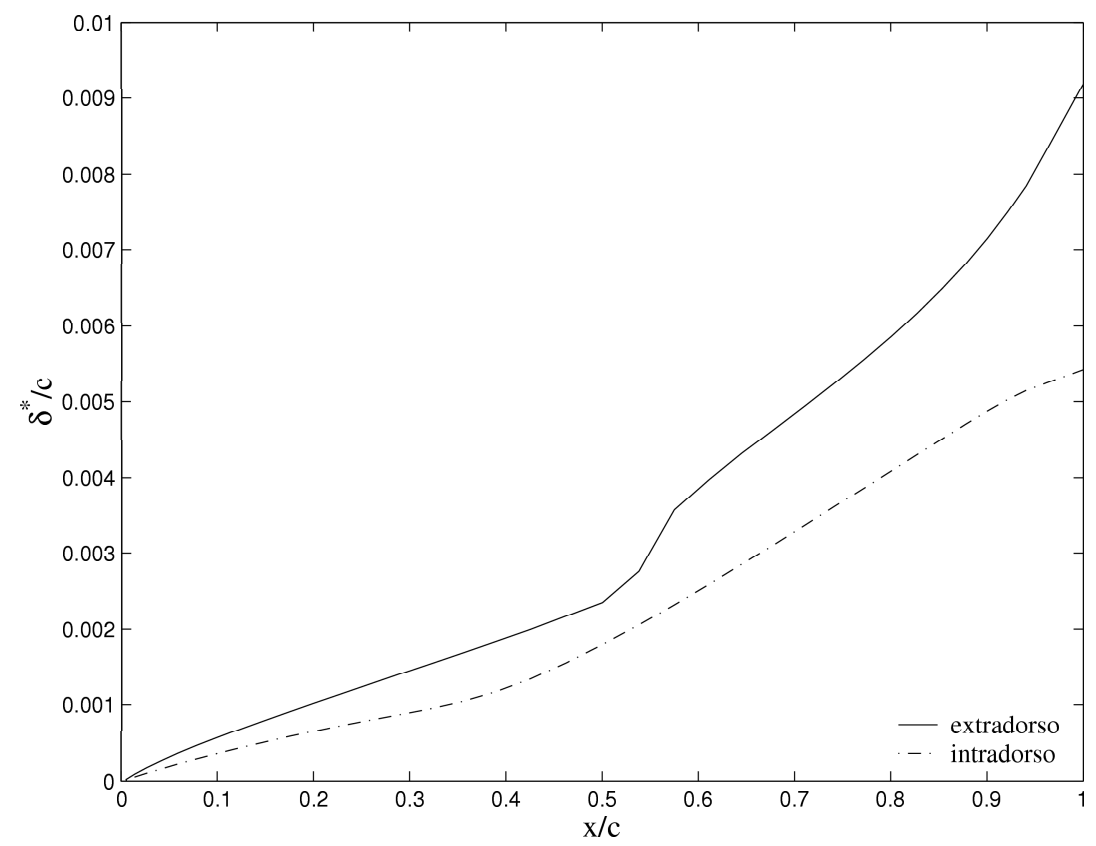

Figura 3.11: Espessura de deslocamento para a seção do perfil RAE2822 $\operatorname{com} M_{\infty}=0,729$, $\alpha=2,31^{\circ}$. 


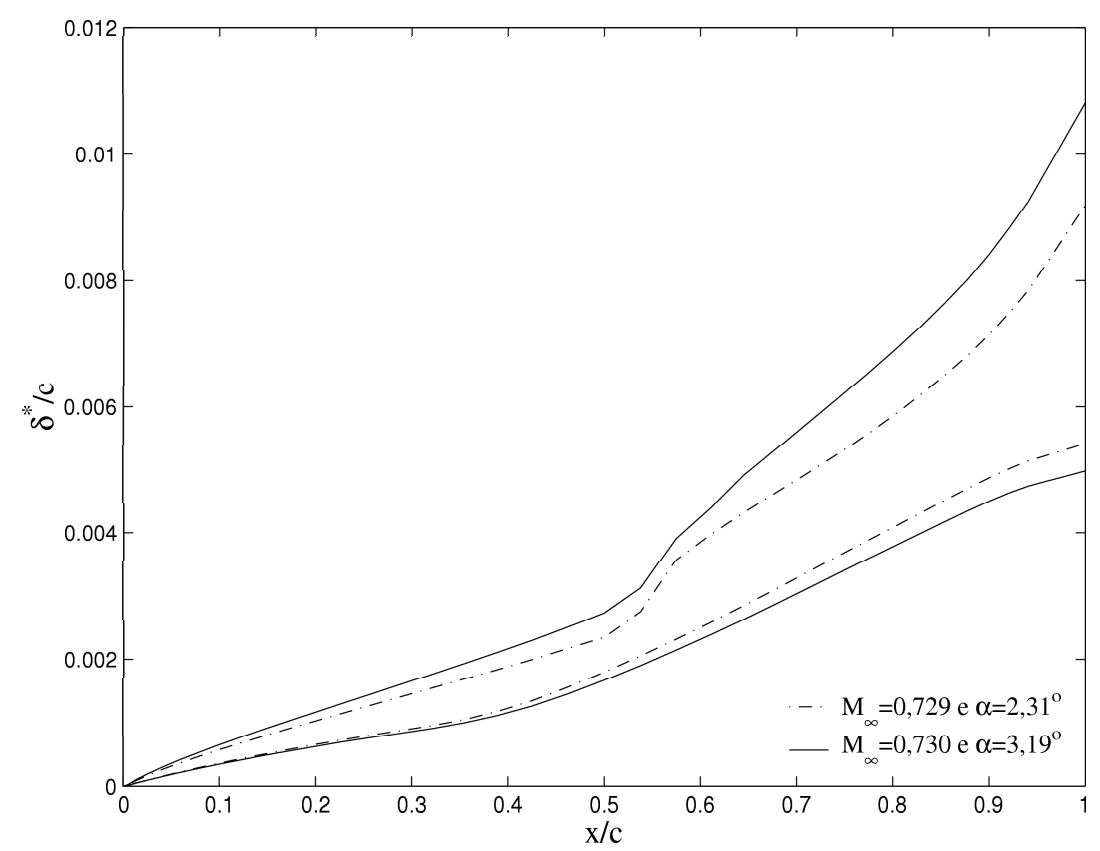

Figura 3.12: Comparação da espessura de deslocamento para a seção do perfil RAE2822 $\operatorname{com} M_{\infty}=0,730, \alpha=3,19^{\circ}$ e $\operatorname{com} M_{\infty}=0,729, \alpha=2,31^{\circ}$.

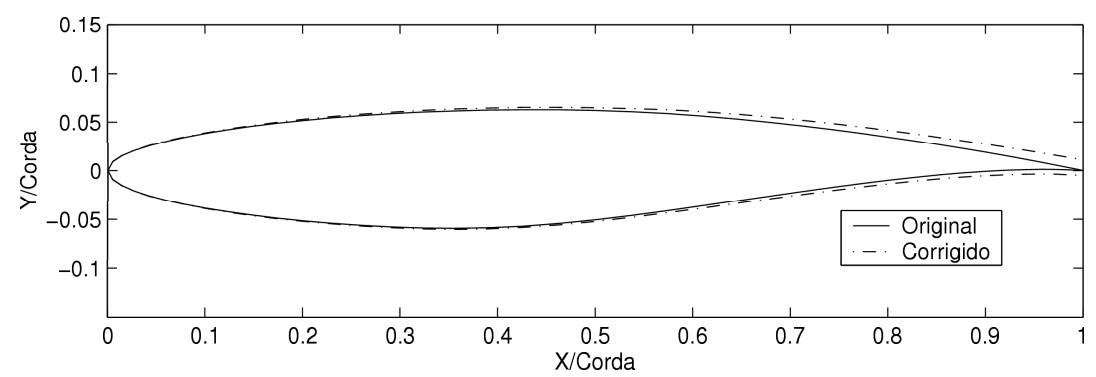

Figura 3.13: Correção da seção do perfil RAE2822 com $M_{\infty}=0,730, \alpha=3,19^{\circ}$, usando espessura de deslocamento.

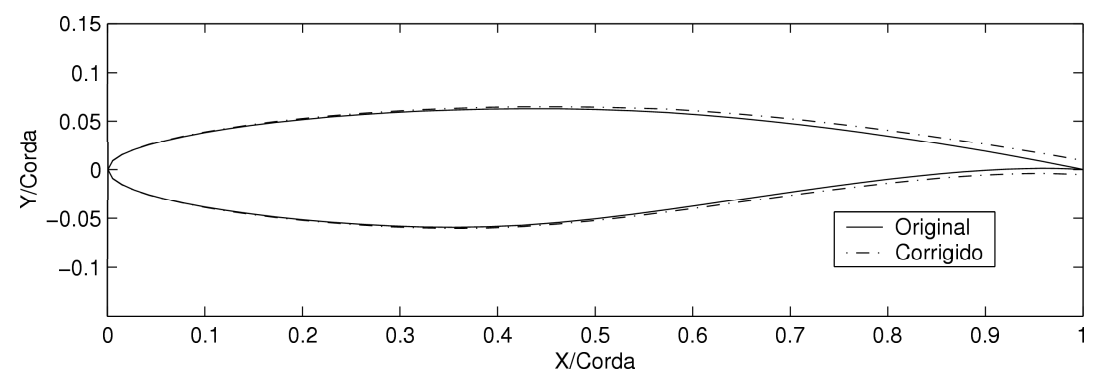

Figura 3.14: Correção da seção do perfil RAE2822 $\operatorname{com} M_{\infty}=0,729, \alpha=2,31^{\circ}$, usando espessura de deslocamento. 
As Figuras 3.15 e 3.16 mostram o campo de velocidade em torno do perfil RAE2822 com o número de Mach de entrada $\left(M_{\infty}\right)$ igual a 0,730 e o ângulo de ataque $(\alpha)$ igual a $3,19^{\circ}$, e com o número de Mach de entrada $\left(M_{\infty}\right)$ igual a 0,729 e o ângulo de ataque $(\alpha)$ igual a $2,31^{\circ}$, ambos com o número de Reynolds igual a $8,90 \times 10^{6}$, respectivamente. A diferença entre as duas figuras é praticamente nula devido as pequenas variações no número de Mach $\left(M_{\infty}\right)$ e no ângulo $(\alpha)$. Isto ocorre também quando se analisa o potencial, como se pode observar nas Figuras 3.17 e 3.18. A diferença só se torna perceptível quando analisamos a distribuição do número de Mach, pois este é mais sensível a pequenas variações como se pode observar nas Figuras 3.19 e 3.20 .

Um fato que também deve ser observado nas Figuras 3.17 e 3.18, devido as condições de contorno descritas na subseção 2.5.6, é a presença dos seguintes fenômenos: a montante do bordo de ataque, na altura do perfil, o escoamento potencial é contínuo. A jusante do bordo de fuga, na altura do perfil, observa-se um salto no potencial. A sustentação gerada pela seção de aerofólio é proporcional a esse salto e aparece devido à imposição da condição de Kutta no bordo de fuga. A condição de Kutta, por sua vez, é imposta pela condição de contorno na esteira do perfil que exige que as velocidades sejam iguais nas linhas superior e inferior da esteira.

Para todas as figuras referentes às análises de campo de velocidade, distribuição de Mach e potencial foram feitas visualizações locais para obter uma melhor análise. A região de domínio computacional é muito maior do que se apresenta nos gráficos.

Nas Figuras 3.19 e 3.20 pode-se observar a distribuição do número de Mach em torno do perfil RAE 2822 com $M_{\infty}=0,730$ e $\alpha=3,19^{\circ}$, e com $M_{\infty}=0,729$ e $\alpha=2,31^{\circ}$, respectivamente. Em ambos os casos pode-se observar claramente a formação da onda de choque somente no extradorso. 


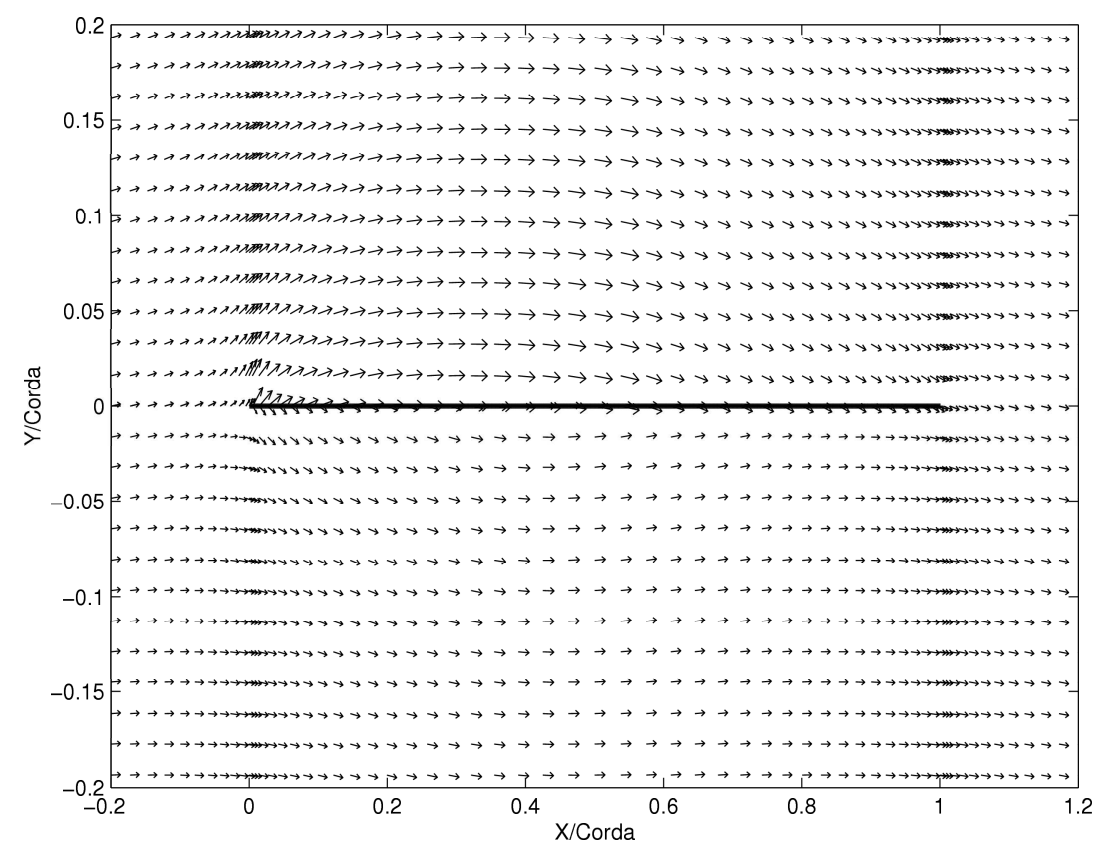

Figura 3.15: Gráfico do campo de velocidade em torno do perfil RAE2822 com $M_{\infty}=0,730$, $\alpha=3,19^{\circ}$, usando TSD+Ent+Vort+CL.

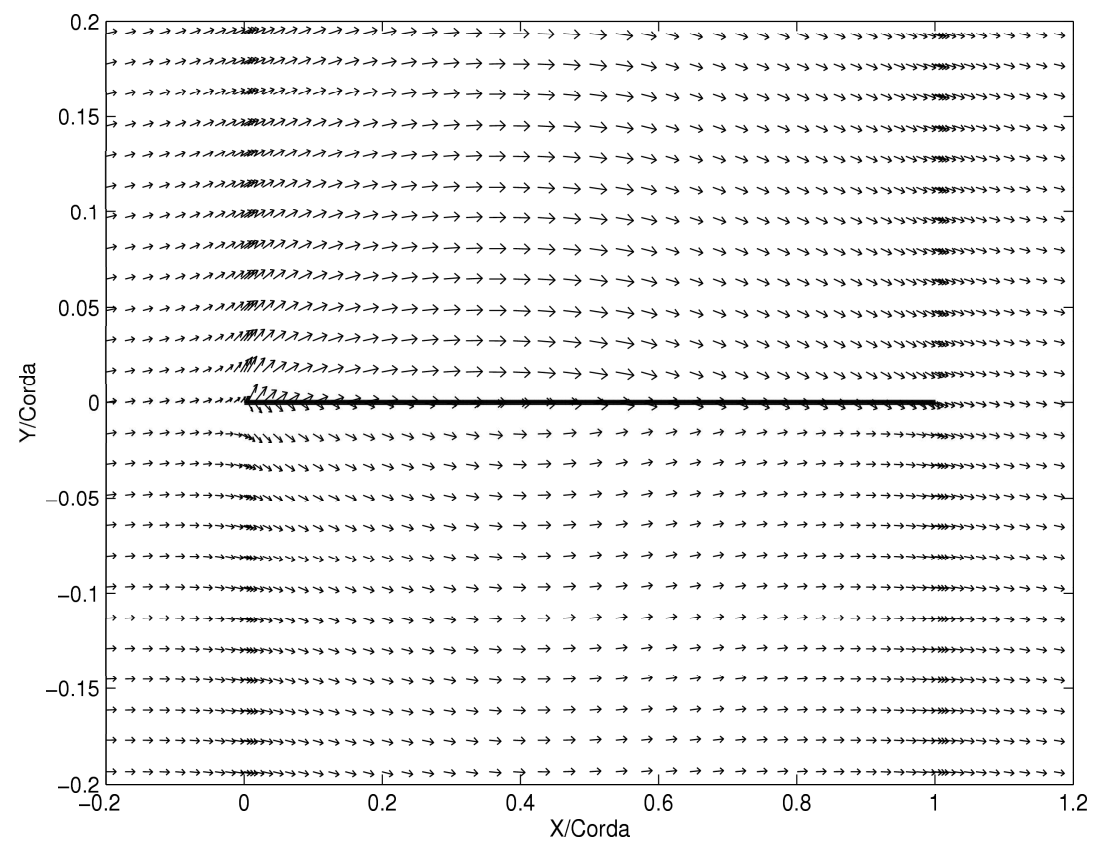

Figura 3.16: Gráfico do campo de velocidade em torno do perfil RAE2822 com $M_{\infty}=0,729$, $\alpha=2,31^{\circ}$, usando TSD+Ent+Vort+CL. 


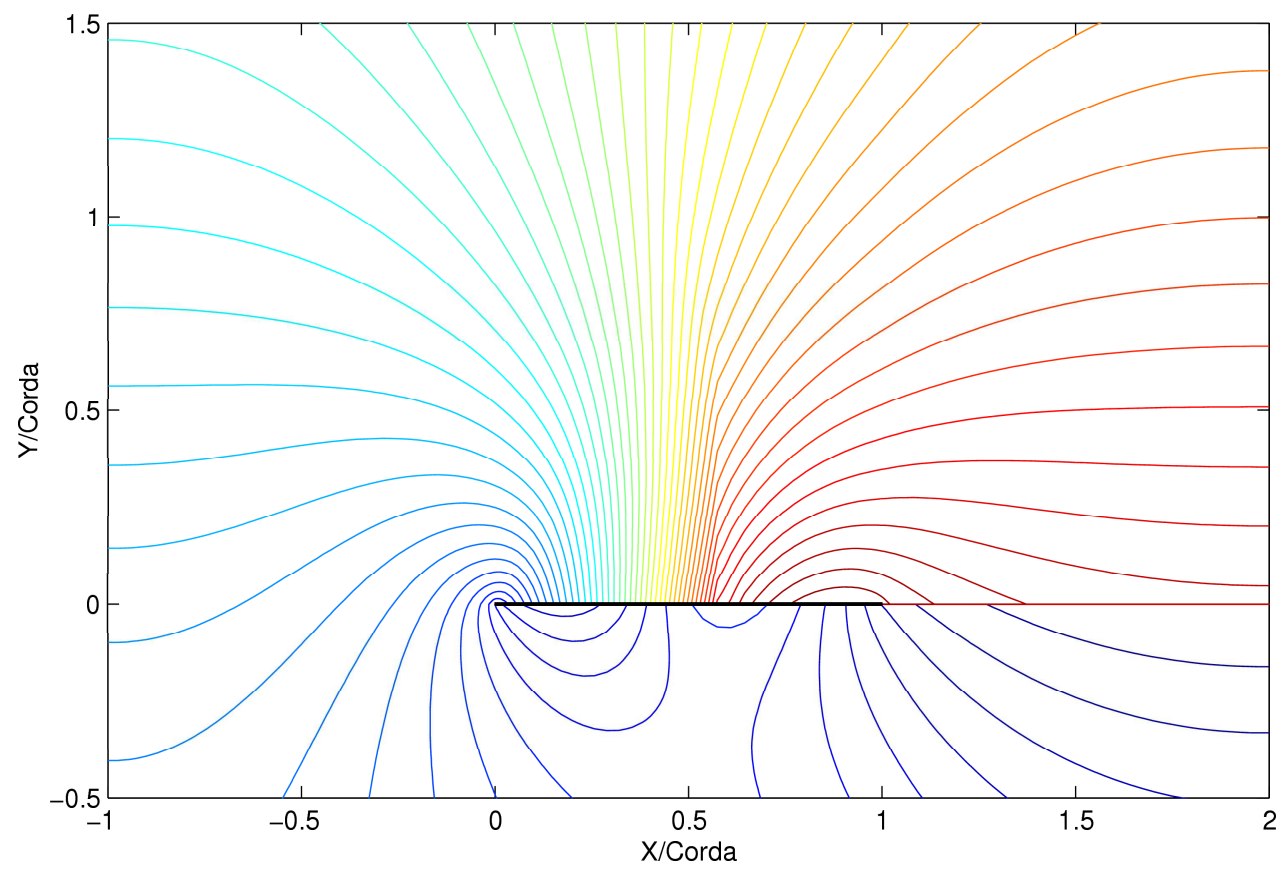

Figura 3.17: Gráfico do potencial em torno do perfil RAE2822 $\operatorname{com} M_{\infty}=0,730, \alpha=3,1^{\circ}$, usando TSD+Ent+Vort+CL.

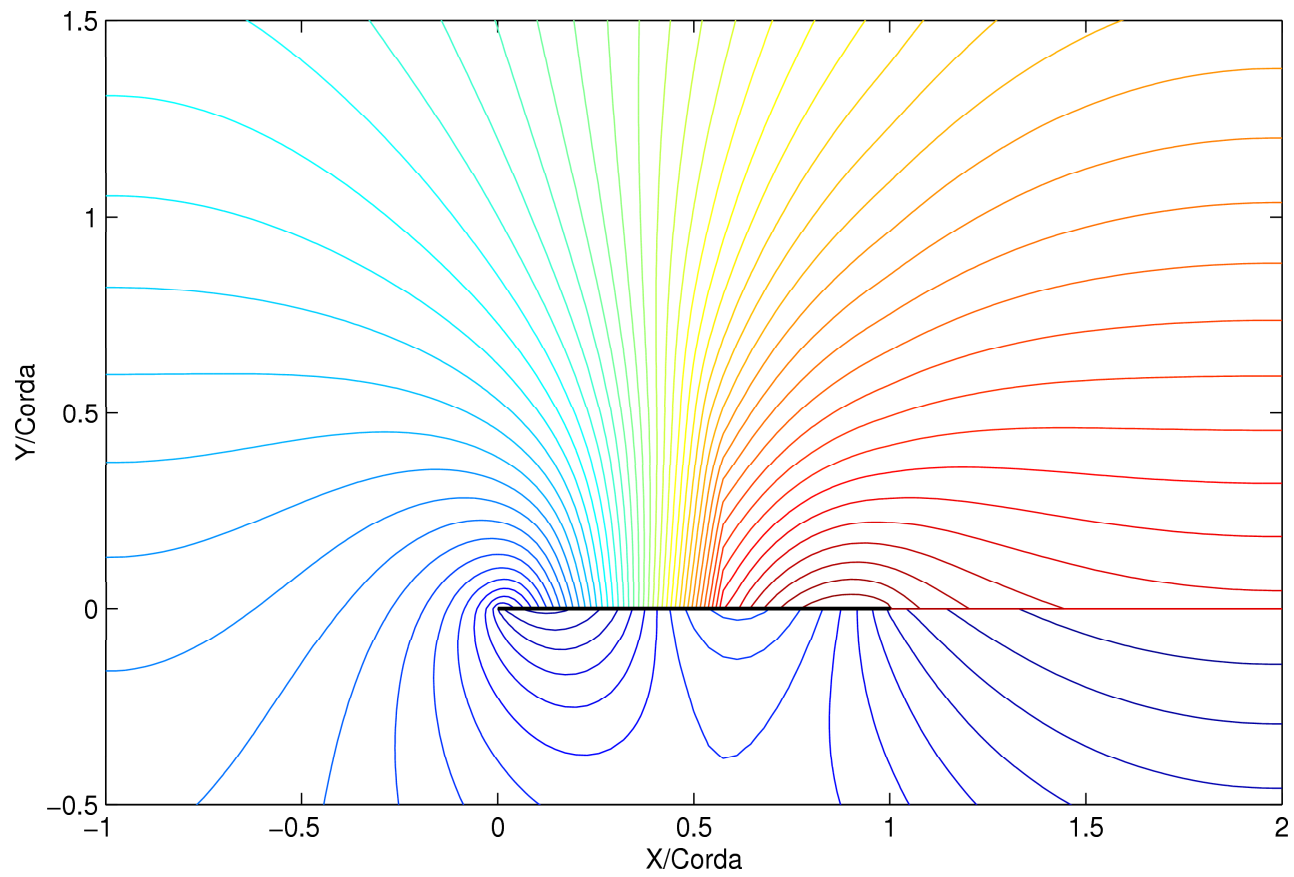

Figura 3.18: Gráfico do potencial em torno do perfil RAE2822 $\operatorname{com} M_{\infty}=0,729, \alpha=2,31^{\circ}$, usando TSD+Ent+Vort+CL. 


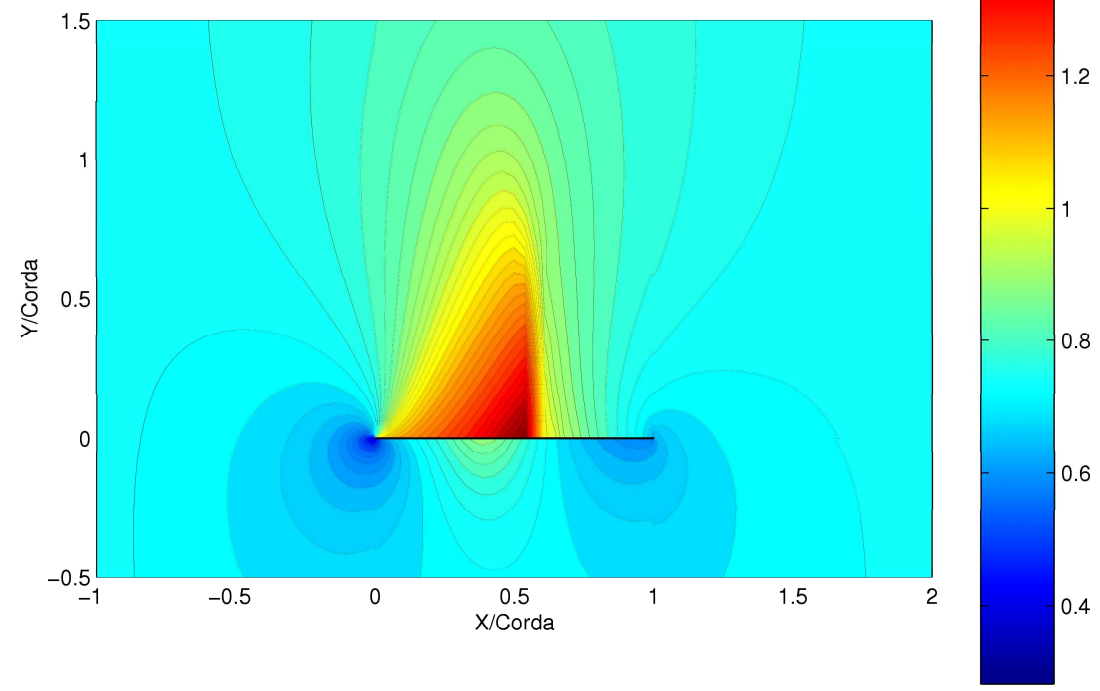

Figura 3.19: Gráfico do número de Mach em torno do perfil RAE2822 $\operatorname{com} M_{\infty}=0,730$, $\alpha=3,19^{\circ}$, usando TSD+Ent+Vort+CL.

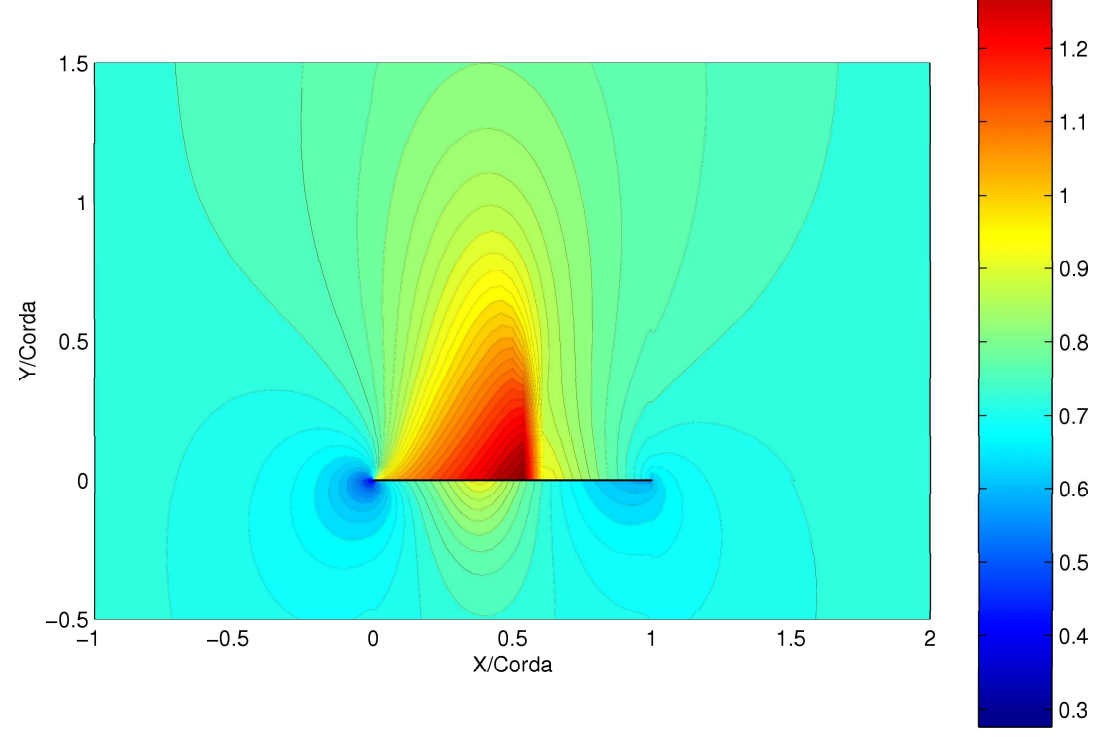

Figura 3.20: Gráfico do número de Mach em torno do perfil RAE2822 $\mathrm{com} M_{\infty}=0,729$, $\alpha=2,31^{\circ}$, usando TSD+Ent+Vort+CL. 


\subsection{NACA0012}

O perfil NACA0012 foi testado com o número de Mach de entrada $\left(M_{\infty}\right)$ igual a 0,750 e os ângulos de ataque $(\alpha)$ iguais a $1,00^{\circ}$ e $3,00^{\circ}$ e com o número de Mach de entrada $\left(M_{\infty}\right)$ igual a 0,800 e o ângulo de ataque $(\alpha)$ igual a $1,25^{\circ}$. Todos os casos obtidos com o perfil NACA0012 nesta seção foram com o número de Reynolds igual a 5,30 x $10^{6}$.

As Figuras 3.21, 3.22 e 3.23 mostram a distribuição de pressão sem correção (TSD+Ent+Vort) e com correção de viscosidade (TSD+Ent+Vort+CL). As duas primeiras distribuições de pressão são comparadas a dados experimentais obtidos da referência [35] e a última é comparada a dados computacionais obtidos pelo programa FLO52 ${ }^{[5]}$. Pode-se observar que os mesmos fenômenos descritos para o perfil RAE2822, ocorrem para o perfil NACA0012, o que era de se esperar dos testes.

Vê-se que quando o perfil NACA0012 com o número de Mach de entrada $\left(M_{\infty}\right)$ igual a 0,800 e o ângulo de ataque $(\alpha)$ igual a $1,25^{\circ}$ usando TSD+Ent+Vort e TSD+Ent+Vort+CL é comparado com os dados computacionais obtidos pelo programa FLO52 ${ }^{[5]}$, as posições da onda de choque, aqui obtidos localizam a montante com relação ao do programa FLO52 ${ }^{[5]}$, além disso o programa FLO52 não captou uma onda de choque fraca no intradorso.

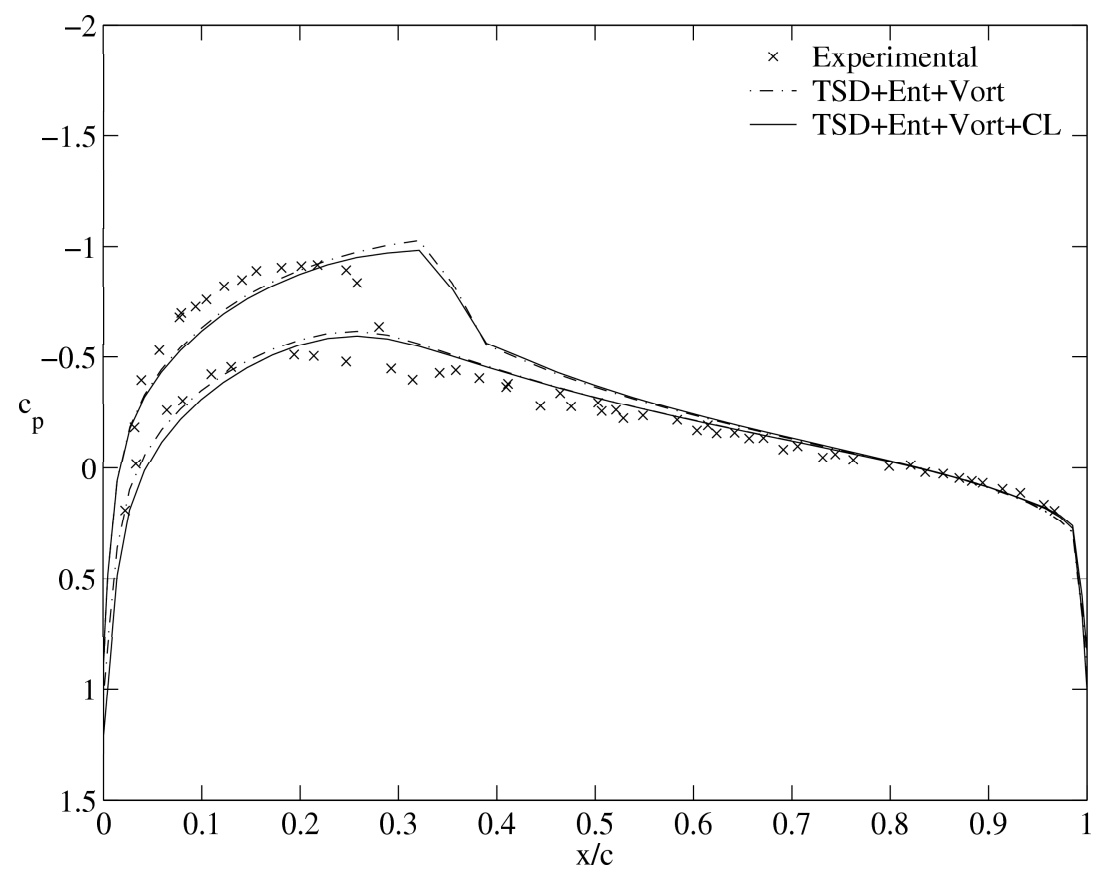

Figura 3.21: Coeficiente de pressão para a seção do perfil NACA0012 com $M_{\infty}=0,750$, $\alpha=1,00^{\circ}$. 
Uma causa possível recai no fato de que nos programas TSD+Ent+Vort e TSD+Ent+Vort+CL foram utilizadas equações na forma não conservativas enquanto que o programa FLO52 foram utilizados equações na forma conservativa. Anderson ${ }^{[3]}$, descreve este fenômeno no seguinte texto:

"Muitas equações de diferenças finitas utilizadas não têm a forma conservativa e, em alguns casos, provaram ter maior acurácia do que as que tem. A importância de se manter a forma conservativa e a alta precisão numa região finita é muito dependente do caso analisado. Todas as formulações consistentes, tendo ou não a forma conservativa, podem fornecer uma representação adequada para a maioria dos problemas se a malha for suficientemente refinada."

Cujo o texto original é:

"Many useful finite-difference equations do not have the conservative property and in a few instances, prove to be more accurate in some sense than those that do. The importance of maintaining the conservation statement with high accuracy over a finite region is highly problem dependent. All consistent formulations, whether or not they have the conservative property, can an adequate representation for most problems if the grid is refined sufficiently."

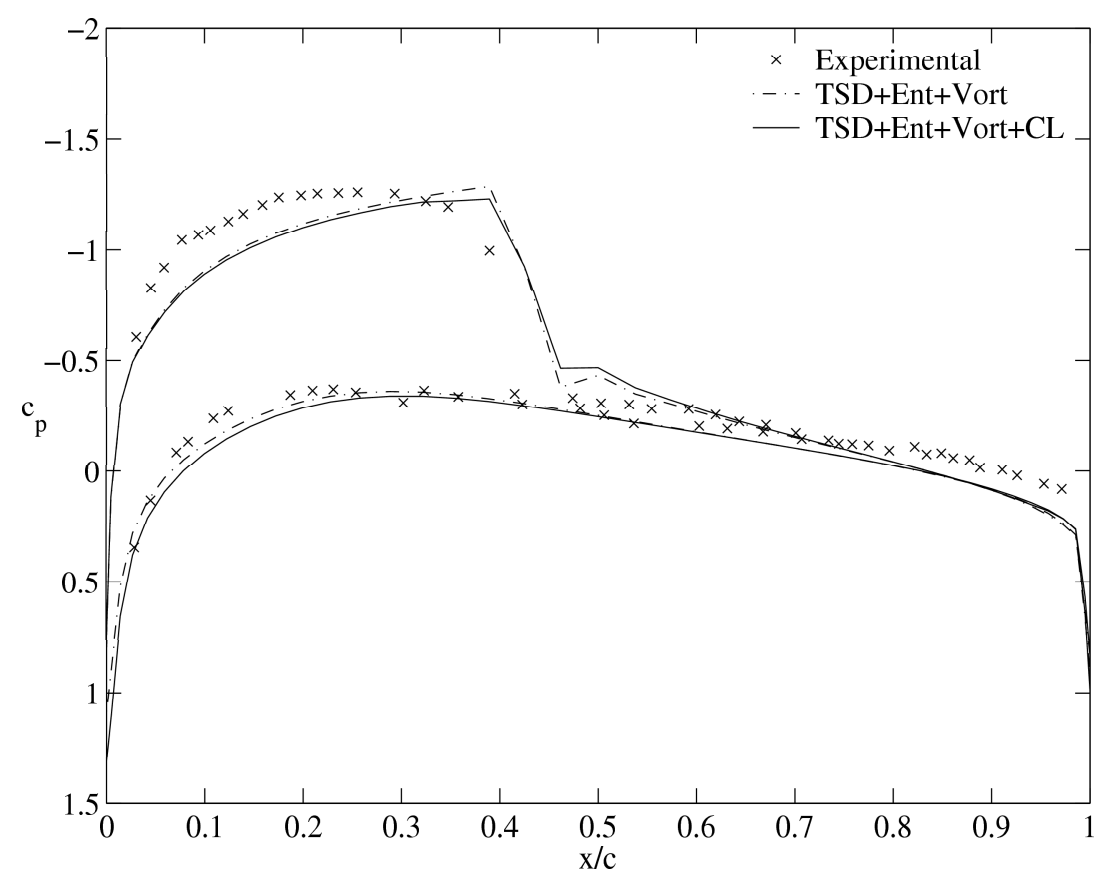

Figura 3.22: Coeficiente de pressão para a seção do perfil NACA0012 com $M_{\infty}=0,750$, $\alpha=3,00^{\circ}$. 


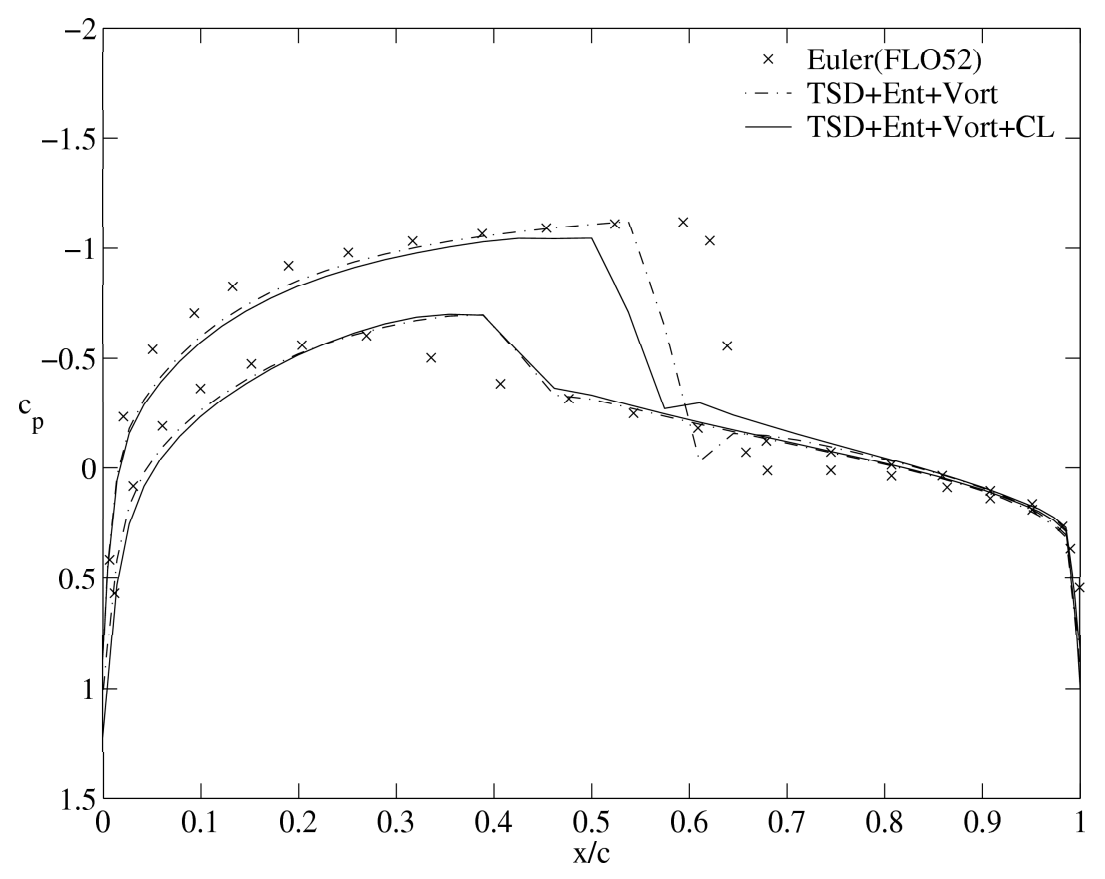

Figura 3.23: Coeficiente de pressão para a seção do perfil NACA0012 com $M_{\infty}=0,800$, $\alpha=1,25^{\circ}$.

As Figuras 3.24, 3.25, 3.26 mostram a espessura de deslocamento obtida após a convergência da solução, usando TSD+Ent+Vort+CL, para o perfil NACA0012 com o número de Mach de entrada $\left(M_{\infty}\right)$ igual a 0,750 e os ângulos de ataque $(\alpha)$ iguais a $1,00^{\circ}$ e $3,00^{\circ} \mathrm{e}$ com o número de Mach de entrada $\left(M_{\infty}\right)$ igual a 0,800 e o ângulo de ataque $(\alpha)$ igual a $1,25^{\circ}$, respectivamente. Mudando o ângulo de ataque já se muda significativamente a espessura de deslocamento e o gradiente de espessamento, como se pode observar na Figura 3.27.

Como no caso do perfil RAE2822, é difícil notar grandes diferenças quando aplicamos a correção viscosa no perfil NACA0012 com o número de Mach de entrada $\left(M_{\infty}\right)$ igual a 0,750 e os ângulos de ataque $(\alpha)$ iguais a $1,00^{\circ}$ e 3,00 e com o número de Mach de entrada $\left(M_{\infty}\right)$ igual a 0,800 e o ângulo de ataque $(\alpha)$ igual a $1,25^{\circ}$, o que pode ser observado nas Figuras $3.28,3.29$ e 3.30 . 


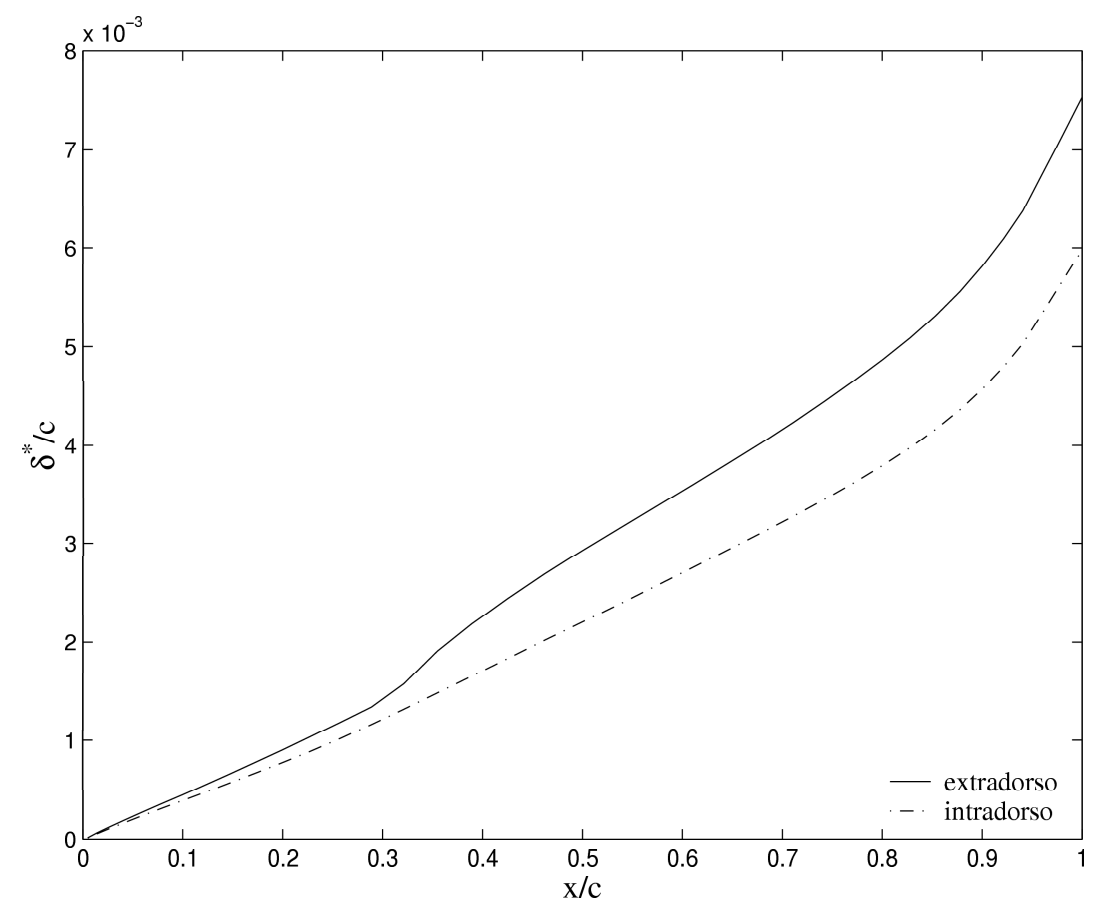

Figura 3.24: Espessura de deslocamento para a seção NACA0012 $\operatorname{com} M_{\infty}=0,750, \alpha=$ $1,00^{\circ}$, usando TSD+Ent+Vort+CL.

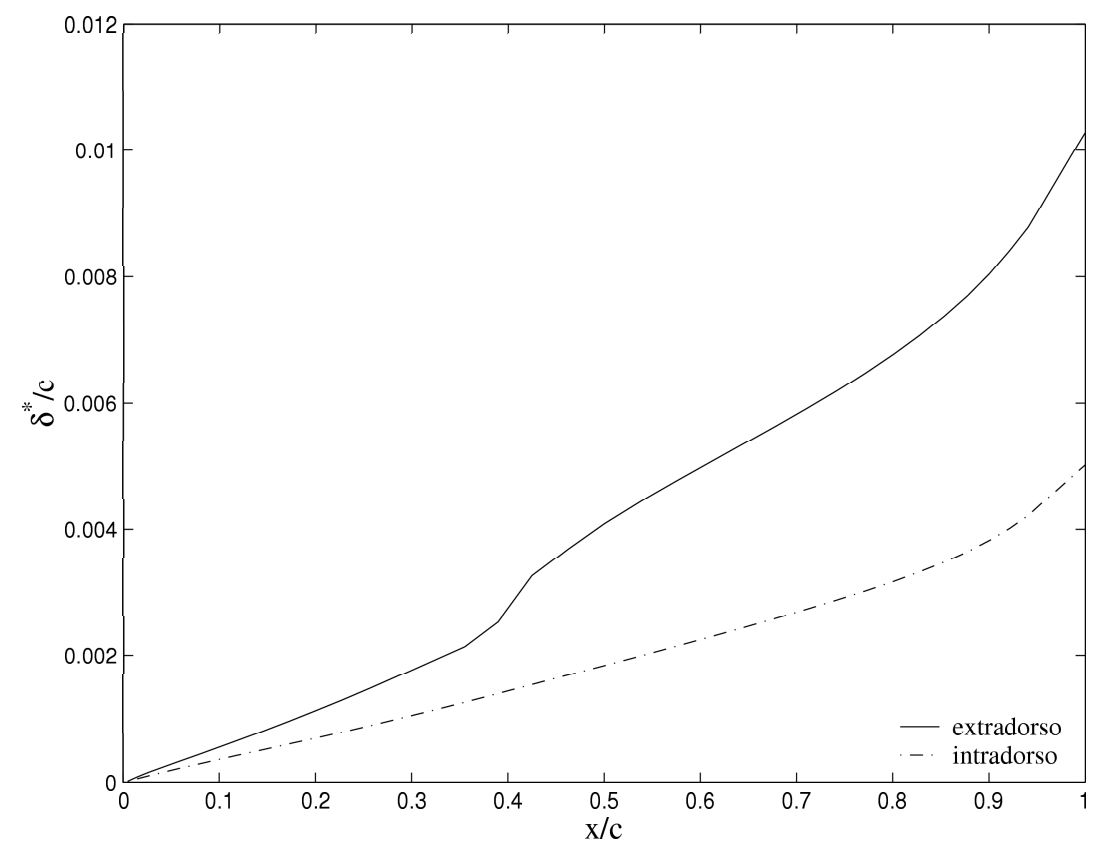

Figura 3.25: Espessura de deslocamento para a seção NACA0012 $\operatorname{com} M_{\infty}=0,750, \alpha=$ $1,00^{\circ}$, usando TSD+Ent+Vort+CL. 


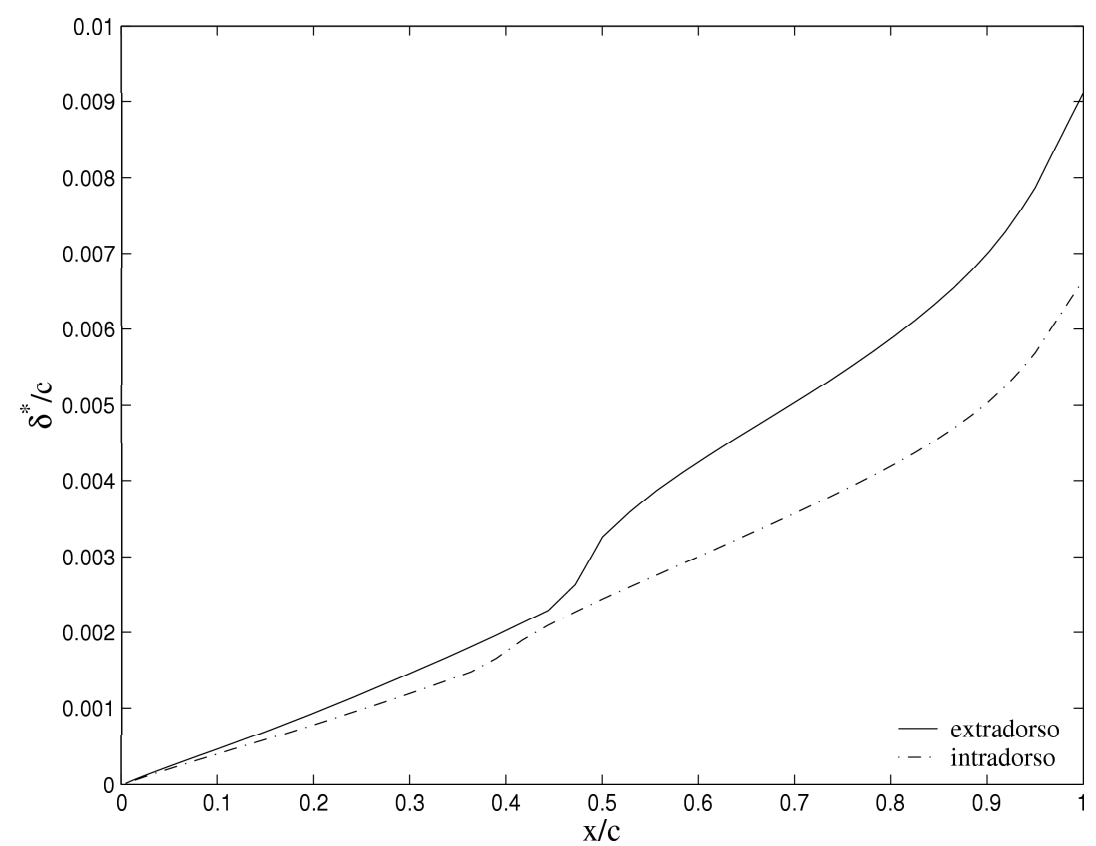

Figura 3.26: Espessura de deslocamento para a seção NACA0012 $\operatorname{com} M_{\infty}=0,800, \alpha=$ $1,25^{\circ}$, usando TSD+Ent+Vort+CL.

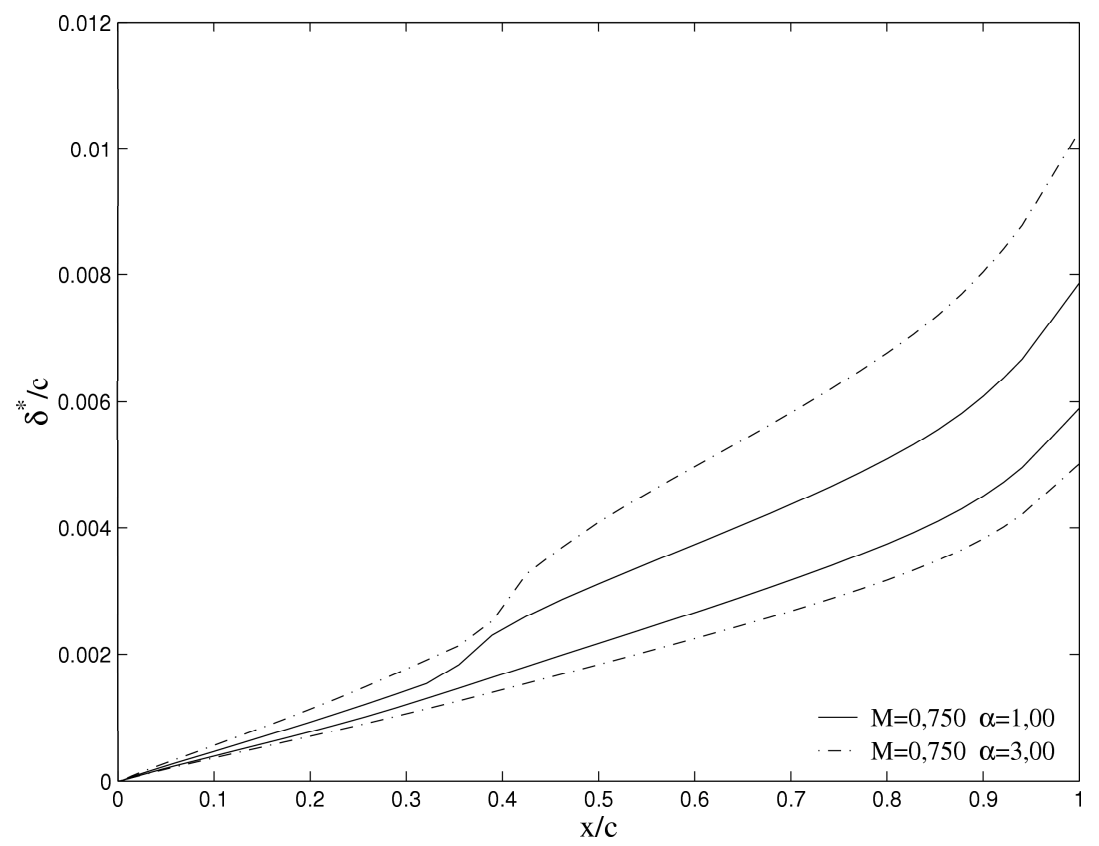

Figura 3.27: Comparação da espessura de deslocamento para a seção do perfil NACA0012 com $M_{\infty}=0,750, \operatorname{com} \alpha=1,00^{\circ}$ e $\alpha=3,00^{\circ}$. 


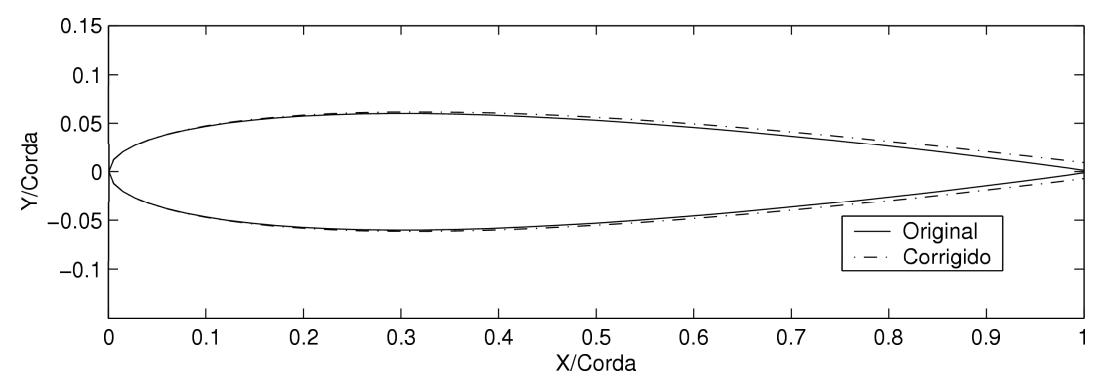

Figura 3.28: Correção da seção do perfil NACA0012 $\operatorname{com} M_{\infty}=0,750, \alpha=1,00^{\circ}$, usando espessura de deslocamento.

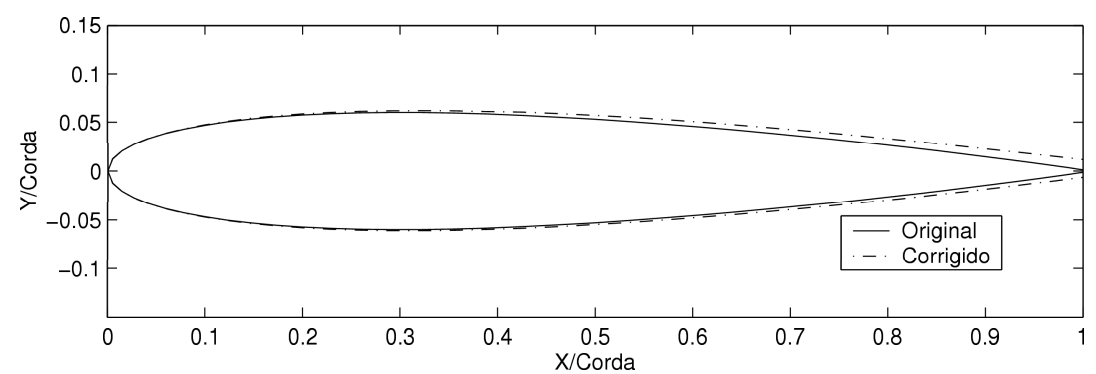

Figura 3.29: Correção da seção do perfil NACA0012 $\operatorname{com} M_{\infty}=0,750, \alpha=3,00^{\circ}$, usando espessura de deslocamento.

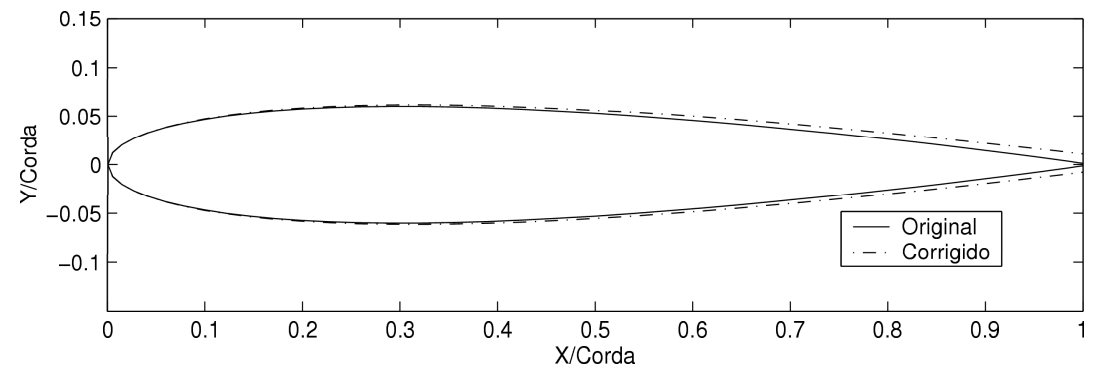

Figura 3.30: Correção da seção do perfil NACA0012 $\operatorname{com} M_{\infty}=0,800, \alpha=1,25^{\circ}$, usando espessura de deslocamento.

Devido ao fato de que as variações são pequenas, os resultados que se tornam interessantes de serem analisados, além da distribuição de pressão, é a distribuição do número de Mach. Novamente se observa que com uma pequena mudança de ângulo de ataque a intensidade e a posição da onda de choque mudam significativamente como mostram as Figuras 3.31 e 3.32. Pode-se observar que com uma variação de 0,05 Mach a intensidade do choque aumenta significativamente, como mostra a Figura 3.33. 


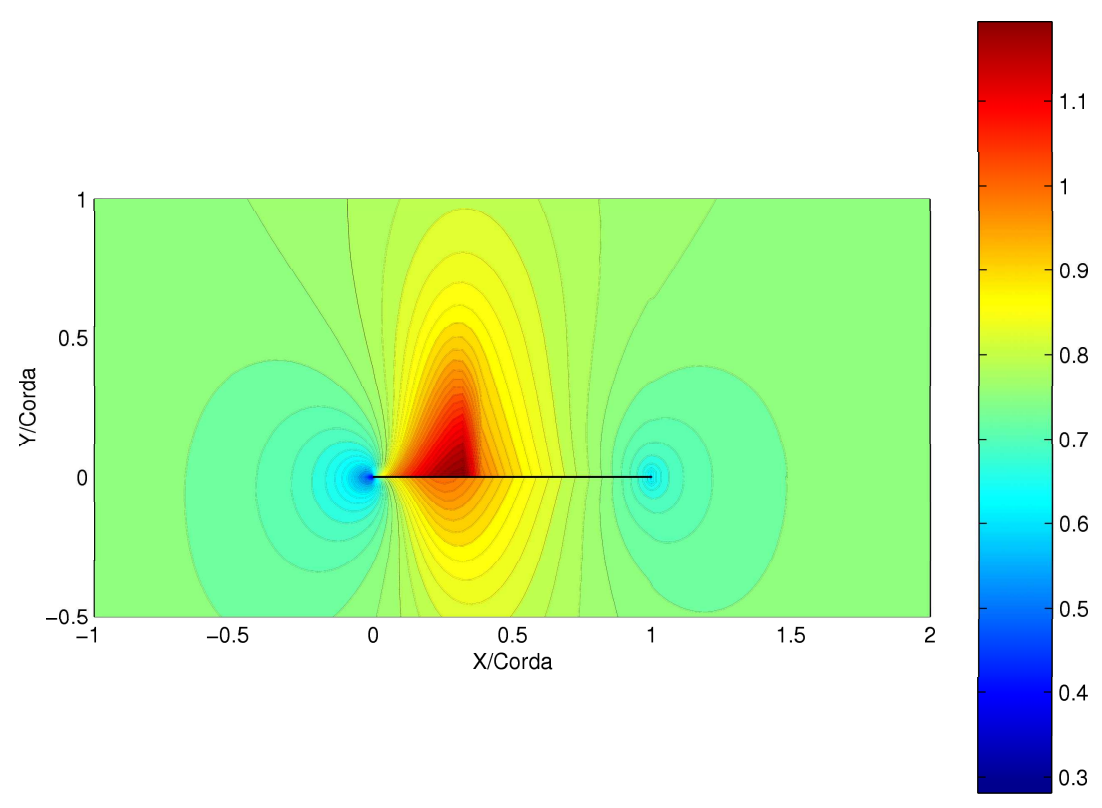

Figura 3.31: Gráfico do número de Mach em torno do perfil NACA0012 com $M_{\infty}=0,750$, $\alpha=1,00^{\circ}$, usando TSD+Ent+Vort+CL.

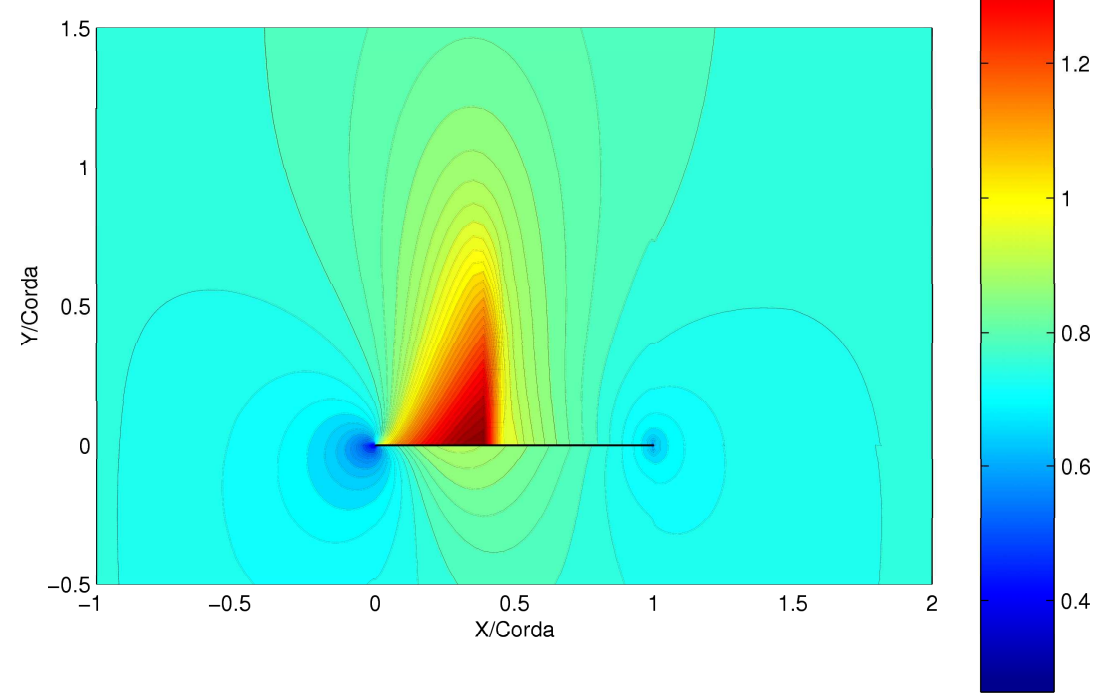

Figura 3.32: Gráfico do número de Mach em torno do perfil NACA0012 com $M_{\infty}=0,750$, $\alpha=3,00^{\circ}$, usando TSD+Ent+Vort+CL. 


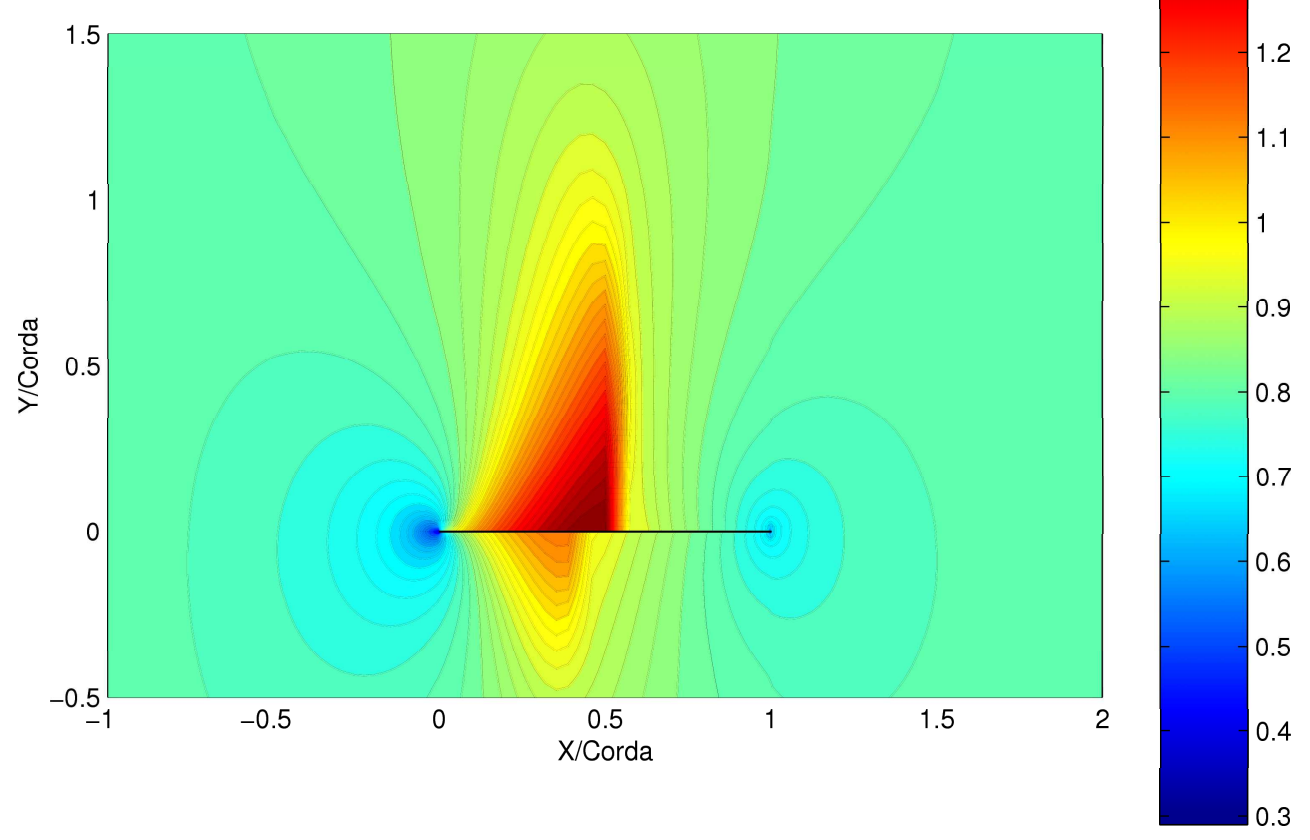

Figura 3.33: Gráfico do número de Mach em torno do perfil NACA0012 com $M_{\infty}=0,800$, $\alpha=1,25^{\circ}$, usando TSD+Ent+Vort+CL.

\subsection{NLR7301}

O terceiro perfil analisado é o NLR7301. As Figuras 3.34 e 3.35 mostram a distribuição de pressão sem correção (TSD+Ent+Vort) e com correção de viscosidade (TSD+Ent+Vort$+\mathrm{CL})$, com o número de Mach de entrada $\left(M_{\infty}\right)$ igual a 0,752 e o ângulo de ataque $(\alpha)$ igual a $0,37^{\circ}$ e Mach de entrada $\left(M_{\infty}\right)$ igual a 0,753 e o ângulo de ataque $(\alpha)$ igual a $-0,08^{\circ}$, respectivamente, e todos com o número de Reynolds igual a $8,20 \times 10^{6}$. Os resultados são comparadas a dados experimentais obtidos da referência [22]. Pode-se também observar a formação da onda de choque no extradorso e uma onda de choque fraca no intradorso em ambos os casos. 


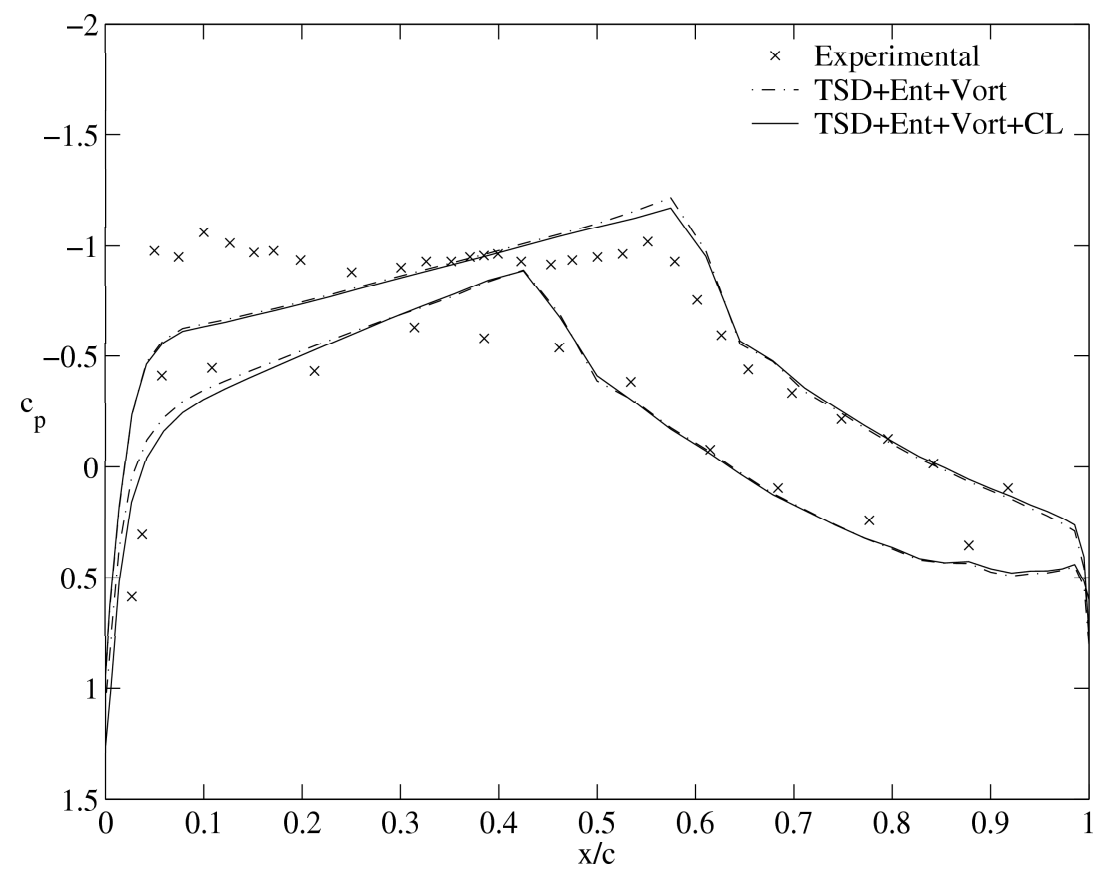

Figura 3.34: Coeficiente de pressão para a seção do perfil NLR7301 com $M_{\infty}=0,752$, $\alpha=0,37^{\circ}$.

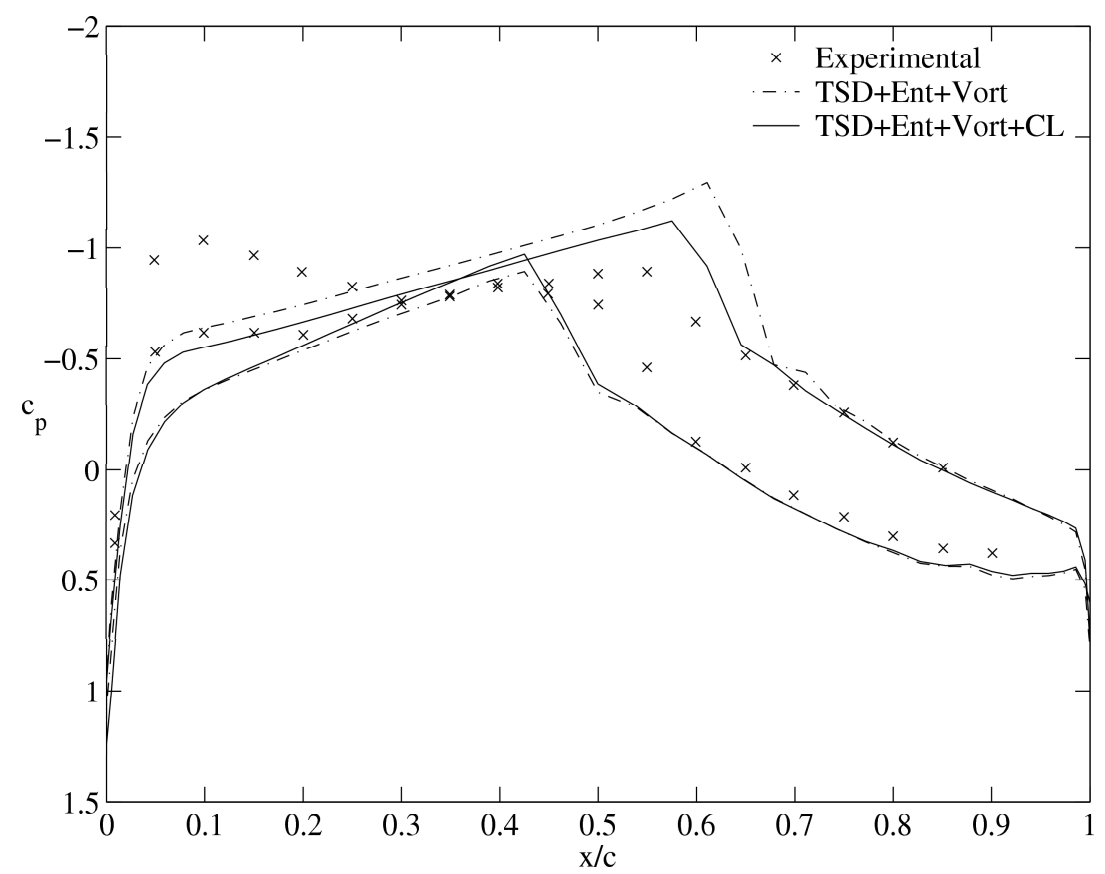

Figura 3.35: Coeficiente de pressão para a seção do perfil NLR7301 com $M_{\infty}=0,753$, $\alpha=-0,08^{\circ}$. 
Nem sempre a espessura de deslocamento é estritamente crescente, nos casos analisados para o perfil NLR7301 ocorre um comportamento diferente, pois ela começa crescente mas perto do bordo de fuga ela se torna decrescente como se pode observar nas Figuras 3.36 e 3.37 .

Analisando a distribuição de Mach em torno do perfil NLR7301 para os casos descritos acima, pode-se observar mais claramente a formação da onda de choque no extradorso e uma onda de choque fraca no intradorso. Além disso, uma melhor visualização da intensidade das ondas de choque pode ser observada. 


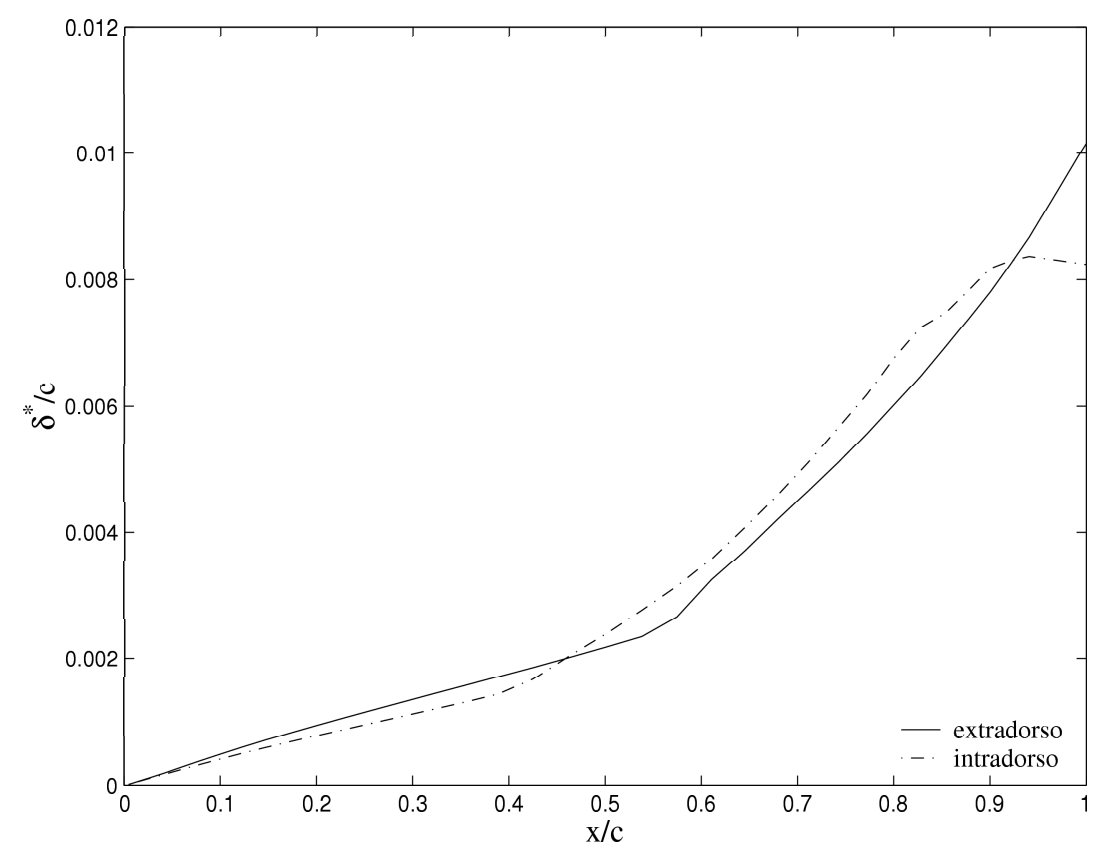

Figura 3.36: Espessura de deslocamento para a seção do perfil NLR7301 $\operatorname{com} M_{\infty}=0,752$, $\alpha=0,37^{\circ}$. usando TSD+Ent+Vort+CL.

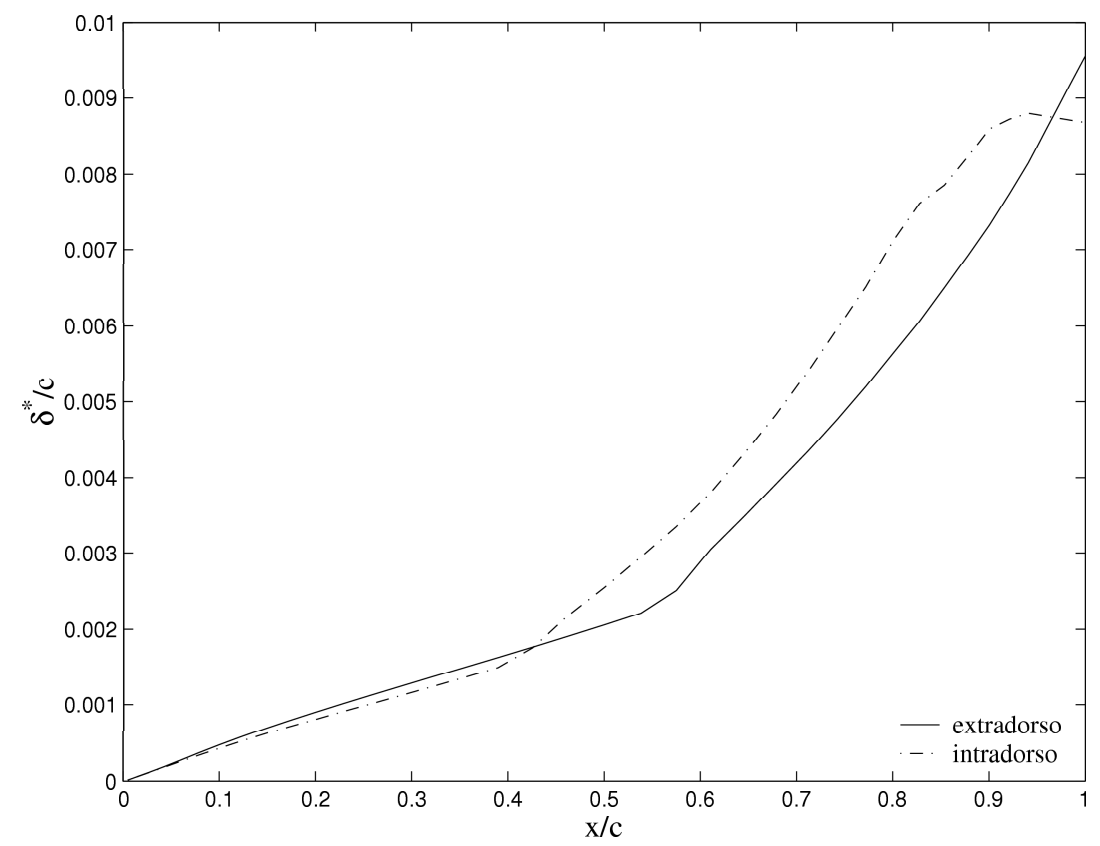

Figura 3.37: Espessura de deslocamento para a seção NLR7301 com $M_{\infty}=0,753, \alpha=$ $-0,08^{\circ}$, usando TSD+Ent+Vort+CL. 


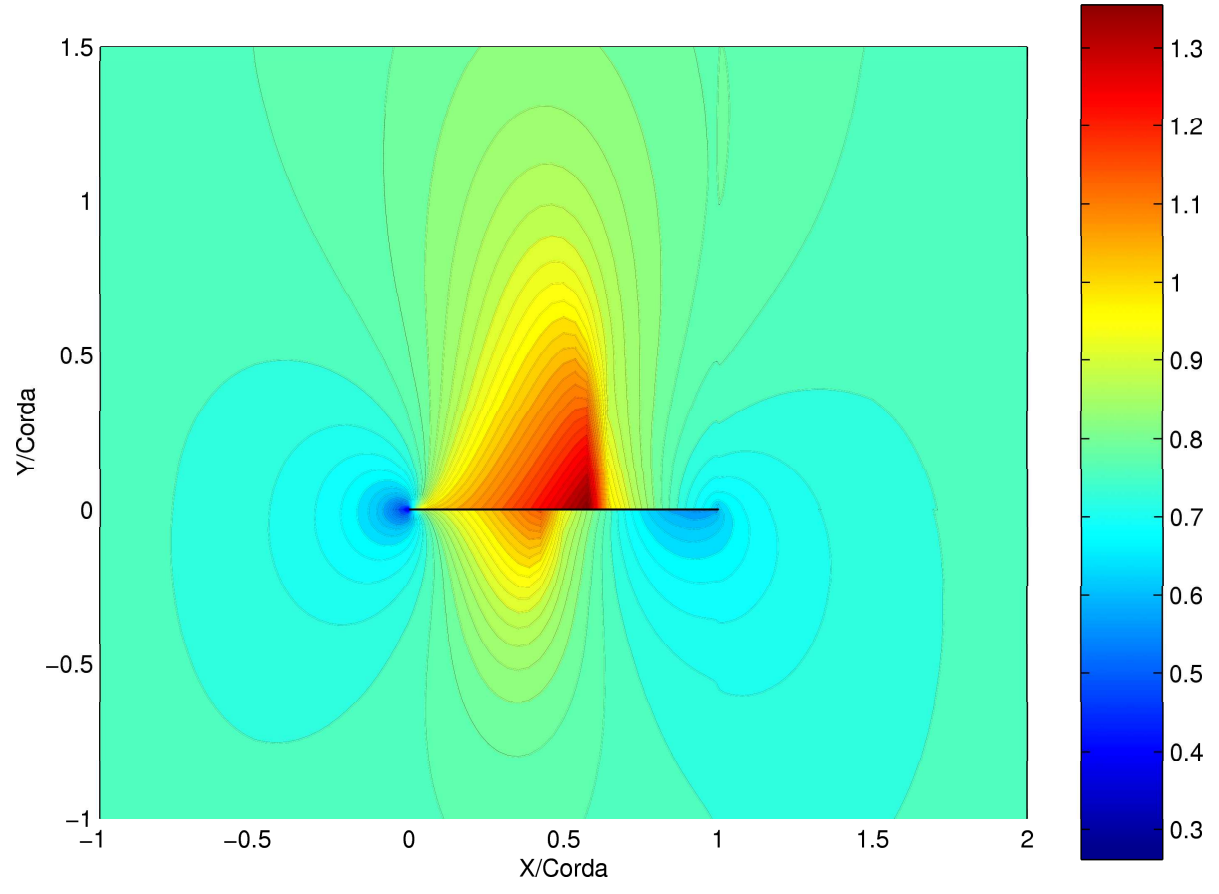

Figura 3.38: Gráfico do número de Mach em torno do perfil NLR7301 com $M_{\infty}=0,752$, $\alpha=0,37^{\circ}$, usando TSD+Ent+Vort+CL.
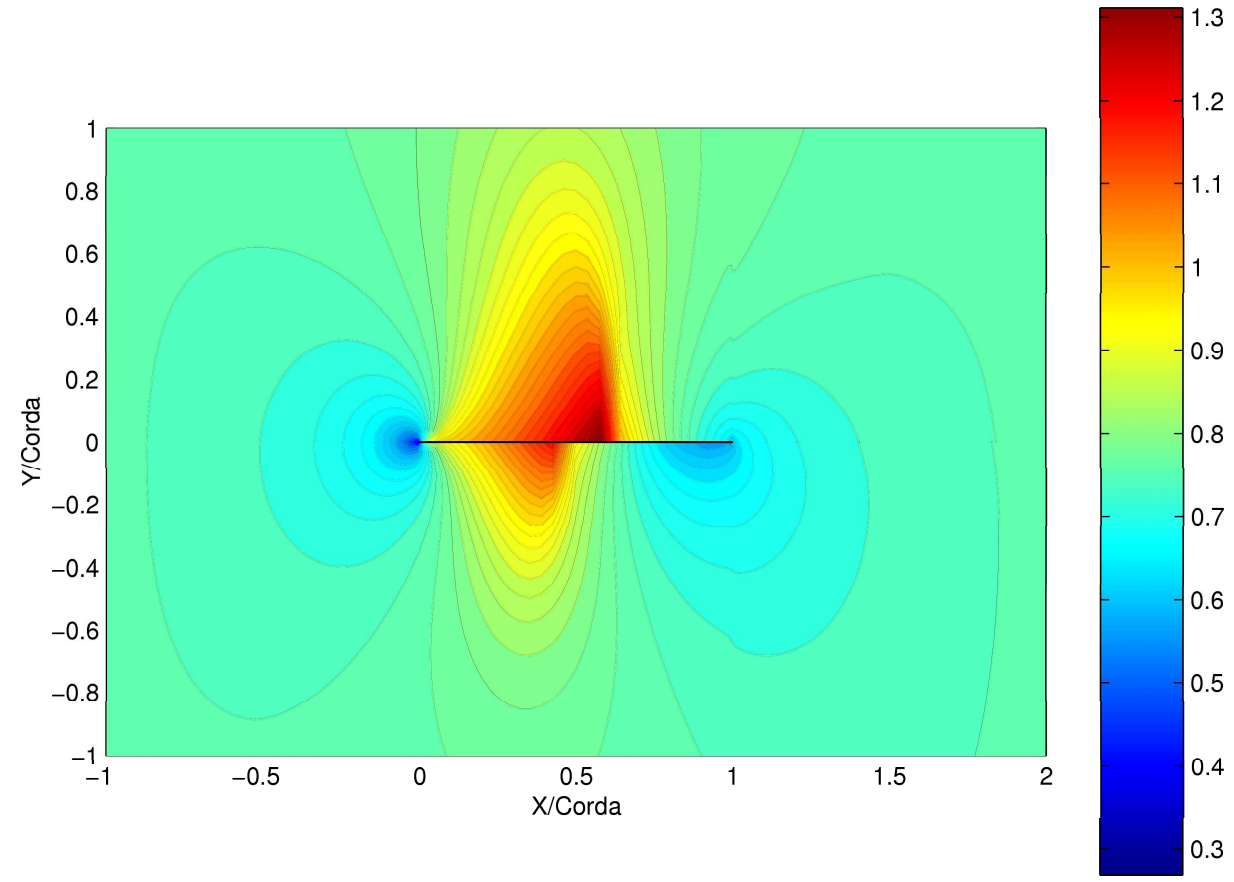

Figura 3.39: Gráfico do número de Mach em torno do perfil NLR7301 $\mathrm{com} M_{\infty}=0,753$, $\alpha=-0,08^{\circ}$, usando TSD+Ent+Vort+CL. 


\section{Capítulo 4}

\section{Programa UsTSD}

O programa UsTSD transônico de pequenas perturbações tridimensional foi desenvolvido na Universidade de Kansas. Esse código é capaz de obter resultados não estacionários no domínio da frequência para configurações completas de aeronaves. Ele foi desenvolvido para estudos em aeroelasticidade e aplicado, com sucesso, à previsão de flutter e oscilações de ciclo limite ${ }^{[16]}$.

No presente trabalho de doutorado foi introduzido no programa UsTSD a correção viscosa em perfis de aerofólios usando o método descrito no Capítulo 2, só que como o programa é tridimensional, a correção é feita em cada seção da asa.

Os resultados a serem apresentados neste capítulo são referentes ao programa UsTSD com correção viscosa somente para o caso estacionário e não estacionário. Sendo que nos casos de solução bidimensional foram obtidas usando uma asa retangular de grande alongamento (igual a 40) e tomando os resultados no plano de simetria, buscando uma condição mais próxima da bidimensional verdadeira.

\subsection{Critério de Convergência do Programa UsTSD}

O critério de parada adotado para gerar os resultados deste capítulo é idêntico ao adotado para os programas TSD+Ent+Vort e TSD+Ent+Vort+CL apresentado no Capítulo 3, que é a convergência quando o resíduo máximo for menor que $10^{-7}$ ou a parada na iteração 2000 . Em todos os casos analisados, o resíduo ficou abaixo da ordem de $10^{-5}$ antes das 2000 iterações. Pode-se observar na Figura 4.1 um exemplo de evolução do resíduo. 


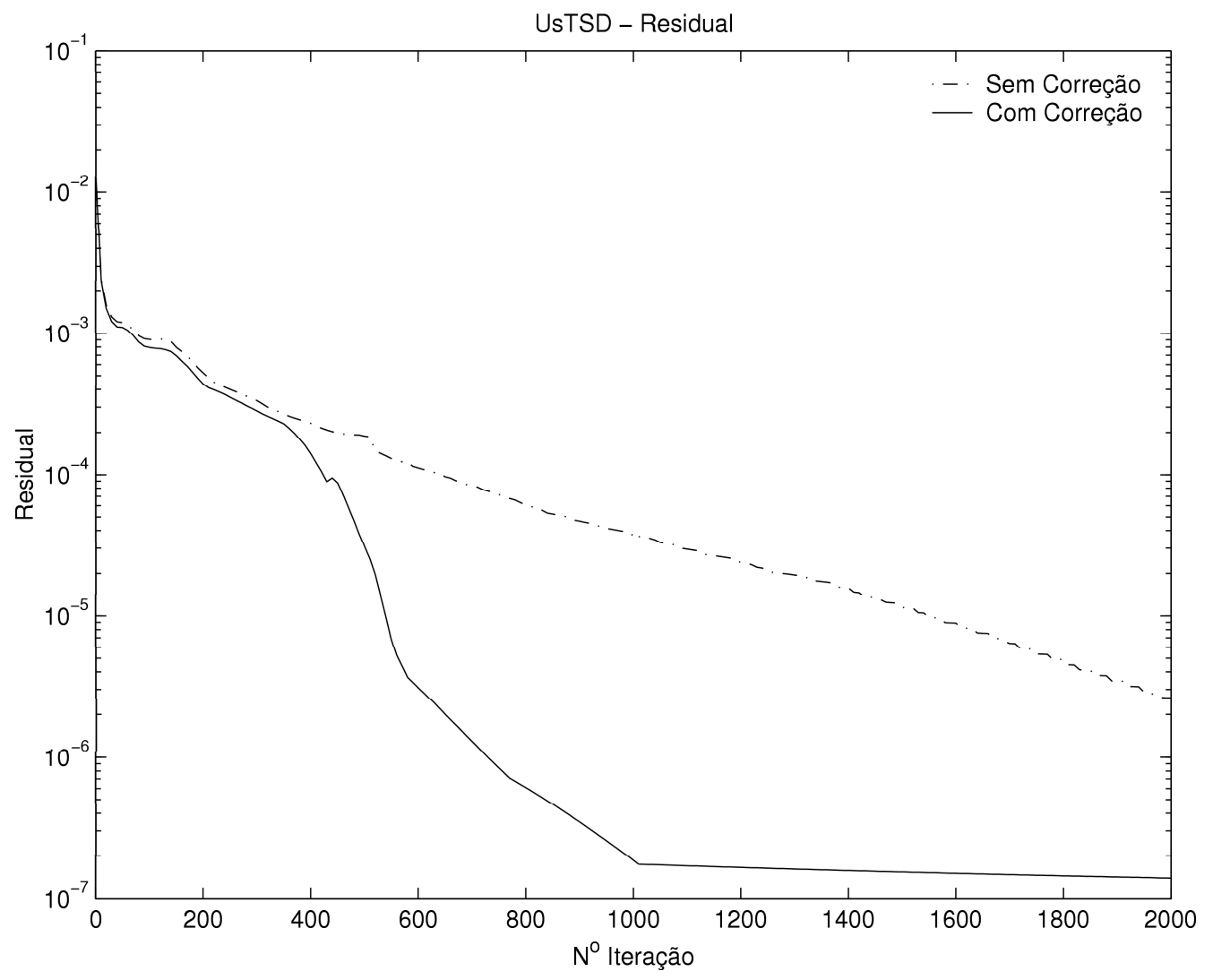

Figura 4.1: Resíduo do programa UsTSD aplicado na seção RAE2822 com $M_{\infty}=0,730$, $\alpha=3,19^{\circ}$, sem e com Correção Viscosa.

Caso mudemos o critério de convergência para $10^{-5}$, a correção viscosa acelera a convergência em relação à análise sem correção, como mostra a Figura 4.2. 


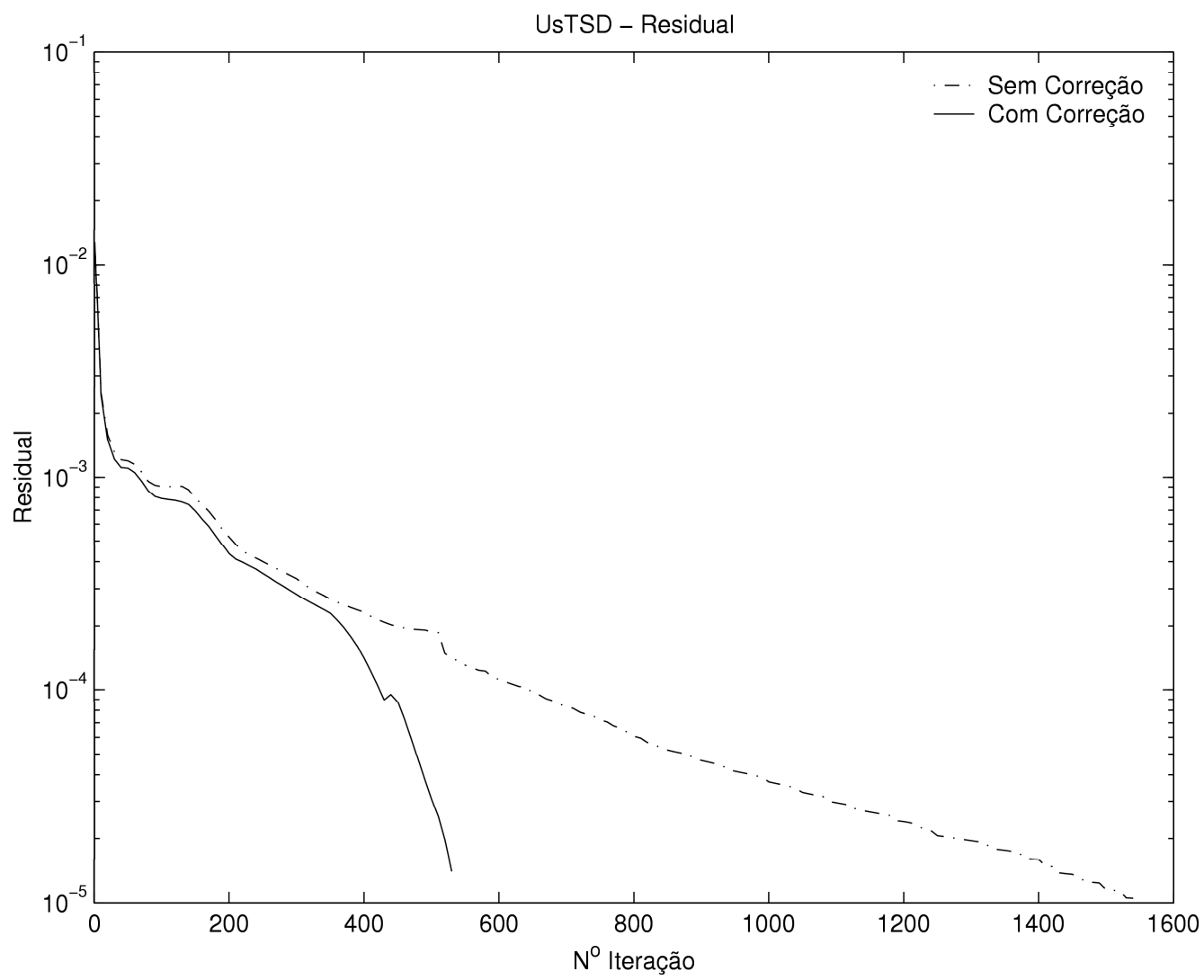

Figura 4.2: Resíduo do programa UsTSD aplicado na seção RAE2822 com $M_{\infty}=0,730$, $\alpha=3,19^{\circ}$, sem e com Correção Viscosa.

Aqui pode-se observar um efeito interessante da correção viscosa, que é a aceleração da convergência como mostram as figuras 4.1 e 4.2. De certa maneira a posição do choque é localizada mais rapidamente, pois o espeçamento da camada limite após o choque empurra o choque a montante, sendo que o choque a cada iteração tende a jusante, assim encontrando um equilíbrio mais rapidamente do que se não tivesse correção viscosa. 


\subsection{Resultados do Programa UsTSD Estacionário}

\subsubsection{Bidimensional}

Os casos a serem apresentados são:

- RAE2822, em

- $M_{\infty}=0,730, \alpha=3,19^{\circ}$ e $\operatorname{Re}=8,90 \times 10^{6}$;

- $M_{\infty}=0,729, \alpha=2,31^{\circ}$ e $\operatorname{Re}=8,90 \times 10^{6}$.

- NACA0012, em

- $M_{\infty}=0,750, \alpha=1,00^{\circ}$ e $\operatorname{Re}=5,30 \times 10^{6}$;

- $M_{\infty}=0,750, \alpha=3,00^{\circ}$ e $\operatorname{Re}=5,30 \times 10^{6}$;

- $M_{\infty}=0,800, \alpha=1,25^{\circ}$ e $\operatorname{Re}=5,30 \times 10^{6}$.

- NRL7301, em

- $M_{\infty}=0,752, \alpha=0,37^{\circ}$ e $\operatorname{Re}=8,20 \times 10^{6}$;

- $M_{\infty}=0,753, \alpha=-0,08^{\circ}$ e $\operatorname{Re}=8,20 \times 10^{6}$.

\section{RAE2822}

A Figura 4.3 mostra a espessura de deslocamento da seção RAE2822 com $M_{\infty}=0,730$, $\alpha=3,19^{\circ}$ e com $M_{\infty}=0,729, \alpha=2,31^{\circ}$, ambos com $\operatorname{Re}=8,90 \times 10^{6}$ e usando UsTSD com correção viscosa. Pode-se observar que os resultados apresentados pela Figura 4.3 são semelhantes aos apresentados no Capítulo 3, porém o gradiente de espessamento logo após o choque no extradorso é mais intenso, quando se compara com a Figura 4.4 (que corresponde a Figura 3.12). Uma das prováveis causas desta diferença é devido ao código TSD usar as equações na forma não conservativas e o código UsTSD usar na forma conservativa ${ }^{[3]}$. 


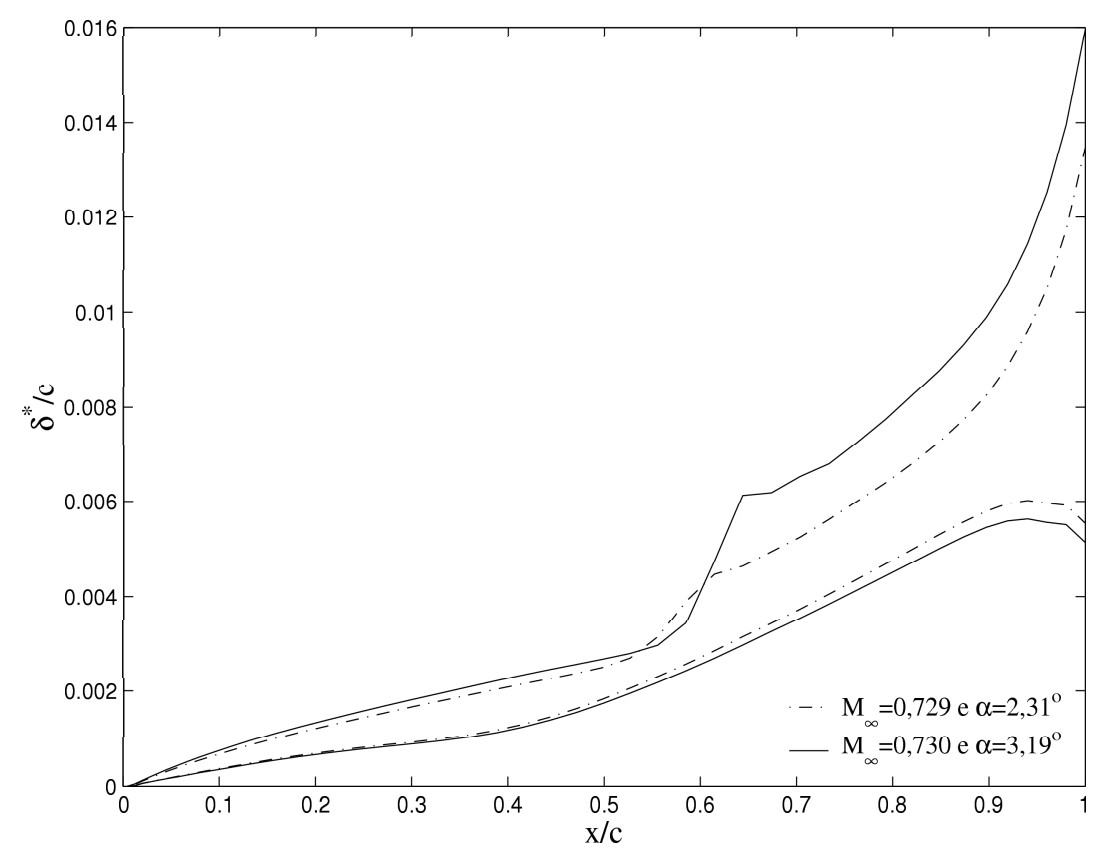

Figura 4.3: Comparação da espessura de deslocamento para a seção RAE2822 com $M_{\infty}=0,730, \alpha=3,19^{\circ}$ e com $M_{\infty}=0,729, \alpha=2,31^{\circ}$, usando UsTSD.

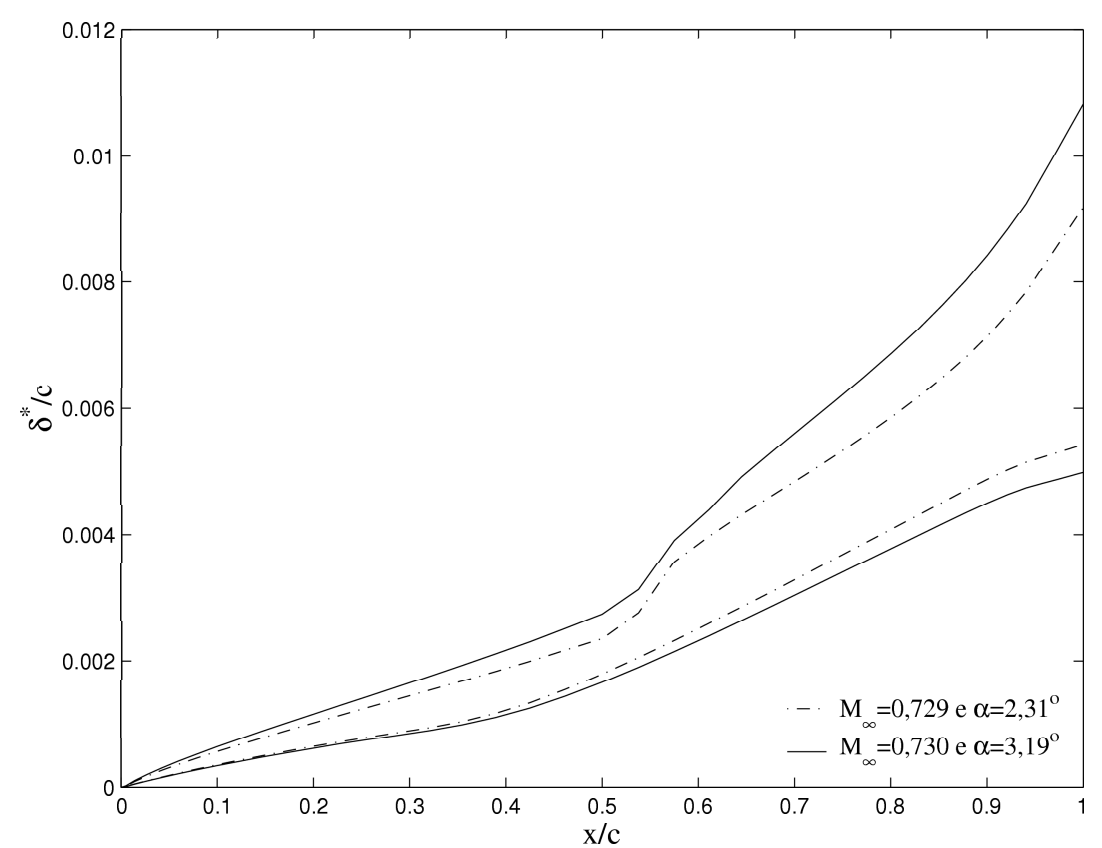

Figura 4.4: Comparação da espessura de deslocamento para a seção do perfil RAE2822 com $M_{\infty}=0,730, \alpha=3,19^{\circ}$ e com $M_{\infty}=0,729, \alpha=2,31^{\circ}$, usando o programa TSD. (Corresponde a Figura 3.12). 
Os resultados obtidos com a correção viscosa apresentaram melhoras consideráveis, como pode-se ver nas Figuras 4.5 e 4.6. A distribuição de presssão obtida pela aplicação da correção viscosa apresentou uma proximidade muito grande com os resultados experimentais ${ }^{[22]}$. Além disso, os efeitos que são apresentados pela correção viscosa, como o de deslocar a posição do choque a montante e o de reduzir a intensidade do choque, são muito mais visíveis, pois o programa TSD as equações estava na forma não conservativa e o programa UsTSD está na forma conservativa, quando comparamos as Figuras 4.5 e $4.6 \mathrm{com}$ as figuras as Figuras 4.7 e 4.8 respectivamente. 


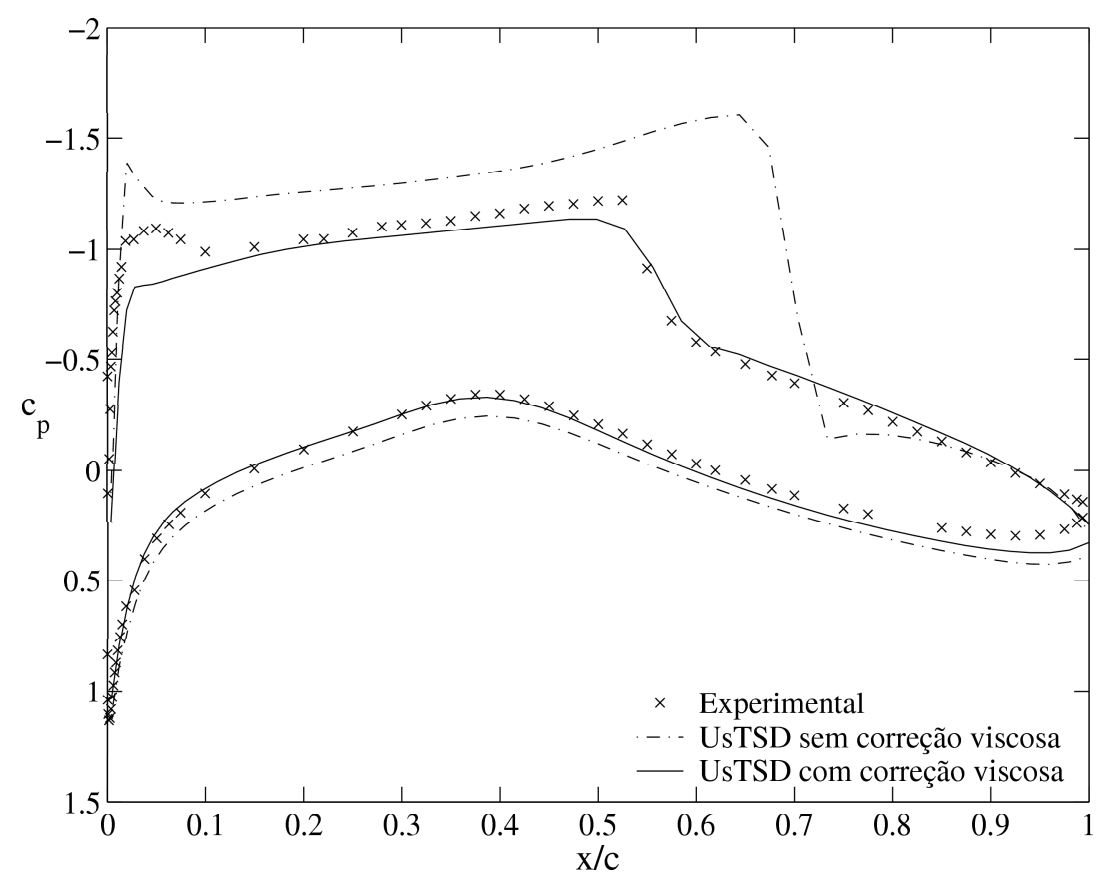

Figura 4.5: Distribuição do coeficiente de pressão para a seção RAE2822 com $M_{\infty}=0,730$, $\alpha=3,19^{\circ}$, usando UsTSD sem e com Correção Viscosa.

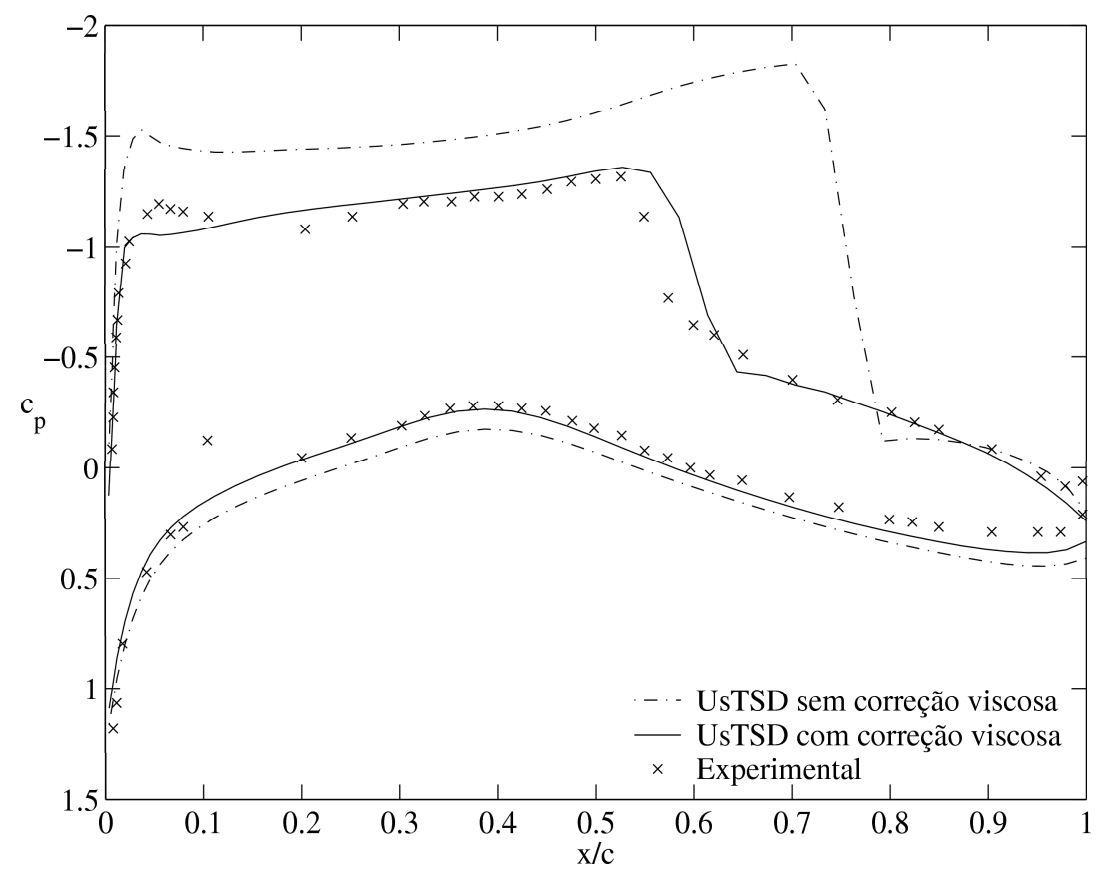

Figura 4.6: Distribuição do coeficiente de pressão para a seção RAE2822 $\operatorname{com} M_{\infty}=0,729$, $\alpha=2,31^{\circ}$, usando UsTSD sem e com Correção Viscosa. 


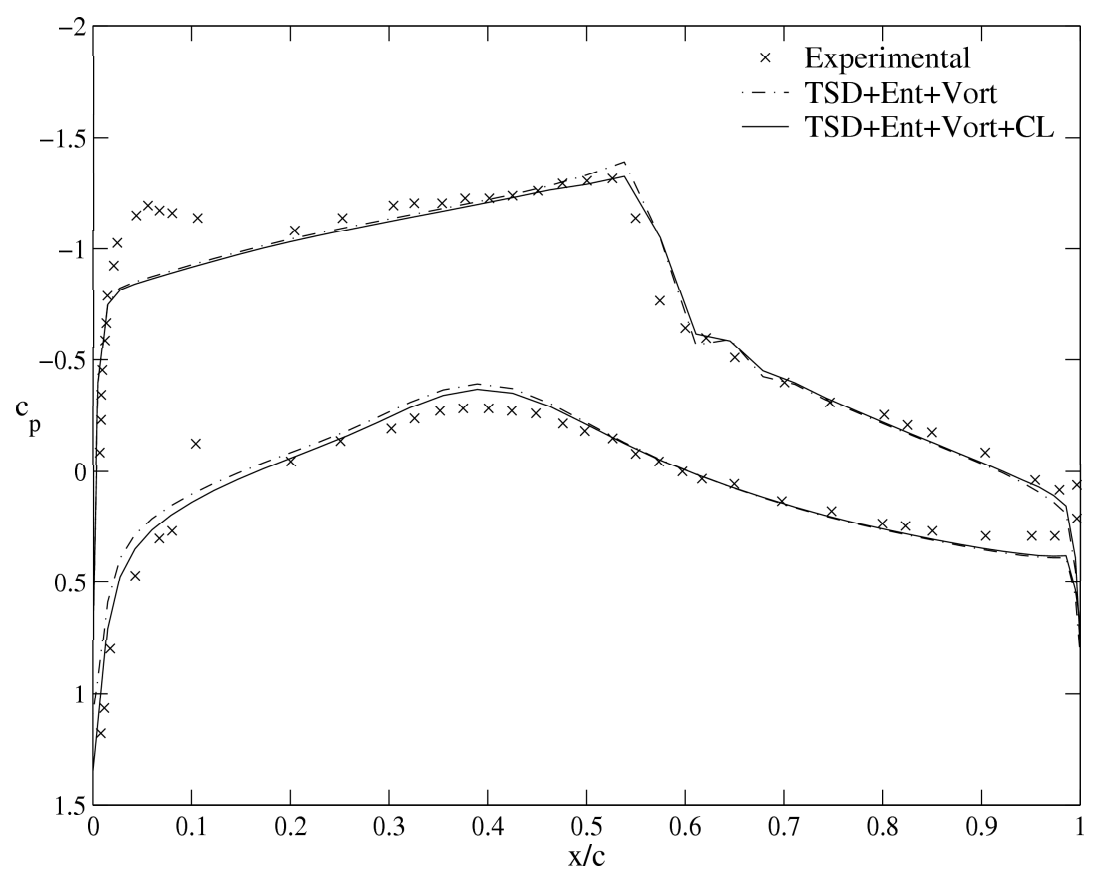

Figura 4.7: Distribuição do coeficiente de pressão para a seção do perfil RAE2822 com $M_{\infty}=0,730$ e $\alpha=3,1^{\circ}$, usando TSD sem e com Correção Viscosa.(Corresponde a Figura $3.8)$.

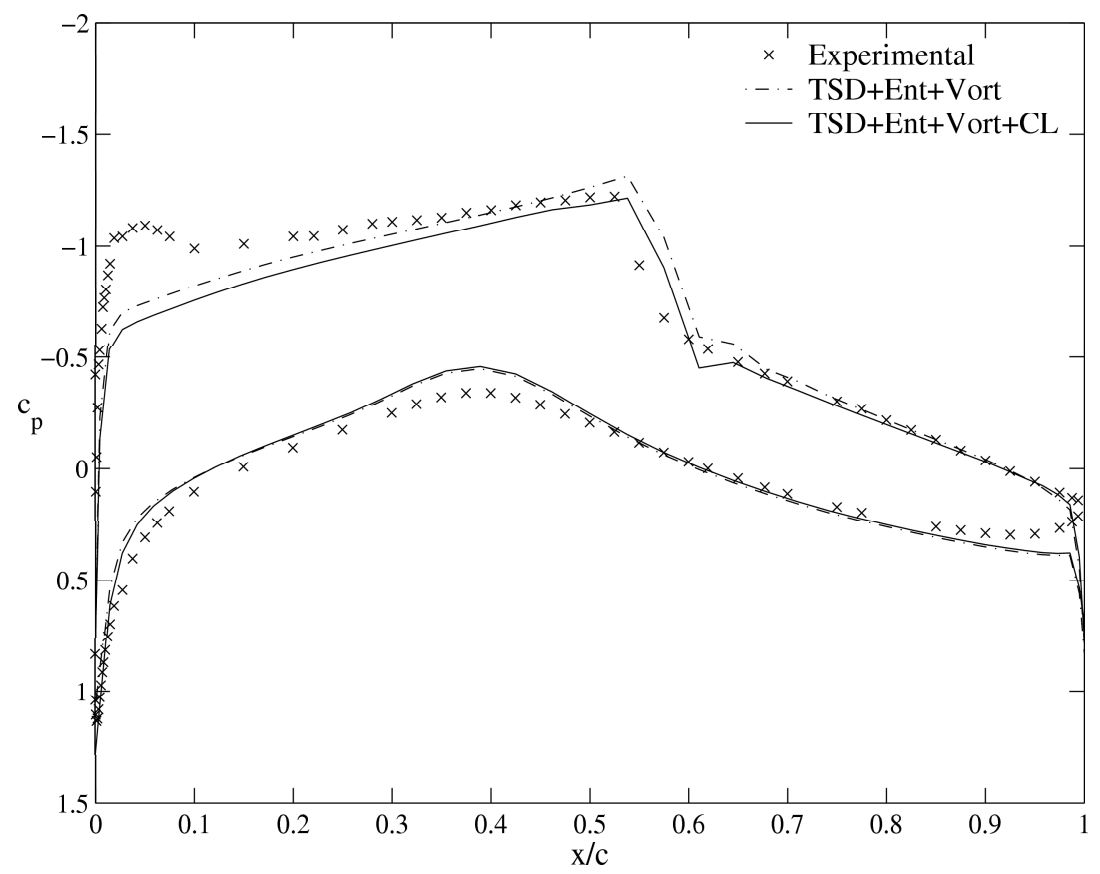

Figura 4.8: Distribuição do coeficiente de pressão para a seção do perfil RAE2822 com $M_{\infty}=0,729$ e $\alpha=2,31^{\circ}$, usando TSD sem e com Correção Viscosa.(Corresponde a Figura $3.9)$. 


\section{NACA0012}

Para o perfil NACA0012 foram analisados os casos com o número de Mach de entrada $\left(M_{\infty}\right)$ igual a 0,750 e os ângulos de ataque $(\alpha)$ iguais a $1,00^{\circ}$ e $3,00^{\circ}$ e com o número de Mach de entrada $\left(M_{\infty}\right)$ igual a 0,800 e o ângulo de ataque $(\alpha)$ igual a $1,25^{\circ}$, sendo que os dois primeiros casos foram comparados com dados experimentais ${ }^{[35}$. e o último com dados computacionais obtidos pelo programa FLO52 ${ }^{[5]}$. Lembrando que o número de Reynols usado para estes casos é $\mathrm{Re}=5,30 \times 10^{6}$.

Para os casos com número de Mach de entrada $\left(M_{\infty}\right)$ igual a 0,750 e os ângulos de ataque $(\alpha)$ iguais a $1,00^{\circ}$ e $3,00^{\circ}$, observou-se que com uma pequena alteração no ângulo de ataque a itensidade do choque mudou significativamente, como mostram as Figuras 4.9 e 4.10. Conseqüentemente a espessura de deslocamento também sofreu uma variação bastante significativa, como mostra a Figura 4.11. A correção viscosa aproxima os resultados numérico e experimental mas com correlação pior que aquela obtida para o RAE2822.

Estes mesmos fenômenos foram vistos quando usou-se o programa TSD no Capítulo 3.

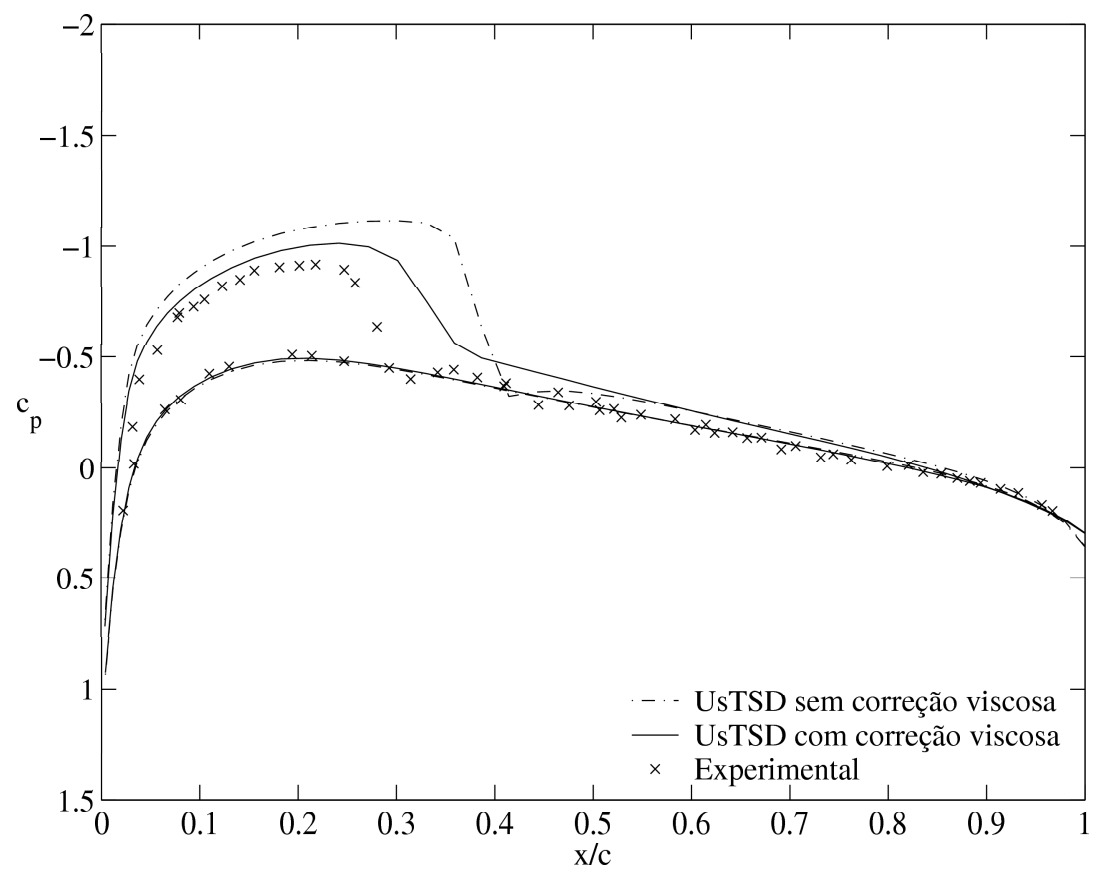

Figura 4.9: Distribuição do coeficiente de pressão para a seção NACA0012 $\operatorname{com} M_{\infty}=0,750$, $\alpha=1,00^{\circ}$, usando UsTSD sem e com Correção Viscosa.

O caso com número de Mach de entrada $\left(M_{\infty}\right)$ igual a 0,800 e o ângulo de ataque $(\alpha)$ igual a $1,25^{\circ}$, é comparado com dados computacionais obtidos pelo programa FLO52 ${ }^{[5]}$. 


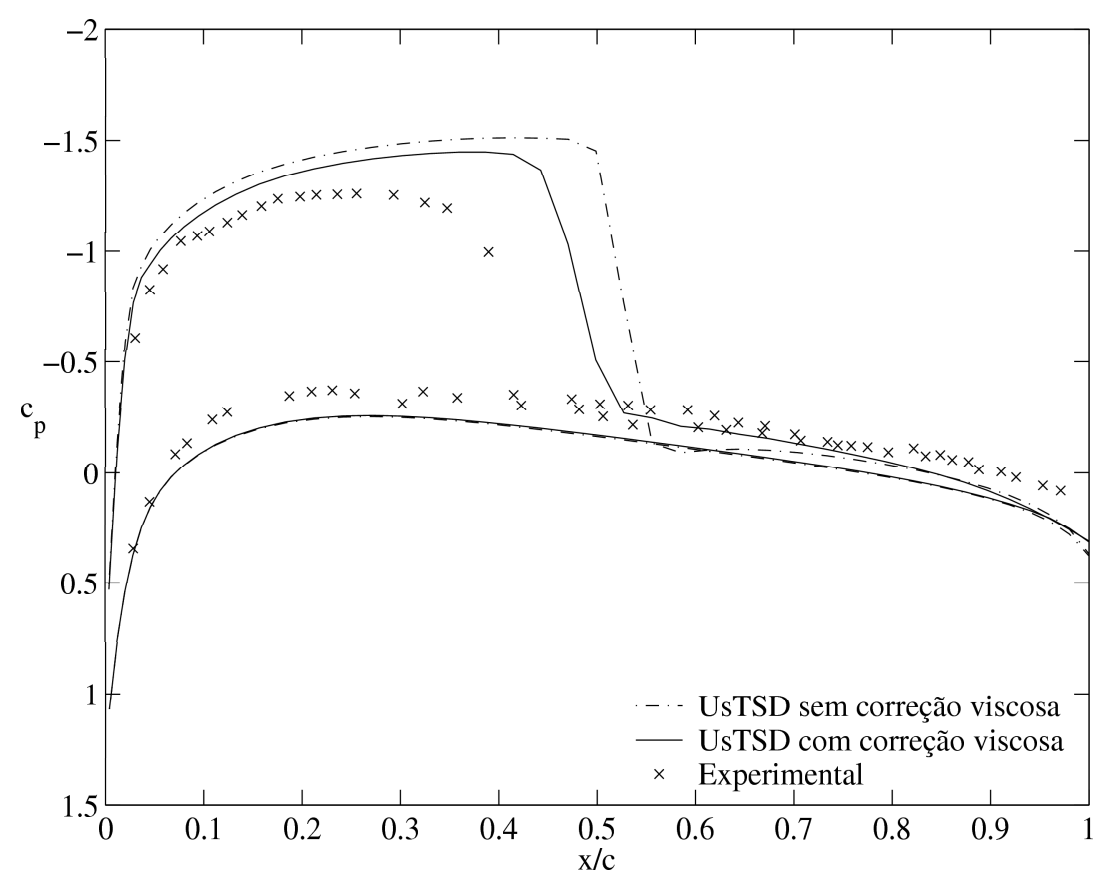

Figura 4.10: Distribuição do coeficiente de pressão para a seção NACA0012 $\mathrm{com} M_{\infty}=$ 0,750, $\alpha=3,00^{\circ}$, usando UsTSD sem e com Correção Viscosa.

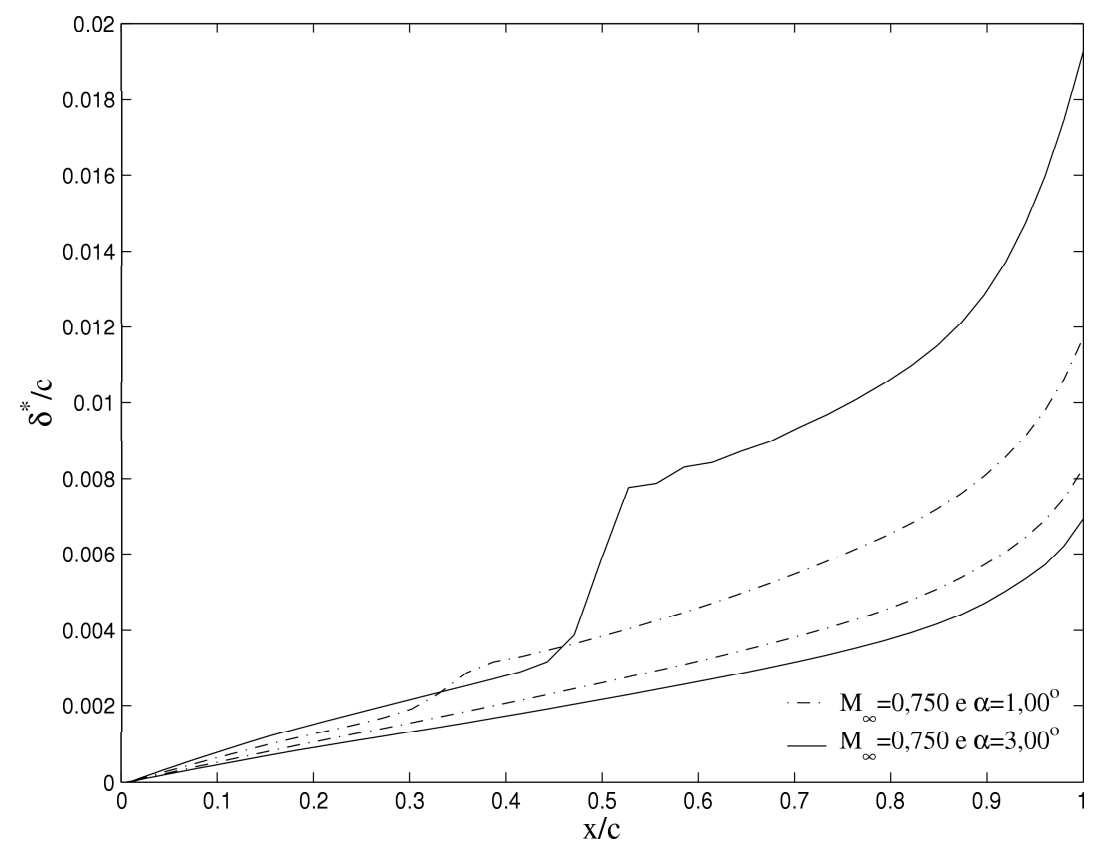

Figura 4.11: Comparação da espessura de deslocamento para a seção NACA0012 $\operatorname{com} M_{\infty}=$ $0,750, \alpha=1,00^{\circ}$ e $\operatorname{com} M_{\infty}=0,750, \alpha=3,00^{\circ}$, usando UsTSD com Correção Viscosa. 


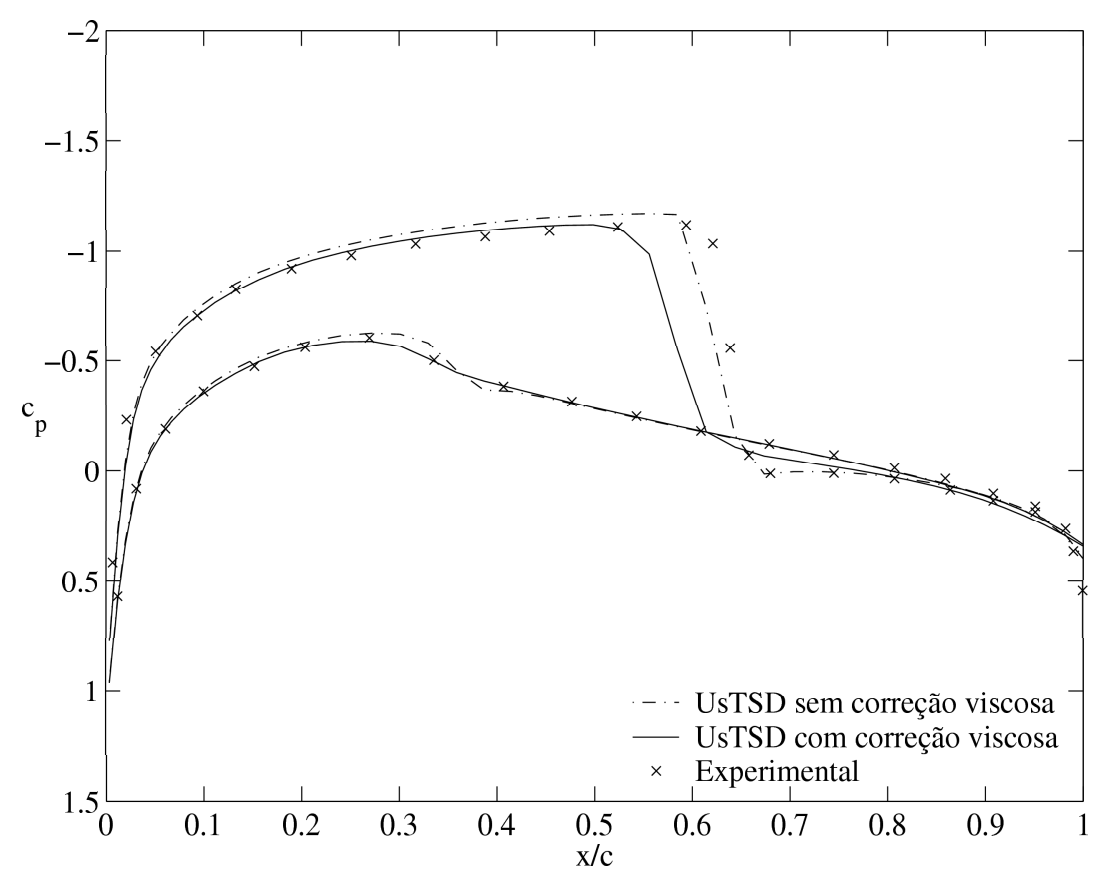

Figura 4.12: Distribuição do coeficiente de pressão para a seção NACA0012 $\mathrm{com} M_{\infty}=$ $0,800, \alpha=1,25^{\circ}$, usando UsTSD sem e com Correção Viscosa e do programa FLO52 ${ }^{[5]}$.

Como pode-se observar na Figura 4.12 o efeito da correção viscosa é o esperado levando em conta que o FLO52 é um código invíscido.

\section{NRL7301}

Da mesma forma que anteriormente, também foi feita a análise para o perfil NRL7301. Neste caso observa-se que nem sempre a espessura de deslocamento é estritamente crescente, nas Figuras 4.13 e 4.14 pode-se observar que a espessura de deslocamento no intradorso perto do bordo de fuga torna-se decrescente. O mesmo fenômeno ocorreu quando usou-se o programa TSD. 


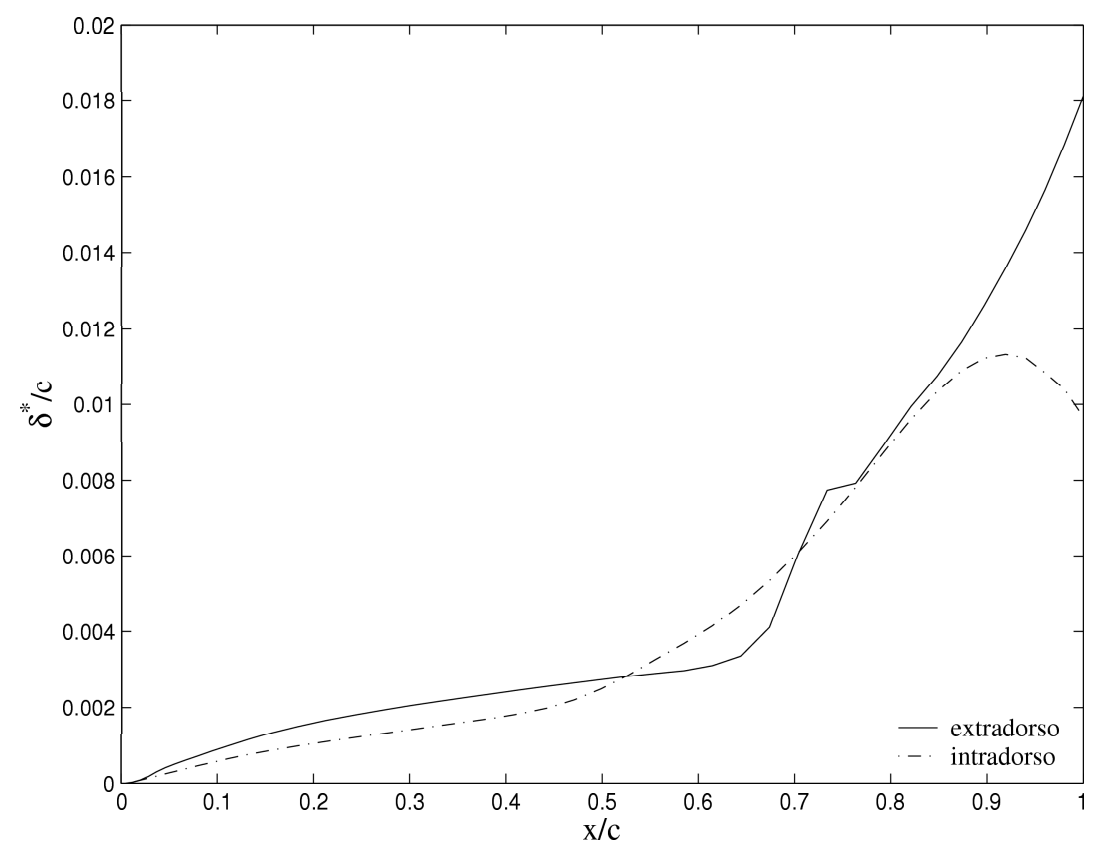

Figura 4.13: Comparação da espessura de deslocamento para a seção NRL7301 com $M_{\infty}=0,752, \alpha=0,37^{\circ}$, usando UsTSD sem e com Correção Viscosa.

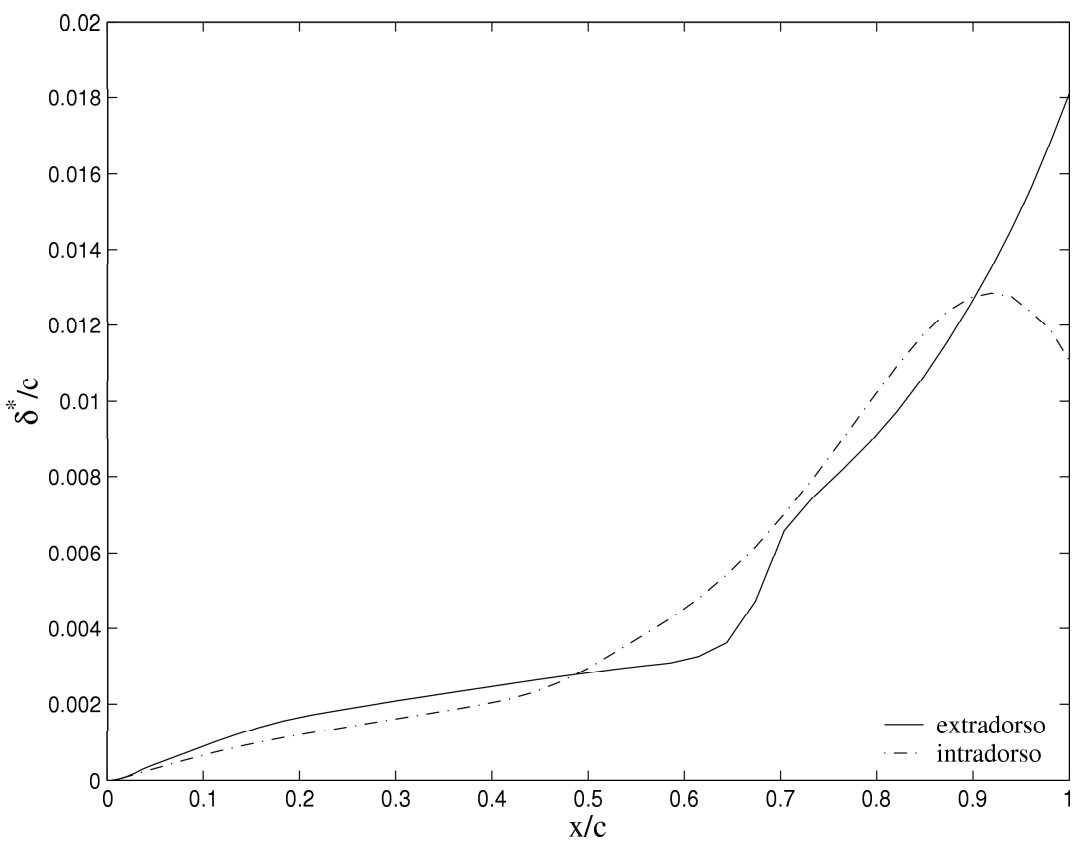

Figura 4.14: Comparação da espessura de deslocamento para a seção NRL7301 com $M_{\infty}=0,753, \alpha=-0,08^{\circ}$, usando UsTSD sem e com Correção Viscosa. 
Nas Figuras 4.15 e 4.16 pode-se perceber diferenças entre os dados simulados e os dados experimentais, especialmente na região do choque a na região próxima ao bordo de ataque onde o resultado numérico apresenta um pico de sucção bastante pronunciado. Isto ocorre porque o raio do bordo de ataque é bastante grande quando comparado com os outros perfis analisados. O efeito da correção da viscosidade aproxima os resultados na região do choque e reduz o pico de sucção. Neste caso, no entanto, a correção viscosa não é suficiente para gerar uma boa correlação com os resultados experimentais.

Quando compara-se os resultados obtidos no Capítulo 3, há uma diferênça bem visível na distribuição de pressão próximo ao bordo de ataque quando utiliza-se o programa UsTSD e o TSD como pode-se observar quando se compara as Figuras 4.15 e $4.16 \mathrm{com}$ as Figuras 4.17 e 4.18 respectivamente.

Os possíveis fatores identificados para estas diferenças foram:

- o programa TSD usa a equação na forma não consevativa e o UsTSD na forma conservativa;

- o perfil NRL7301 possi um raio muito grande no bordo de ataque o que torna a simulação numérica mais complexa, especialmente devido à hipótese de pequenas perturbações;

- o pico de sucção no código UsTSD é característico de códigos invíscidos. Este tipo de resultado foi observado em testes com o programa XFoil. A solução invíscida apresentava um pico de sucção que ficava reduzido quando era usada a opção de correção viscosa. A viscosidade reduz a aceleração do escoamento no bordo de ataque reduzindo assim o pico de sucção. 


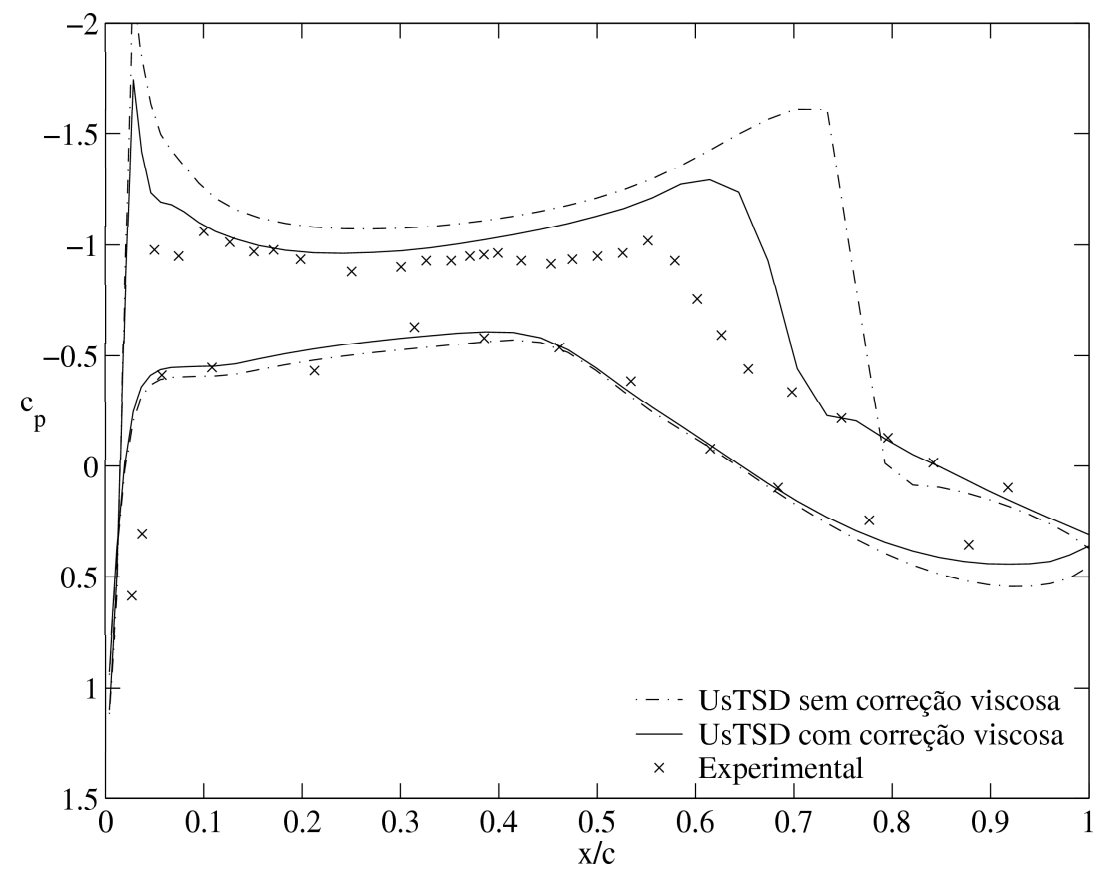

Figura 4.15: Distribuição do coeficiente de pressão para a seção NRL7301 com $M_{\infty}=0,752$, $\alpha=0,37^{\circ}$, usando UsTSD sem e com Correção Viscosa.

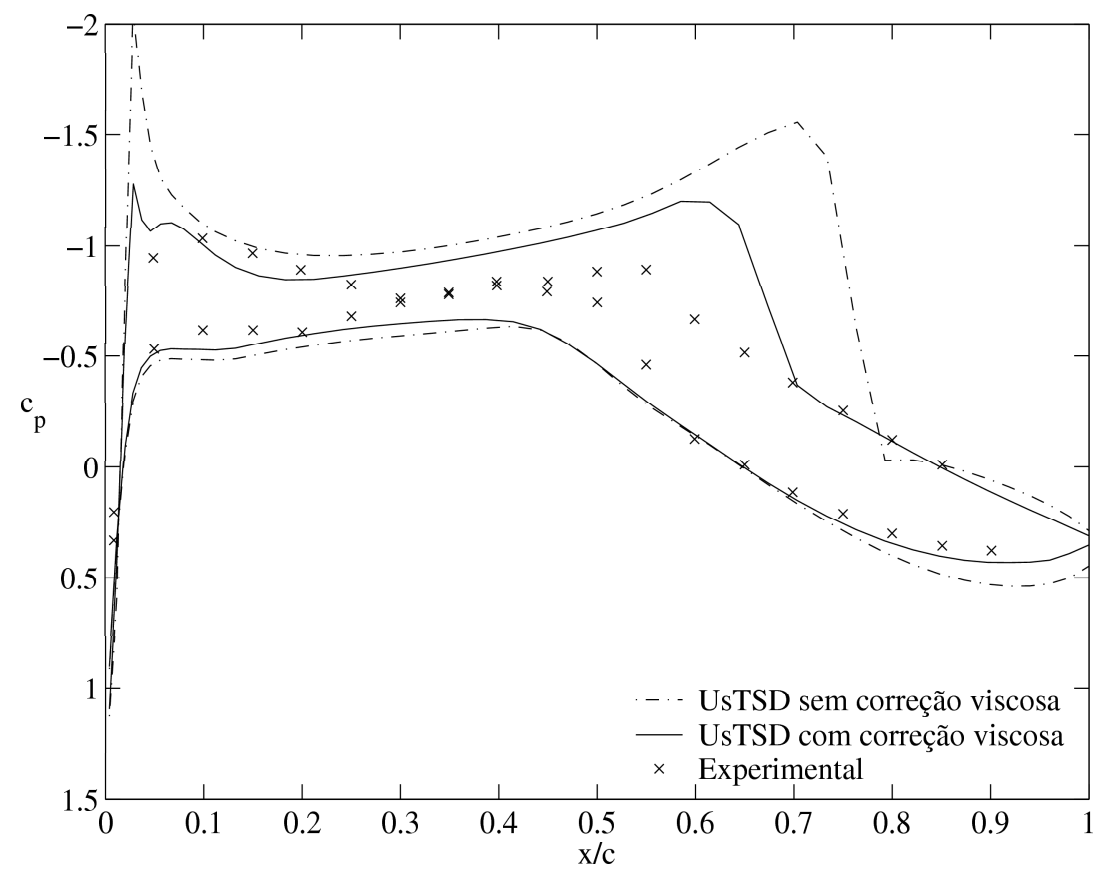

Figura 4.16: Distribuição do coeficiente de pressão para a seção NRL7301 com $M_{\infty}=0,753$, $\alpha=-0,08^{\circ}$, usando UsTSD sem e com Correção Viscosa. 


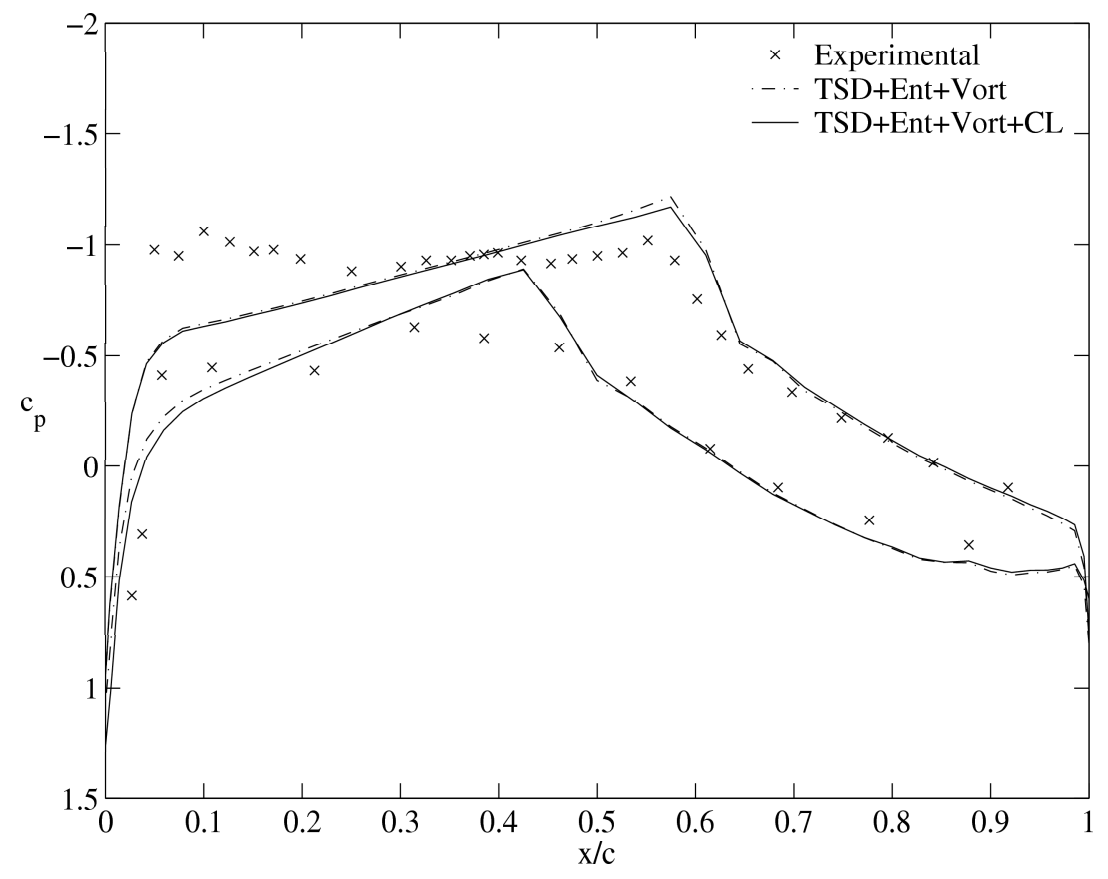

Figura 4.17: Coeficiente de pressão para a seção do perfil NLR7301 com $M_{\infty}=0,752$, $\alpha=0,37^{\circ}$, usando TSD sem e com Correção Viscosa. (Corresponde a Figura 3.34)

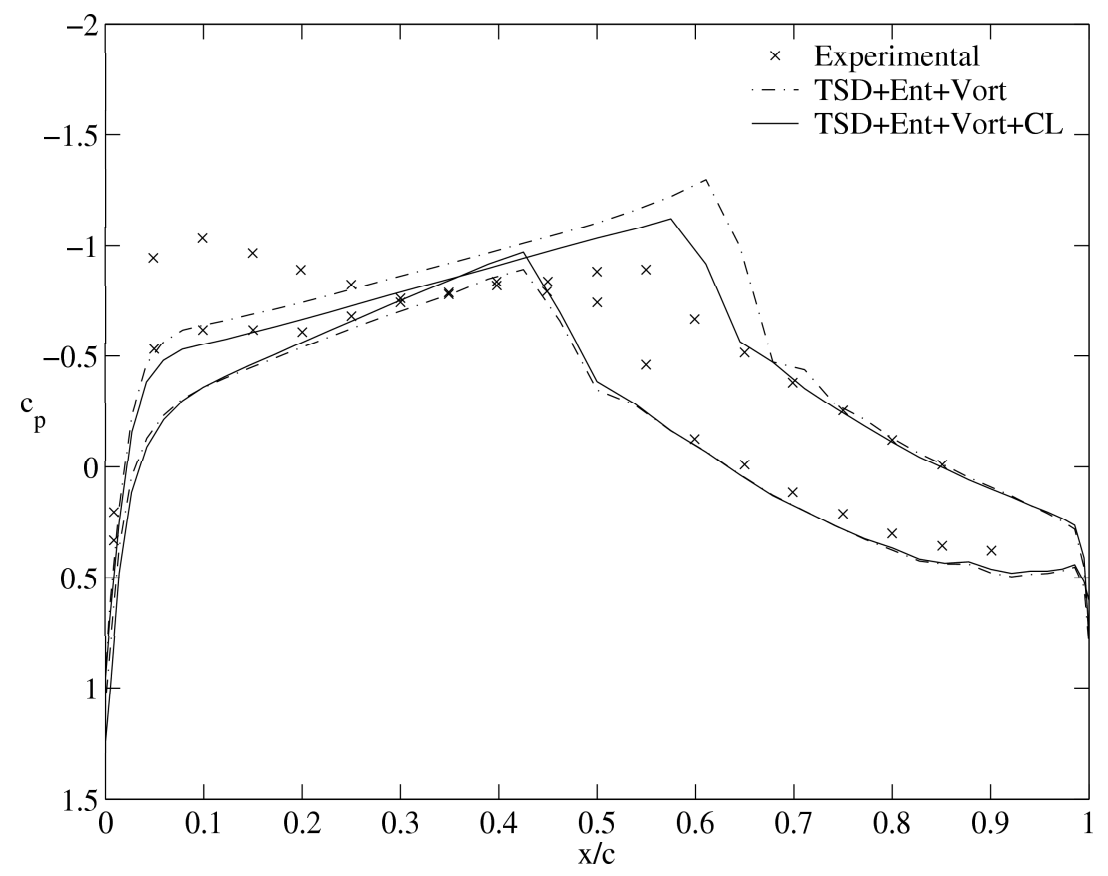

Figura 4.18: Coeficiente de pressão para a seção do perfil NLR7301 com $M_{\infty}=0,753$, $\alpha=-0,08^{\circ}$, usando TSD sem e com Correção Viscosa. (Corresponde a Figura 3.35) 


\subsubsection{Tridimensional}

As análies tridimensionais para os casos estacionários e não estacionários se baseiam no perfil NRL F5, um perfil modificado do NACA 65-A-004.8. Os dados experimentais para comparação foram obtidos de Geurts ${ }^{[14]}$, usando a seguinte distribuição de orifícios de tomada de pressão e de transdutores como mostra a Figura 4.19.

Os casos analisados são:

1. Mach 0,597 , ângulo de ataque $=0,493^{\circ} \mathrm{com} R e=4,77 \times 10^{6}$;

2. Mach 0,896, ângulo de ataque $=0,497^{\circ} \mathrm{com} R e=5,79 \times 10^{6}$;

3. Mach 0,946, ângulo de ataque $=-0,004^{\circ} \operatorname{com} R e=5,89 \times 10^{6}$.

\section{Caso 1}

Pode-se observar que em Mach 0,597, com ângulo de ataque $0,493^{\circ}$ e $R e=4,77 \times 10^{6}$, tem-se que a distribuição de pressão nas seções analisadas apresentam uma boa correlação com os dados experimentais, principalmente nas seções intermediárias (2 a 6) como mostram as Figuras 4.20 e 4.21.

Lembrando que o escoamento em Mach 0,597, o perfil NRL F5 está em condições subsônicas, isto é, não apresentam ondas de choque no perfil. Portanto os resultados obtidos pelo UsTSD sem e com correção viscosa estão muito próximas, como mostram as Figuras 4.20 e 4.21 .

\section{Caso 2}

O perfil NRL F5 em Mach 0,896, com ângulo de ataque $0,497^{\circ}$ e $R e=5,79 \times 10^{6}$, já apresenta choques fracos nas seções 7 e 8 nos dados experimentais, como pode-se observar na Figura 4.23. Além disso, os dados obtidos pelo código UsTSD sem e com correção viscosa apresentam melhores correlações nas seções intermediárias (3 a 6), a qual não apresentam a presença de ondas e choque, e na medida que se aproxima da ponta da asa esta correlação piora, como mostram as Figuras 4.22 e 4.23 . 


\section{Caso 3}

Mas ao submetermos o perfil NRL F5 em Mach 0,946, $\alpha=-0,004^{\circ} \operatorname{com} R e=5,89 \times 10^{6}$, obseva-se que próximo a raiz e a ponta da asa os resultados não apesentam boa correlação, como os casos anteriores.

Porém, neste caso pode-se ver que a onda de choque é mais intenso, com isso os resultados do código UsTSD obtidos usando a correção viscosa apresentou uma correlaçao maior ao experimental que o sem correção viscosa, Figuras 4.24 e 4.25 .

O método de correção viscosa é baseada na distribuição de Mach de cada seção da asa no plano de simetria, esta correção apresenta resultados mais significativos quando na seção apresenta ondas de choque.

Aqui pode-se observar que o código UsTSD para o caso estacionário apresenta melhores resultados nas seções intermediárias e que os efeitos próximo a raiz e a ponta da asa são mais difíceis de se obter.

Além disso, os fenômenos vistos para os casos bidimensionais estacionários se repetem na análise tridimensional. 

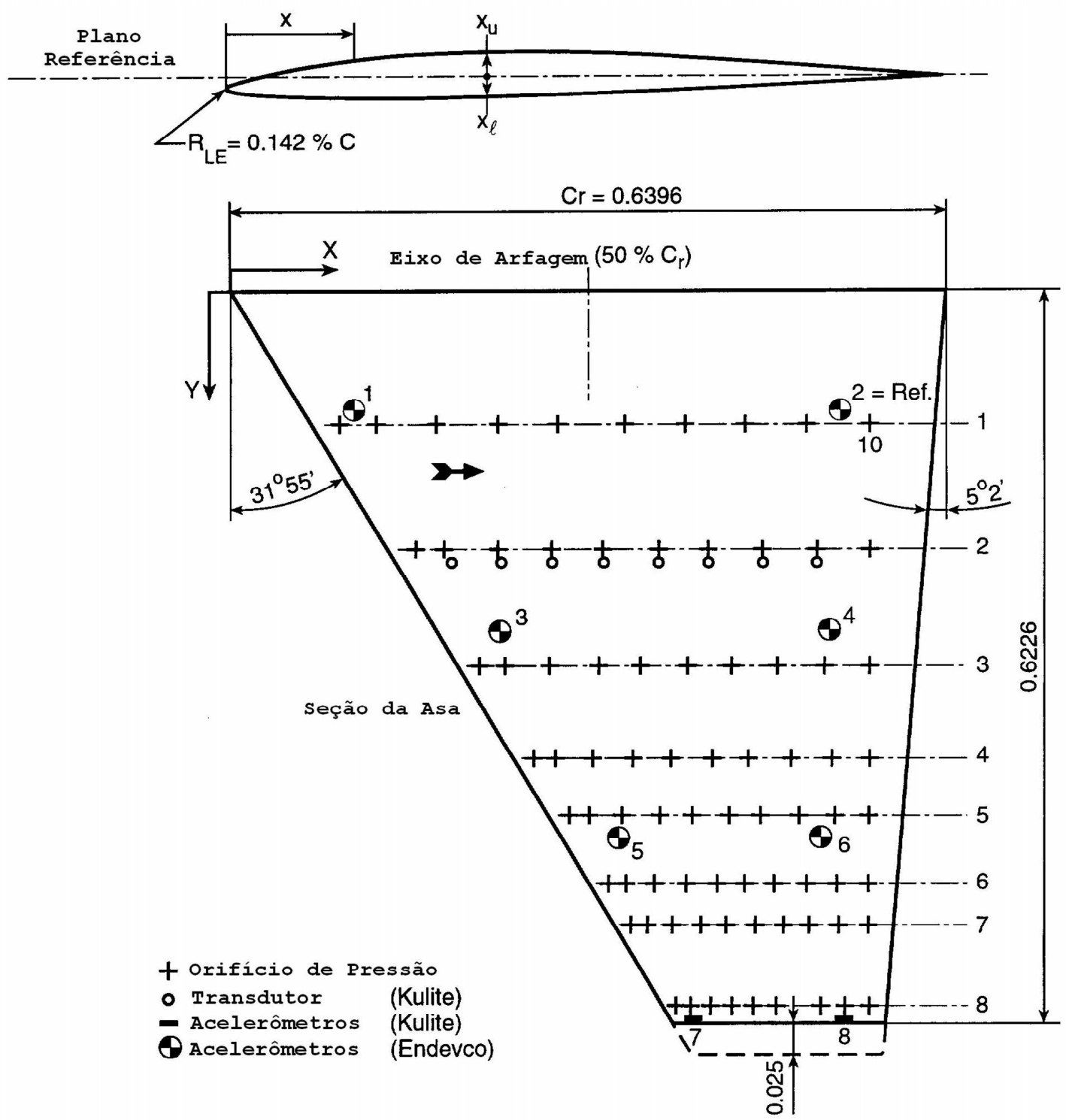

Dimensão em m
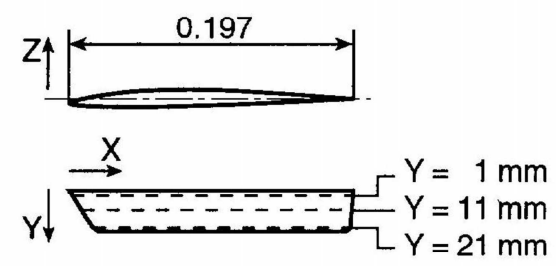

Figura 4.19: NRL F5 com a posição dos orifícios de tomada de pressão e dos transdutores. 

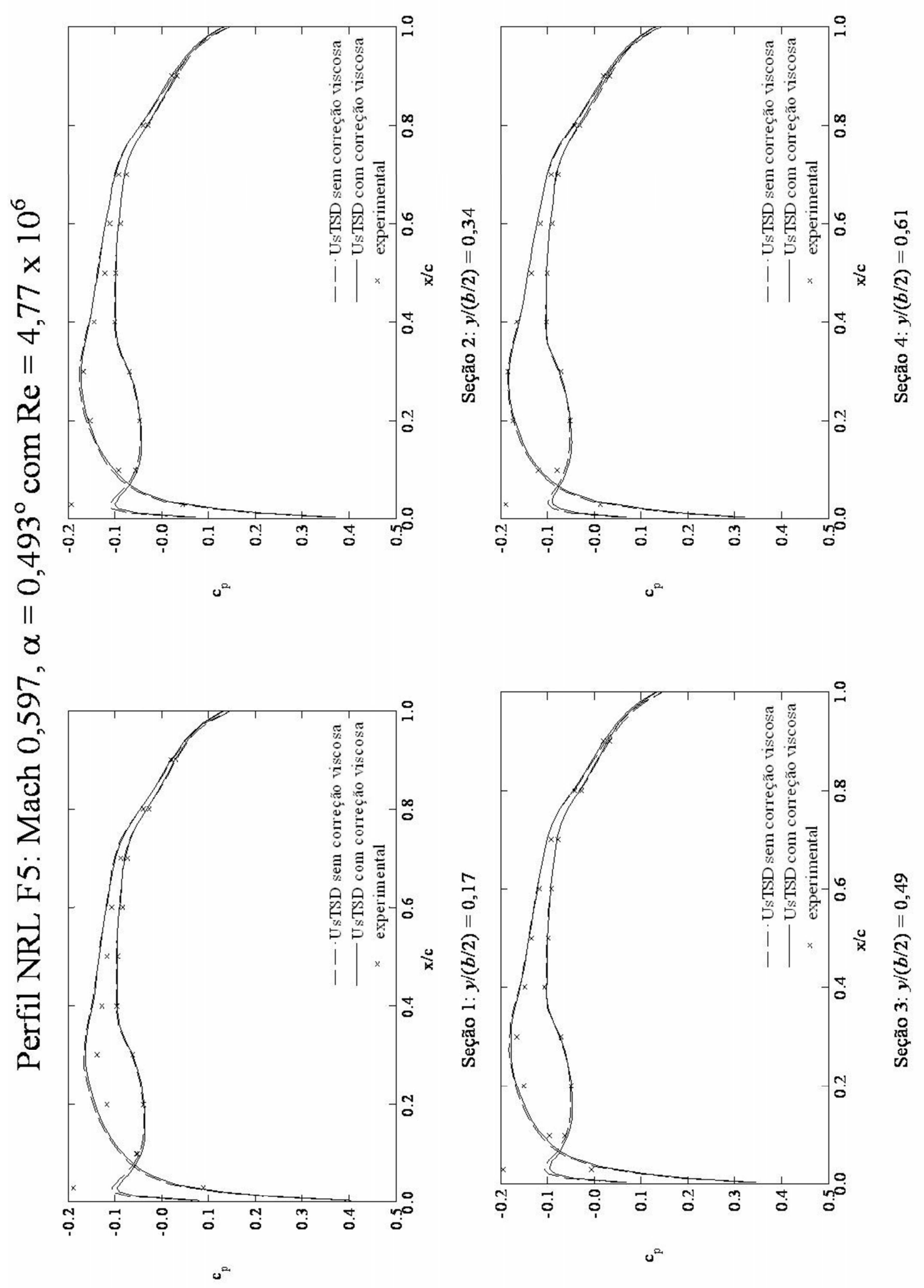

Figura 4.20: NRL F5 em Mach 0,597, $\alpha=0,493^{\circ} \operatorname{com} R e=4,77 \times 10^{6}$ (Parte I). 

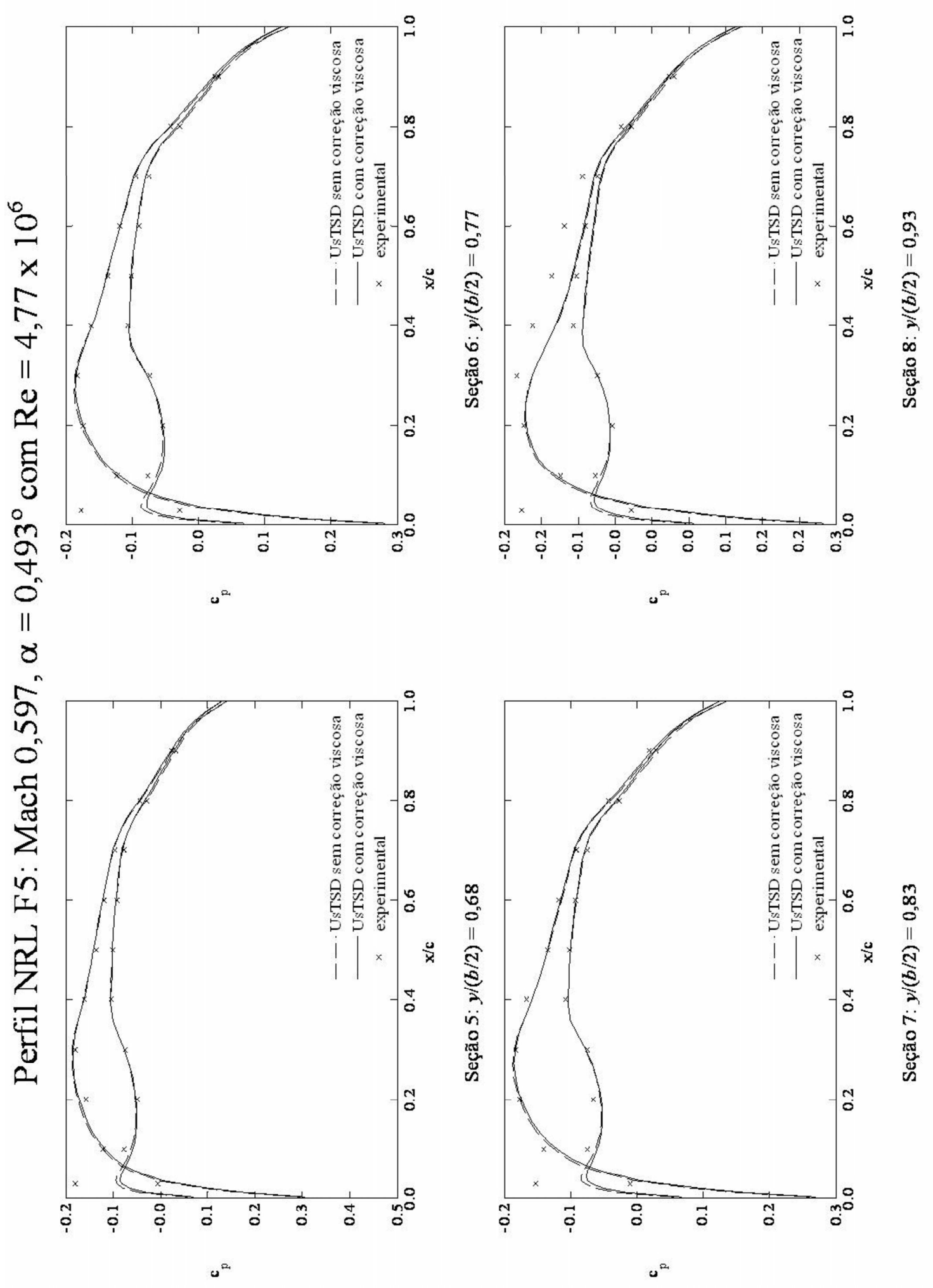

Figura 4.21: NRL F5 em Mach 0,597, $\alpha=0,493^{\circ} \mathrm{com} R e=4,77 \times 10^{6}$ (Parte II). 

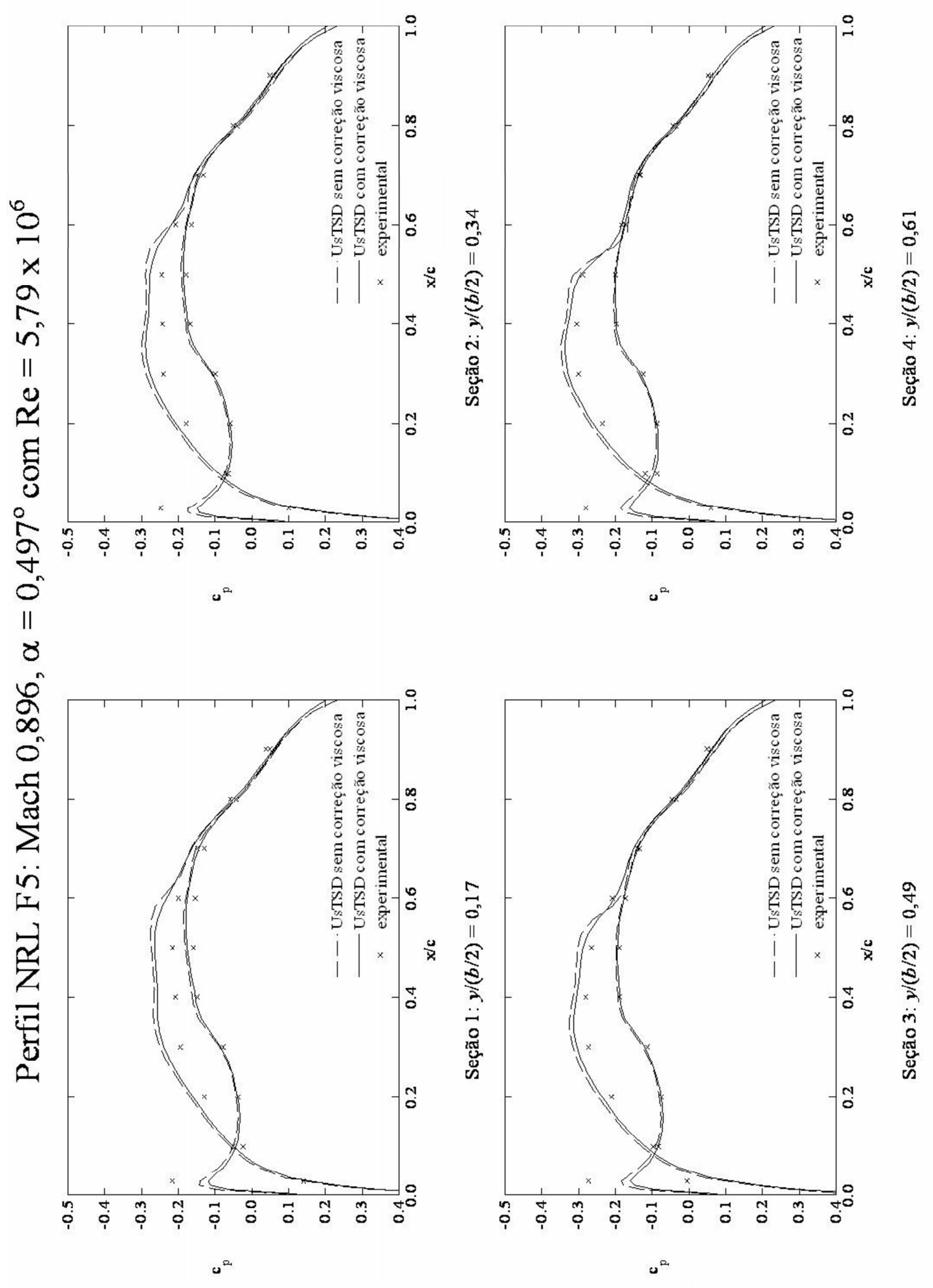

Figura 4.22: NRL F5 em Mach 0,896, $\alpha=0,497^{\circ} \operatorname{com} R e=5,79 \times 10^{6}$ (Parte I). 

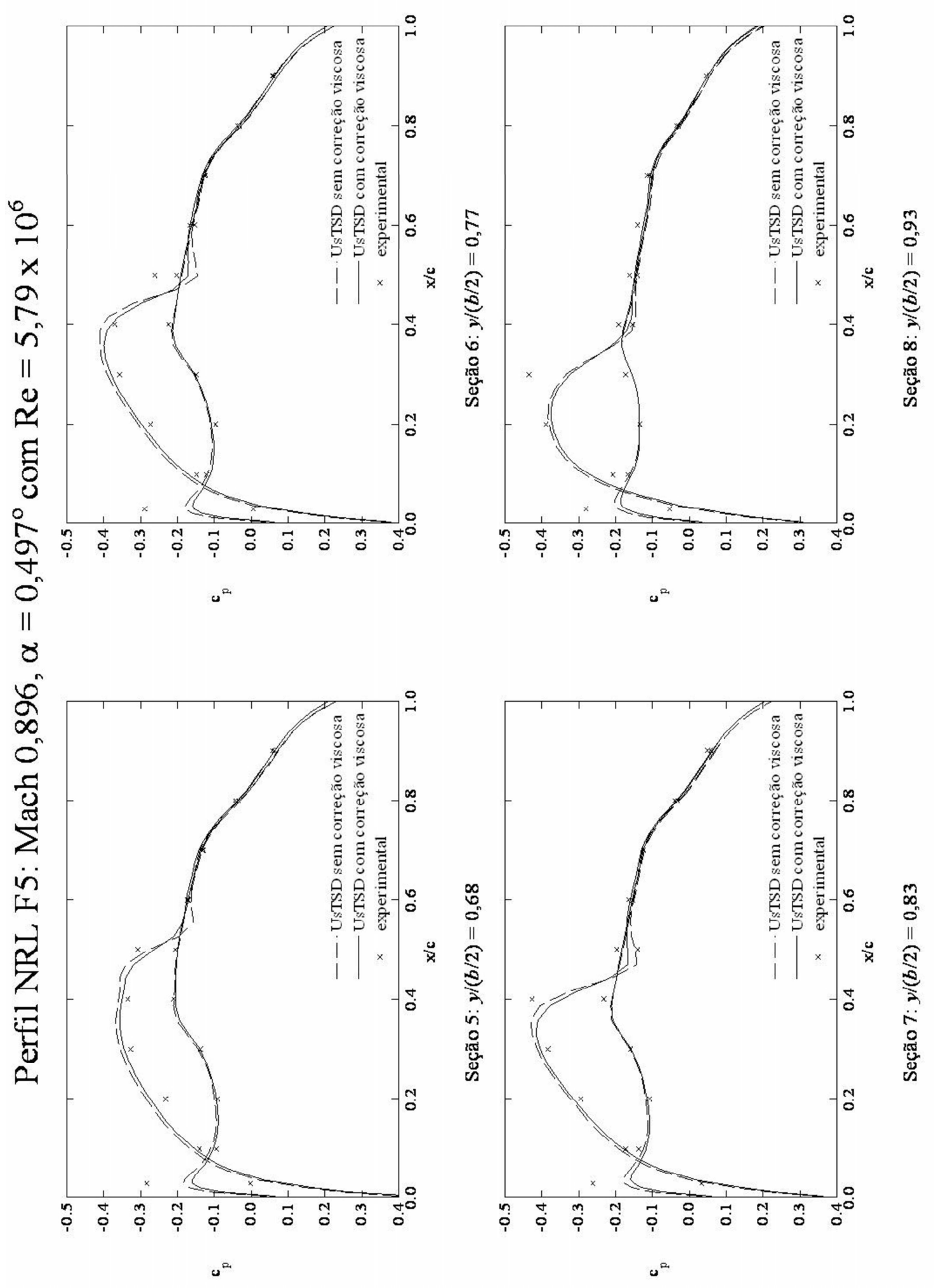

Figura 4.23: NRL F5 em Mach 0,896, $\alpha=0,497^{\circ} \operatorname{com} R e=5,79 \times 10^{6}$ (Parte II). 

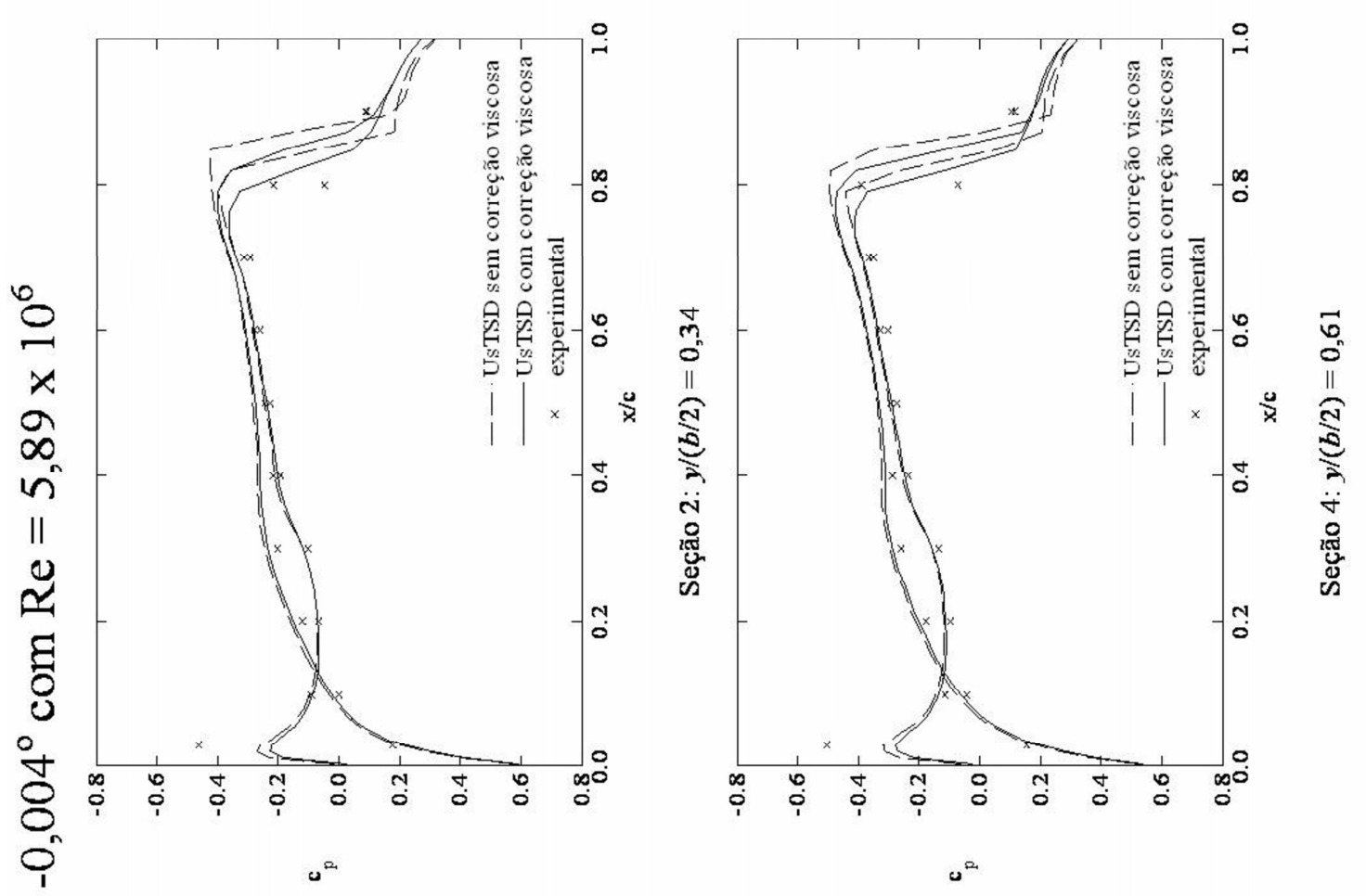

॥

$\gamma$
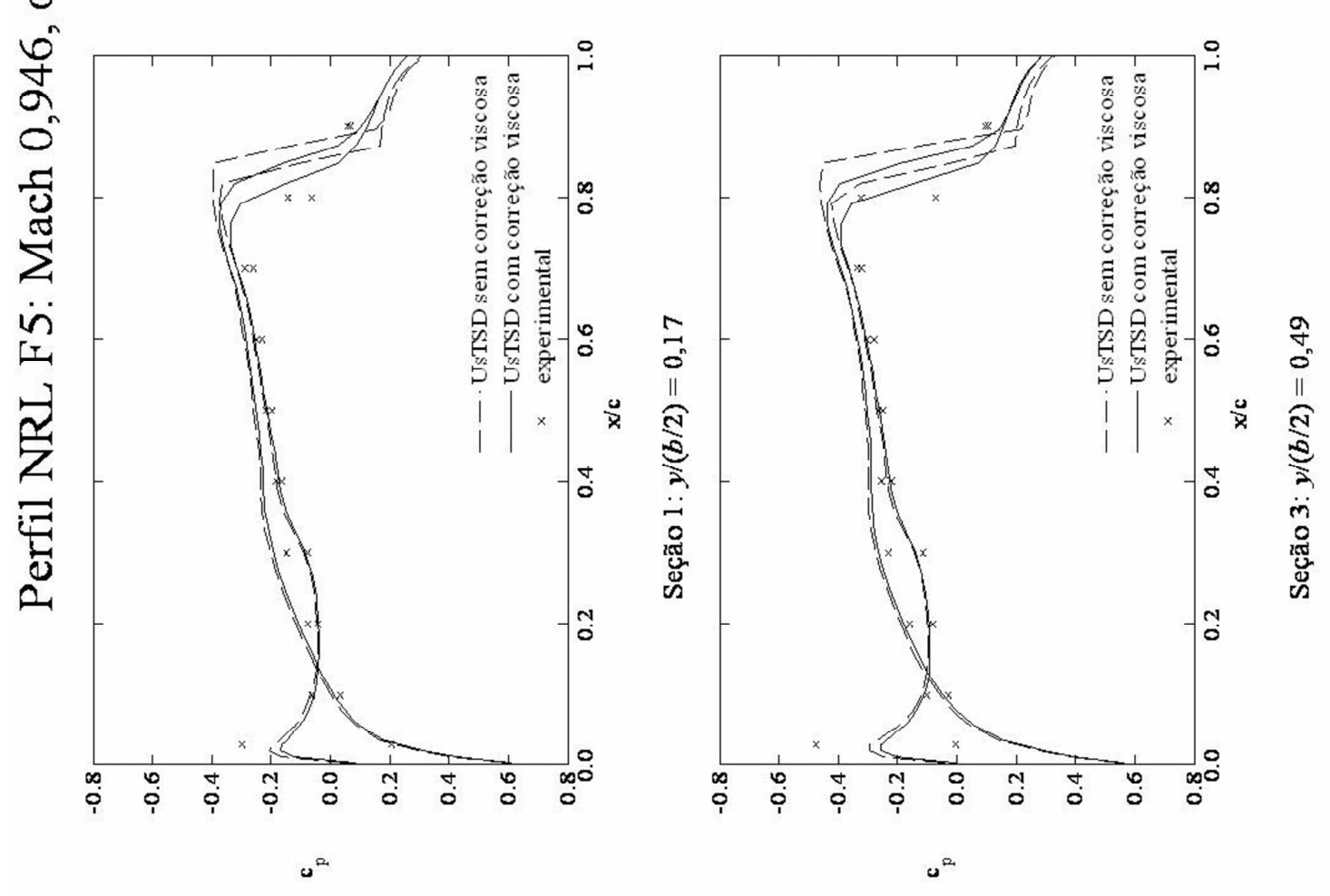

Figura 4.24: NRL F5 em Mach 0,946, $\alpha=-0,004^{\circ} \operatorname{com} R e=5,89 \times 10^{6}$ (Parte I). 

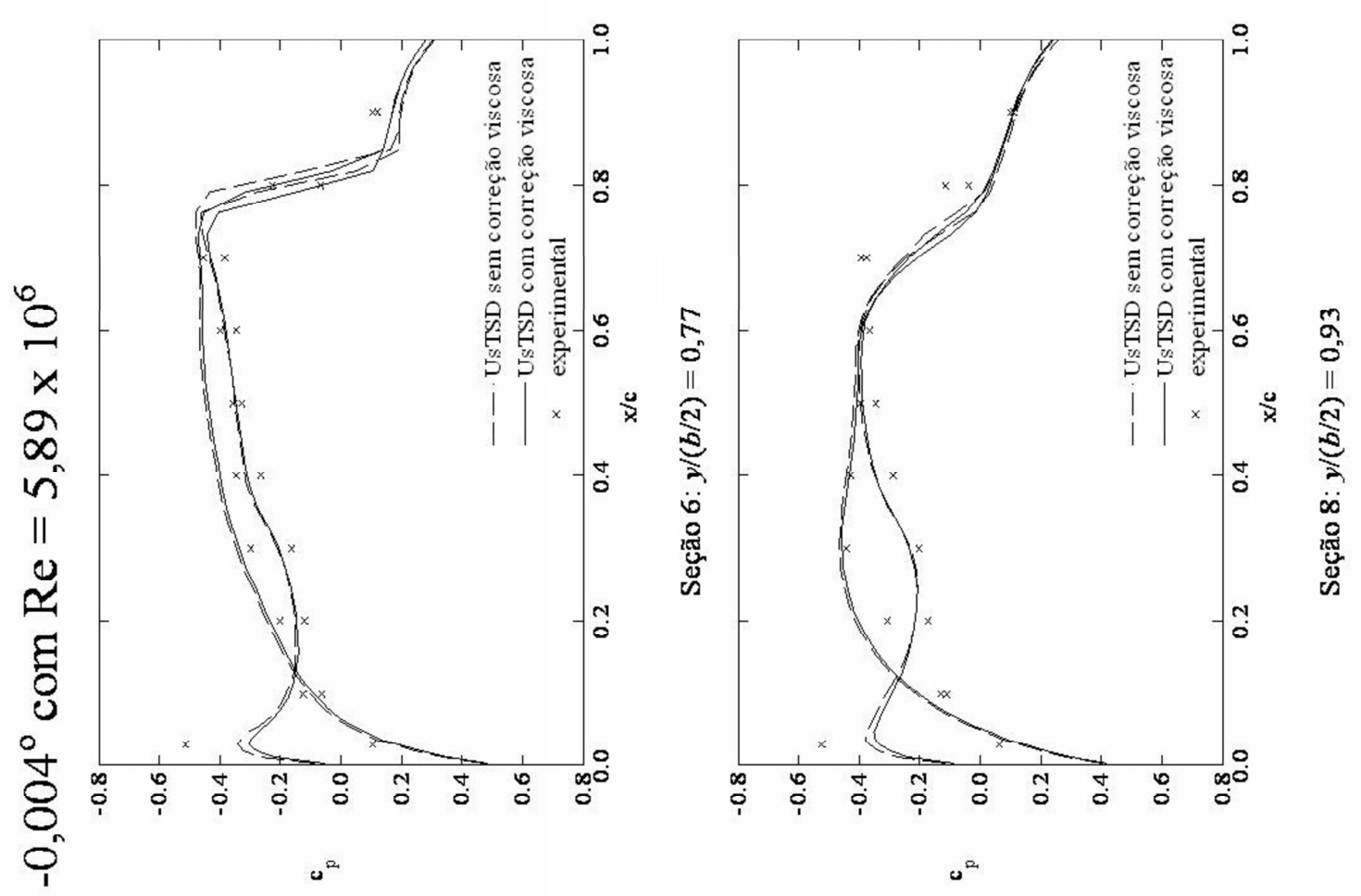

$\|$

$\gamma$
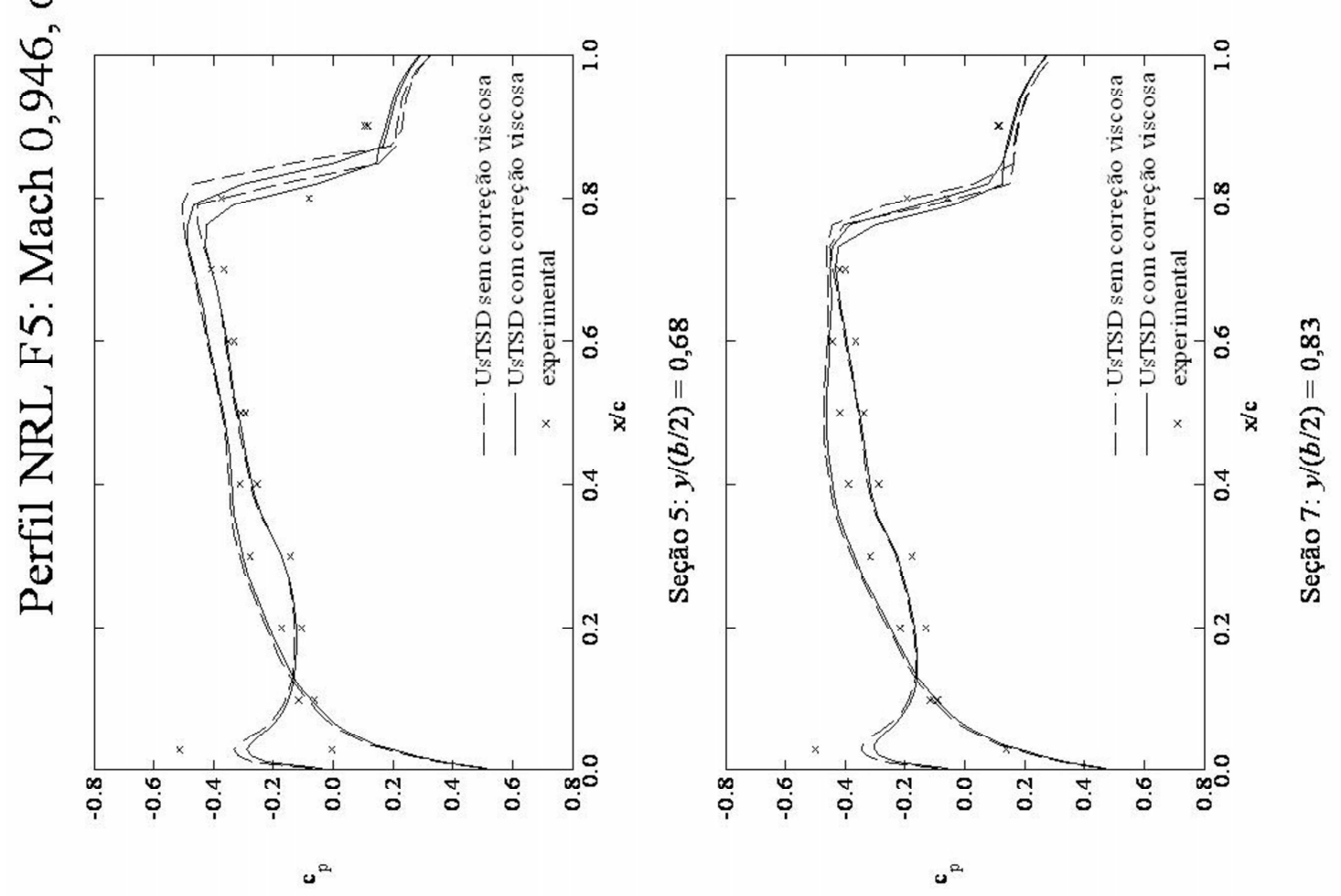

Figura 4.25: NRL F5 em Mach 0,946, $\alpha=-0,004^{\circ} \mathrm{com} R e=5,89 \times 10^{6}$ (Parte II). 


\subsection{Resultados do Programa UsTSD Não Estacionário}

Os resultados apresentados nesta sessão são simulações obtidas usando o programa UsTSD no caso não estacionário.

\subsubsection{Bidimensional}

\section{Seção de aerofólio NLR7301}

A primeira análise não estacionária foi para o perfil NLR7301. Foram utilizados dois conjuntos de resultados para esta seção de aerofólio:

- Fluxo estacionário com número de Mach igual a 0,752 e ângulo de ataque igual a $0,37^{\circ}$

- Fluxo não estacionário com número de Mach igual a 0,752, ângulo de ataque médio igual a $0,37^{\circ}$, oscilando em torno de $x / c=0,399$ com apmplitude de $2,01^{\circ}$ e frequência reduzida de 0,4 ,

ambos os casos com o número de Reynolds igual a 8,90 x $10^{6}$.

A geometria e os dados experimentais foram obtidos da Referência [22]. A Figura 4.26 mostra a distribuição de pressão para a seção NLR7301 $\operatorname{com} M_{\infty}=0,752, \alpha=0,37^{\circ}$, usando esquema conservativo, com e sem correção viscosa.

Apesar de aproximar os resultados numérico e experimental a correção viscosa não elimina diferenças importantes. A grande sucção no bordo de ataque destaca-se como uma das grandes diferenças em relação ao resultado experimental. Esta sucção ocorre porque o raio do bordo de ataque é bastante grande quando comparado com os outors perfis analisados. Fisicamente, no entanto, ela não ocorre. Lee $\left.{ }^{\lceil 2}\right\rceil$ sugere que se utilize a regra de Riegel para corrigir a inclinação do contorno da seção:

$$
f_{x m}=\frac{f_{x o}}{\sqrt{1+f_{x o}^{2}}}
$$

onde $f_{x o}$ e $f_{x m}$ são as inclinações do perfil original e modificado, respectivamente. O efeito dessa modificação ainda não foi investigado. Os resultados numérico e experimental estão mais próximos, especialmente para o caso não conservativo com correção viscosa. O pico 


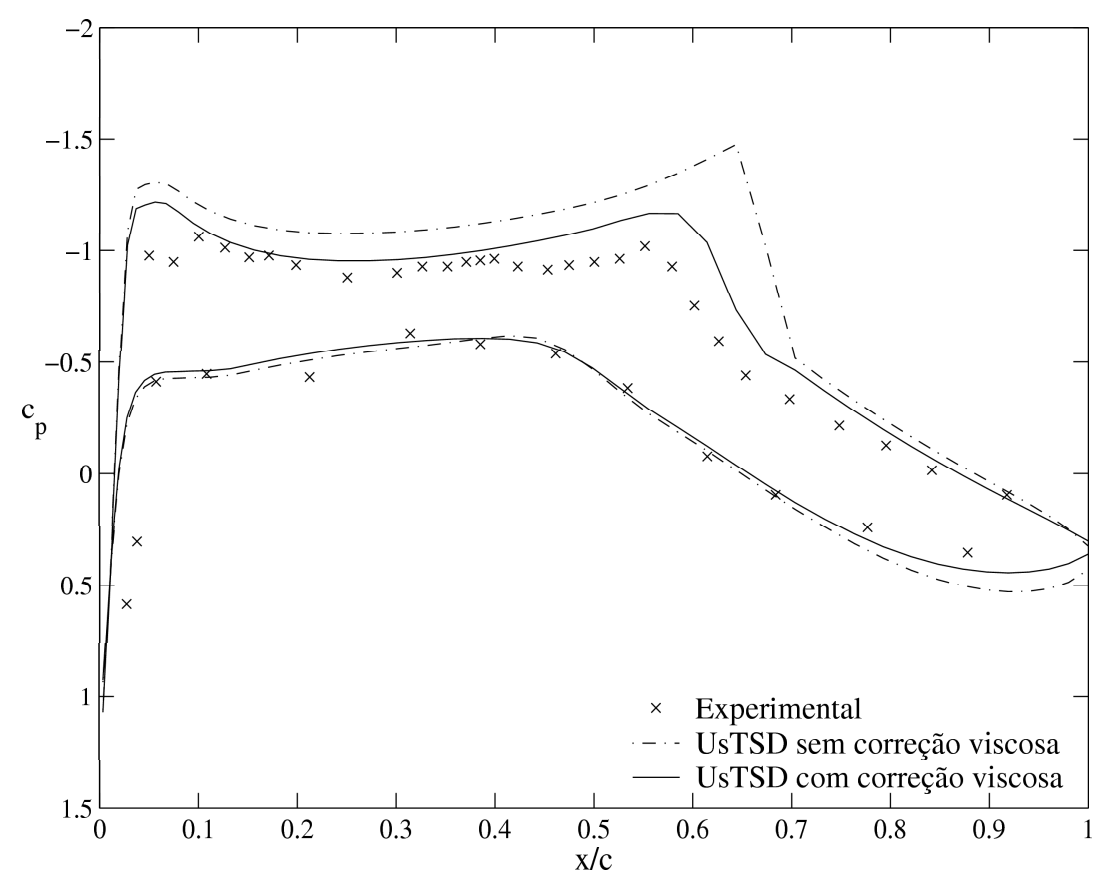

Figura 4.26: Coeficiente de pressão para a seção NLR7301 com $M_{\infty}=0,752, \alpha=0,37^{\circ}$, usando esquema conservativo, com e sem correção viscosa.

de sucção no bordo de ataque foi bastante reduzido, mesmo sem a modificação com a regra de Riegel.

Na Figura 4.27 mostra a espessura de deslocamento após a convergência da solução.

A Figura 4.28 mostra a seção NLR7301 sem correção e com a correção da espessura de deslocamento, após a convergência, com $M_{\infty}=0,752$ e $\alpha=0,37^{\circ}$.

Na Figura 4.29 mostra a variação entre o $c_{p}$ estacionário, $c_{p}$ estacionário em-fase e o $c_{p}$ estacionário fora-de-fase, para seção NLR7301 com a correção da espessura de deslocamento, após a convergência, com $M_{\infty}=0,752$ e $\alpha=0,37^{\circ}$. Aqui pode-se notar que o $c_{p}$ estacionário está entre o estacionário em-fase (com as maiores amplitudes de $c_{p}$ na região antes do choque) e o estacionário fora-de-fase (com as menores amplitudes de $c_{p}$ na região antes do choque), o mesmo comportamento é notado quando analisamos o Mach em torno do perfil, como pode-se ver na Figura 4.30.

A Figura 4.31 mostra a distribuição da magnitude da variação no coeficiente de pressão para a seção NLR7301 $\operatorname{com} M=0,752, \alpha=0,37^{\circ}$, oscilando em torno de $x / c=0,399$ com amplitude de $2,01^{\circ}$ e frequência reduzida de 0,4 , usando esquema não conservativo, com e sem correção viscosa. A Figura 4.32 mostra a distribuição do ângulo de fase da variação 


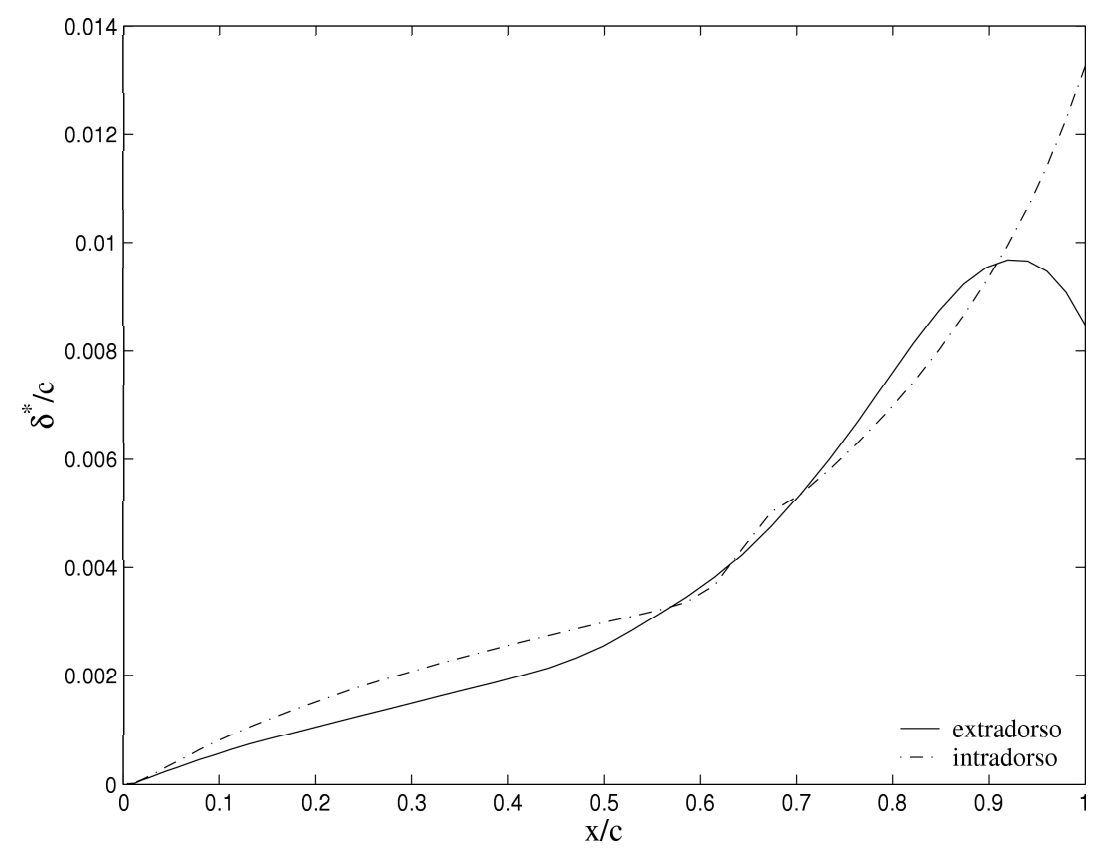

Figura 4.27: Espessura de deslocamento para a seção NLR7301 $\operatorname{com} M_{\infty}=0,752, \alpha=$ $0,37^{\circ}$, usando esquema conservativo.

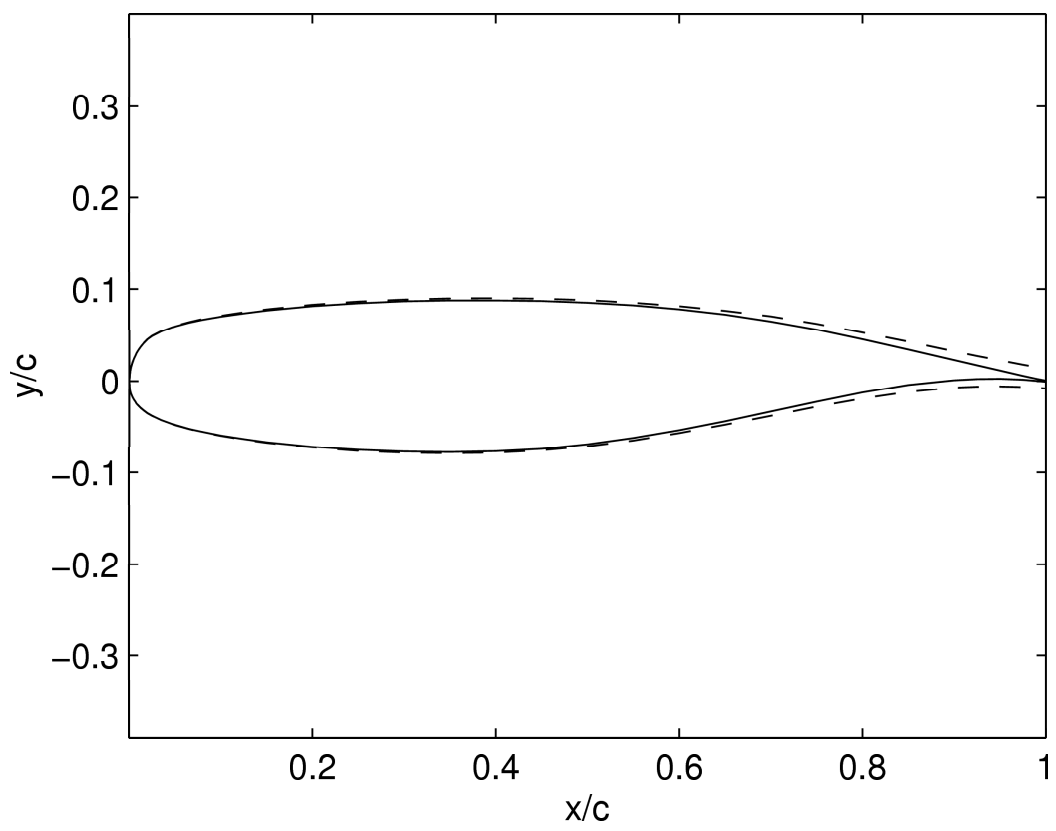

Figura 4.28: Correção da seção NLR7301, $\operatorname{com} M_{\infty}=0,752$ e $\alpha=0,37^{\circ}$, usando a espessura de deslocamento. 


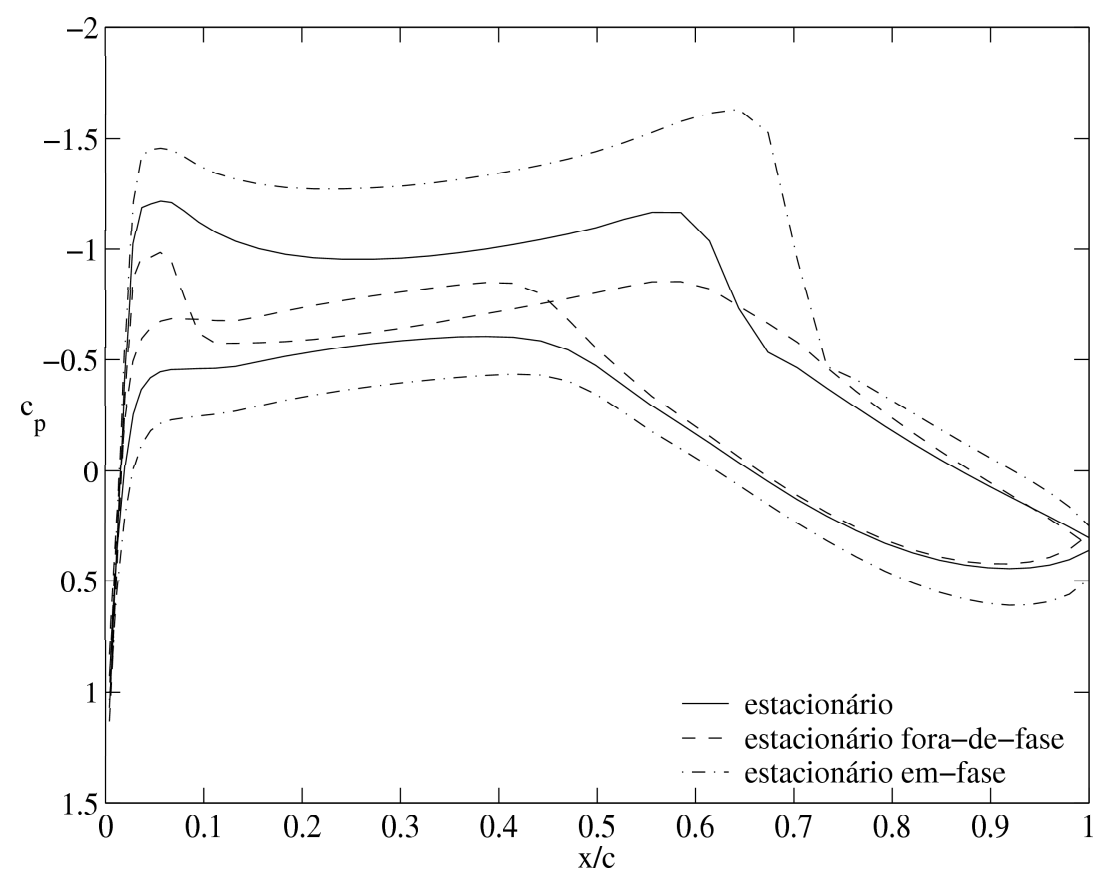

Figura 4.29: Coeficiente de pressão estacionário, estacionário em-fase e estacionário forade-fase para a seção NLR7301 $\operatorname{com} M_{\infty}=0,752, \alpha=0,37^{\circ}$, usando esquema conservativo, com correção viscosa.

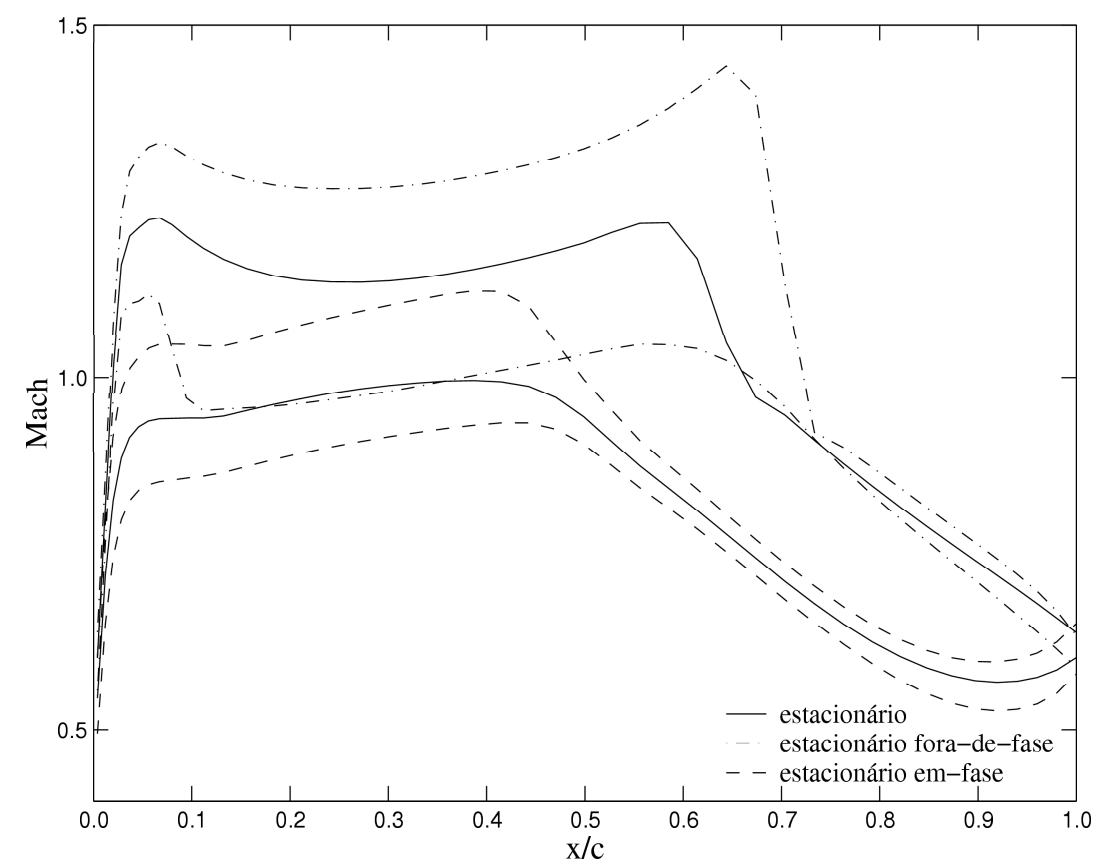

Figura 4.30: Distribuição de Mach no caso estacionário, estacionário em-fase e estacionário fora-de-fase na seção NLR7301 $\operatorname{com} M_{\infty}=0,752, \alpha=0,37^{\circ}$, usando esquema conservativo, com correção viscosa. 


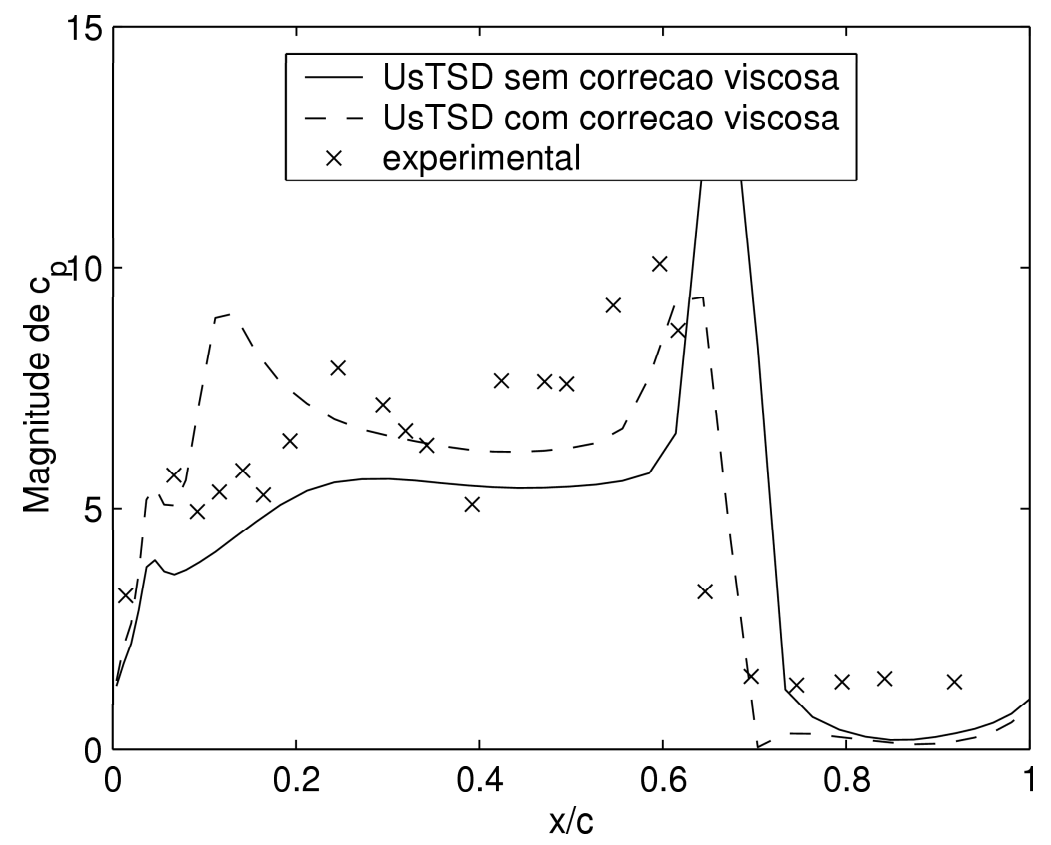

Figura 4.31: Distribuição da magnitude da variação no coeficiente de pressão para a seção NLR7301 com $M=0,752, \alpha=0,37^{\circ}$, oscilando em torno de $x / c=0,399$ com amplitude de $2,01^{\circ}$ e frequência reduzida de 0,4 , usando esquema não conservativo.

no coeficiente de pressão para a seção NLR7301 com $M=0,752, \alpha=0,37^{\circ}$, oscilando em torno de $x / c=0,399 \mathrm{com}$ amplitude de $2,01^{\circ}$ e frequência reduzida de 0,4 , usando esquema não conservativo, com e sem correção viscosa. O resultado não estacionário de magnitude também tem uma ligeira melhora com a correção viscosa. Não é possível tirar qualquer conclusão em relação ao ângulo de fase. A correção viscosa na análise não estacionária foi feita a partir da correção na solução não estacionária. Como tanto a solução em-fase quanto a solução fora-de-fase dependem da solução estacionária o efeito da correção é transmitido para a solução não estacionária. 


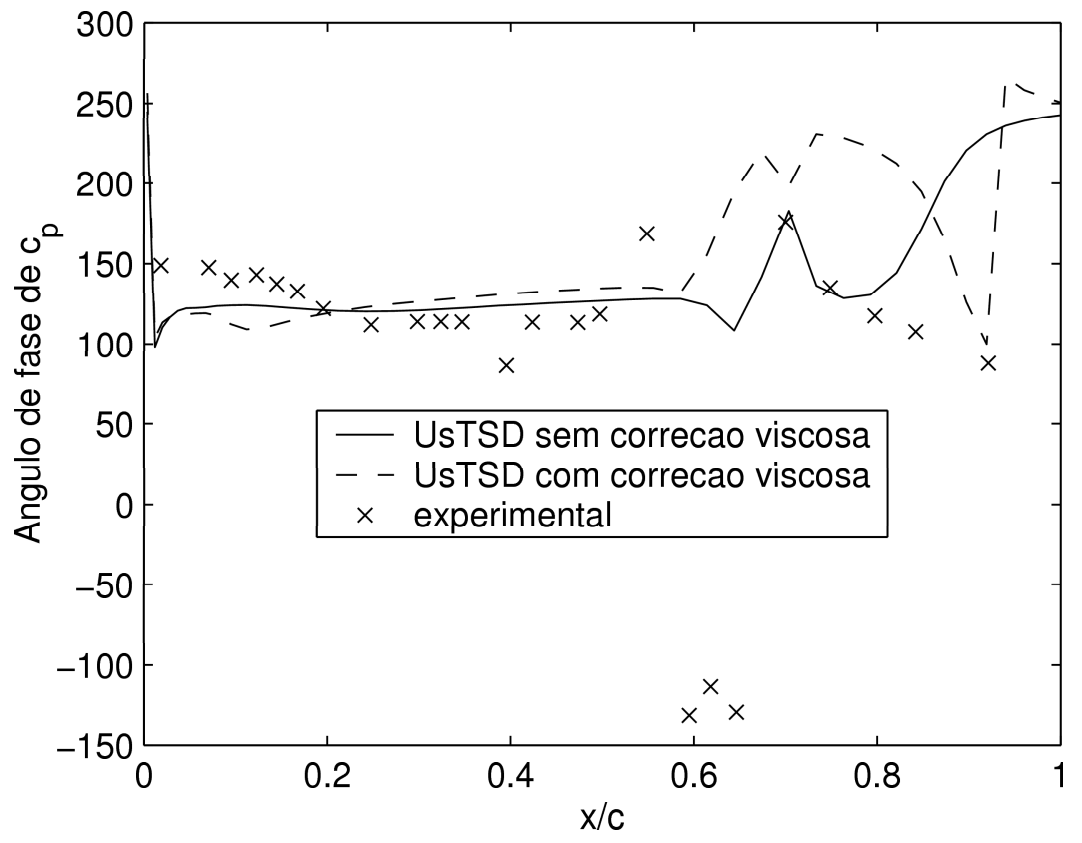

Figura 4.32: Distribuição do ângulo de fase da variação no coeficiente de pressão para a seção NLR7301 com $M=0,752, \alpha=0,37^{\circ}$, oscilando em torno de $x / c=0,399$ com amplitude de $2,01^{\circ}$ e frequência reduzida de 0,4 , usando esquema não conservativo.

\subsubsection{Tridimensional}

Da mesma maneira que as análies tridimensionais estacionárias, o perfil NRL F5 será usado para obter alguns resultados não estacionários e estas também serão comparadas a dados experimentais obtidos de Geurts ${ }^{[14]}$.

Os casos analisados são:

1. Mach 0,597, ângulo de ataque $=0,004^{\circ}, R e=4,57 \times 10^{6}$ e $\theta=0,115^{\circ}$;

2. Mach 0,896 , ângulo de ataque $=0,001^{\circ}, \operatorname{Re}=5,73 \times 10^{6}$ e $\theta=0,111^{\circ}$;

lembrando que $\theta$ é o ângulo de fase. Em cada um dos casos foram feitas três análises: estacionária, em-fase e fora de faze.

\section{Caso 1}

Note que os dados experimentais para o perfil NRL F5 em Mach 0,597, ângulo de ataque $=0,004^{\circ}, R e=4,57 \times 10^{6}$ e $\theta=0,115^{\circ}$ no caso estacionário não apresentaram a presença de ondas de choque como mostram as Figuras 4.33 e 4.34, isto é, o pefil se apresenta em 
condição subsônica. Como já visto na análise estacionária, os dados obtidos do código UsTSD com e sem correção viscosa apresentram uma boa correlação, principalmente nas seções centrais. Além disso, os dados do código UsTSD com e sem correção viscosa são praticamente idênticas, isto se deve ao fato da falta da presença do choque.

Ao analisar a parte em-fase, observa-se uma alta correlação ente os dados experimentais, o UsTSd sem correção viscosa e com correção viscosa, principalmente nas seções centrais como mostram as Figuras 4.35 e 4.36.

Já não se pode afirmar esta correlação entre os dados experimentais com os dados obtido do código UsTSD sem e com correção viscosa para o caso fora-de-fase, apesar que os resultados obtidos pelo código UsTSD apresentam características parecidas com os dados experimentais, como pode-se observar nas Figuras 4.37 e 4.38 .

\section{Caso 2}

Analisando o caso estacionário em que o perfil NRL F5 está em Mach 0,896, ângulo de ataque $=0,001^{\circ}, R e=5,73 \times 10^{6}$ e $\theta=0,111^{\circ}$, pode-se observar nas Figuras 4.39 e 4.40 que nos dados experimentais há a presença de ondas de choque fracas nas seções 7 e 8 (próximas a ponta da asa), porém o código UsTSD não detecta a presença destas ondas de choque e novamente as seções que obtiveram uma boa correlação com os dados do código UsTSD são as centrais $(4,5$ e 6$)$. A falta de detecção das ondas de choque tornou os dados obtidos do código UsTSD sem e com correção idênticas.

Porém ao analisar o caso em-fase, observou uma boa correlação entre as os dados do código UsTSD sem e com correção viscosa com os dados experimentais nas seções mais próximas a raiz da asa, e a medida que se afasta esta correlação piora como mostram nas Figuras 4.41 e 4.42

Já no caso fora-de-fase, há uma baixa correlação entre os dados obtidos do código UsTSD com os experimentais e a correlação piora mais a medida que se aproxima da ponta da asa, como mostram as Figuras 4.43 e 4.44. 

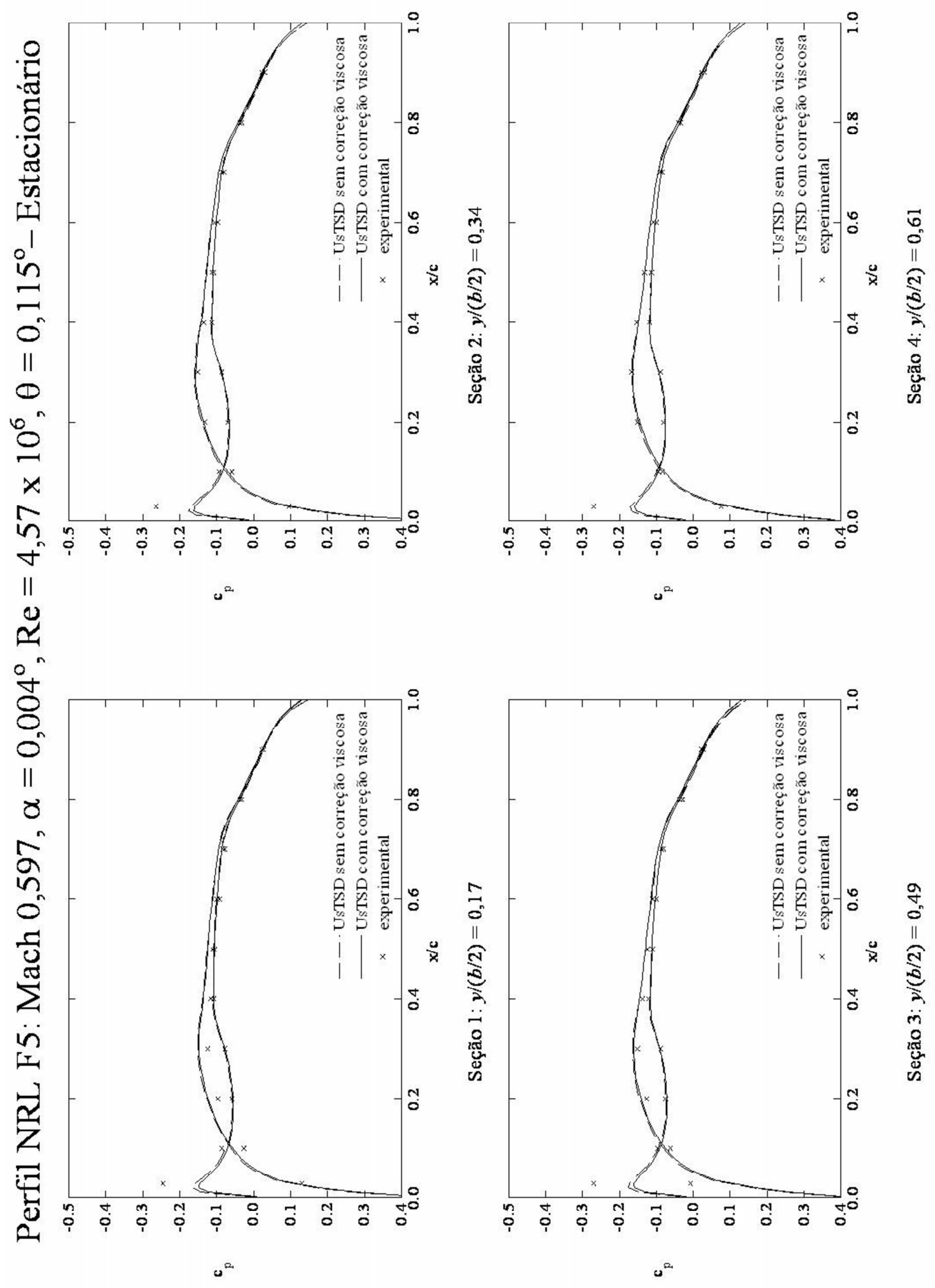

Figura 4.33: Análise da parte estacionária do perfil NRL F5 em Mach 0,597, ângulo de ataque $=0,004^{\circ}, R e=4,57 \times 10^{6}$ e $\theta=0,115^{\circ}$ (Parte I). 

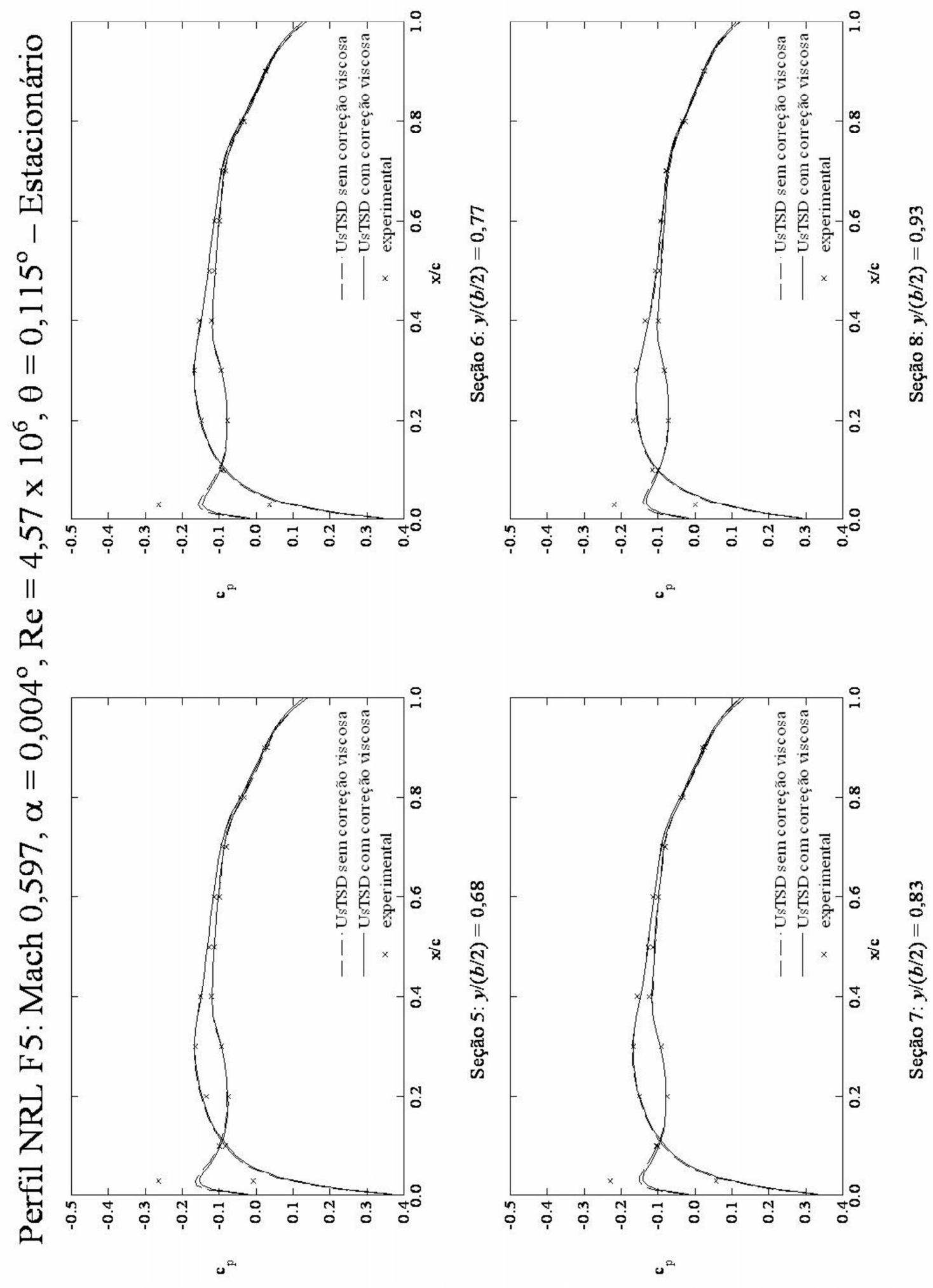

Figura 4.34: Análise da parte estacionária do perfil NRL F5 em Mach 0,597, ângulo de ataque $=0,004^{\circ}, \operatorname{Re}=4,57 \times 10^{6}$ e $\theta=0,115^{\circ}$ (Parte II). 

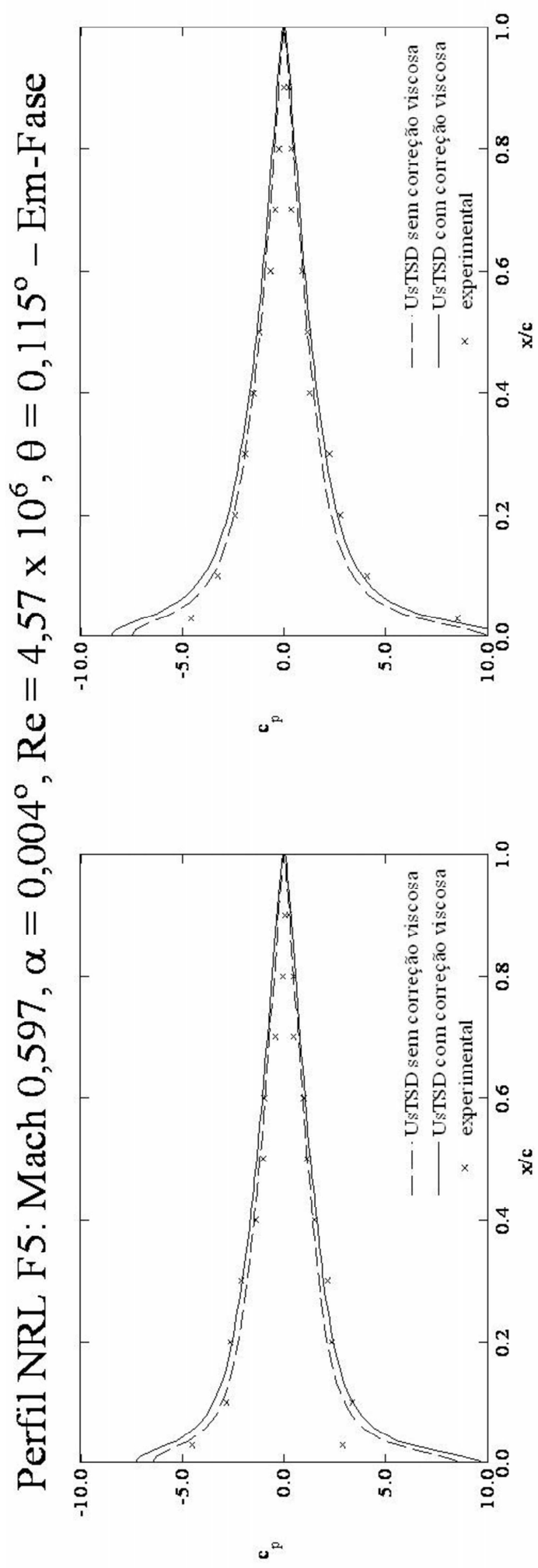
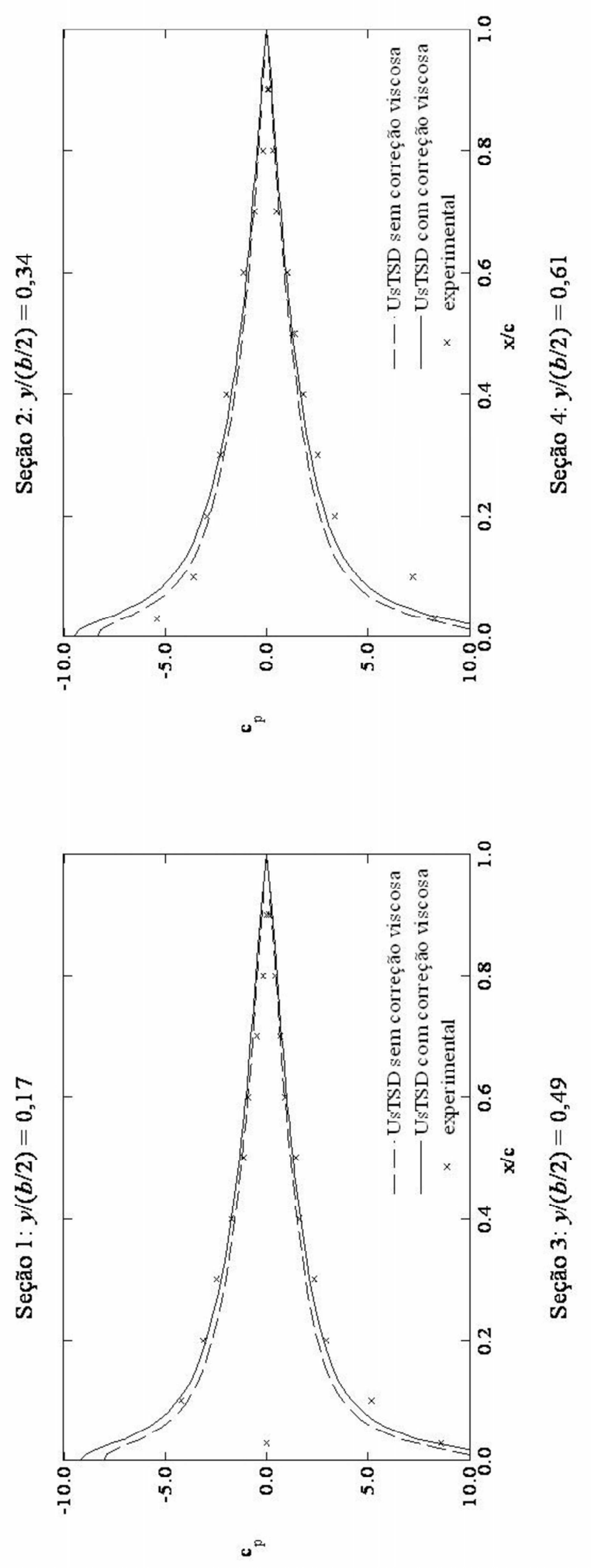

Figura 4.35: Análise da parte em-fase do perfil NRL F5 em Mach 0,597, ângulo de ataque = $0,004^{\circ}, R e=4,57 \times 10^{6}$ e $\theta=0,115^{\circ}$ (Parte I). 

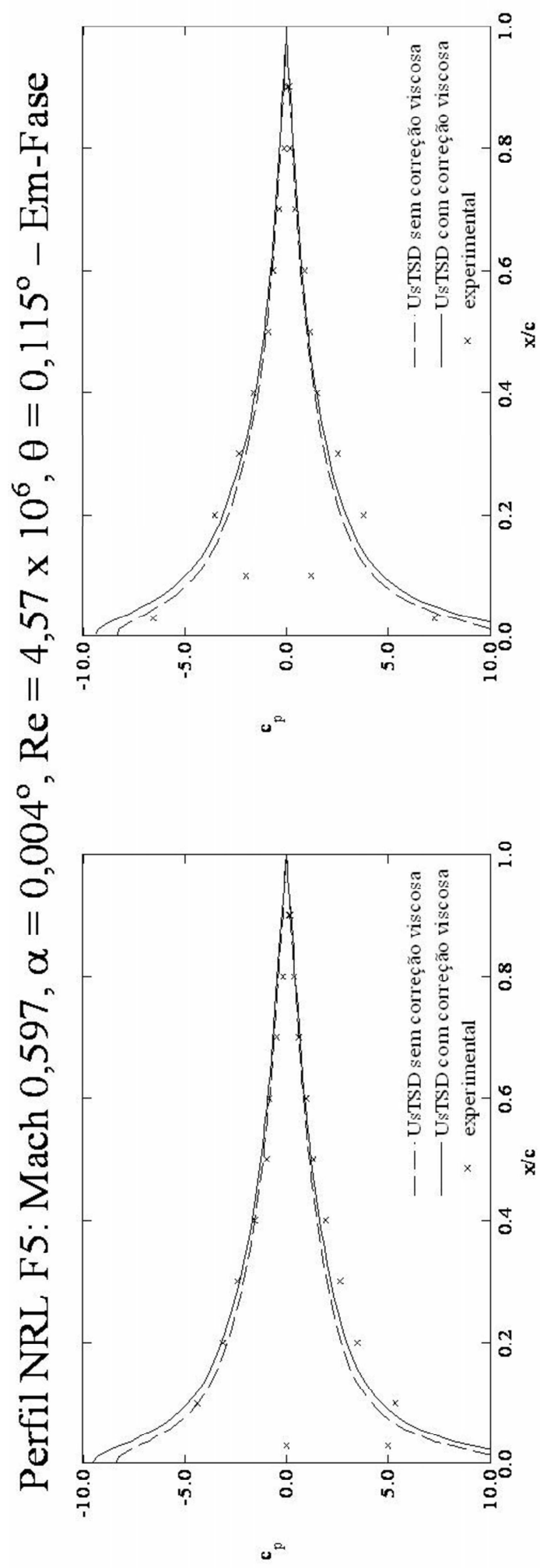
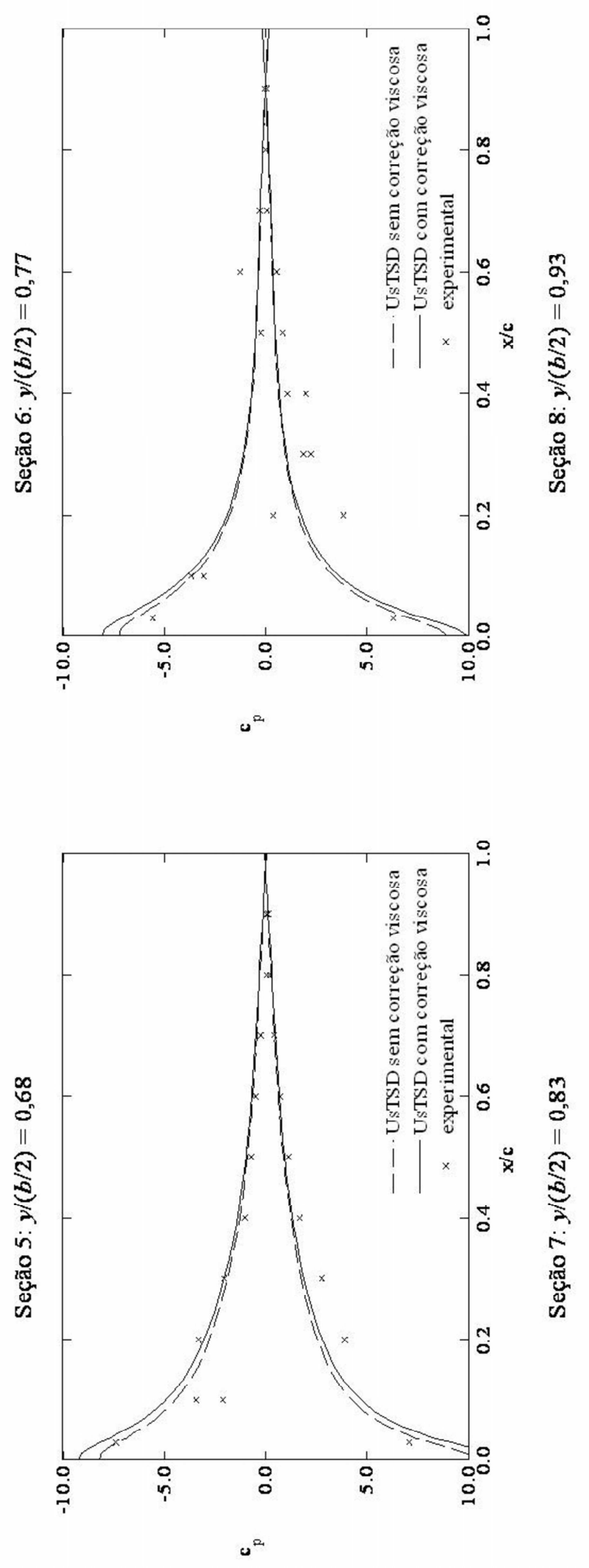

Figura 4.36: Análise da parte em-fase do perfil NRL F5 em Mach 0,597, ângulo de ataque = $0,004^{\circ}, R e=4,57 \times 10^{6}$ e $\theta=0,115^{\circ}$ (Parte II). 

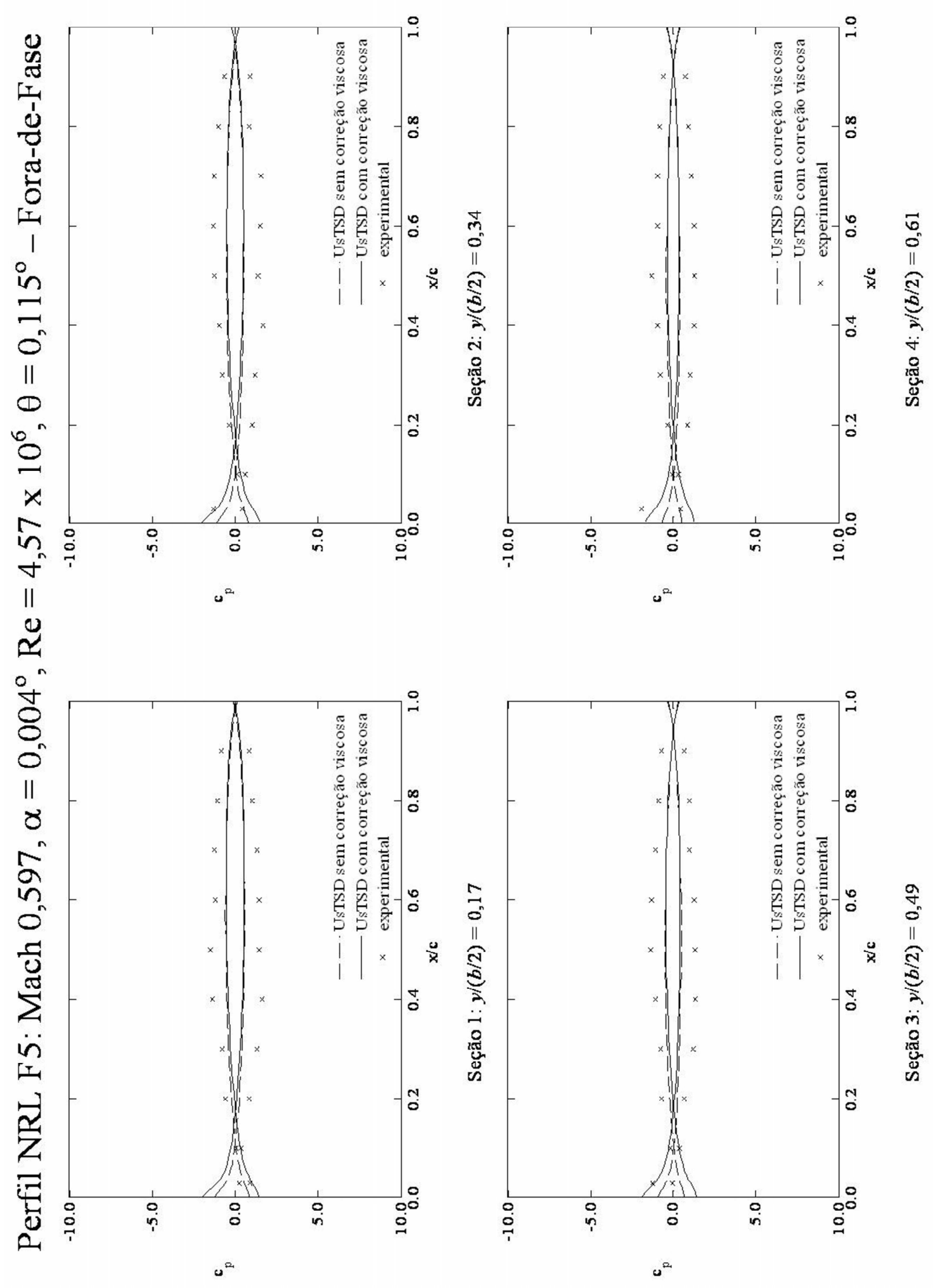

Figura 4.37: Análise da parte fora-de-fase do perfil NRL F5 em Mach 0,597, ângulo de ataque $=0,004^{\circ}, \operatorname{Re}=4,57 \times 10^{6}$ e $\theta=0,115^{\circ}$ (Parte I). 

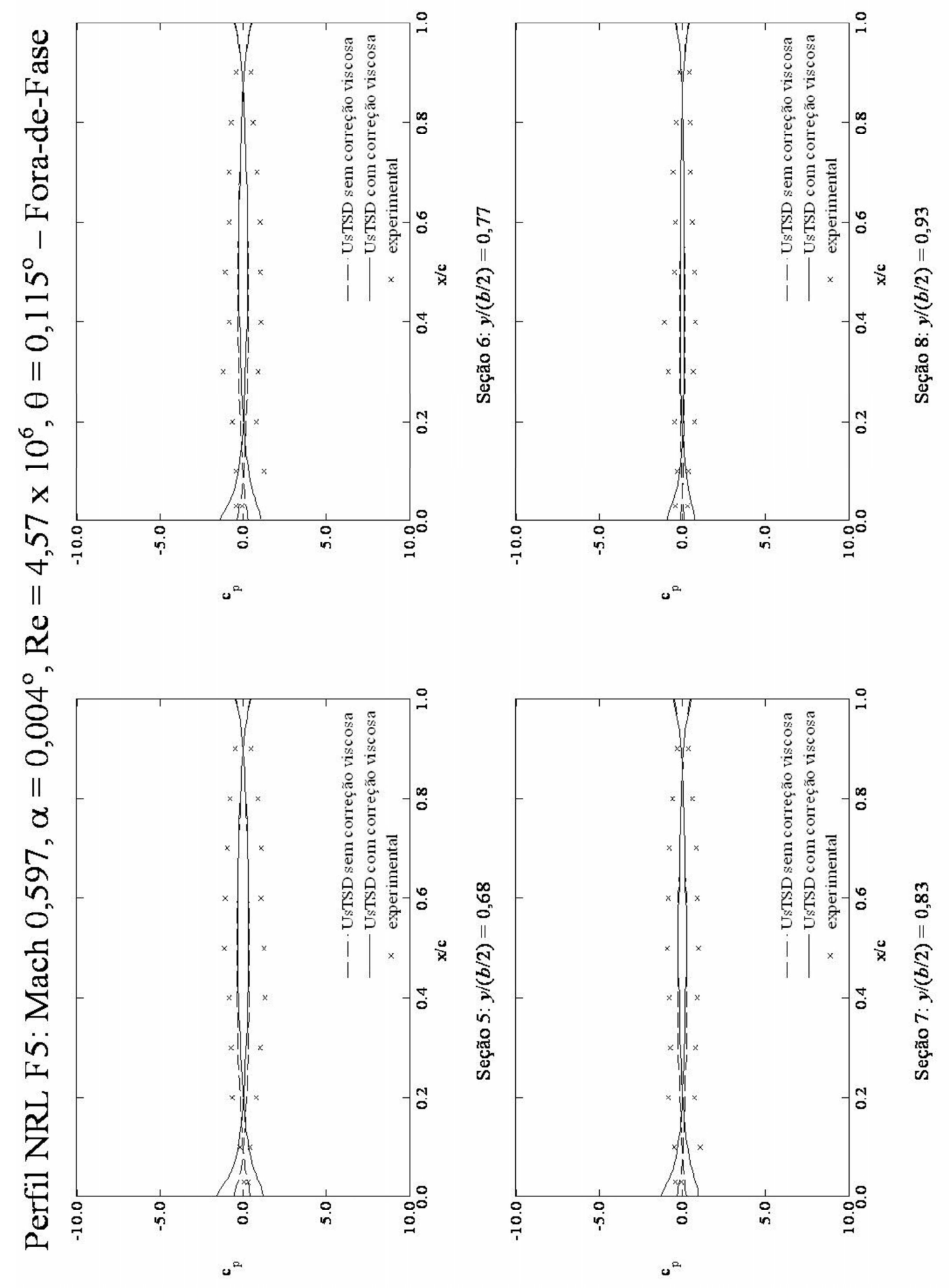

Figura 4.38: Análise da parte fora-de-fase do perfil NRL F5 em Mach 0,597, ângulo de ataque $=0,004^{\circ}, R e=4,57 \times 10^{6}$ e $\theta=0,115^{\circ}$ (Parte II). 

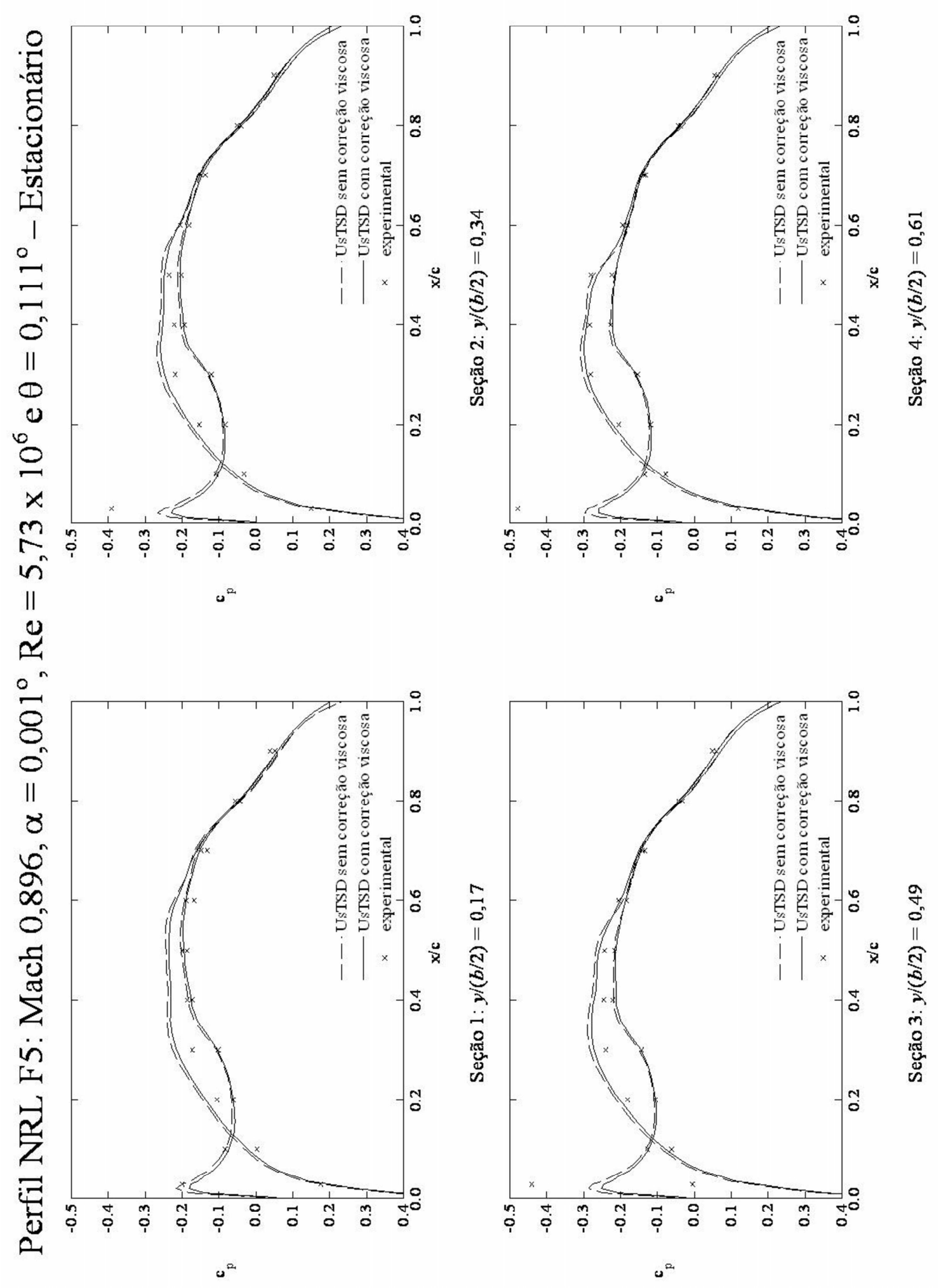

Figura 4.39: Análise da parte estacionária do perfil NRL F5 em Mach 0,896, ângulo de ataque $=0,001^{\circ}, \operatorname{Re}=5,73 \times 10^{6}$ e $\boldsymbol{\theta}=0,111^{\circ}($ Parte I $)$. 

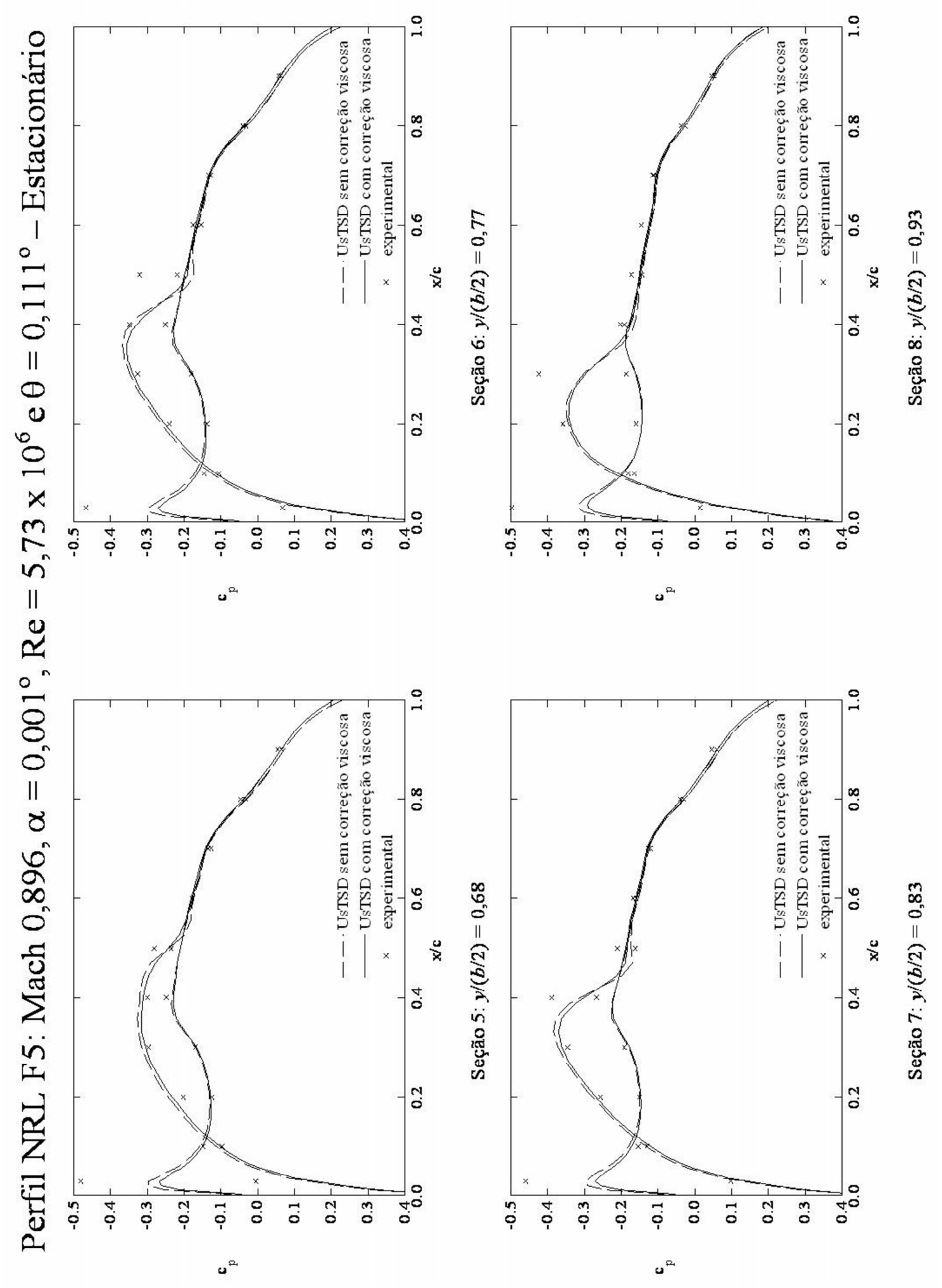

Figura 4.40: Análise da parte estacionária do perfil NRL F5 em Mach 0,896, ângulo de ataque $=0,001^{\circ}, R e=5,73 \times 10^{6}$ e $\theta=0,111^{\circ}$ (Parte II). 

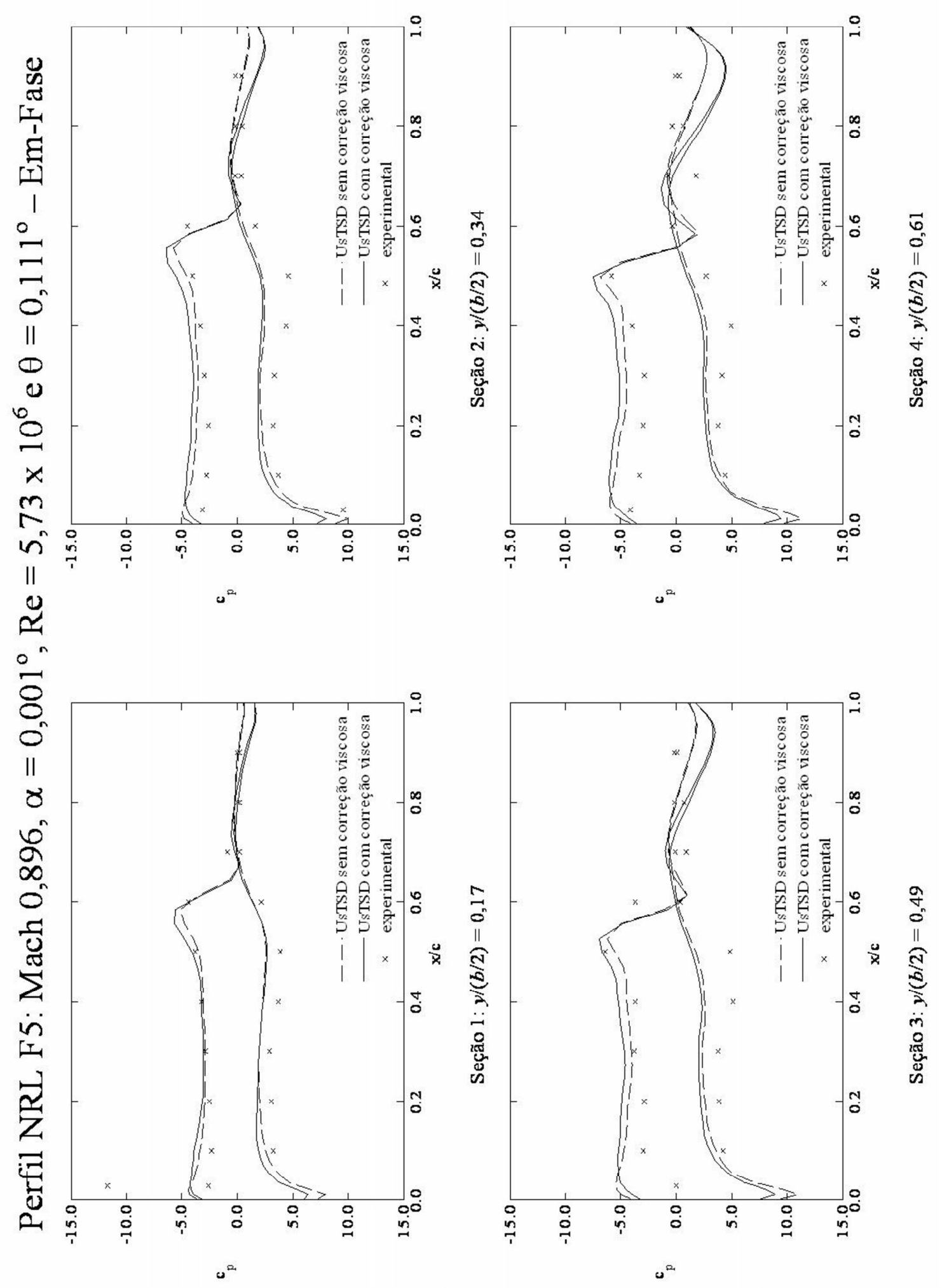

Figura 4.41: Análise da parte em-fase do perfil NRL F5 em Mach 0,896, ângulo de ataque = $0,001^{\circ}, R e=5,73 \times 10^{6}$ e $\theta=0,111^{\circ}$ (Parte I). 

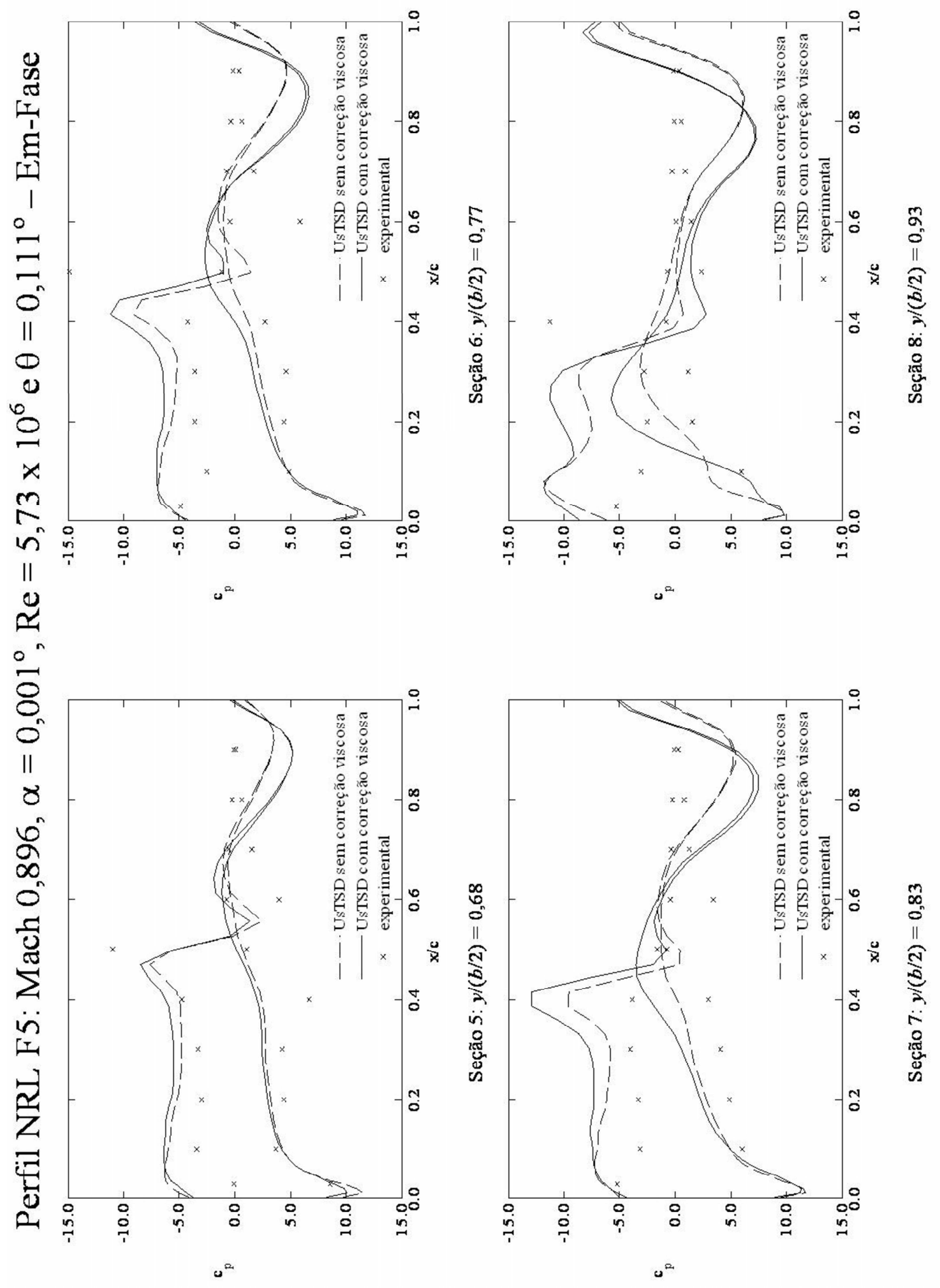

Figura 4.42: Análise da parte em-fase do perfil NRL F5 em Mach 0,896, ângulo de ataque = $0,001^{\circ}, R e=5,73 \times 10^{6}$ e $\theta=0,111^{\circ}$ (Parte II). 

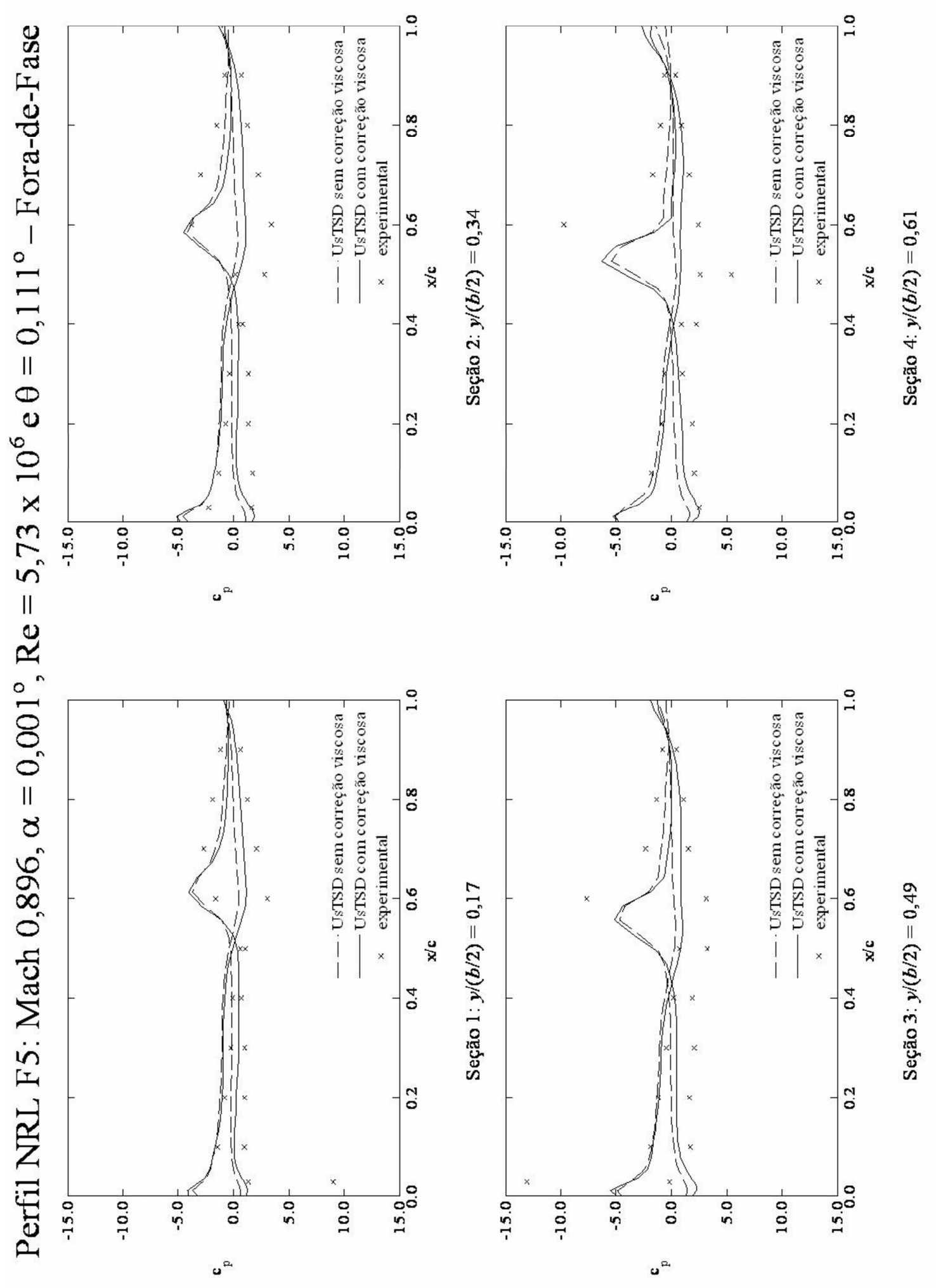

Figura 4.43: Análise da parte fora-de-fase do perfil NRL F5 em Mach 0,896, ângulo de ataque $=0,001^{\circ}, \operatorname{Re}=5,73 \times 10^{6}$ e $\theta=0,111^{\circ}$ (Parte I). 

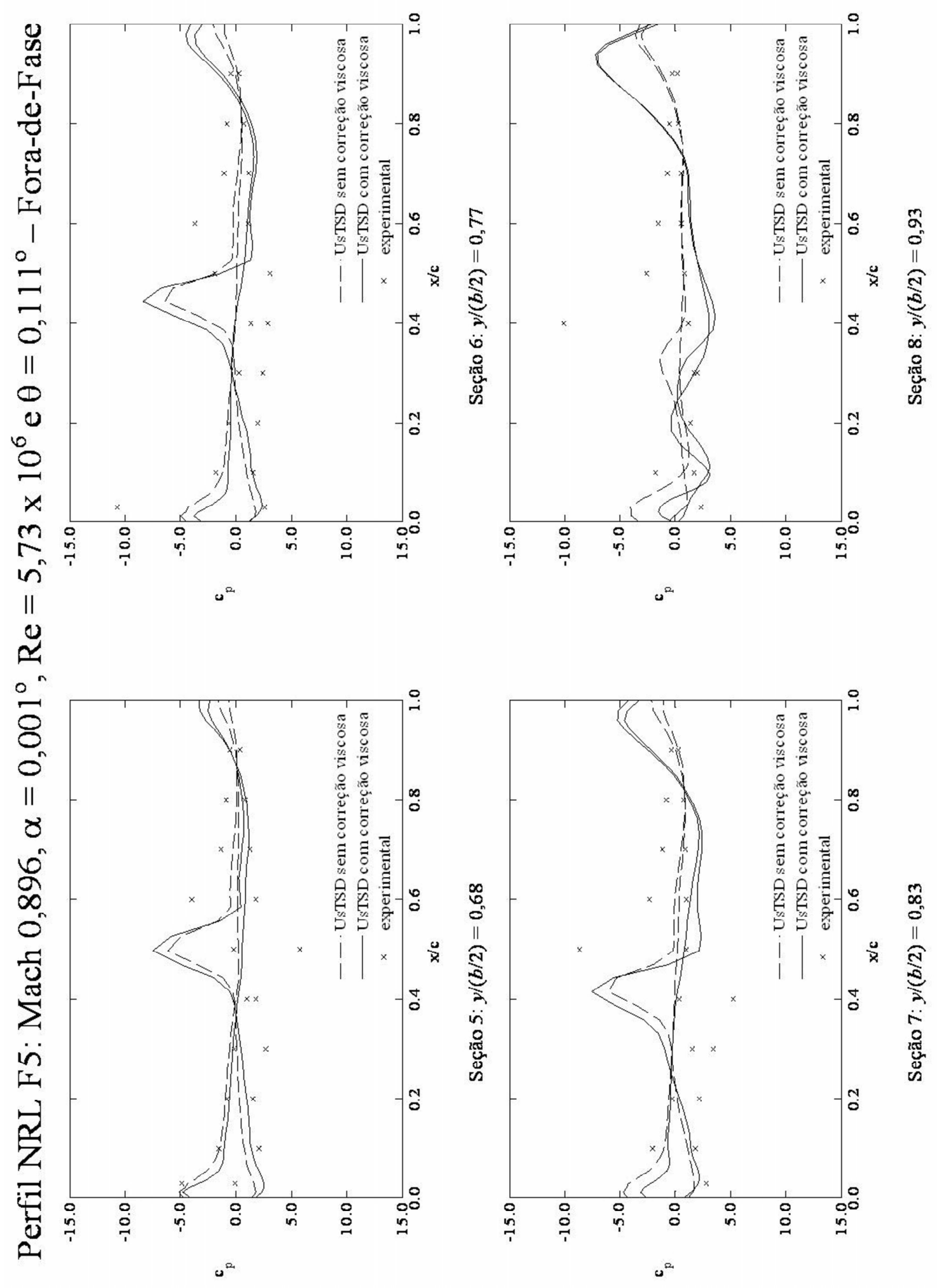

Figura 4.44: Análise da parte fora-de-fase do perfil NRL F5 em Mach 0,896, ângulo de ataque $=0,001^{\circ}, \operatorname{Re}=5,73 \times 10^{6}$ e $\theta=0,111^{\circ}$ (Parte II). 


\section{Capítulo 5}

\section{Conclusões e Trabalhos Futuros}

\subsection{Conclusão}

A análise no domínio da freqüência apresenta vantagens significantes à analise no domínio do tempo. Na análise no domínio do tempo, o tamanho do passo tempo e o tempo total exigido para a análise pode impor um custo computacional muito maior do que a análise no domínio da freqüência ${ }^{[}$. , além disso há algumas técnicas que permitem transformações para o domínio da freqüência mantendo a não linearidade do problema. A técnica usada neste estudo é a "média harmônica" [27], que mantém as condições não lineares dos termos no espaço mas assumindo uma variação harmônica nas variáveis dependentes do tempo.

Além disso, os dados obtidos das análises não-estacionárias no domínio do tempo são transformados no domínio da freqüência para poder observar e analisar um período da oscilação, com isso pode comprometer algumas características não lineares do problema.

O objetivo principal da pesquisa foi o estudo de métodos para simulação da interação entre choque e camada limite, na solução do escoamento potencial de pequenas perturbações em regime transônico no domínio da freqüência. Este objetivo foi alcançado quando se considera que os métodos de correção já foram implementados com sucesso no caso estacionário.

Já a implementação no caso não estacionário em partes obteve-se sucesso, considerando que os resultados próximo a raiz da asa, a parte em-fase mostram boa correlação e as forade-fase apresentaram o comportamento dos dados experimentais.

Ao aplicar a correção viscosa nas equações de pequenas perturbações o efeito de deslo- 
camento da posição da onda de choque a montante e a redução da sua intensidade foi obtido, conforme os programas TSD+Ent+Vort, TSD+Ent+Vort+CL e UsTSD com correção viscosa mostraram nos Capítulos 3 e 4.

Como observado, o método de correção viscosa apresentou melhores resultados, compensando-se um possível aumento do custo computacional em torno de $10 \%$ no tempo. O menor número de iterações, dependendo do caso, pode até diminuir o tempo computacional como mostra no Capítulo 4. Além disso, a fácil implementação torna esta correção atraente.

Os resultados apresentados no Capítulo 3, mostraram que as equações de pequenas perturbações transônica com correção de entropia e vorticidade sem (TSD+Ent+Vort) e com correção de viscosidade (TSD+Ent+Vort+CL) apresentam bons resultados comparados aos dados simulados vistos nas referências Lee ${ }^{[22]}$ e Batina ${ }^{[5]}$.

Além disso, no Capítulo 4, observou-se que a Teoria de Pequenas Perturbações apresenta bons resultados e que os códigos desenvolvidos durante o doutorado, TSD+Ent+Vort e TSD+Ent+Vort+CL, são consistentes e apresentam resultados coerentes quando comparados ao UsTSD.

No Capítulo 4 pode-se observar também que o método de correção viscosa no caso não estacionário apresentou melhores resultados, além disso, a fácil implementação torna esta correção atraente e esta será implementada em análises tridimensionais.

\subsection{Sugestões para Trabalhos Futuros}

O presente estudo revelou que a Teoria de Pequenas Perturbações analisada no domínio da frequência com correção viscosa apresenta uma série de vantagens, como a fácil implementação e principalmente o baixo custo computacional. A implementação da correção viscosa obteve-se sucesso quando considerado o fluxo estacionário, quanto ao fluxo nãoestacionário, apesar de apresentar bons resultados nas seções intermediárias (afastando-se um pouco da raiz e da ponta de asa), há necessidade de mais investigações, com isto, segue algumas considerações e sugestões para trabalhos futuros:

- Como foi visto no Capítulo 4, há uma necessidade em aprofundar a análise do programa UsTSD, isto é, buscar mais resultados experimentais não estacionários e comparar com os dados gerados pelo código UsTSD. Além disso, há uma necessidade 
em investigar as causas da baixa correlação entre os dados do código UsTSD obtidos próximo a raiz e próximos a ponta da asa com os dados experimentais.

- Comparar o código UsTSD com outros códigos existentes (exemplo: CFX), verificando a performance e desempenho da obtenção dos resutados.

- Utilizar outros métodos de correção viscosa para comparação, com o intuito de verificar a eficácia deste método.

- Usar a mesma correção viscosa no código Euler. 


\section{Apêndice A}

\section{Resultados Bidimensionais Subsônicos}

Os resultados apresentados neste capítulo são simulações usando as equações de pequenas perturbações transônico com correção de entropia e vorticidade, sem $(T S D+E n t+V o r t)$ e com correção viscosa $(T S D+E n t+V o r t+C L)$, desenvolvidos e baseados nas descrições da seção 2.5. Todos os casos apresentados neste apêndice são analisados e comparados com resultados da literatura ${ }^{[1]}$. O objetivo das simulações é testar os diferentes tipos de perfis aplicados a números de Mach subsônicos.

\section{A.1 Perfis}

Os três perfis a serem tratados neste capítulo são NACA0012, NACA0006 e NACA0015, e as suas geometrias são mostradas nas Figuras A.1, A.2 e A.3, repesctivamente. Os dados geométicos destes perfis foram obtidos do UIUC Airfoil Coordinates Datahase ${ }^{[36]}$ e serão testados a números de Mach de entrada $\left(M_{\infty}\right)$ igual a 0,001 a qual pode-se considerar esta como 0 (zero), e ângulo de ataque $(\alpha)$ igual a $0^{\circ}$.

Figura A.1: Seção do aerofólio NACA0012. 
Figura A.2: Seção do aerofólio NACA0006.

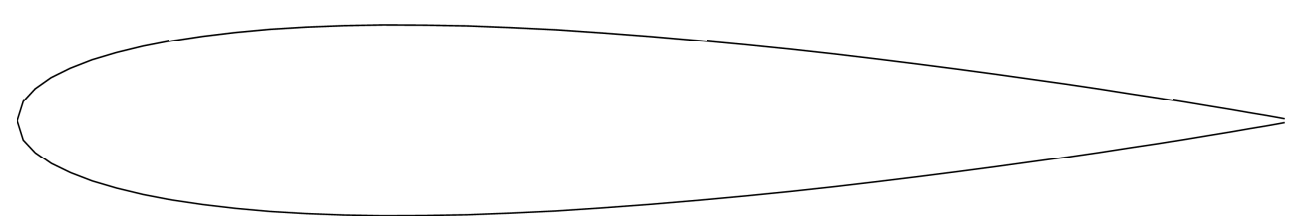

Figura A.3: Seção do aerofólio NACA0015.

\section{A.2 Critério de Convergência}

A convergência para estes casos foram ou ter residual ser menor que $10^{-8}$, ou ter residual relativo, razão entre o residual máximo e o residual médio, ser menor que 1,01. As Figuras A.4, A.5 e A.6 mostram que as convergências são uniformes e sem grandes variações para os perfis NACA0012, NACA0006 e NACA0015, respectivamente.

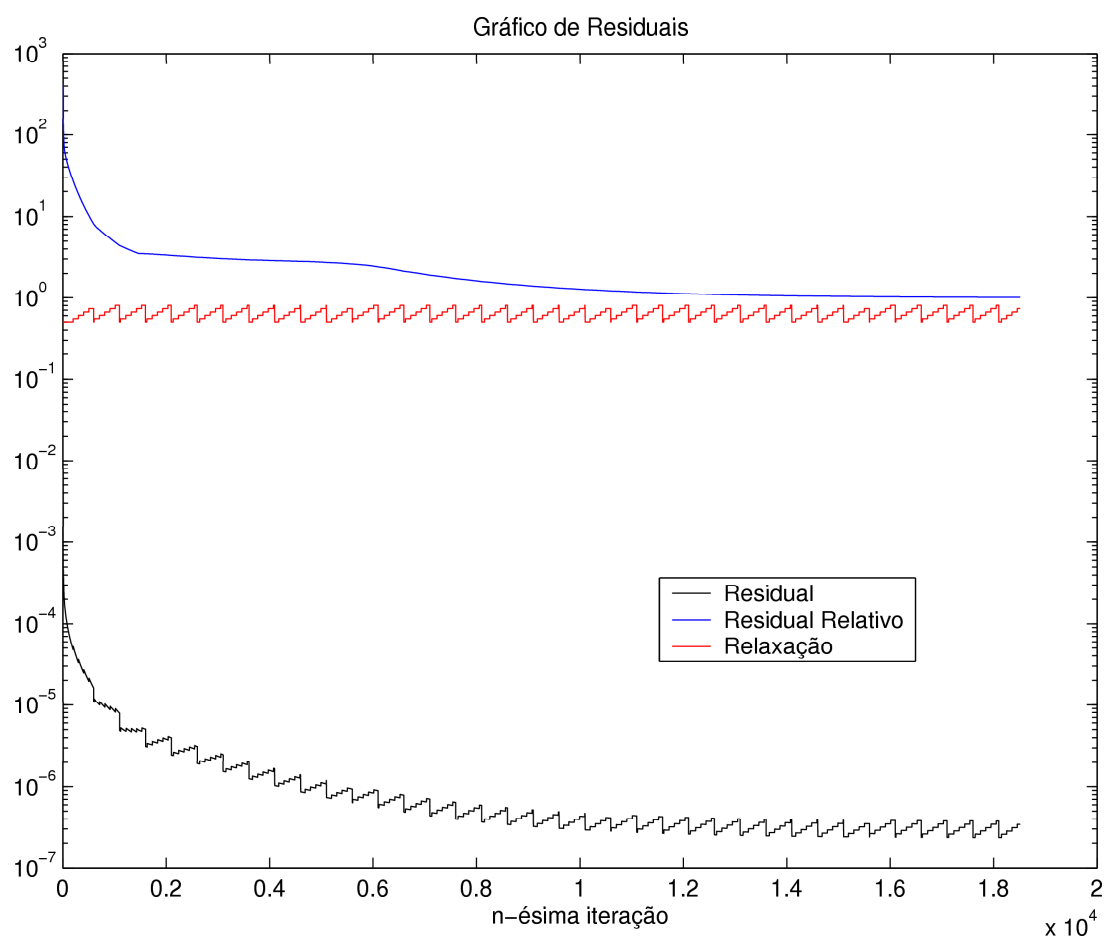

Figura A.4: Gráfico do residual usando TSD+Ent+Vort+CL para seção do perfil $\mathrm{NACA} 0012 \operatorname{com} M_{\infty}=0$ e $\alpha=0,00^{\circ}$. 


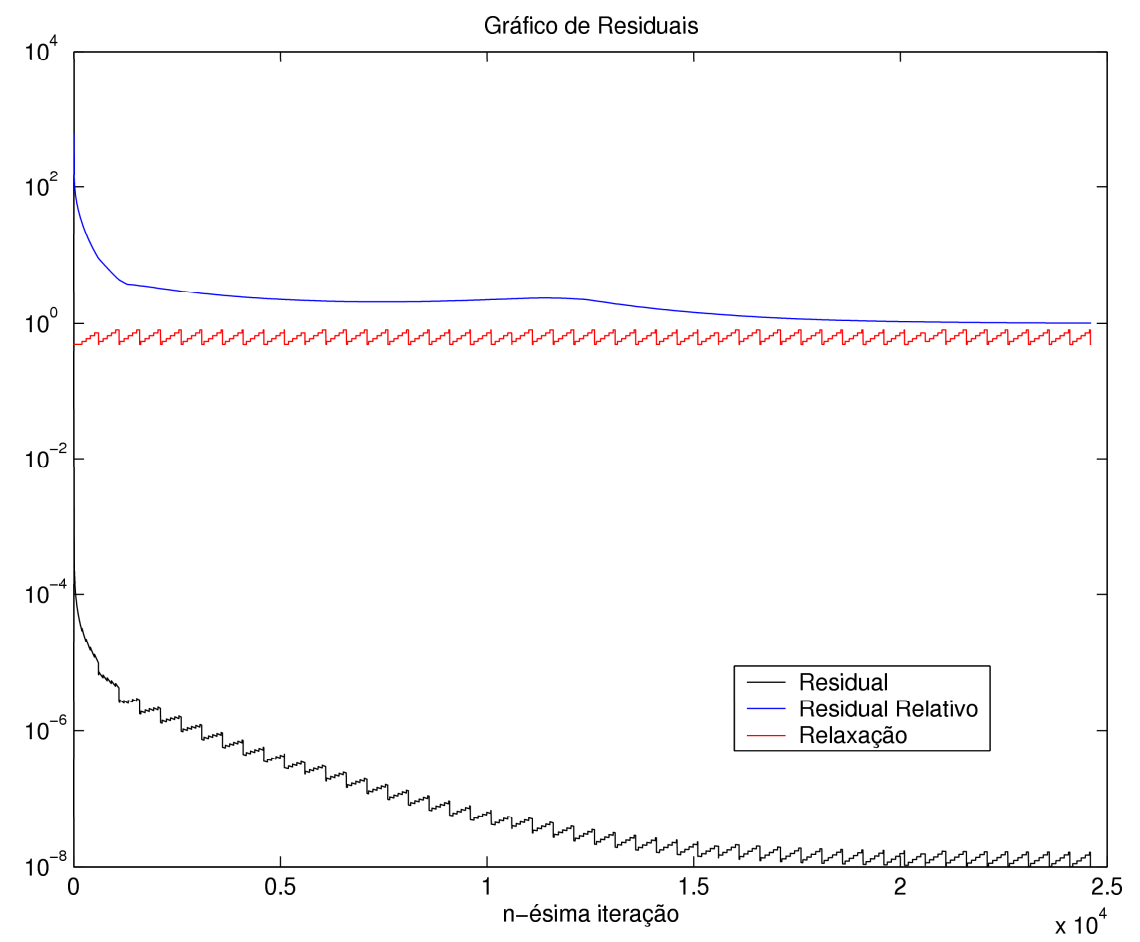

Figura A.5: Gráfico do residual usando TSD+Ent+Vort+CL para seção do perfil NACA0006 $\operatorname{com} M_{\infty}=0$ e $\alpha=0,00^{\circ}$.

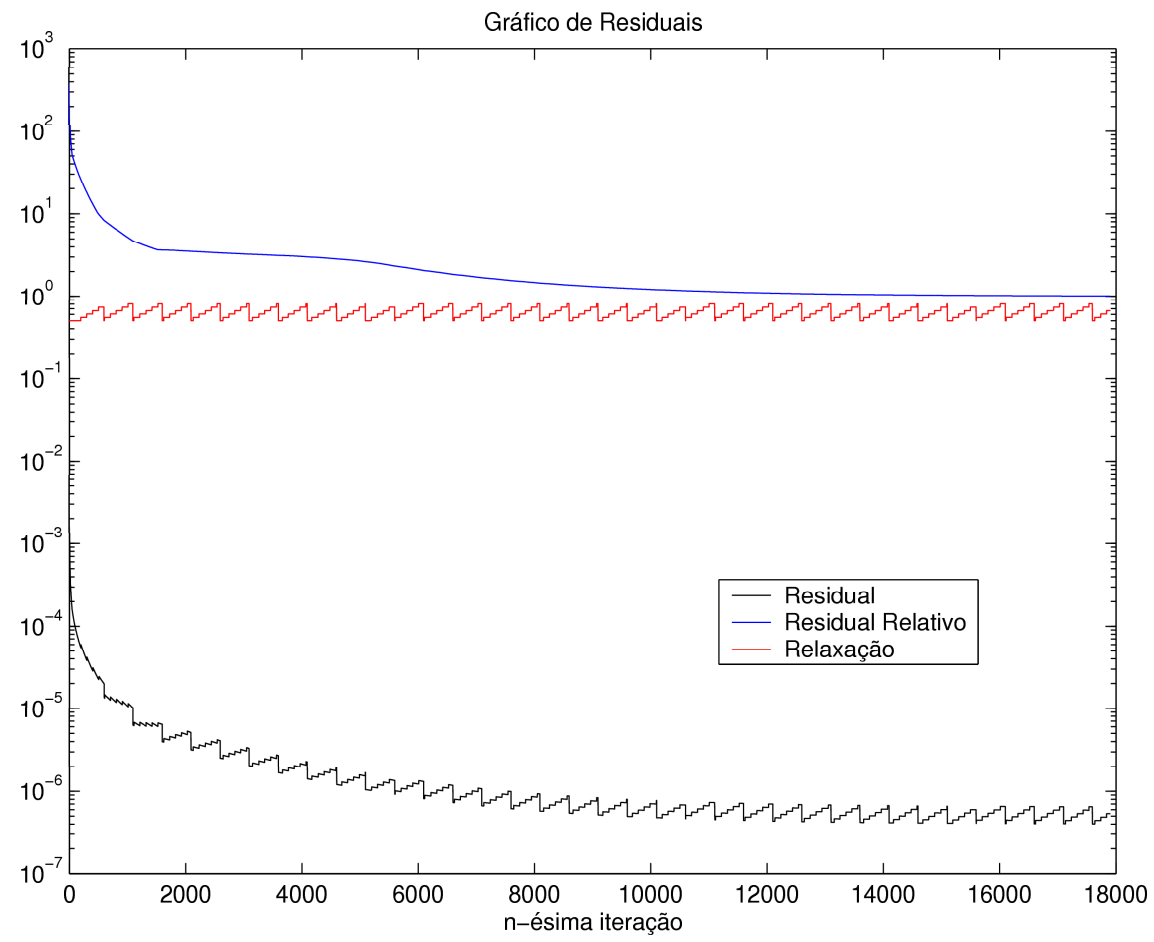

Figura A.6: Gráfico do residual usando TSD+Ent+Vort+CL para seção do perfil NACA0015 com $M_{\infty}=0$ e $\alpha=0,00^{\circ}$. 


\section{A.3 Resultados}

Pode-se observar nas Figuras A.7, A.8 e A.9 que a distribuição de pressão usando TSD+Ent+Vort e TSD+Ent+Vort+CL são praticamente iguais. Os casos analisados possuem espessura de deslocamento como mostram as Figuras A.10, A.11 e A.12. As curvas de distribuição de pressão simuladas possuem diferenças mínimas comparadas aos dados obtidos do Abbott ${ }^{[1]}$.

As Figuras A.13, A.14 e A.15 mostram o campo de velocidade, e nas Figuras A.16, A.17 e A.18 o potencial, todos em torno dos perfis NACA0012, NACA0006 e NACA0015, respectivamente. Pode-se observar nessas figuras a simetria em torno do perfil, mostrando a consistência das condições de contorno dadas na subseção 2.5.6. Além disso podemos observar a continuidade do fluxo potencial, sem salto, nas Figuras A.16, A.17 e A.18 devido a simetria do perfil e o ângulo de ataque ser nulo.

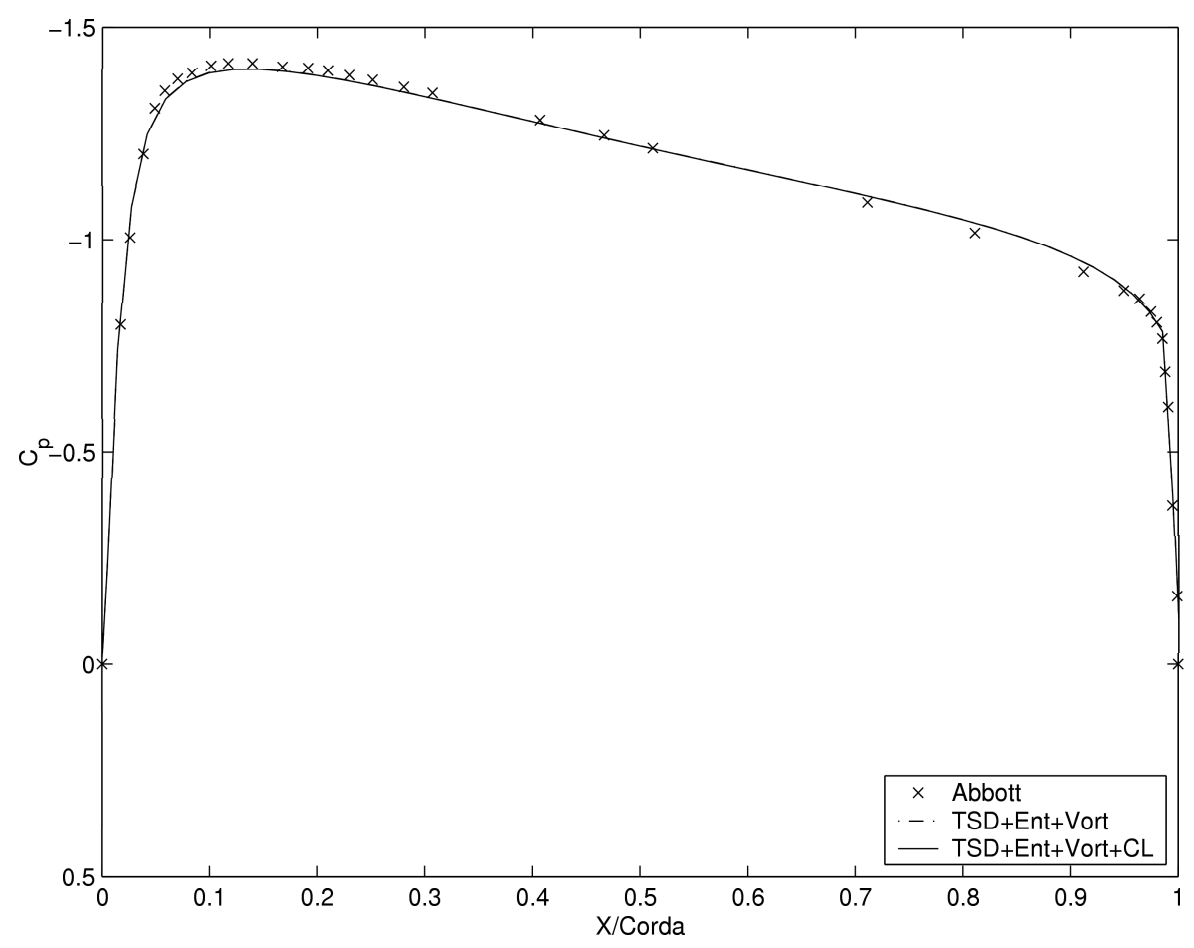

Figura A.7: Distribuição de $C_{p}$ para a seção NACA0012 $\operatorname{com} M_{\infty}=0$ e $\alpha=0^{\circ}$. 


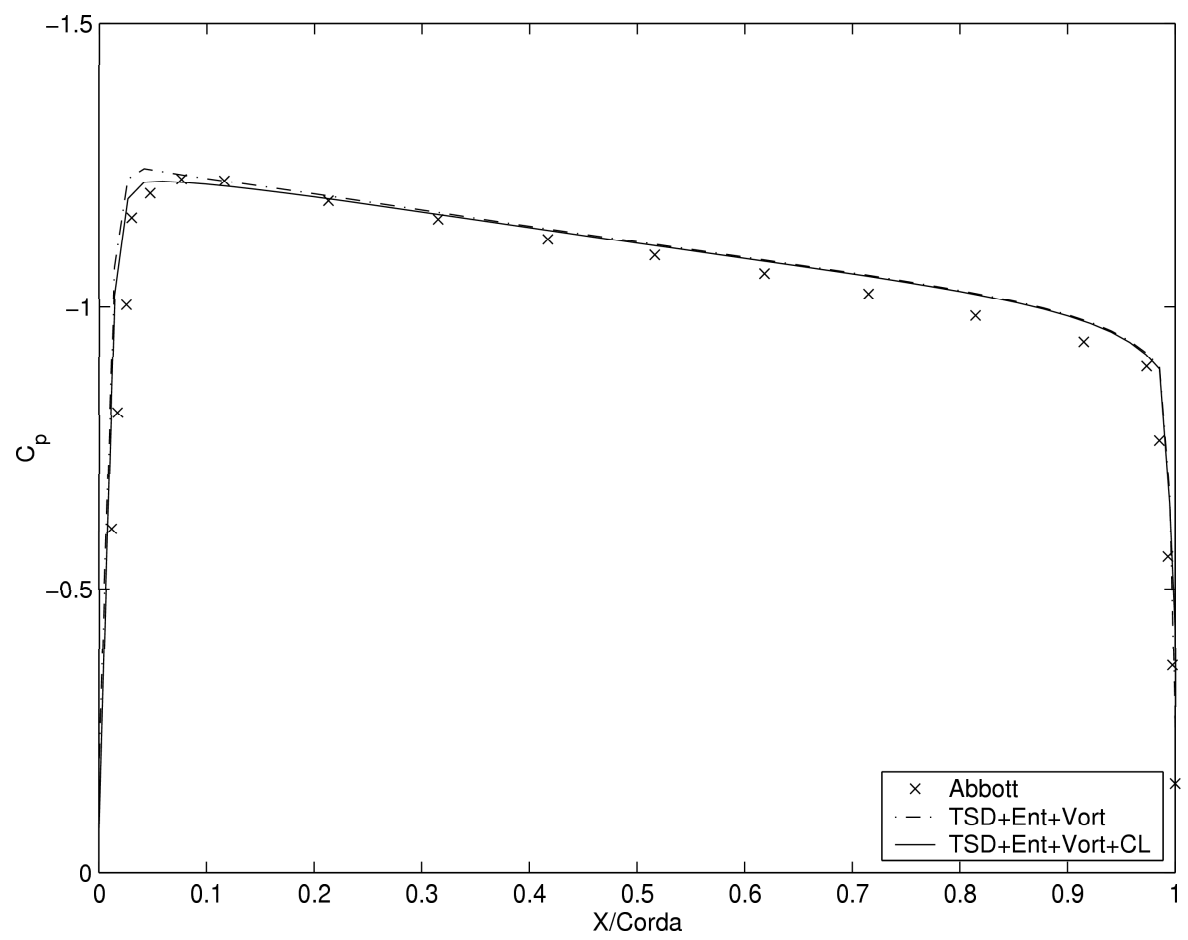

Figura A.8: Distribuição de $C_{p}$ para a seção NACA0006 com $M_{\infty}=0$ e $\alpha=0^{\circ}$.

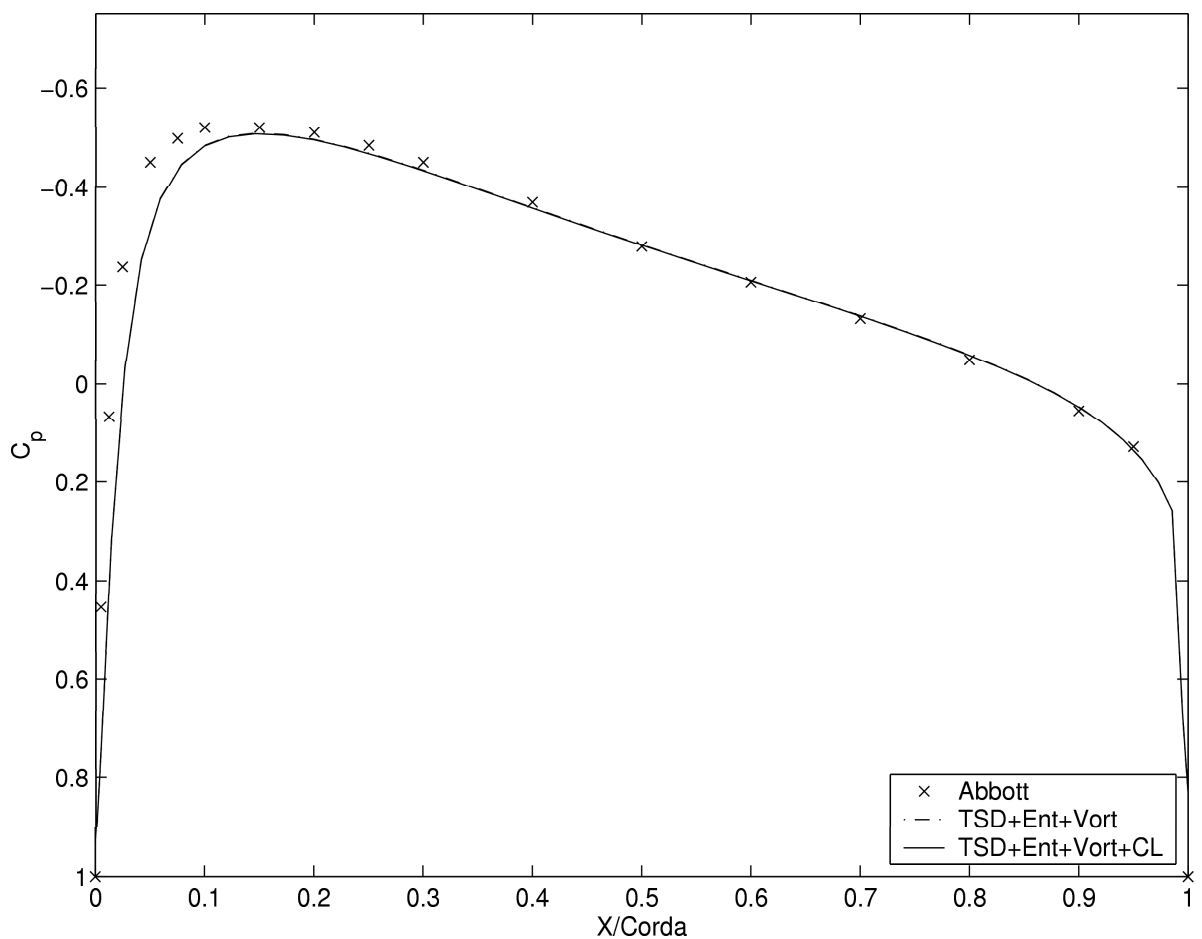

Figura A.9: Distribuição de $C_{p}$ para a seção NACA0015 com $M_{\infty}=0$ e $\alpha=0^{\circ}$. 


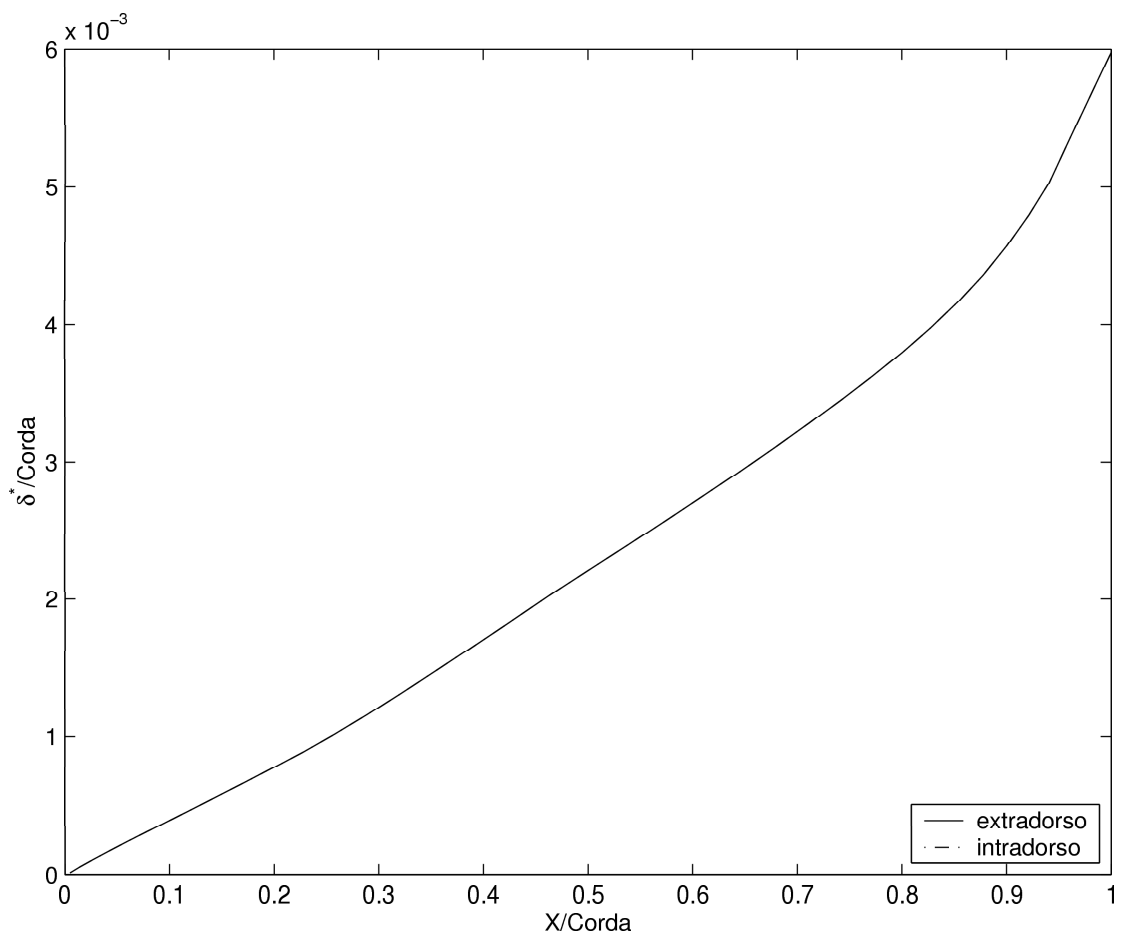

Figura A.10: Espessura de deslocamento para a seção NACA0012 com $M_{\infty}=0$ e $\alpha=0^{\circ}$.

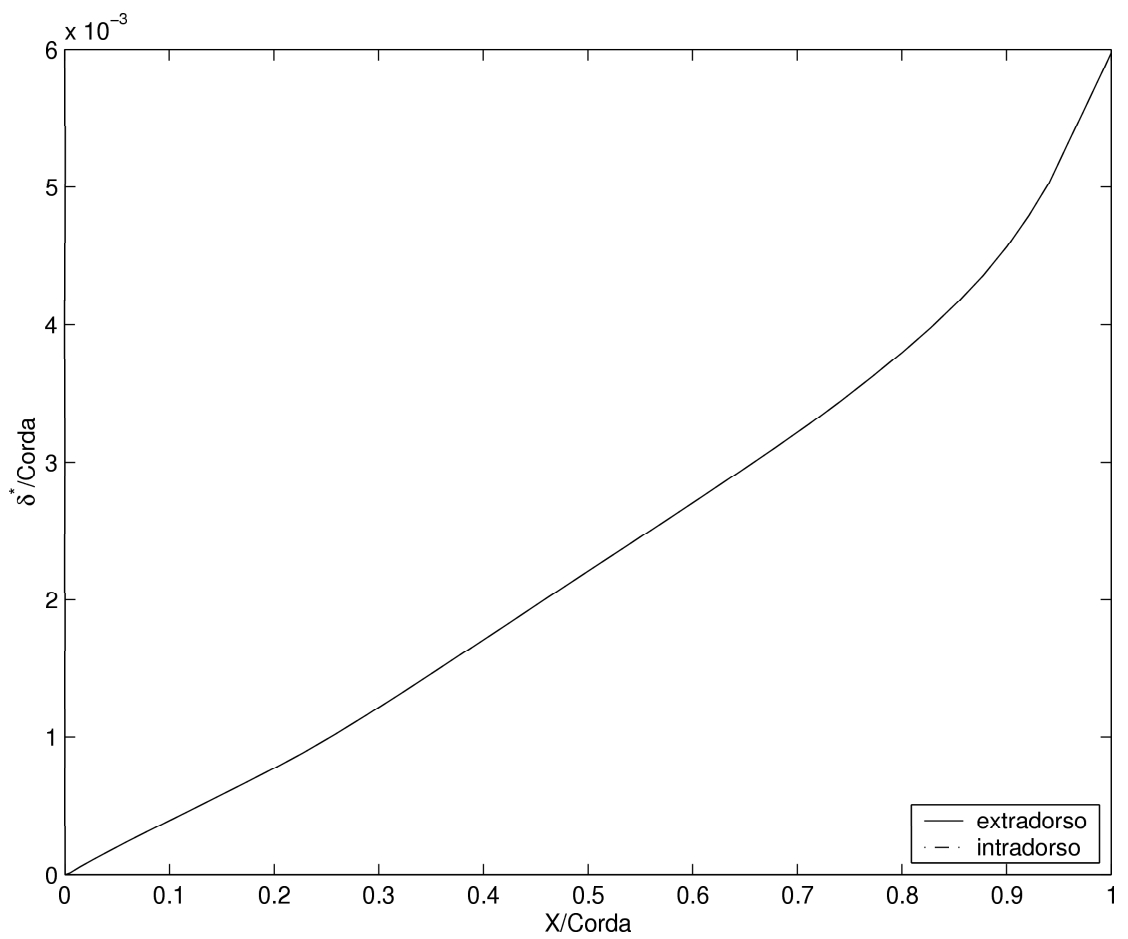

Figura A.11: Espessura de deslocamento para a seção NACA0006 com $M_{\infty}=0$ e $\alpha=0^{\circ}$. 


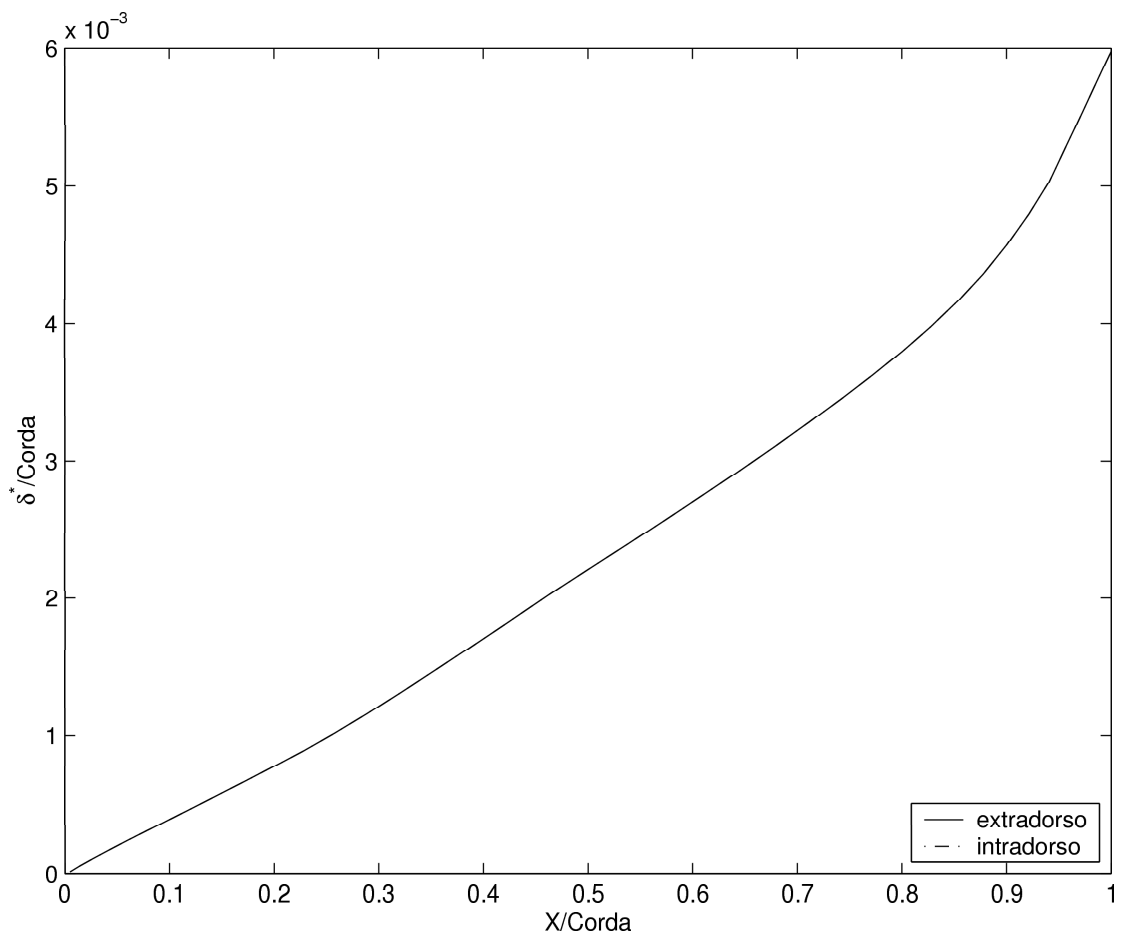

Figura A.12: Espessura de deslocamento para a seção NACA0015 com $M_{\infty}=0$ e $\alpha=0^{\circ}$.

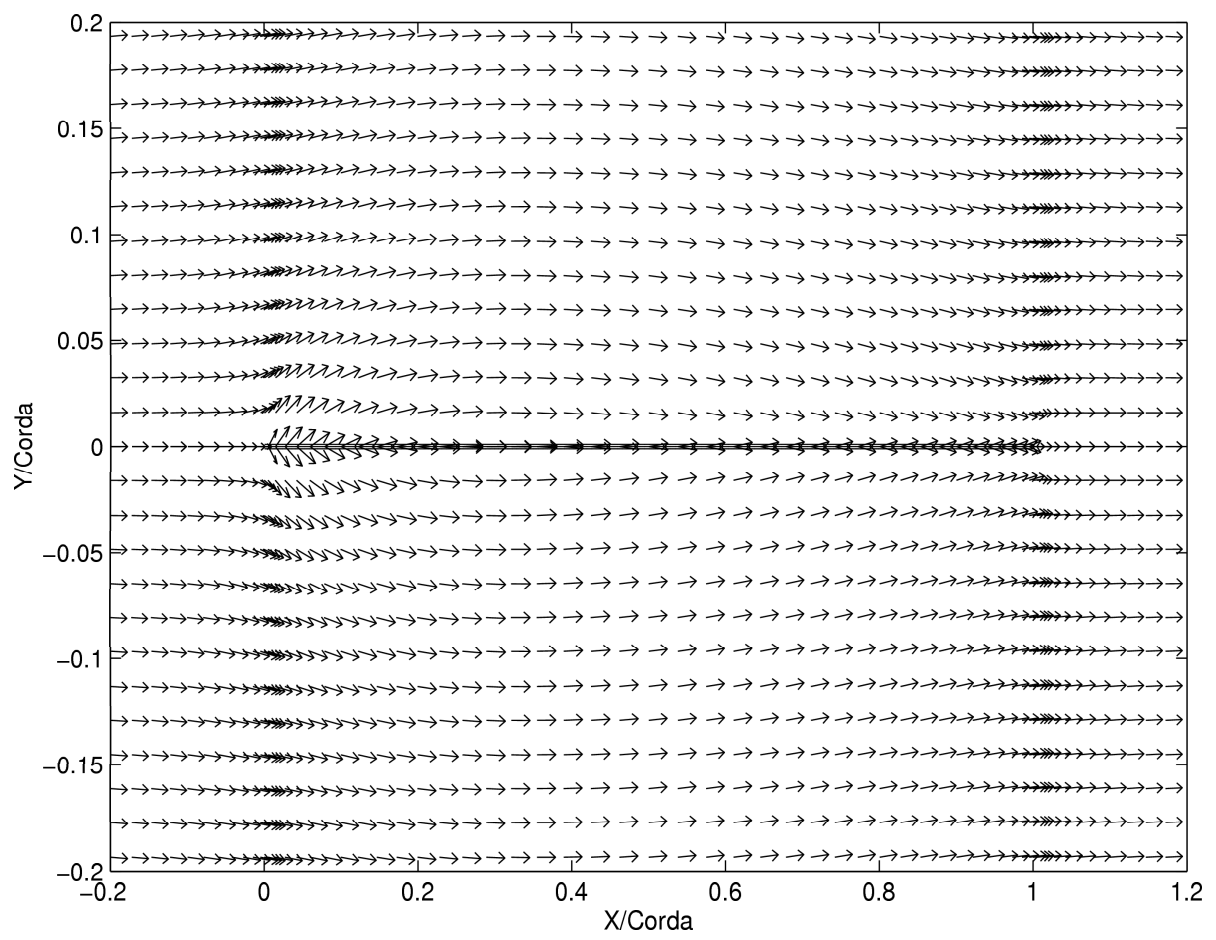

Figura A.13: Gráfico do campo de velocidade em torno do perfil NACA0012 com $M_{\infty}=0$ e $\alpha=0^{\circ}$, usando TSD+Ent+Vort+CL. 


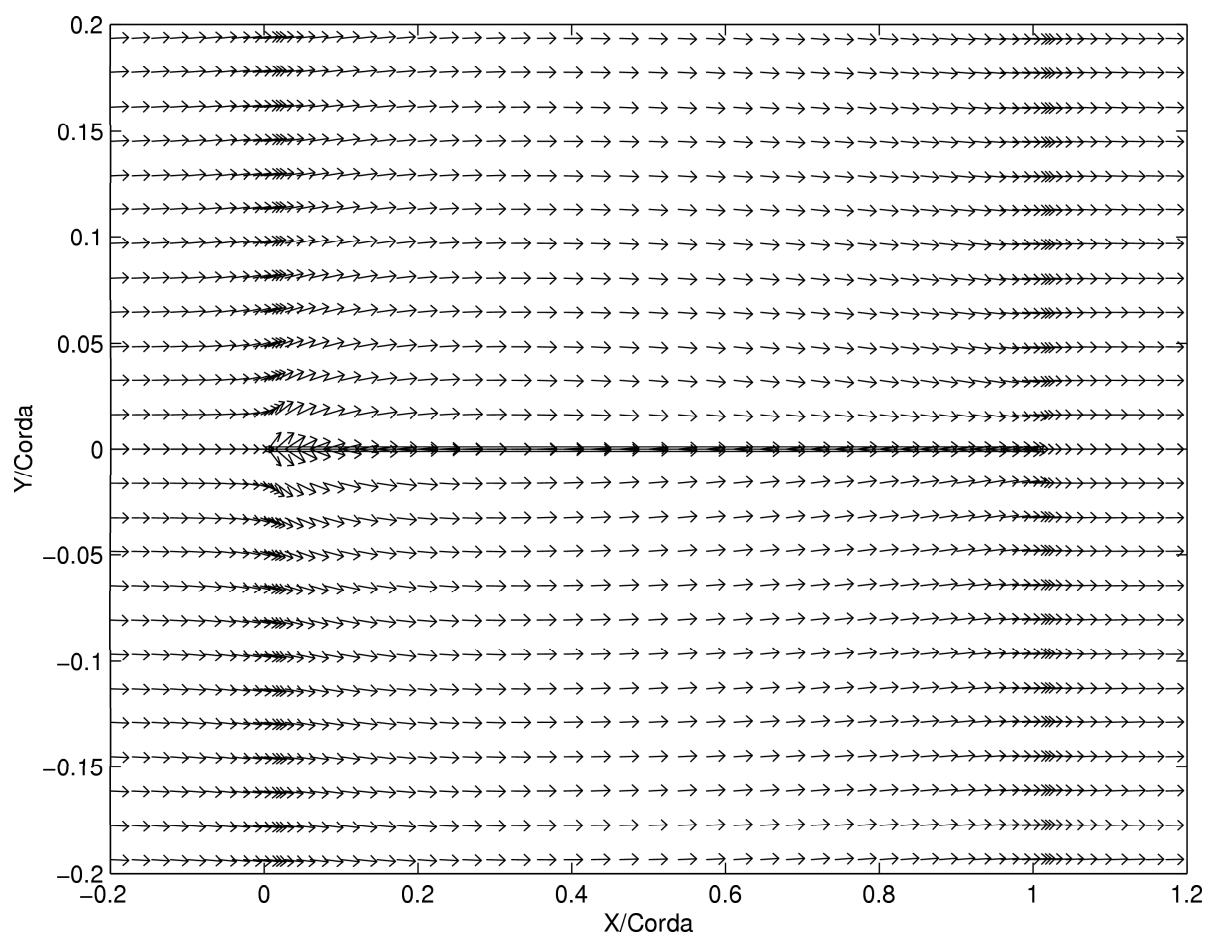

Figura A.14: Gráfico do campo de velocidade em torno do perfil NACA0006 $\operatorname{com} M_{\infty}=0$ e $\alpha=0^{\circ}$, usando TSD+Ent+Vort+CL.

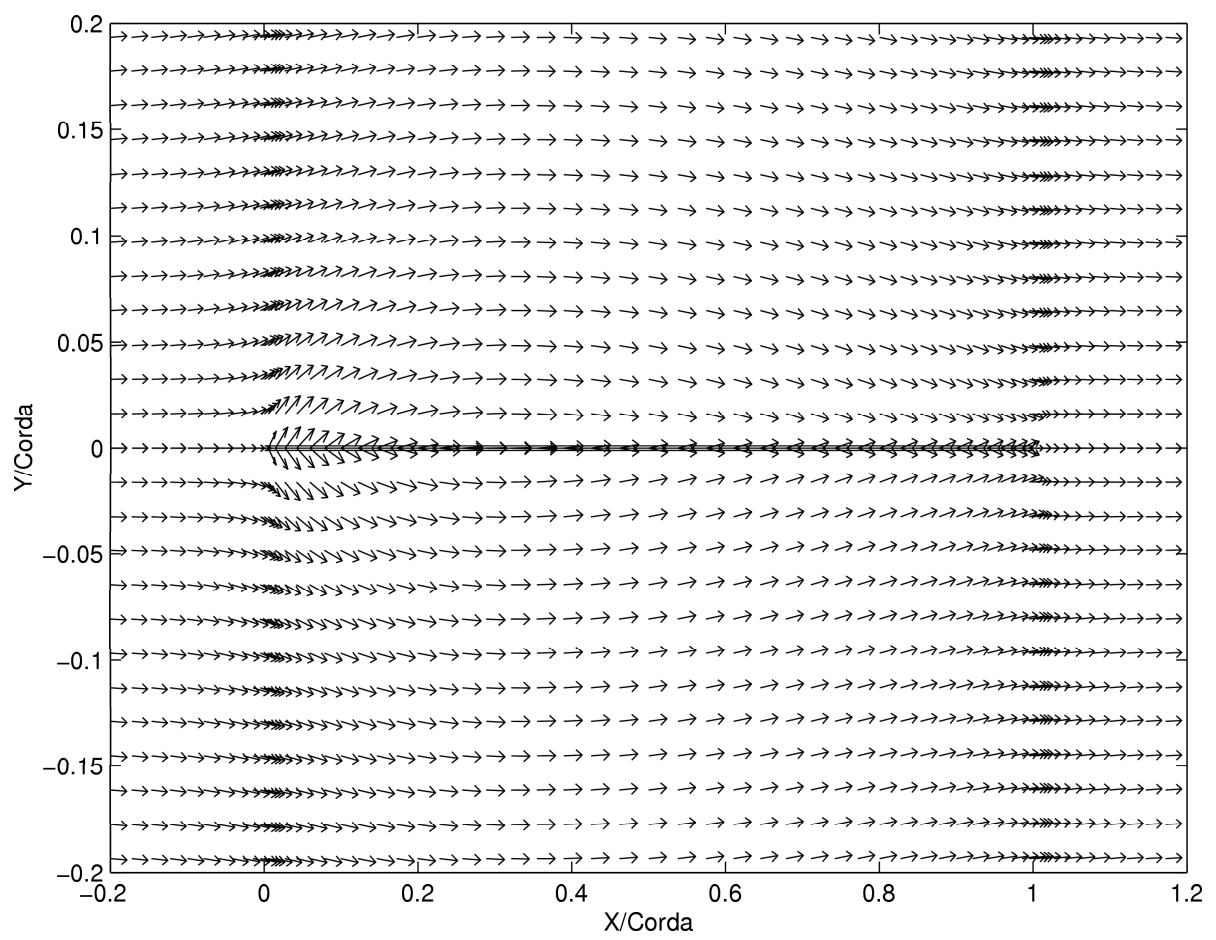

Figura A.15: Gráfico do campo de velocidade em torno do perfil NACA0015 $\operatorname{com} M_{\infty}=0 \mathrm{e}$ $\alpha=0^{\circ}$, usando TSD+Ent+Vort+CL. 


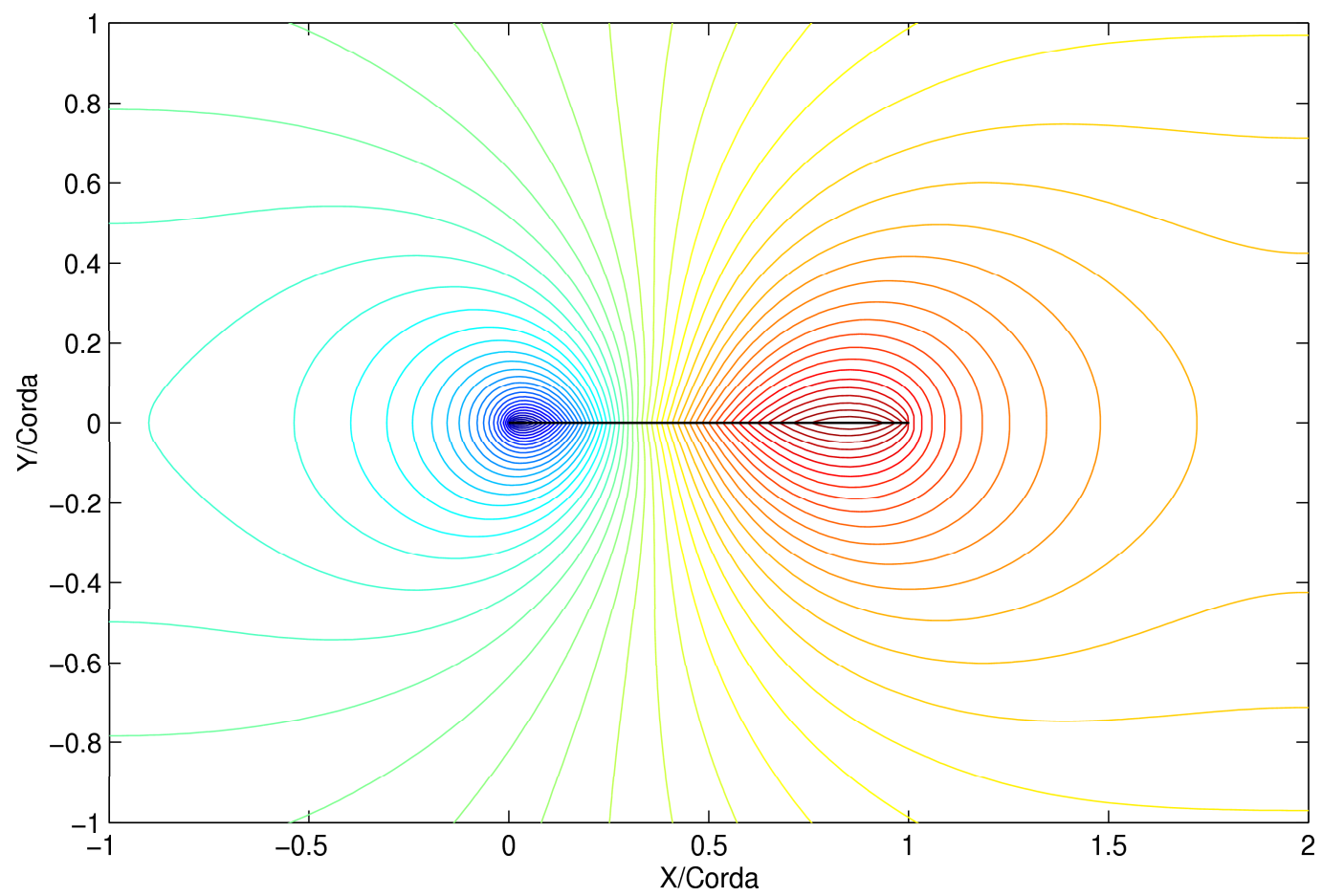

Figura A.16: Gráfico do potencial em torno do perfil NACA0012 com $M_{\infty}=0$ de entrada e $\alpha=0^{\circ}$, usando TSD+Ent+Vort+CL.

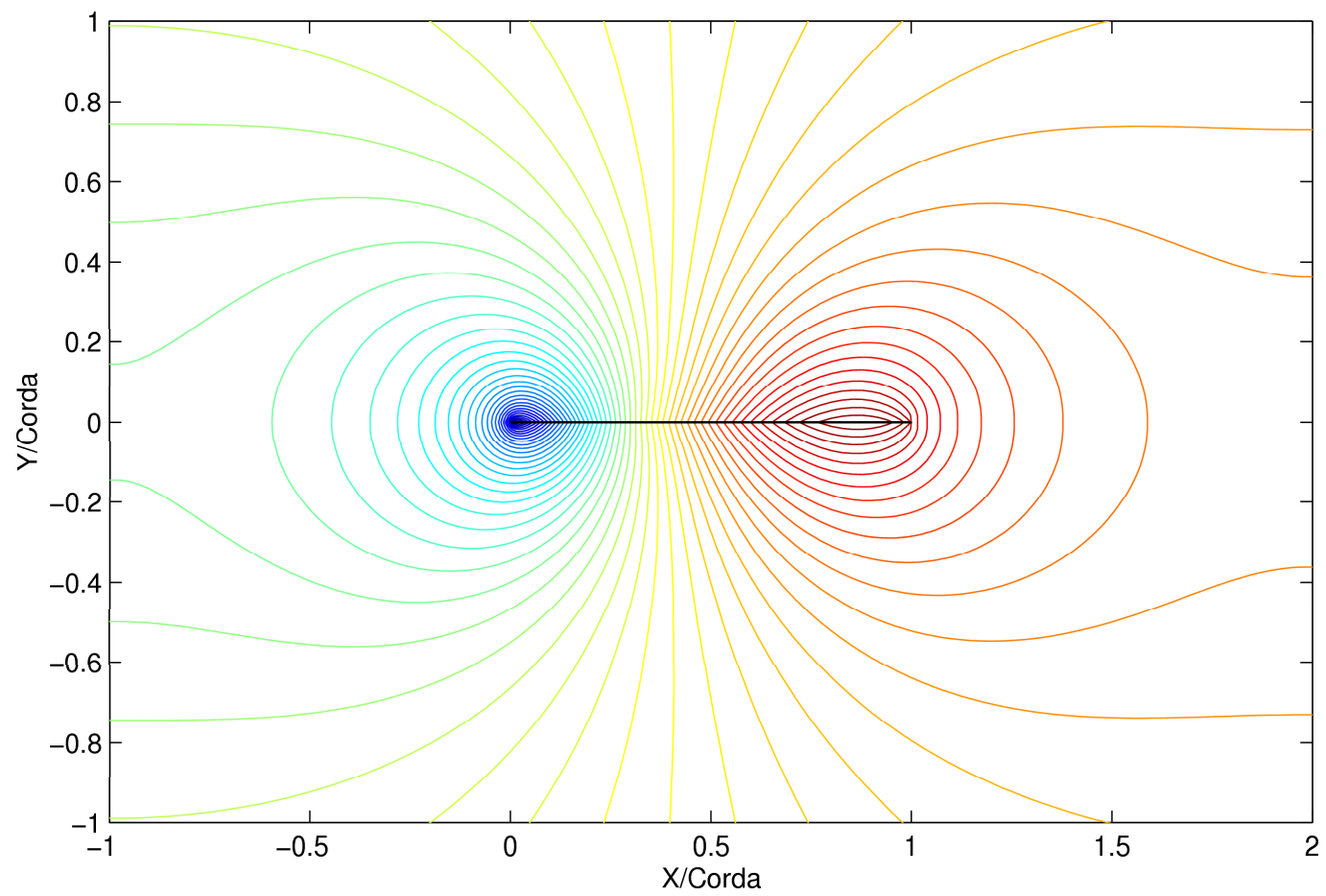

Figura A.17: Gráfico do potencial em torno do perfil NACA0006 com $M_{\infty}=0$ de entrada e $\alpha=0^{\circ}$, usando TSD+Ent+Vort+CL. 


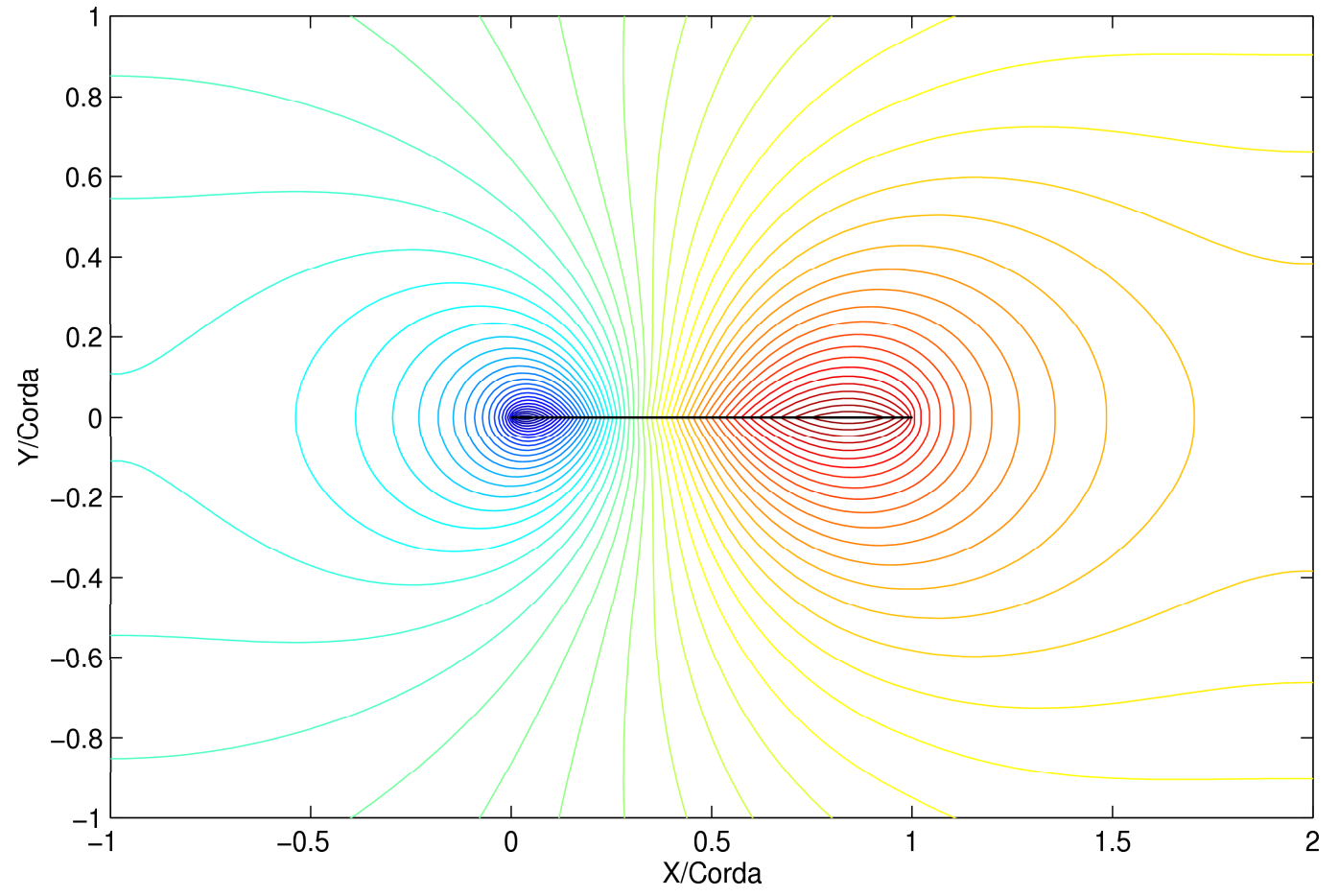

Figura A.18: Gráfico do potencial em torno do perfil NACA0015 com $M_{\infty}=0$ de entrada e $\alpha=0^{\circ}$, usando TSD+Ent+Vort+CL. 


\section{Referências Bibliográficas}

[1] ABBOTT, I.H.; DOENHOFF, A.E.V.(1959). Theory of Wing Sections - Including a Summary of Airfoil Data. New York: Dover Publications.

[2] ANDERSON, J.D.(1995). Computational Fluid Dynamics: the Basics with Applications. New York: McGraw-Hill Series in Mechanical Engineering.

[3] ANDERSON, D.A.; TANNEHILL, J.C.; FLETCHER, R.H. (1984). Computational Fluid Mechanics and Heat Transfer. New York: Hemisphere Publishing.

[4] BALLHAUS, W.F.; GOORJIAN, P.M.(1977). Implicit finite-difference computations of unsteady transonic flows about airfoils. AIAA Journal, v.15, p.1728-1735, Dec.

[5] BATINA, J.T. (1989). Unsteady small-disturbance theory including entropy and vorticity effects. Journal of Aircraft, v.26, n.6, p.531-538,June.

[6] BELK, D.M.; SIMPSON, L.B. (1989). Unsteady transonic flow using euler equations. NASA CP 3022, Part 1, p.215-241, Feb.

[7] BENDIKSEN, O.O.; KOUSEN, K.A. (1989). Transonic flutter calculations using the euler equations. NASA CP 3022, Part 2, p.477-491, Feb.

[8] BENNETT, R.; BATINA, J. (1989). Wing-flutter calculations with the CAP-TSD unsteady transonic small-disturbance program. Journal of Aircraft, Vol. 26, No. 9, pp 876-882.

[9] CHYU, W.J.; DAVIS, S.S.; CHANG, K.S. (1981). Calculation of unsteady transonic flow over an airfoil. AIAA Journal, v.19, p. 684-690, June.

[10] DOWELL, E.H. (1979). A Modern Course in Aeroelasticity. Alphen aan den Rijn: Sijthoff \& Noordhoff. 
[11] FORTUNA, A.O. Técnicas Computacionais para Dinâmica dos Fluidos : conceitos básicos e aplicações. São Paulo: EdUSP.

[12] FULSANG, D. F. (1985). Non-Isentropic Unsteady Transonic Small-Disturbance Theory. M. S. Thesis, Purdue Uni., West Lafayette, IN, May 1985.

[13] FUNG, K., SHIEH, T.H.(1993). Modeling, analysis, and prediction of flutter at transonic speeds. AIAA Journal, v.31, n.1, p. 140-147, Jan.

[14] GEURTS, E. G. M. (2000). F-5 Wing \& F-5 Wing + Tip Store.Verification and Validation Data for Computational Unsteady Aerodynamics, RESEARCH AND TECHNOLOGY ORGANIZATION, FRANCE, IN, October 2000.

[15] GOORJIAN, P.M. (1980). Implicit computations of unsteady transonic flow governed by the full potential equation in conservation form. In: AIAA AEROSPACE SCIENCES MEETING. 18., 1980. January, Pasadena, California. AIAA Paper 80-1050.

[16] GRECO JR, P.C.(1996). Transonic flutter and limit cycle oscillations. Ph.D Dissertation, University of Kansas.

[17] HAIDARI, A.H., MATTHEWS, B.(2003). Future trends for computational fluid dynamics in the process industry. In: INTERNATIONAL CONFERENCE ON CFD IN PROCESS INDUSTRIES. 3.,Melbourne, Australia. Australia.

[18] HOUGHTON, E.L.; CARRUTHERS, N.B.(1982) Aerodynamics for Engineering Students. 3.ed. London: Ed. Edward Arnold. p. 383-386.

[19] HOUWINK, R.; VAN DER VOOREN, J. (1980). Improved version of LTRAN2 for unsteady transonic flow computations. AIAA Journal, v.18, n.8, p.1008-1010.

[20] JORDAN, D.W.; SMITH, P. Nonlinear Ordinary Differential Equations. New York: Oxford University Press.

[21] KWAK, D. (1981). Non-Reflection far-field boundary conditions for unsteady transonic flow computation. AIAA Journal, v.19, p. 1401-1407, Nov. 
[22] LEE, C.S.(1990). A fast viscous correction method for transonic aerodynamics. In: MURTHY, T.K.S.; BREBBIA, C.A. Computational Methods in Viscous Aerodynamics. Amsterdam: Elsevier. Chapter 10, p. 301-330.

[23] Magnus, R.; Yoshihara, H. (1975). Unsteady Transonic Flow over an Airfoil. AIAA Journal, Vol. 13, December 1975, pp. 1622-1628.

[24] MALONE, J.B.; SANKAR, N.L. (1984). Numerical simulation of two-dimensional unsteady transonic flows using the Full-Potential Equation. AIAA Journal, v.22, n.8, p.1035-1041, Aug.

[25] MURMAN, E.M. (1974). Analysis of embedded shock waves calculated by relaxation methods. AIAA Journal, v.12, p.62-6, May.

[26] MURMAN, E.M.; COLE, J.D. (1971). Calculation of plane steady transonic flows. AIAA Journal, v.9, p. 114-121, Jan.

[27] NAYFEH, A.; MOOKk, D. (1979). Nonlinear Oscillations. John Wiley \& Sons.

[28] RIZZETTA, D.P.; BORLAND, C.J. (1983). Unsteady transonic flow over wings including viscous/inviscid interaction. AIAA Journal, v.21, p. 363-371, Mar.

[29] RIZZETTA, D.P.; CHIN, W.C. (1979). Effect of frequency in unsteady transonic flow. AIAA Journal, v.17, p. 779-781, July.

[30] SANSONE, G.; CONTI, R. (1964). Non-Linear differential equations. New York: Macmillan. p. 389-398.

[31] SASMAN, P. K.; CRESCI R. J. (1966). Compressible Turbulent Boundary Layer with Pressure Gradient and Heat Transfer. AIAA Journal, v.4, p. 19-25, Jan.

[32] SCHIPPERS, H.; HOUNJET, M.H.L. (1986). Calculation of 2-D unsteady transonic full potential flow about oscillating airfoils by two complementary approaches. In: AIAA APPLIED AERODYNAMIC CONFERECE., 4.,San Diego. Proceedings... California:AIAA.

[33] STEGER, J.L.; BAILEY, H.E. (1980). Calculation of transonic aileron buzz. AIAA Journal, v.18, p. 249-255, Mar. 
「34ך TANG, L.; BARTELS, R.E.; CHEN, P.C.; LIU, D.D. (2001). Simulation of transonic limit cycle oscillations using a CFD time-marching method. In: AIAA/ASME/AHS/ASC STRUCTURES, STRUCTURAL DYNAMICS AND MATERIALS CONFERENCE \& EXHIBIT, Seattle: AIAA/ASME/AHS/ASC, April AIAA 2001- 1290

[35] THIBERT,J.J.; OHMAN, L.L.(1979). NACA 0012 airfoil - pressure distributions, and boundary layer and wake measurements. (AGARD AR-138-Experimental data base for computer program assessment).

[36] UIUC Airfoil Coordinates Database - Version 2.0. Disponível em:

$<$ http://www.ae.uiuc.edu/m-selig/ads/coord database.html $>$.

Acesso em: 02 mar 2006.

[37] ZHANG, Z.; LIU, F. (2004). Calculations of unsteady flow and flutter by an euler and integral boundary-layer method on cartesian grids.In: APPLIED AERODYNAMICS CONFERENCE AND EXHIBIT, 22., Rhode Island, Proceedings... New York: AIAA. 